\title{
Interaction of the Epsin N-Terminal Homology domain (ENTH) with artificial membranes as a function of lateral tension
}

\author{
Dissertation \\ for the award of the degree \\ Doctor rerum naturalium \\ within the doctoral program \\ Physics of Biological and Complex Systems \\ of the Georg-August-University of Science (GAUSS)
}

\author{
submitted by \\ Martin Gleisner \\ from Weimar
}

Göttingen 2016 



\title{
Interaction of the Epsin N-Terminal Homology domain (ENTH) with artificial membranes as a function of lateral tension
}

\author{
Dissertation \\ for the award of the degree \\ Doctor rerum naturalium \\ within the doctoral program \\ Physics of Biological and Complex Systems \\ of the Georg-August-University of Science (GAUSS)
}

\author{
submitted by \\ Martin Gleisner \\ from Weimar
}

Göttingen 2016 
Members of the thesis committee

Prof. Dr. Claudia Steinem,

Institut für Organische und Biomolekulare Chemie,

Georg-August-Universität Göttingen

Prof. Dr. Jörg Enderlein,

Drittes Physikalisches Institut,

Georg-August-Universität Göttingen

Prof. Dr. Sarah Köster,

Institut für Röntgenphysik,

Georg-August-Universität Göttingen

Members of the examination board

Referee: Prof. Dr. Claudia Steinem,

Institut für Organische und Biomolekulare Chemie,

Georg-August-Universität Göttingen

$2^{\text {nd }}$ referee: Prof. Dr. Michael Meinecke,

European Neuroscience Institute,

Georg-August-Universität Göttingen

Further members of the examination board

Prof. Dr. Sarah Köster,

Institut für Röntgenphysik,

Georg-August-Universität Göttingen

Prof. Dr. Jörg Enderlein,

Drittes Physikalisches Institut,

Georg-August-Universität Göttingen

Prof. Dr. Burkhard Geil,

Institut für Physikalische Chemie,

Georg-August-Universität Göttingen

Dr. Sebastian Kruss,

Institut für Physikalische Chemie,

Georg-August-Universität Göttingen

Date of oral examination

$18^{\text {th }}$ July 2016 


\section{Declaration}

I, Martin Gleisner, hereby certify that my doctoral thesis entitled "Interaction of the Epsin N-Terminal Homology domain (ENTH) with artificial membranes as a function of lateral tension" has been written independently and with no other sources and aids than quoted.

Göttingen, 2016

Martin Gleisner 
Inmitten der Schwierigkeiten liegt die Möglichkeit.

Albert Einstein 


\section{Contents}

1 Introduction 1

1.1 Generation of membrane curvature in biological membranes . . . . 1

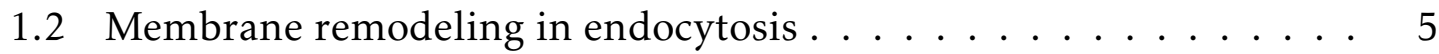

1.3 Membrane tension as a regulator in endocytosis . . . . . . . . . 7

1.4 Epsin N-terminal homology domain . . . . . . . . . . . . 9

$\begin{array}{lll}2 & \text { Scope of thesis } & 13\end{array}$

3 Materials and methods 15

3.1 Osmotic pressure . . . . . . . . . . . . . . . . 15

$\begin{array}{lll}3.1 .1 & \text { Determination of the osmolality by freezing point depression } 17\end{array}$

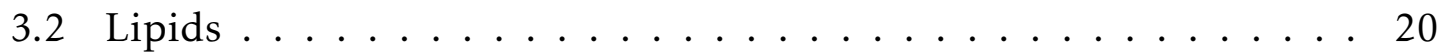

3.3 Proteins . . . . . . . . . . . . . . . . . . . . 26

$3.3 .1 \quad$ Epsin N-terminal homology domain . . . . . . . . . . . 26

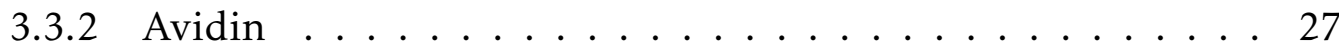

3.4 Fluorescence microscopy . . . . . . . . . . . . . . . . . 28

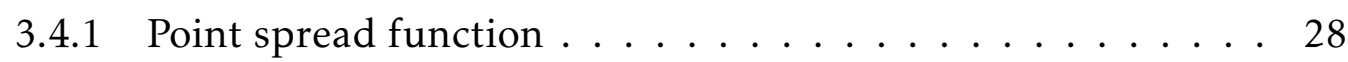

3.4 .2 Digitalization of an analog signal . . . . . . . . . . . 30

3.4 .3 Confocal laser scanning microscopy (CLSM) . . . . . . . . . 31

3.4 .4 Spinning disc confocal laser microscopy (SDCLM) . . . . . . 32

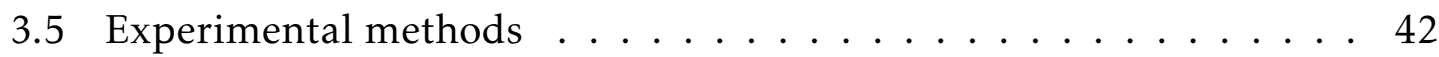

3.5 .1 Preparation of unilamellar vesicles . . . . . . . . . . 42

3.5 .2 Preparation of pore-spanning membranes . . . . . . . . . 43

3.5.3 Immobilization of biotinylated giant unilamellar vesicles onto an avidin coated glass slide $\ldots \ldots \ldots$. . . . . . 44

3.6 Biosensing techniques . . . . . . . . . . . . . . . . 44

3.6 .1 Surface plasmon resonance spectroscopy . . . . . . . . . . . 45

3.6 .2 Reflectometric interference spectroscopy. . . . . . . . . . . 48 
3.7 Scanning probe microscopy $\ldots \ldots \ldots \ldots \ldots \ldots \ldots \ldots$

3.7 .1 Atomic force microscopy . . . . . . . . . . . . . . . 51

3.7 .2 Scanning ion conductance microscopy . . . . . . . . . 55

3.7 .3 Scanning electron microscopy _ . . . . . . . . . . . . 58

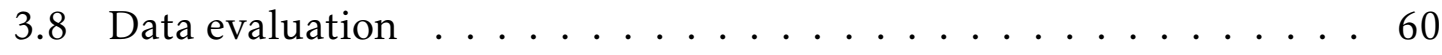

3.8 .1 Determination of the GUV geometry . . . . . . . . . . 60

3.8 .2 Influence of electrostatics and osmotic gradients on the GUV

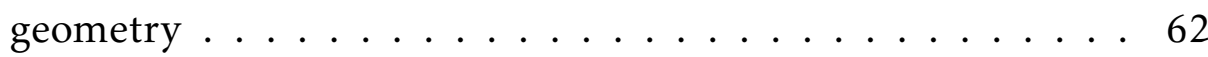

4 Results 65

4.1 Determination of the dissociation constant of ENTH binding to PIP 265

4.1 .1 Reflectometric interference spectroscopy . . . . . . . . . . 65

4.1 .2 Surface plasmon resonance spectroscopy . . . . . . . . . . 68

$4.1 .3 \quad$ Average dissociation constant of ENTH binding to PIP 2 . . . 70

4.2 Generation and characterization of nanometer-sized protruded porespanning membranes . . . . . . . . . . . . . . 71

$4.3 \quad$ Interaction of ENTH with nanometer-sized protruded pore-spanning

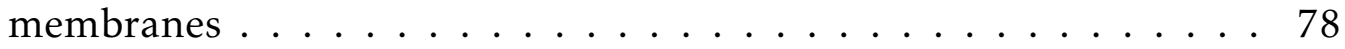

4.4 Generation and characterization of micrometer-sized protruded pore-spanning membranes . . . . . . . . . . . . . 83

4.4.1 Influence of the substrate dimension on the determination of the lateral membrane tension . . . . . . . . . . . . . . . 83

4.4 .2 Height and lateral tension of micrometer-sized protruded pore-spanning membranes . . . . . . . . . . . . 86

4.4.3 Lateral membrane tension of micrometer-sized protruded pore-spanning membranes measured by atomic force micros-

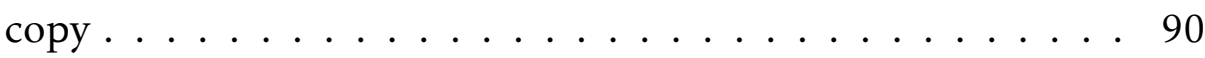

4.5 Adhered GUVs as a model system to analyze tension dependent

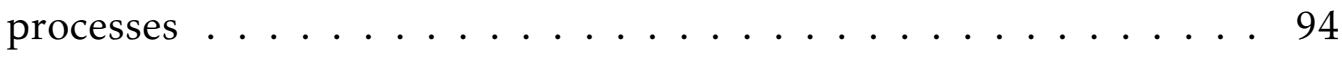

4.5 .1 Theoretical model . . . . . . . . . . . . . . . . . . . . . 94

4.5.2 Adhesion of biotinylated GUVs adhering to an avidin-coated glass surface as a function of the $\mathrm{Mg}^{2+}$ concentration. . . . . 100

4.5.3 Adhesion energy and lateral membrane tension of GUVs adhering to an avidin-coated glass surface . . . . . . . . 102 
4.6 Quantification of fluorophore surface densities . . . . . . . . 105

4.6 .1 Distribution of $\mathrm{PIP}_{2}$ in GUVs $\ldots \ldots \ldots \ldots$

4.6 .2 Intensity calibration . . . . . . . . . . . . . . 107

4.6 .3 Binding of ENTH-Atto594 to giant unilamellar vesicles . . . 111

4.7 Interaction of ENTH with adhered GUVs as a function of the lateral membrane tension . . . . . . . . . . . . . . . . . . . 113

$4.7 .1 \quad$ ENTH binding to adhered GUVs . . . . . . . . . . . . 113

4.7.2 Quantification of the change in area as a result of ENTH

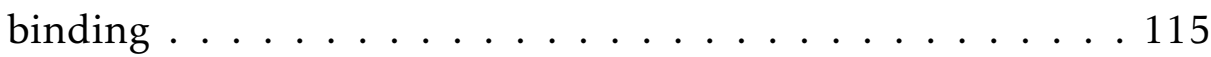

$\begin{array}{lll}5 \text { Discussion } & 121\end{array}$

$5.1 \quad$ Binding affinity of ENTH $\ldots \ldots \ldots \ldots \ldots \ldots$

5.2 Protruded pore-spanning membranes. . . . . . . . . . . 126

5.2 .1 Curvature of protruded pore-spanning membranes. . . . . 126

5.2.2 Lateral membrane tension of protruded pore-spanning membranes . . . . . . . . . . . . . . 128

5.2.3 Interaction of ENTH with protruded pore-spanning membranes . . . . . . . . . . . . . . . 134

5.3 Lateral membrane tension of GUVs adhering to an avidin-coated glass surface $\ldots \ldots \ldots \ldots \ldots . \ldots \ldots 137$

5.3 .1 Adhesion of GUVs to a surface $\ldots \ldots \ldots$. . . . . . 137

5.3.2 Adhesion energy and lateral membrane tension of adhered

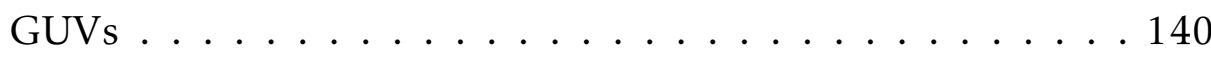

5.4 Binding of ENTH to GUVs $\ldots \ldots \ldots \ldots \ldots$

5.4 .1 Binding of ENTH-Atto594 to GUVs . . . . . . . . . . . . 142

5.4 .2 Quantification of the changes in membrane area caused by ENTH binding . . . . . . . . . . . . . . . . . . . 144

5.4 .3 Binding of ENTH to GUVs with a low membrane tension . . 147

5.4.4 Theoretical predictions for membrane curvature inducing

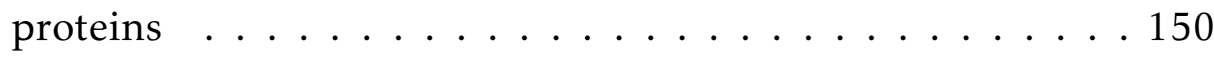

5.4 .5 Binding of ENTH to GUVs with high membrane tension. . . 152

$\begin{array}{ll}6 \text { Conclusion } & 155\end{array}$

\begin{tabular}{lll}
\hline 7 & Bibliography & 157
\end{tabular} 
\begin{tabular}{ll}
\hline A Appendix & 181
\end{tabular}

A.1 List of Figures . . . . . . . . . . . . . . . . . . . 181

A.2 List of Schemes . . . . . . . . . . . . . . . . . . . . 185

A.3 List of Tables . . . . . . . . . . . . . . . . . . . . . . . . 185

A.4 Coworkers . . . . . . . . . . . . . . . . . . 186

A.5 Sequence of ENTH . . . . . . . . . . . . . . . . . 186

A.6 Abbreviations . . . . . . . . . . . . . . . . . . 186

A.7 Chemicals and consumables . . . . . . . . . . . . . . . . . 189

A.8 Devices . . . . . . . . . . . . . . . . . . . . . . . . . . 191

A.9 Software . . . . . . . . . . . . . . . . . . . . 193 
Aвstract. During endocytosis, membrane remodeling of a planar membrane into a highly curved vesicle is controlled by a complex protein machinery. However, the regulatory role of physical properties such as membrane tension is highly debated. At the beginning of the formation of a vesicle during clathrin mediated endocytosis, the protein epsin binds to its receptor lipid phosphatidylinositol-4,5-bisphosphate $\left(\mathrm{PIP}_{2}\right)$. Upon binding, a previously unstructured part of the epsin $\mathrm{N}$-terminal homology domain (ENTH) forms an $\alpha$-helix, which inserts into the membrane.

Experiments using artificial lipid bilayers were performed to study the interaction of ENTH and lipid bilayers as a function of lipid composition and membrane tension. The binding affinity of ENTH to $\mathrm{PIP}_{2}$ was analyzed for different lipid compositions and membrane topologies. Similar affinities of ENTH binding to $\mathrm{PIP}_{2}$ in the high nanomolar range were measured, independent of the used lipid composition and membrane topology investigated.

Protruded pore-spanning membranes were established to investigate the remodeling activity of ENTH as a function of lipid composition. Binding of ENTH to membranes having a large area compressibility modulus and a high lysis tension resulted in growth of the membrane protrusions. Binding of ENTH to membranes with a lower area compressibility modulus and lysis tension resulted in the formation of membrane defects. For all lipid compositions analyzed, no vesiculation or tubulation was observed after binding of ENTH.

To analyze whether the high membrane tension of the protruded pore-spanning membranes suppresses any membrane remodeling ability of ENTH, experiments with giant unilamellar vesicles adhering to a solid support were conducted. The vesicles' adhesion strength and lateral tension was adjusted to analyze the ENTH's remodeling ability at lateral tensions corresponding to low and high membrane tensions found in cells. The formation of membrane tubes was observed for vesicles having a low membrane tension. Increasing the membrane tension resulted in a suppression of tube formation.

Independent of the membrane tension, flattening of the vesicles was monitored after binding of ENTH. The inserted ENTH helix disturbs lipid packing, which reduces the area compressibility modulus and thus the bending rigidity of the membranes. The reduced bending rigidity lowers the energy required for the generation of membrane curvature. As the inserted helix of ENTH also splays the lipid head groups, thereby inducing local curvature, the combination of both mechanisms is expected to efficiently initiate the formation of a vesicle during clathrin mediated endocytosis. 
Zusammenfassung. Proteine formen während der Endozytose planare Membranen zu gekrümmten Vesikeln um. Im ersten Schritt dieses Prozesses bindet das Protein Epsin an das Rezeptorlipid Phosphatidylinositol-4,5-bisphosphat ( $\left.\mathrm{PIP}_{2}\right)$ und ein vorher ungeordneter Bereich am N-Terminus von Epsin, die epsin N-terminal homology domain (ENTH), bildet eine $\alpha$-Helix, welche in die Membran insertiert. Im Rahmen dieser Arbeit wurde die Wechselwirkung von ENTH mit $\mathrm{PIP}_{2}$-haltigen Lipiddoppelschichten unter Verwendung von bottom up Modellsystemen charakterisiert. Die Affinität von ENTH zu $\mathrm{PIP}_{2}$ wurde für verschiedene Lipidzusammensetzungen und Membrangeometrien untersucht, wobei unabhängig von Lipidzusammensetzung und Membrantopologie ähnliche Bindungskonstanten im hohen nanomolaren Bereich bestimmt wurden.

Ausgestülpte porenüberspannende Membranen wurden als Modellsystem etabliert, um die Fähigkeit von ENTH zur Membrankrümmung als Funktion der Lipidzusammensetzung zu charakterisieren. Die Höhe der ausgestülpten Membranen ist durch die laterale Spannung begrenzt. Verursacht durch Insertion der ENTH Helix wuchsen Membranen mit einem hohen Flächenkompressionsmodul. Im Gegensatz dazu rissen Membranen mit einem niedrigen Flächenkompressionsmodul durch die ENTH induzierte Bildung von Membrandefekten.

Entgegen der Eigenschaft von ENTH Membranen zu krümmen, wurde an hochgespannten porenüberspannenden Membranen keine Membrantubulierung und -vesikulierung beobachtet. Daher wurde untersucht, ob diese Fähigkeit durch eine hohe laterale Spannung unterdrückt wird. Zu diesem Zweck wurden Riesenvesikel auf einem Glassubstrat adhäriert, wobei die Adhäsionsstärke und in Folge die laterale Spannung als Funktion der $\mathrm{Mg}^{2+}$ Konzentration eingestellt werden konnte. ENTH-induzierte Membrantubulierung konnte für Vesikel mit niedriger Spannung nachgewiesen werden und war bei höherer Spannung unterdrückt.

Unabhängig von der Membranspannung wurde ein Abflachen der Vesikel nach ENTH-Zugabe beobachtet. Die Ursache hierfür wurde in der durch die insertierte Helix hervorgerufene Reduktion des Flächenkompressionsmoduls gefunden. Die insertierte Helix stört die hydrophoben Wechselwirkungen der Lipidfettsäureketten und das reduzierte Flächenkompressionsmodul verringert die zur Membrankrümmung benötigte Energie. In Kombination mit der durch die insertierte Helix erzeugten lokalen Krümmung ist dies eine molekulare Erklärung für die ENTH-initiierte Bildung eines Vesikels während der Endozytose. 


\section{Introduction}

Lipid bilayers forming cell membranes are barriers separating the cell interior from the environment. Cells have developed mechanisms to overcome this natural barrier to take up nutrition and other compounds. One pathway is endocytosis which can be divided into phagocytosis and pinocytosis. [1] During phagocytosis solid matter larger than $0.5 \mu \mathrm{m}$ in diameter is internalized. Pinocytosis describes the uptake of fluids and particles and can be divided into macro- and micropinocytosis. During macropinocytosis the cell membrane forms a vesicle ranging from $0.2 \mu \mathrm{m}$ to $10 \mu \mathrm{m}$ in diameter enclosing particles and fluids within the vesicle. Micropinocytosis is similar to macropinocytosis, but the formed vesicles are smaller than $0.2 \mu \mathrm{m}$. As the formation of a vesicle from a planar membrane is a highly complex process, it is regulated by a complex protein machinery to ensure a precise timing and exact spatial precision. During formation and fission of a vesicle, regions with high curvature are generated, which require mechanisms mediating the necessary changes in membrane geometry.

\subsection{Generation of membrane curvature in biological membranes}

Cell membranes constantly change their shape during processes such as cell movement, cell division and vesicle trafficking, thereby forming regions of different curvature. During endocytosis, the planar membrane forms a bud, which then evolves into a highly curved vesicle. A precise regulation of the changes in shape is required to minimize the energy for the formation of these curved structures. The release of the bending energy resulting from the highly curved region at the vesicle neck is a main driving force of membrane fission. [2] 
It can be distinguished between positive and negative curvature whereby regions curved towards the cell interior are described as positive curvature. Therefore, membrane buds as formed during early stages of endocytosis are mainly positively curved (Figure 1.1A). Later, when an endocytotic vesicle has formed, the transition region between vesicle and neck is characterized by high positive and negative curvature. The neck itself has a cylindrical shape with zero curvature along the longitudinal axis and positive curvature perpendicular to this axis (Figure 1.1 B). [3]

A

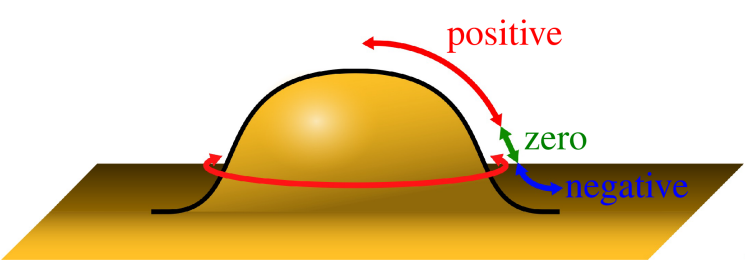

B

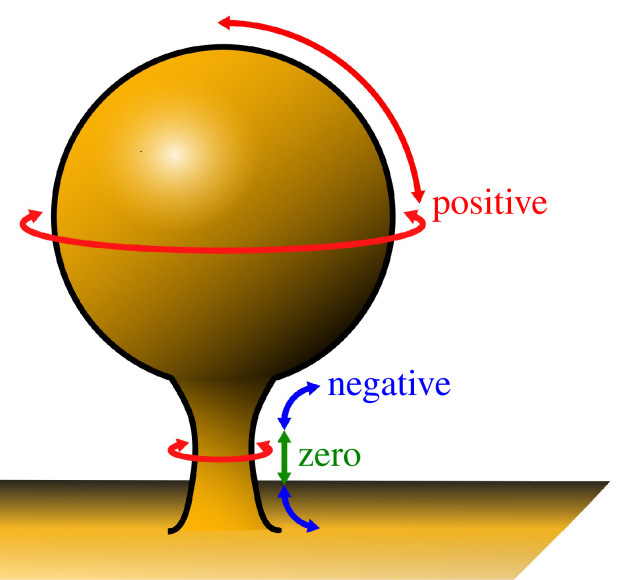

Figure 1.1: Schematic illustration of the different types of curvature of a membrane bud (A) and a vesicle (B). Red: positive curvature, green: no curvature, blue: negative curvature. Derived from McMahon et al. ${ }^{3}$

Generally, two different pathways are conceivable for the generation of membrane curvature. It can be induced either by changes in lipid composition and asymmetry or by proteins. Curvature is accepted not only as a result of processes shaping lipid bilayers but believed to actively regulate those processes.

Lipids have a preferred curvature (spontaneous curvature) which depends on the lipid shape. Clustering of lipids with a curvature promoting shape in one monolayer leads to bending of the entire membrane due to the intermonolayer coupling (Figure 1.2A). ${ }^{[4}$ Lipids are also receptors for many proteins, which allows controlling density of curvature inducing proteins by regulating the concentration of receptor lipids in the lipid bilayers and has been shown in vivo to either promote or inhibit the formation of protein induced structures with high curvature. [5] Membrane bending by proteins can be induced by the generation of an asymmetry between the lipid monolayers, by e.g. proteins scaffolding cell membranes, 
the insertion of hyrophobic motifs into the membrane, protein oligomerization and membrane-cytoskeleton interactions. [3] Canonically shaped transmembrane proteins can mold membranes to their preferred curvature which is determined by the protein's structure (Figure 1.2 B). Clustering of proteins enlarges the induced curvature as observed for many transmembrane receptors and channels. [6, 7] Clustering can induce membrane curvature even if the proteins itself does not have a canonical shape. Conformational changes of the clustered protein, e.g. as a response to binding of ligands, can then lead to membrane bending. Also, a very high density of proteins at the lipid bilayer is discussed to be sufficient to drive membrane curvature even though the individual proteins do not have curvature inducing properties (protein crowding). [8, 9]

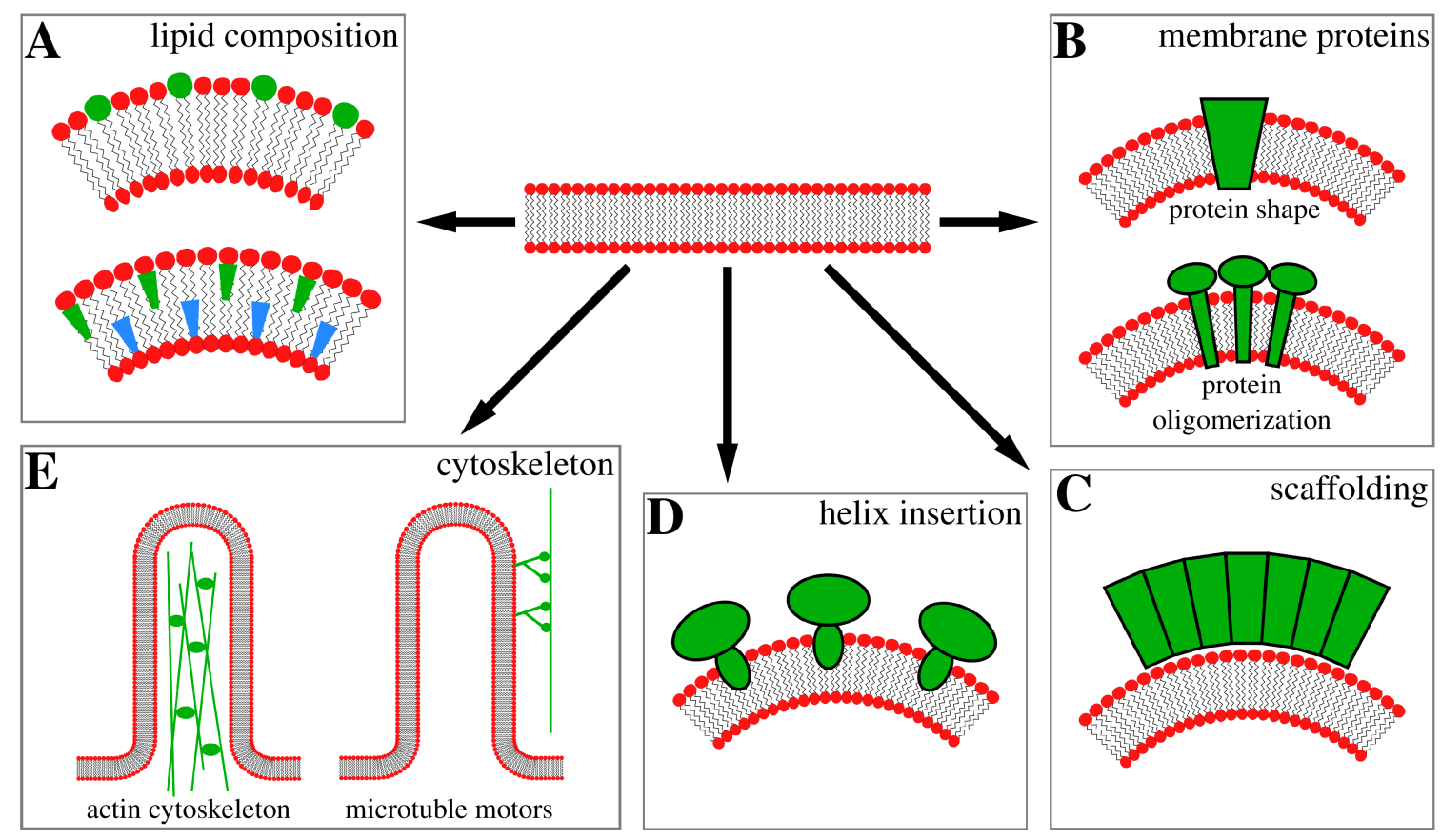

Figure 1.2: Schematic illustration of different mechanisms inducing membrane curvature. A: Spontaneous curvature of lipids. B: Insertion of proteins into the lipid bilayer. C: Proteins with scaffolding properties. D: Insertion of an amphipathic helix into the lipid monolayer. E: Interaction with the cytoskeleton. Derived from McMahon et al.3]

Proteins can bend lipid bilayers to their preferred curvature, when this is energetically more favorable then binding to a planar membrane (scaffolding, Figure 1.2 C). [10] Coating proteins, such as clathrin, COPI and COPII, polymerize and form a rigid cage shaping the membrane to a certain curvature which is 
then stabilized. [10, 11] Proteins of the dynamin family bind specifically to phosphoinititols, oligomerize and thereby mold membranes into highly curved tubular structures. By GTP dependent constriction of the oligomers, this leads to membrane scission. [3] BAR (Bin, Amphiphysin and Rvs) domains are banana-shaped proteins which dimerize and bind to the membranes through their concave shape. Hereby, the protein-membrane interaction is mainly mediated by electrostatics even though some BAR domains contain regions that bind to specific compartments. As the strength of electrostatic interactions increases with increasing contact area between membrane and protein, BAR domains maximize this contact area by sensing lipid bilayers matching their preferred curvature (curvature sensing). [3, 4] Some proteins belonging to the BAR superfamily, such as N-BAR, mold membranes not only by scaffolding but additionally bear one or more amphipathic helices. Proteins harboring amphipathic helices can induce large curvatures even though they do not have scaffolding properties. These helices can insert into a membrane monolayer inducing a local spontaneous curvature due to the generated asymmetry between the lipid monolayers. The curvature is then stabilized by the protein shape (Figure 1.2D). [12] Helices inserting into a membrane splay the lipid head groups, leading to a reorientation of the lipid tails. The protein epsin binds specifically to phosphatidylinositol-4,5-bisphosphate $\left(\mathrm{PIP}_{2}\right)$ being the first step in the formation of a clathrin coated pit. Due to the lack of a scaffold, the generated curvature must be stabilized differently. This can be achieved by proteins forming rigid cages such as clathrin. [13]

Despite the direct formation of membrane curvature by clustering of lipids and proteins, the actin-cytoskeleton shapes cell membranes during endocytosis, cell movement and division (Figure 1.2 E). ${ }^{3}$ It mediates the macroscopic curvature of the plasma membrane and maintains physical properties such as membrane tension. ${ }^{[14]}$ Membrane tension is directly related to the scaffolding properties of the actin-cytoskeleton and lowering the membrane tension has been shown to increase the endocytotic activity of cells. 15, 16,

A topic of current discussion is to what extent the described mechanisms contribute to the generation of membrane curvature. Especially, how protein crowding contributes to the generation of membrane curvature is highly debated. 


\subsection{Membrane remodeling in endocytosis}

During the formation of an endocytotic liposome, a planar membrane bends and forms a vesicle which detaches and transports an enclosed cargo. The proteins involved in the formation of the endocytotic vesicles depend on compartment where the liposomes are formed. Even though the different compartments and pathways fulfill diverse functions, the formation of endocytotic vesicles at the endoplasmatic reticulum (ER), the golgi network and the plasma membrane has been proposed by similar mechanisms presumably resulting from an evolutionary conserved mode of action. [10]

In the biosynthetic and recycling pathways between gogli and ER, vesicle formation is initiated by small G-proteins such as Sar1. Sar1 changes its conformation and inserts an $\alpha$-helix into the membrane which is believed being sufficient to induce membrane curvature. [11] Furthermore, Sar1 recruits the coating protein COPII which has been shown to induce membrane curvature and is expected to also stabilize the curved bilayers. In vivo, a rigid COPII cage is formed whereas the formation of tubular structures has been observed in vitro. ${ }^{[5]}$ Hereby, the tubulation of giant unilamellar vesicles (GUVs) was dependent on the lipid composition further amplifying the complex interplay of proteins and lipids in the generation of membrane curvature. Additionally, this suggests that the lipid composition is also involved in regulating the size and shape of the curved structures in vivo. Fission is then initiated by hydrolysis of GTP and blocked when GTP hydrolysis is inhibited. [17] Hereby, the exact mechanism and timing leading to bilayer separation remains a topic of current discussion. It was proposed that withdrawal of the amphipathic helix might initiate fission by forming membrane defects. [18] This pathway is supported by in vitro experiments with GUVs. COPII coated tubes found in these experiments were rigid and vesicle tubulation was dependent on the presence of the amphipathic helix of Sar1. Therefore, the COPII coat could provide the molecular driving force preventing the membrane to close the formed defects resulting from withdrawal of the Sar1 helix. [11]

At the plasma membrane, one of the best characterized endocytotic pathway is the clathrin mediated endocytosis which belongs to the class of micropinocytosis. It is responsible for the internalization of various receptors involved in cellular homeostasis, synaptic transmission and cell differentiation. If key proteins involved in clathrin mediated endocytosis malfunction this results in embryonic 
lethality. [19, 20] As a consequence, mutations of these proteins are not expected in human diseases. Nevertheless, many of the proteins were found being perturbed in cancer cells. 21] It is believed that clathrin dependent and clathrin independent endocytosis are equi-prevalent modes for internalization in fibroblastic cells. The number of different cargos internalized during clathrin mediated endocytosis is highly dependent on the cell type and ranges from only a few different compounds to more than twenty in synapses. [22] The cargo selection is achieved by the presence of different accessory cargo adaptor proteins ensuring the specific internalization of the receptors.

The first step in the formation of an endocytotic vesicle is the generation of a curved membrane bud from a planar membrane. Many proteins have been localized at regions with high curvature and even though lipid bilayers are often modeled as passive barriers, they also directly participate in regulating processes such as endocytosis. Different proteins such as epsin, the BAR protein superfamily and the ARF (ADP-ribosylation factor) family proteins are believed to induce membrane curvature. [22]

A

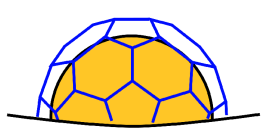

B

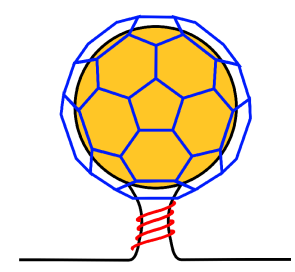

C

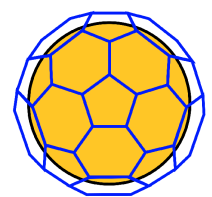

D

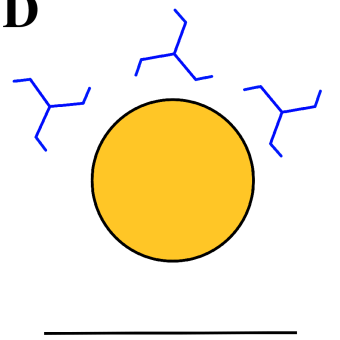

Figure 1.3: Schematic illustration of the formation and fission of an endocytotic vesicle. A: The planar membrane is curved and clathrin (blue) polymerizes around the membrane bud. B: The vesicle is fully coated with clathrin except for its neck where the scission protein dynamin (red) oligomerizes. C: The vesicle is separated from the plasma membrane. D: The clathrin coat depolymerizes. Derived from Doherty et al. 22.

After vesicle formation is initiated by binding of proteins such as epsin, the plasma membrane buds and clathrin triskelia polymerize into hexagons and pentagons coating the bud (Figure 1.3A). Subsequently, the clathrin coated pit evolves to a clathrin-coated vesicle with a wide range of curvatures. About 100 triskelia are required for the coating of an endocytotic vesicle. ${ }^{[21]}$ This process is regulated by a complex protein machinery. As clathrin does not bind directly to the membrane, adaptor proteins such as epsin and AP180 and accessory proteins such as AP2 
are required. These proteins are necessary for the formation of the clathrin lattice which then shapes and stabilizes the vesicle. After formation of the vesicle and the clathrin coat, the GTPase dynamin oligomerizes at the neck of the vesicle (Figure 1.3 B). Constriction of the vesicle neck leads to the separation of membrane and vesicle which is then released into the cell interior (Figure 1.3 C). ${ }^{[23]}$ Dynamin is recruited by proteins containing BAR domains, which preferentially bind to highly curved membrane regions such as the neck of the vesicle. [24] The clathrin coat is then depolymerized by the protein auxilin and the ATPase heat shock cognate 70 (HSC 70) and the vesicle undergoes further trafficking inside the cell before the cargo is delivered (Figure 1.3D). [22]

Even though the understanding of endocytosis has improved substantially during the last decades, specific questions remain unanswered. Until now it is unclear, how exactly the planar plasma membrane is curved which is the first step in the formation of an endocytotic coated vesicle. Additionally, the clathrin coat has been simulated to not provide enough energy to bend the membrane. ${ }^{[25]}$ Therefore, a topic of current discussion is whether proteins involved in endocytosis, such as epsin, actively generate membrane curvature and thereby facilitate the formation of a membrane bud. Also accessory proteins such as N-BAR are discussed being involved in the generation of membrane curvature. The time scale of the individual processes of endocytosis is also debated. The time span from vesicle formation until fission is regulated by membrane tension and area which can be controlled by interactions of the membrane with the actin-cytoskeleton. In vivo, membrane budding, vesicle formation and dynamin induced fission takes typically a few seconds up to minutes. [26] However, recently a new endocytotic pathway has been discovered being up to 200-fold faster than clathrin mediated endocytosis. 27] As it is unlikely that protein arrangement is fast enough to efficiently regulate this type of endocytosis, additional regulatory mechanisms are required. Membrane tension has been proposed as such a regulator. [28]

\subsection{Membrane tension as a regulator in endocytosis}

The shape of the plasma membrane of cells undergoes constant remodeling. Locally, regions of very high membrane curvature are generated either to initiate cellular processes or as a result of those processes. Hereby, the membrane does not act 
as a passive barrier but actively participates in these processes by adapting its composition, area and physical properties such as lateral tension.

Cells maintain a certain membrane area to regulate their volume and lateral tension. An increase in area does not necessarily changes the membrane tension as shown by fusion of giant vesicles with cells. Even though a large amount of lipid material was incorporated, only minor changes in membrane tension were detected. [29] Nevertheless, membrane area is often directly related to the lateral tension and both are actively regulated by the underlying cytoskeleton and the assembly and disassembly of caveolae (Figure 1.4. [30] It has been shown to regulate cell motility and vesicle trafficking. 29, 31, 32] Membrane tensions ranging from 0.003 to $0.45 \mathrm{mN} / \mathrm{m}$ were measured depending on the cell type and state. 29, 33, 35

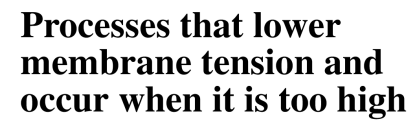

Exocytosis
Caveolae
disassembly

Stalling of the actin network
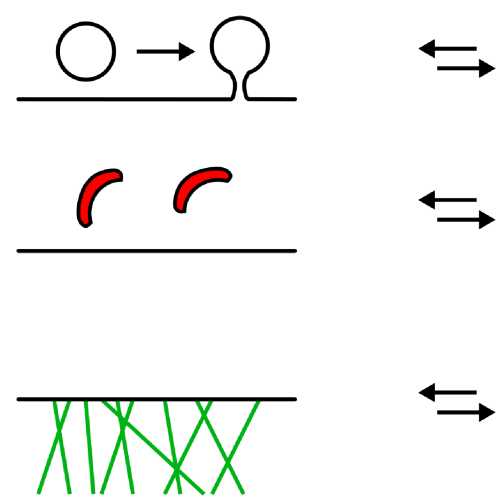

Processes that increase membrane tension and occur when it is too low

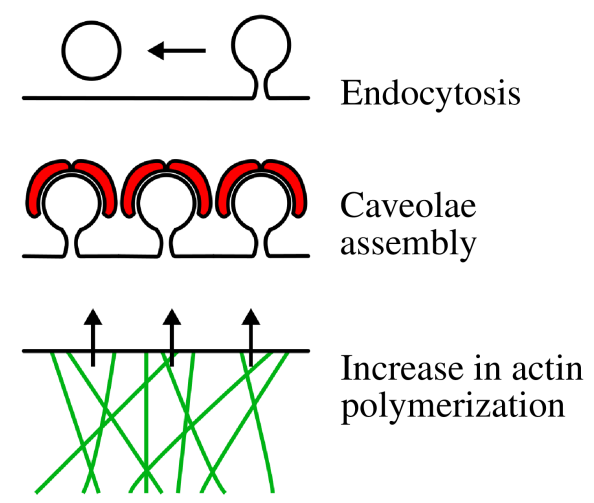

Figure 1.4: Schematic illustration of processes regulating membrane tension. Derived from Diz-Munoz et al. [30]

As a high membrane tension increases the elastic energy, it stimulates processes leading to a reduction of the membrane tension such as exocytosis. 36 Accordingly, processes such as endocytosis which remove lipid material thereby increasing the membrane tension, are facilitated by a low membrane tension. [16, 37] During the formation of an endocytotic vesicle, the plasma membrane is curved requiring to overcome a large energy barrier $\left(\approx 4 \cdot 10^{-19} \mathrm{~J}=10 k_{\mathrm{B}} T\right)$. ${ }^{38}$ The different proteins involved in clathrin mediated endocytosis generate the force necessary for membrane bending. Self-accelerating feedback mechanisms are believed to regulate the highly complex and energy dependent processes of clathrin mediated endocytosis. [30] The attachment of the actin cortex has been shown to regulate the plasma 
membrane tension. Actin polymerization generates a mechanical force of 1 to $2 \mathrm{pN}$ per filament via a Brownian ratchet mechanism. ${ }^{39}$ In yeast cells, actin polymerization is required for endocytosis and electron microscopy experiments revealed association of actin and clathrin coated structures. 40, 41] In contrast to yeast cells, actin polymerization during endocytosis is only required under stringent conditions in mammalian cells. Experiments with MDCK cells show that only at high membrane tension actin polymerization is triggered at the endocytotic sites to counteract the high membrane tension. Hereby, the authors suggested that actin decreases the energy necessary for the formation of a vesicle from the evaginated pit. The energy needed for the formation of a vesicle from an evaginated pit is significantly higher than the work required for the formation of an evaginated pit from a planar membrane. ${ }^{[42]}$ The identified interaction of actin and epsin provided the molecular basis for the relation of membrane tension and endocytosis in yeast. If the force required for the formation of a vesicle is too high, epsin binds to actin through a binding site at the C-terminus of epsin. [4] This clearly shows how protein-protein interactions, which are related to mechanical properties such as membrane tension, regulate endocytosis. However, the triggers and spatio-temporal sequence of these processes still need to be elucidated.

\subsection{Epsin N-terminal homology domain}

The protein epsin is one of the best characterized proteins in clathrin mediated endocytosis. Discovered in 1998, it was shown to be expressed in all vertebrates and many cells. [4] Epsin interacts with various binding partners and its C-terminus is involved in binding of the proteins actin, clathrin and others. Polymerization of clathrin leads to the formation of the clathrin coat of the endocytotic vesicles determining their shape and size.

The approximately 150 amino acids long domain at the N-terminus of epsin (epsin $\mathrm{N}$-terminal homology domain, ENTH) is evolutionary well preserved. Structurally related proteins were identified by sequence alignment in different organisms such as frogs, plants, yeast and humans. ${ }^{[45]}$ ENTH consists of seven $\alpha$-helices forming a super helix (Figure 1.5 A) and binds specifically to phosphatidylinositols with the greatest affinity to PIP 2 . The crystal structure of ENTH bound to inositol-1,4,5trisphosphate $\left(\mathrm{I}(1,4,5) \mathrm{P}_{3}\right)$ revealed a coordination of all phosphates by different 
residues from the helices zero, one, three and four and from the loop between helix one and two. [13, 46] Upon binding to its receptor lipid, a previously unstructured part of ENTH forms an $\alpha$-helix (helix zero) which inserts into the membrane (Figure 1.5 B). [13, 47]

A

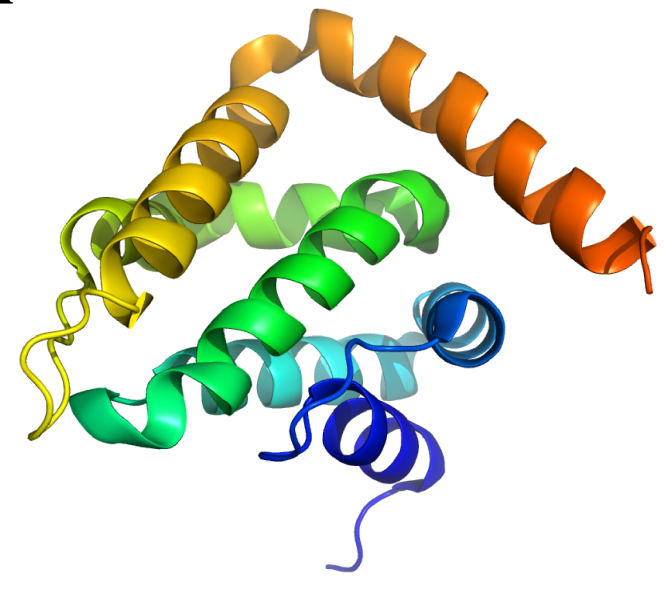

B

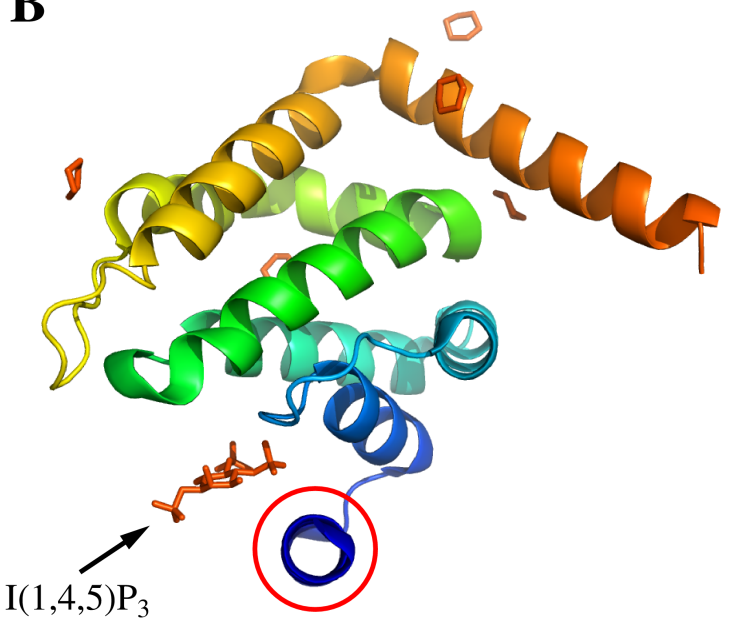

Figure 1.5: A: Crystal structure of the epsin N-terminal homology domain (ENTH). 48] B: Crystal structure of the ENTH-I $(1,4,5) \mathrm{P}_{3}$ complex. ${ }^{[49]}$ Compared to unbound ENTH, a new helix has forms upon binding (red circle).

The formed helix is amphipathic having hydrophobic residues on its membranefacing surface and coordinating the lipid head groups with its cytosol-facing surface. The affinity of ENTH to PIP $_{2}$ ranges from $2.6 \mathrm{~nm}$ to $0.85 \mu \mathrm{M}$ depending on the experimental conditions such as lipid and buffer composition, $\mathrm{pH}$ and receptor lipid concentration. [13, 50, 51] The insertion of the amphipathic helix into the membrane generating an asymmetry between the monolayers is believed to be responsible for the generation of membrane curvature and budding of the plasma membrane. ENTH has been shown to cause tubulation and vesiculation of giant unilamellar vesicles. ${ }^{8}$, 47, 52] The membrane remodeling ability of ENTH is dependent on the hydrophobic character of the outer part of the helix. When leucin at position 6 (L6) was replaced with a more polar glutamine (L6Q) or glutamic acid (L6E) residue, the ability to tubulate GUVs was reduced. Mutation of this position to a more hydrophobic tryptophan residue (L6W) resulted in increased tubulation and vesiculation activity. [13, 52]

Additionally to the epsin N-terminal homology domain, similar N-terminal homology domains are found in several other proteins. The proteins of the AP180/CALM 
family have a structurally similar domain at their N-terminus (ANTH). Adaptor proteins (AP) are a major component of neuronal endocytotic clathrin coats and CALM is the non-neuronal homolog. [4]] In contrast to ENTH, ANTH does not form a new helix upon binding to PIP $_{2}$. Instead, binding is mediated by lysine residues in $\alpha$-helix one and $\alpha$-helix two. An important difference between ANTH and ENTH is the missing ability of ANTH to generate membrane curvature when binding to $\mathrm{PIP}_{2} \cdot{ }^{[4]}$ As epsin and the protein AP2 have been identified both binding to PIP 2 and to each other, a cooperative mechanism has been proposed. This indicates that multiple interactions mediate and regulate ENTH and ANTH binding to the membrane and that the formation of protein-protein networks lead to the recruitment of a stable clathrin coat. ${ }^{[53]}$ However, the precise spatial and temporal regulation of this process is still unclear and needs to be investigated. 



\section{Scope of thesis}

During endocytosis, a flat membrane is deformed and a highly curved vesicle is formed. This complex process is precisely regulated by a complex protein machinery and physical properties such as membrane tension. The protein epsin is one of the essential proteins involved in clathrin mediated endocytosis. Upon binding to its receptor lipid phosphatidylinositol-4,5-bisphosphate ( $\left.\mathrm{PIP}_{2}\right)$, its $\mathrm{N}$-terminal region restructures and forms an $\alpha$-helix, which inserts into the membrane. Up to now, little is known about the mechanistic origin of the curvature generation and initiation of the formation of an endocytotic vesicle.

For this reason, artificial model membranes are used to study binding and activity of the epsin N-terminal homology domain (ENTH) in a controlled environment. The membrane remodeling ability of ENTH will be analyzed to investigate how it changes as a function of lateral tension and lipid composition. Additionally, it will be studied if the mechanical properties of the membrane are altered by binding of ENTH. As a first step, the dissociation constant of ENTH binding to $\mathrm{PIP}_{2}$ will be determined for different lipid compositions and membrane topologies to investigate whether the binding affinity is changed by membrane curvature or lateral tension.

Protruded pore-spanning membranes will be established to study the remodeling ability of ENTH as a function of lipid composition. Lipid bilayers with different area compressibility moduli and lysis tensions will be analyzed to answer how these membrane properties alter the activity of ENTH.

Controlling the adhesion strength of the giant unilamellar vesicles (GUVs) to a solid surface will allow tuning their lateral tension. This enables to study the membrane remodeling ability of ENTH as a function of lateral tension and to contribute to the debate whether cells use membrane tension as a regulator to suppress or promote the formation of highly curved structures. 



\section{Materials and methods}

\subsection{Osmotic pressure}

Cellular membranes are almost impermeable barriers for ions and polar solutes, but more permeable to water. Different concentrations of solutes between cell interior and exterior lead to exchange of water through the cell membrane. The exchange of water through such a semi-permeable membrane gives rise to an osmotic pressure $\Pi$. The osmotic pressure is defined as the pressure required to maintain the concentration gradient and to avoid movement of water across the membrane. It belongs to the class of colligative properties and is dependent on the concentration difference between both sides of the membrane but not on the type of solutes.

The osmotic pressure at equilibrium is given by the equilibrium of the chemical potentials $\mu$. Compared to the chemical potential of a pure phase, the chemical potential of an ideal binary mixture of compounds $a$ and $b$ is reduced by the fraction $x_{a}$ of compound $a$ and increased by pressure $\Pi$. At equilibrium pressure and constant temperature the equilibrium is given by:

$$
-R T \ln x_{a}=\int_{p}^{p+\Pi} V_{m} \mathrm{~d} p
$$

with $V_{m}$ being the molar volume, $T$ the absolute temperature and $R$ the universal gas constant. 
Substitution of $x_{a}=1-x_{b}$ and approximating $\ln \left(1-x_{b}\right) \approx-x_{b}$ for small $x_{b}$ yields:

$$
R T x_{b}=\int_{p}^{p+\Pi} V_{m} \mathrm{~d} p .
$$

As the pressure range for integration is small the molar volume is assumed as constant and integration gives:

$$
R T x_{b}=V_{m} \Pi
$$

For dilute mixtures $\left(x_{b} \ll x_{a}\right)$ one finds $x_{b} \approx n_{b} / n_{a}$. Introducing the Van't Hoff factor $i$, representing the number of molecules after dissociation, leads to the Van't Hoff equation which describes the osmotic pressure of an ideal mixture $\Pi_{\text {id }}$ (equation 3.4) as a function of temperature and molar concentration $c$.

$$
\Pi_{\mathrm{id}}=i c R T
$$

At high concentrations of solutes the real osmotic pressure $\Pi_{\text {real }}$ differs from the expected ideal behavior. The osmotic coefficient $\phi$ is then used as a correction factor to account for the differences between real and ideal osmotic pressure $\Pi_{\text {id }}$ (equation 3.5).

$$
\phi=\frac{\Pi_{\text {real }}}{\Pi_{\text {id }}}
$$

The osmolarity $O$ is then calculated by:

$$
O=\frac{\Pi_{\text {real }}}{R T}=i c \phi
$$

As the osmotic coefficient is also a function of the concentration, a virial expansion can be used to correct the osmotic pressure as a function of the molar concentration (equation 3.7).

$$
\Pi_{\text {real }}=i c R T\left(1+B c+C c^{2}+\ldots\right)
$$

The coefficients $B$ and $C$ are compound specific virial coefficients which are used to correct for the non-ideal behavior. 


\subsubsection{Determination of the osmolality by freezing point depression}

The phase transition of an ideal binary mixture is given by the equilibrium of the chemical potentials of a pure solid phase $\alpha$ and a mixed liquid phase $\beta$. The presence of an additional compound in the liquid phase decreases the chemical potential $\mu$ which is given by equation 3.8 .

$$
\mu_{a}(T, p)=\mu_{a}^{*}(T, p)+R T \ln x_{a} .
$$

The phase transition can be assumed to be pressure independent. As it only occurs for the solvent (component $a$ ) and as the solid phase of the solvent is a pure phase the equilibrium condition is given by:

$$
\mu_{a}^{* \alpha}=\mu_{a}^{\beta} .
$$

Combination of equation 3.8 and 3.9 yields:

$$
\mu_{a}^{* \alpha}=\mu_{a}^{* \beta}+R T \ln x_{a},
$$

which can be rearranged to:

$$
\ln x_{a}=\frac{\mu_{a}^{* \alpha}-\mu_{a}^{* \beta}}{R T}=-\frac{\Delta_{\mathrm{m}} G}{R T},
$$

with $\Delta_{\mathrm{m}} G$ being the Gibbs free enthalpy. The osmolality of a solution can be measured by freezing point depression. The isobaric change of the chemical potential with temperature is given by equation 3.12 .

$$
\frac{\mathrm{d} \ln x_{a}}{\mathrm{~d} T}=\frac{\Delta_{\mathrm{m}} H}{R T^{2}},
$$

where $\Delta_{\mathrm{m}} H$ is the melting enthalpy of the solvent. Integration of equation 3.12 from the melting temperature of the mixture $T_{\mathrm{m}}$ to the melting temperature of the pure solvent $T_{\mathrm{m}}^{*}$ and from 0 to $\ln x_{a}$ yields:

$$
\int_{T_{\mathrm{m}}}^{T_{\mathrm{m}}^{*}} \frac{\Delta_{\mathrm{m}} H}{R T^{2}} \mathrm{~d} T=\int_{0}^{\ln x_{a}} \mathrm{~d} \ln x_{a} .
$$


Assuming the melting enthalpy being temperature independent, the integral in equation 3.13 is solved to:

$$
\frac{\Delta_{\mathrm{m}} H}{R}\left(\frac{1}{T_{\mathrm{m}}^{*}}-\frac{1}{T_{\mathrm{m}}}\right)=\ln x_{a} .
$$

For an ideal binary mixture the fraction $x_{a}$ is given by $x_{a}=1-x_{b}$. For dilute solutions the approximation $\ln \left(1-x_{b}\right) \approx-x_{b}$ can be made and rearrangement of equation 3.14 leads to:

$$
\frac{\Delta_{\mathrm{m}} H}{R}\left(\frac{1}{T_{\mathrm{m}}^{*}}-\frac{1}{T_{\mathrm{m}}}\right)=\frac{\Delta_{\mathrm{m}} H}{R}\left(\frac{T_{\mathrm{m}}-T_{\mathrm{m}}^{*}}{T_{\mathrm{m}} T_{\mathrm{m}}^{*}}\right) \approx \frac{\Delta_{\mathrm{m}} H}{R}\left(\frac{T_{\mathrm{m}}-T_{\mathrm{m}}^{*}}{T_{\mathrm{m}}^{* 2}}\right)=-x_{b} .
$$

Equation 3.15 can be rearranged to:

$$
T_{\mathrm{m}}-T_{\mathrm{m}}^{*}=-\frac{R T_{\mathrm{m}}^{* 2}}{\Delta_{\mathrm{m}} H} x_{b}
$$

The molar fraction of component $b$ of an ideal binary mixture of $a$ and $b$ and its molality $c_{b}^{*}$ are related by:

$$
x_{b}=\frac{M_{a} c_{b}^{*}}{1+M_{a} c_{b}^{*}},
$$

with $M_{a}$ being the molar mass of the solvent. For large dilutions equation 3.17 simplifies to:

$$
x_{b}=M_{a} c_{b}^{*}
$$

Combination of equation 3.16 and 3.18 leads to the freezing point depression given by:

$$
T_{\mathrm{m}}-T_{\mathrm{m}}^{*}=-\frac{R T_{\mathrm{m}}^{* 2} M_{a}}{\Delta_{\mathrm{m}} H} c_{b}^{*}
$$

with

$$
K_{a}=\frac{R T_{\mathrm{m}}^{* 2} M_{a}}{\Delta_{\mathrm{m}} H}
$$

being the cryoscopic constant $K_{a}$. The molality $c^{*}$ and osmolality $O^{*}$ are related by the Van't Hoff factor $i\left(O^{*}=c^{*} \cdot i\right)$ and the osmolality of an ideal mixture measured by freezing point depression is then given by:

$$
O^{*}=\frac{\left(T_{\mathrm{m}}^{*}-T_{\mathrm{m}}\right)}{K_{a}} .
$$


The conversion of molal and molar concentration is given by equation 3.22

$$
c=\frac{c^{*} \rho_{\text {solution }}}{1+c^{*} M \cdot 10^{-3}},
$$

with $\rho_{\text {solution }}$ being the solution density, $M$ the molar mass and the factor of $10^{-3}$ accounting for unit conversion from volume to mass. ${ }^{[54]}$ As it is complicated to measure the density of the solution with high precision and as the partial volume of the osmolyte depends only little on the osmolyte concentration the molarity can also be calculated using equation 3.23 .

$$
c \approx \frac{c^{*} \rho_{\text {solvent }}}{1+c^{*} \frac{\rho_{\text {solvent }}}{c_{\max }}},
$$

with $\rho_{\text {solvent }}$ being the density of the solvent and $c_{\max }$ the molarity of the pure osmolyte. For $c^{*} \ll c_{\max }$ and water as solvent $(\rho \approx 1 \mathrm{~kg} / \mathrm{L})$ equation 3.23 simplifies to:

$$
c \approx c^{*}
$$

\section{Calibration of the osmometer}

Different masses of osmolytes were added to known masses of water $(\approx 50 \mathrm{~g})$ to generate solutions with osmolalities ranging from 0 to $400 \mathrm{mOsmol} / \mathrm{kg}$. The theoretical osmolality $O_{\text {theo }}^{*}$ was calculated based on the determined masses of water $m_{\text {solvent }}$ and osmolyte $m_{\mathrm{o}}$ using literature values for the osmotic coefficient $\phi$ (equation 3.25, [55, 56]

$$
O_{\text {theo }}^{*}=\frac{m_{\mathrm{o}} M_{\mathrm{o}} i \phi}{m_{\text {solvent }}}
$$

A volume of $65 \mu \mathrm{L}$ of the solution of interest was measured by freezing point depression using an Osmomat 030 (Gonotec, Berlin, Germany). Five measurements were averaged for each solution and the measured osmolality was then plotted vs. the calculated theoretical osmolality. Linear fitting of the data obtained for $\mathrm{NaCl}$ with $O^{*}=b \cdot O_{\text {theo }}^{*}$ yielded a high correlation coeffiecient of $R^{2}=0.9998$ and a slope of $b=1.01$ showing the consistency of theoretical and experimentally measured osmolality (Figure 3.1A). For sucrose solutions increasing deviations from the theoretical osmolality were measured with increasing sucrose concentration. Sweeney et al. determined deviations between measured and calculated 
osmolality increasing from $5 \%$ for a 0.2 Osmol $/ \mathrm{kg}$ sucrose solution to $9.4 \%$ for a sucrose solution with $2 \mathrm{Osmol} / \mathrm{kg}$. [57 Violations of the thermodynamic assumptions, such as the cryoscopic constant being independent of temperature and osmolyte concentration, are possible explanations for the observed deviations. As the cryoscopic constant increases from $1.86 \mathrm{~K} \mathrm{~kg} \mathrm{~mol}^{-1}$ to $\approx 2.25 \mathrm{~K} \mathrm{~kg} \mathrm{~mol}^{-1}$ for a $2 \mathrm{Osmol} / \mathrm{kg}$ sucrose solution, the measured osmolality is overestimated by the osmometer device which uses a constant cryoscopic constant of $1.86 \mathrm{~K} \mathrm{~kg} \mathrm{~mol}^{-1}$. Since it was possible to accurately determine the theoretical osmolarity, as shown for $\mathrm{NaCl}$, the data measured for sucrose were fitted with $\mathrm{O}^{*}=B^{2} \cdot \mathrm{O}_{\text {theo }}^{*}+C \cdot O_{\text {theo }}^{*}$ and used as a calibration of the osmometer device. Fitting yielded $B=0.5885$ and $C=0.97515$ with $R^{2}=0.9999$.
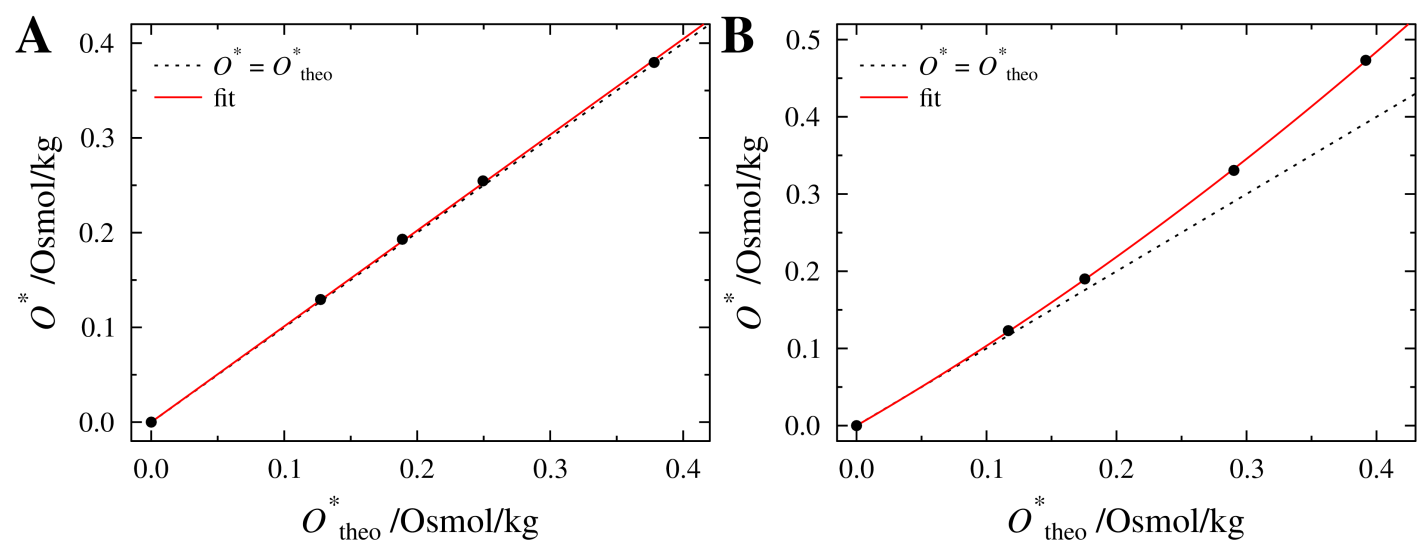

Figure 3.1: Plot of measured osmolality vs. theoretical osmolality for A: $\mathrm{NaCl}$ and $\mathbf{B}$ : sucrose.

\subsection{Lipids}

The plasma membrane of eukaryotic cells is a highly complex barrier mainly composed of lipids, proteins and cholesterol. It is attached to an actin-cytoskeleton and separates the cell interior from the external environment. Artificial membranes were used to mimic the plasma membrane reducing the level of complexity. Different lipid compositions were used as the mechanical properties of lipid bilayers, which are used to regulate biological processes, are influenced by the chemical structure of the lipids. 


\section{Matrix lipids}

The matrix lipids used all belong to the class of phospholipids, the major component of the plasma membrane. ${ }^{[58]}$ They have a glycerol backbone bearing the hydrophilic lipid head group and two fatty acids, representing the hydrophobic part of the lipid. 1-palmitoyl-2-oleoyl-sn-glycero-3-phosphocholine (POPC, Scheme 3.1. $\mathrm{C}_{42} \mathrm{H}_{82} \mathrm{NO}_{8} \mathrm{P}, M=760.08 \mathrm{~g} / \mathrm{mol}$ ) is highly accumulated in the plasma membrane. It has a zwitterionic choline head group and the glycerol backbone is esterified with palmitic acid and oleic acid. Oleic acid has a cis-double bond at position $\Delta^{9}$ which causes a kink in the membrane and disturbs the packing of lipids. POPC has a low spontaneous monolayer curvature of $J_{0}^{\mathrm{m}}=-0.02 \mathrm{~nm}^{-1}$ and forms preferentially planar membranes. [59]

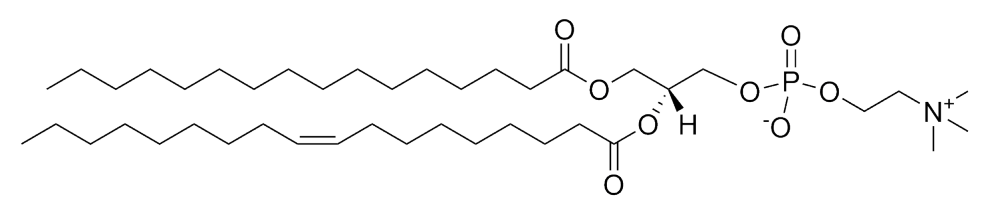

Scheme 3.1: Structure of POPC.

Another major component of the plasma membrane is 1-palmitoyl-2oleoyl-sn-glycero-3-phosphoethanolamine (POPE, Scheme 3.2, $\mathrm{C}_{39} \mathrm{H}_{76} \mathrm{NO}_{8} \mathrm{P}$, $M=717.00 \mathrm{~g} / \mathrm{mol}$ ) which is structurally related to POPC. Instead of a tertiary methyl functionalized amin the head group bears a primary amine. Thus, the head group is smaller compared to the choline head group of POPC and thereby the spontaneous monolayer curvature is more negative $\left(J_{0}^{\mathrm{m}}=-0.32 \mathrm{~nm}^{-1}\right)$ caused by the smaller head group size. [59]

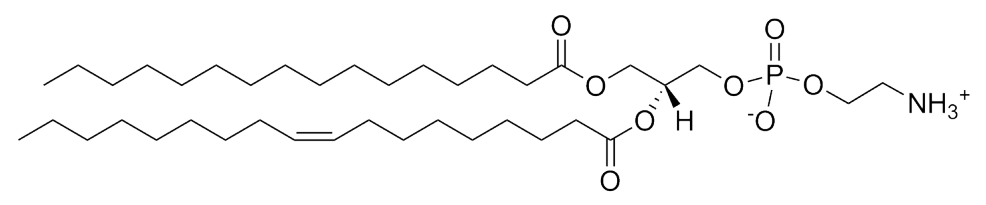

Scheme 3.2: Structure of POPE.

The synthetic phospohlipid 1,2-dioleoyl-sn-glycero-3-phosphocholine (DOPC, Scheme 3.3. $\mathrm{C}_{44} \mathrm{H}_{84} \mathrm{NO}_{8} \mathrm{P}, M=786.15 \mathrm{~g} / \mathrm{mol}$ ) with a glycerol backbone carries two 
oleic acids and a zwitterionic choline head group. Both fatty acids have a cis-double bond at $\Delta^{9}$ position. As both fatty acids are unsaturated with a cis double bond the spontaneous monolayer curvature of $J_{0}^{\mathrm{m}}=-0.09 \mathrm{~nm}^{-1}$ is slightly larger than for POPC. [59]

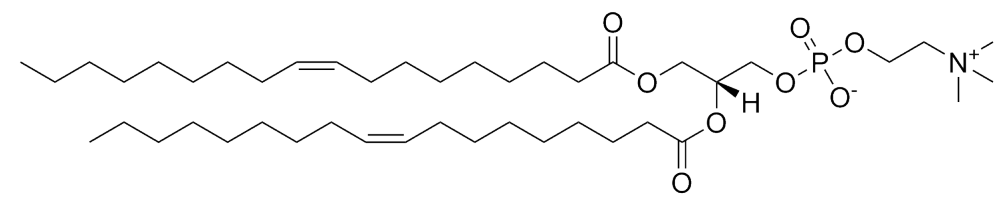

Scheme 3.3: Structure of DOPC.

1,2-dioleoyl-sn-glycero-3-phosphoethanolamine (DOPE, Scheme 3.4, $\mathrm{C}_{41} \mathrm{H}_{78} \mathrm{NO}_{8} \mathrm{P}$, $M=744.03 \mathrm{~g} / \mathrm{mol}$ ) is structurally related to DOPC as POPE to POPC. DOPE has a zwitterionic ethanol amine head group which is smaller than a choline head group. The combination of the small head group and two unsatturated fatty acids which enlarge the area of the hydrophobic part of the lipid results in the highest spontaneous monolayer curvature $\left(J_{0}^{\mathrm{m}}=-0.40 \mathrm{~nm}^{-1}\right)$ of POPC, POPE, DOPC and DOPE.

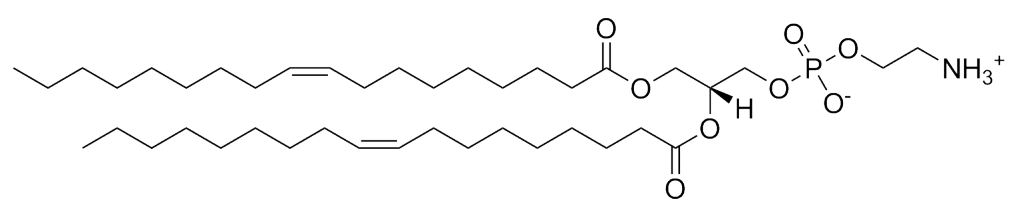

Scheme 3.4: Structure of DOPE.

The zwitterionic lipid 1,2-diphytanoyl-sn-glycero-3-phosphocholine (DPhPC, Scheme 3.5, $\mathrm{C}_{48} \mathrm{H}_{96} \mathrm{NO}_{8} \mathrm{P}, M=846.25 \mathrm{~g} / \mathrm{mol}$ ) also belongs to the group of phospholipids. It has a choline head group and glycerol backbone. DPhPC is found in membranes of archaea, which must withstand extreme conditions such as low $\mathrm{pH}$, high salt concentrations and high temperature. It has no phase transition temperature and its fatty acids are branched by methyl groups. [60] Membranes composed of DPhPC are mechanically stable as the branched lipid tails build intertwined networks. 61, 62, 


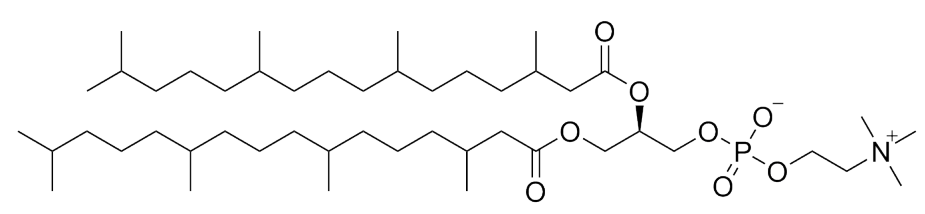

Scheme 3.5: Structure of DPhPC.

1,2-dipalmitoyl-sn-glycero-3-phosphoethanolamine-N-(cap biotinyl) is an analog to DOPE with a biotin functionalized headgroup (cap-biotin-DOPE, Scheme 3.6, $\mathrm{C}_{53} \mathrm{H}_{98} \mathrm{~N}_{4} \mathrm{O}_{11}$ PNaS, $M=1053.39 \mathrm{~g} / \mathrm{mol}$ ). It was used for specific binding of membrane containing this lipid to the protein avidin. The absence of a spacer between lipid and biotin leads to a close contact of membranes with this biotinylated lipid and the bound avidin molecules.

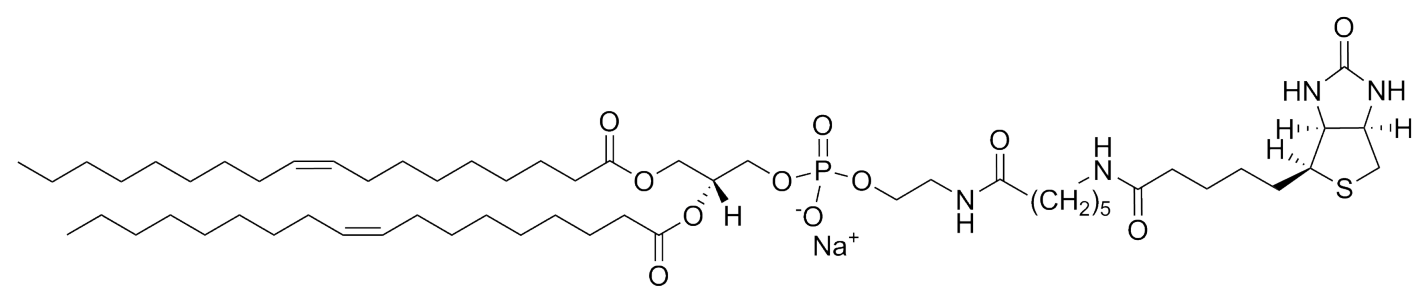

Scheme 3.6: Structure of cap-biotin-DOPE.

\section{Cholesterol}

Cell membranes contain up to $50 \%$ Cholesterol (Chol, Scheme 3.7, $\mathrm{C}_{27} \mathrm{H}_{46} \mathrm{O}$, $M=386.65 \mathrm{~g} / \mathrm{mol}$ ) which regulates the membrane fluidity. ${ }^{[58,63}$ Cholesterol has been shown to increase the mechanical stability of membranes as it increase the area compressibility modulus and the membrane's lysis tension. 64.65] Additionally, it has been shown to accumulate in regions with high membrane curvature. 66

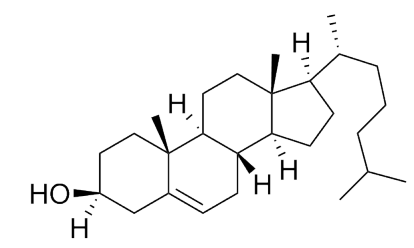

Scheme 3.7: Structure of cholesterol. 
L- $\alpha$-phosphatidylinositol-4,5-bisphosphate (ammonium salt)

The lipid L- $\alpha$-phosphatidylinositol-4,5-bisphosphate ( $\mathrm{PIP}_{2}$, Scheme 3.8, $\mathrm{C}_{47} \mathrm{H}_{94} \mathrm{~N}_{3} \mathrm{O}_{19} \mathrm{P}_{3}, \bar{M}=1096.36 \mathrm{~g} / \mathrm{mol}$ ) functions as a receptor for various proteins and is involved in membrane movement, actin-cytoskeletal assembly and membrane trafficking. ${ }^{67}$ Even though phosphatidylinositols are non-uniformly distributed in the plasma membrane and on trafficking vesicles, their concentration in mammalian cells is mostly below $10-15 \%$ of the total lipid content. Many proteins metabolizing $\mathrm{PIP}_{2}$ have been linked to human diseases. 68]

For the experiments conducted in this thesis, brain extracted $\mathrm{PIP}_{2}$ was used. The major fatty acids found in this extract are stearoylic and arachidonoylic acid. $\mathrm{PIP}_{2}$ has a negative charge of -4 at $\mathrm{pH} 7$ and is known to form clusters in the presence of divalent cations such as $\mathrm{Mg}^{2+}$ and $\mathrm{Ca}^{2+} .69$

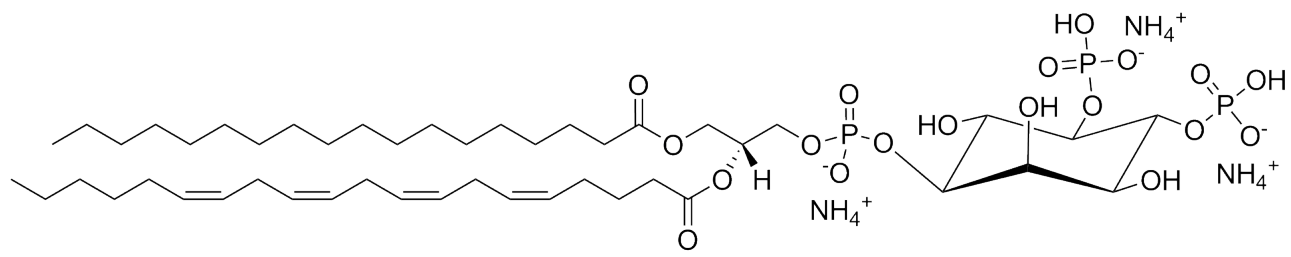

Scheme 3.8: Structure of $\mathrm{PIP}_{2}$.

\section{Fluorophores}

For imaging with fluorescence microscopy, the membranes were doped with lipids bearing a fluorophore. The properties of the fluorophores used for labeling are shown in Table 3.1. Except for PIP 2 , the fluorescent part of the lipid was located at the head group in order not to alter the hydrophobic interactions between the lipids. The fluorescent analog $\mathrm{PIP}_{2}$ is labeled with Bodipy-TMR at the $s n-1$ position. Head group-labeled PIP 2 was not used as interactions of $\mathrm{PIP}_{2}$ and proteins often involve the coordination of parts of the inositol group. To observe binding of ENTH to the membranes, primary amine labeling using a tetrafluorophenyl (TFP) ester of Alexa Fluor 488 carboxylic acid or cysteine labeling using Atto594-maleimide was conducted. 
Table 3.1: Properties of compounds used as markers for fluorescence microscopy.

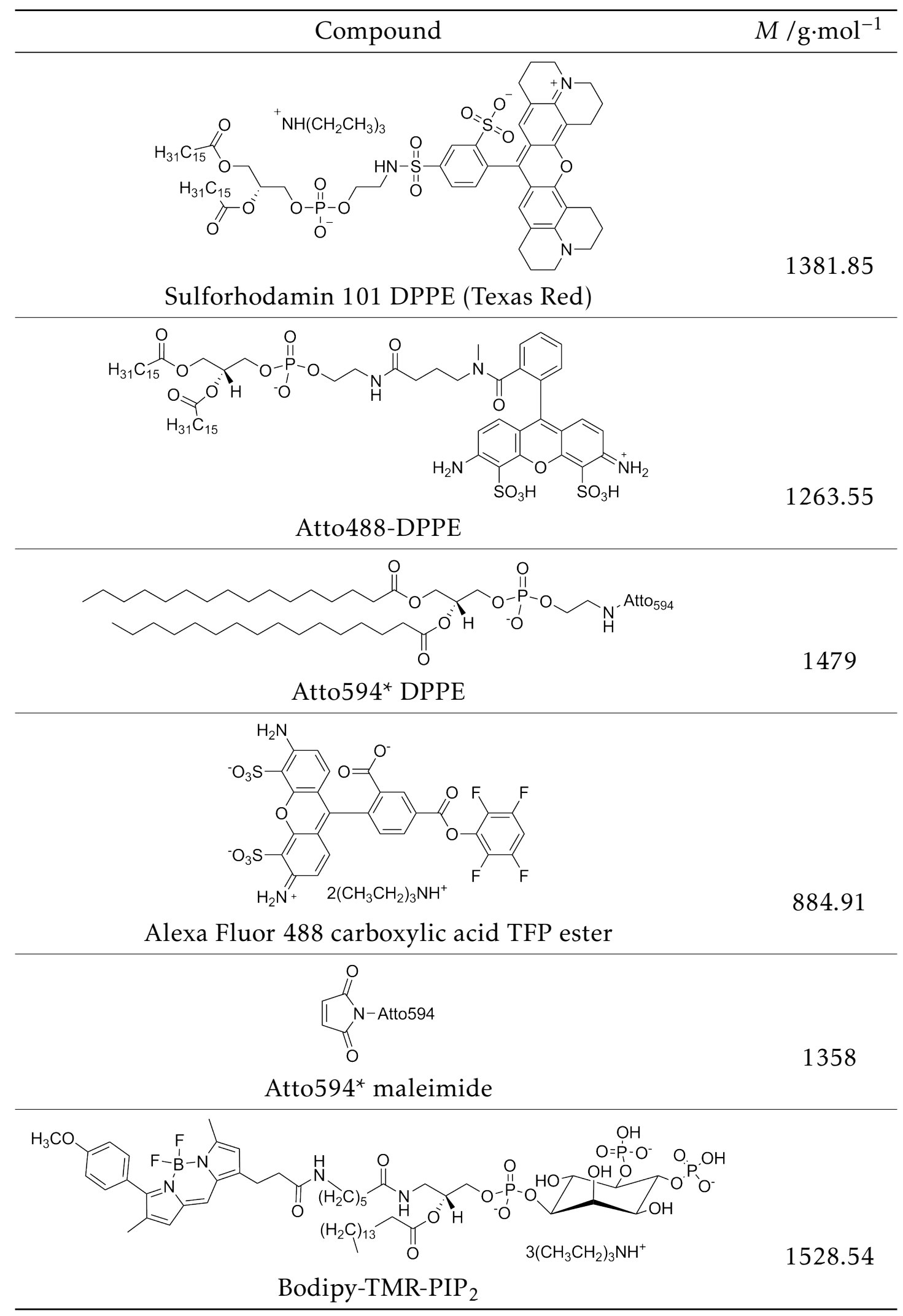

${ }^{*}$ structure of Atto594 is not published. 


\subsection{Proteins}

\subsubsection{Epsin $\mathrm{N}$-terminal homology domain}

The epsin N-terminal homology domain (ENTH) is an evolutionary highly conserved domain present in many endocytotic proteins. The ENTH domain of epsin, which is involved in clathrin mediated endocytosis, is composed of approximately 150 amino acids forming seven $\alpha$-helices. ${ }^{[44]}$ Upon binding to its receptor lipid PIP ${ }_{2}$, a new $\alpha$-helix assembles and inserts into the lipid bilayer generating a asymmetry between the monolayer inducing membrane curvature. 13.

\section{Experimental procedure}

ENTH used in this thesis was provided by Benjamin Kroppen from the group of Prof. Dr. Michael Meinecke (European Neuroscience Institute, Göttingen, Germany) and stored at $-80^{\circ} \mathrm{C}$. Directly before usage, the osmolarity of the protein buffer (150 mm NaCl, $50 \mathrm{~mm}$ HEPES, pH 7.4) was adjusted to the osmolarity of the buffer used for the experiment by dilution with water. For the experiments with giant unilamellar vesicles the protein was stored in sucrose buffer $(259 \mathrm{~mm}$ sucrose, $2 \mathrm{~mm} \mathrm{MgCl}_{2}, 2 \mathrm{~mm}$ TRIS, pH 7.4, $264 \mathrm{mOsmol} / \mathrm{L}$ ) at $-80^{\circ} \mathrm{C}$. Directly before usage, the $\mathrm{MgCl}_{2}$ concentration of the buffer was adjusted to the desired $\mathrm{Mg}^{2+}$ concentration by diluting with $\mathrm{Mg}^{2+}$-free sucrose buffer ( $262 \mathrm{~mm}$ sucrose, $2 \mathrm{~mm}$ TRIS, $\mathrm{pH} 7.4,264 \mathrm{mOsmol} / \mathrm{L})$. Properties of ENTH were calculated based on its sequence (Appendix, Section A.5, 48, 70]

\section{Labeling of ENTH}

To correlate changes in membrane topology to ENTH binding, the protein was labeled with a fluorescent probe. Two dyes with different spectral properties were used for labeling. For labeling of ENTH with Alexa Fluor 488, $50 \mu \mathrm{L} \mathrm{NaHCO}_{3}$ $(1 \mathrm{M})$ were added to $400 \mu \mathrm{L}$ of ENTH $(c=101 \mu \mathrm{M})$. An $1.3 \times$ excess of Alexa Fluor 488 carboxylic acid TFP ester $\left(\lambda_{\mathrm{ex}}=494 \mathrm{~nm}, \lambda_{\mathrm{em}}=519 \mathrm{~nm}, C F=0.11\right.$, $\epsilon=7.2 \cdot 10^{4} \mathrm{M}^{-1} \mathrm{~cm}^{-1}$, Invitrogen Ltd, Paisley, UK) was then added and the mixture was stirred for $1 \mathrm{~h}$ at room temperature. ${ }^{71}$ Size exclusion utilizing a G-25 DNA 
Grade column (GE Healthcare UK Limited, Little Chalfont, UK) was conducted for purification and removal of unbound dye.

For labeling of ENTH with Atto594, an 1 to $10 \times$ excess of Atto594-maleimide $\left(\lambda_{\mathrm{ex}}=603 \mathrm{~nm}, \lambda_{\mathrm{em}}=622 \mathrm{~nm}, C F=0.50, \epsilon=1.2 \cdot 10^{5} \mathrm{M}^{-1} \mathrm{~cm}^{-1}\right.$, ATTO-TEC GmbH, Siegen, Germany) was added to 100 to $395 \mu \mathrm{L}$ of ENTH (315 to $523 \mu \mathrm{M})$ and the mixture was stirred overnight at $4{ }^{\circ} \mathrm{C}$. ${ }^{72]}$ For removal of unbound dye, a G-25 DNA Grade column (GE Healthcare UK Limited, Little Chalfont, UK) was used. The concentration of the labeled protein was calculated using equation 3.26 with $A_{280}$ being the absorption of the protein at $280 \mathrm{~nm}, A_{\max }$ the absorption of the dye at its maximum emission wavelength, $C F$ being the correction factor for the dye absorption at $280 \mathrm{~nm}, M_{\mathrm{ENTH}}$ the molar mass of ENTH and $\epsilon_{\mathrm{ENTH}}$ and $\epsilon_{\text {dye }}$ being the molar extinction coefficient of the protein and the dye, respectively.

$$
c=\frac{A_{280}-A_{\max } \cdot C F}{\epsilon} M_{\mathrm{ENTH}}
$$

The absorption spectra were measured by UV/VIS spectroscopy (NanoDrop 2000c, Thermo Scientific, Wilmington, USA) and the degree of labeling (DOL) was calculated using equation 3.27 .

$$
D O L=\frac{A_{\max } \cdot \epsilon_{\mathrm{ENTH}}}{\left(A_{280}-A_{\max } \cdot C F\right) \epsilon_{\mathrm{dye}}}
$$

\subsubsection{Avidin}

The tetrameric glycoprotein avidin $(M=66 \mathrm{kDa}, \mathrm{pI} 10.5)$ was first isolated by Gyorgy et al. in 1941 who investigated the egg-white injury of chicken. [73] It contains four identical subunits forming a dimer of dimers. The monomers bind specifically to biotin with an affinity of $10^{7} \mathrm{M}$. The binding affinity of the tetramer is dramatically higher $\left(10^{15} \mathrm{M}\right)$ and the interaction of avidin and biotin is one of the strongest protein-ligand interactions (binding energy $\approx 35 k_{\mathrm{B}} T$ ). ${ }^{[74]}$ Because of the highly selective and strong binding of avidin to biotin it is often used to mediate interactions based on the key-lock principle. ${ }^{\text {[5] }}$ In this thesis, avidin was used to induce adhesion and immobilization of vesicles to substrate surfaces. 


\subsection{Fluorescence microscopy}

Fluorescence microscopy has been widely used to probe biological samples in an easy, fast and non-invasive manner. A high proportion of the results obtained in the field of cell biology is based on fluorescence. In fluorescence microscopy, the background is reduced due to the specific excitation of fluorophores. The fluorescence emission is shifted to longer wavelengths and is separated from the excitation light which allows to achieve high contrasts. ${ }^{[76]}$ To image samples in three dimensions optical sectioning microscopy is used. By imaging the specimen in different heights a three dimensional image can be reconstructed. Out of focus light is either blocked e.g. by a pinhole (confocal laser scanning microscopy, spinning disc confocal microscopy) or minimized by only exciting the focal plane (multi photon microscopy). Using confocal microscopy slightly higher resolutions are achievable compared to conventional wide-field microscopy.

\subsubsection{Point spread function}

The point spread function (PSF) represents the intensity of a point emitter as a function of the distance to the emitter. It describes how an ideal point emitter would be detected after being transmitted through an objective with a high numerical aperture (NA) and is dependent on the imaging system and the wavelength of the light $\lambda$. Since objectives can not focus light to an infinitesimally small spot, interference occurs which results in a diffraction pattern (airy disc). Concentric rings are imaged in the $x$-y-plane (Figure 3.2. A) and their diameter decreases with increasing $N A$. The diameter of the first minimum of the airy disc is termed airy unit (AU). The geometric pattern of the PSF in $z$-direction is more complex but also determined by an alternating series of intensity minima and maxima resulting from interference (Figure 3.2 B). The lateral and axial intensity profile of the calculated PSF is shown in Figure 3.2 C. The intensity distribution is much smaller for the lateral intensity profile ( $x$ - $y$-plane) than for the axial profile ( $z$-plane) which results in a better lateral than axial resolution. 

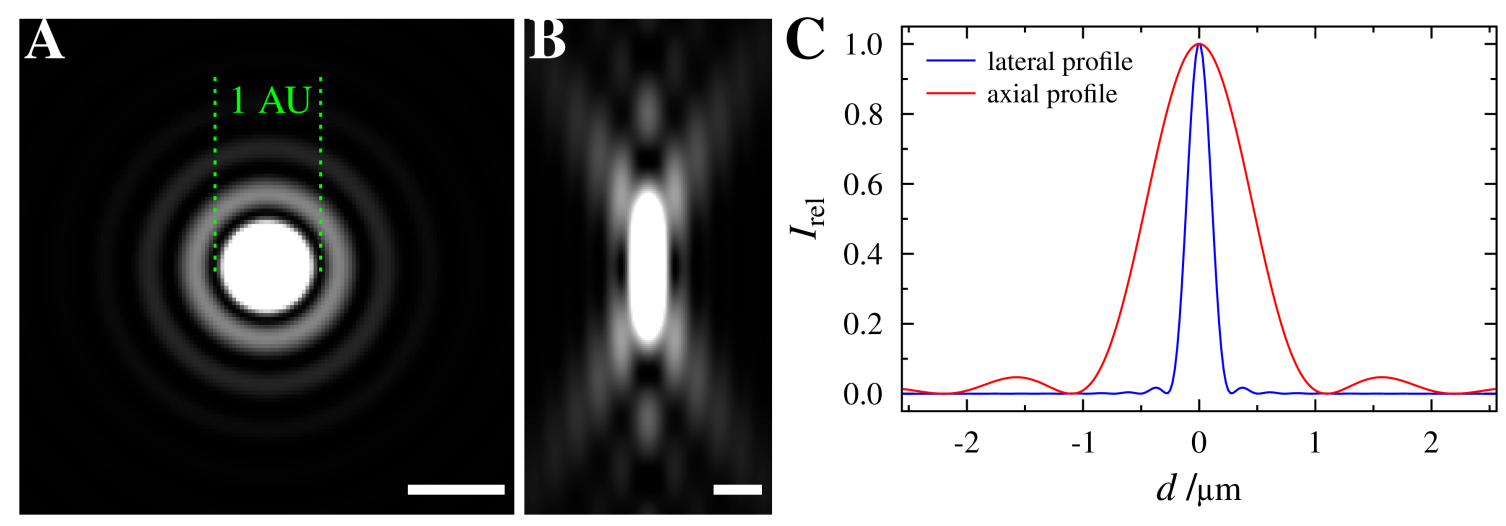

Figure 3.2: Point spread function calculated using the ImageJ plugin PSF Generator and the model of Born and Wolf with $N A=1.1, \lambda=500 \mathrm{~nm}$ and water as immersion medium with a refractive index of $n=1.33 .{ }^{[77+79]}$ A: $x-y$-plane. B: $x$-z-plane. C: Normalized axial and lateral intensity profiles of the calculated PSF. Scale bars: $500 \mathrm{~nm}$.

The resolution of a microscope is the minimal distance required to distinguish two objects. The Rayleigh criterion is the distance between the intensity maxima of two point emitters when the intensity maximum of the PSF of the first emitter is located at the position of the first minimum of the PSF of the second emitter. The intensity between both maxima drops to $73 \%$ of the maximum intensity which allows to distinguish two objects. The width of the PSF is determined by the NA and the wavelength of the emitted light $\lambda_{\mathrm{em}}$. The resolution according to Rayleigh $\left(d_{\text {Rayleigh }}\right)$ is given by:

$$
d_{\text {Rayleigh }}=\frac{0.61 \lambda}{N A} \text {. }
$$

As resolution defined by the Rayleigh criterion is hard to measure, the full width half maxima (FWHM), the width at $50 \%$ of the maximum intensity of a point emitter, is more often used to define the resolution of a microscope. The lateral and axial resolution of a confocal microscope are given by:

$$
d_{\mathrm{FWHM}, \mathrm{xy}}=\frac{0.51 \lambda_{\mathrm{ex}}}{N A}
$$

and

$$
d_{\mathrm{FWHM}, \mathrm{z}}=\frac{0.88 \lambda_{\mathrm{ex}}}{n-\sqrt{n^{2}-N A^{2}}}
$$

with $\lambda_{\text {ex }}$ being the excitation wavelength and $n$ the refractive index of the immersion medium. ${ }^{[80]}$ For visible light the lateral and axial resolution is typically in the range of 200 to $400 \mathrm{~nm}$ and of 0.5 to $2 \mu \mathrm{m}$. 


\subsubsection{Digitalization of an analog signal}

The intensity profile of two adjacent objects with a distance matching the Rayleigh criterion drops to $73.6 \%$ of its intensity between the objects. ${ }^{81}$ Even though the continuity of the PSF results in continuous intensity profiles, recording of intensities involves quantization of the signal. To avoid loss of information the ideal sampling frequency is given by the Nyquist criterion being 2.4 times the highest sample frequency. ${ }^{82}$. To visualize how the sampling frequency changes the detected signal the intensity profile of two objects with a certain distance, approximated as Gaussian functions, was simulated. A low number of four sampling points yields two adjacent sampling points with similar intensity. The two objects appear as one and cannot be distinguished (Figure 3.3 A).
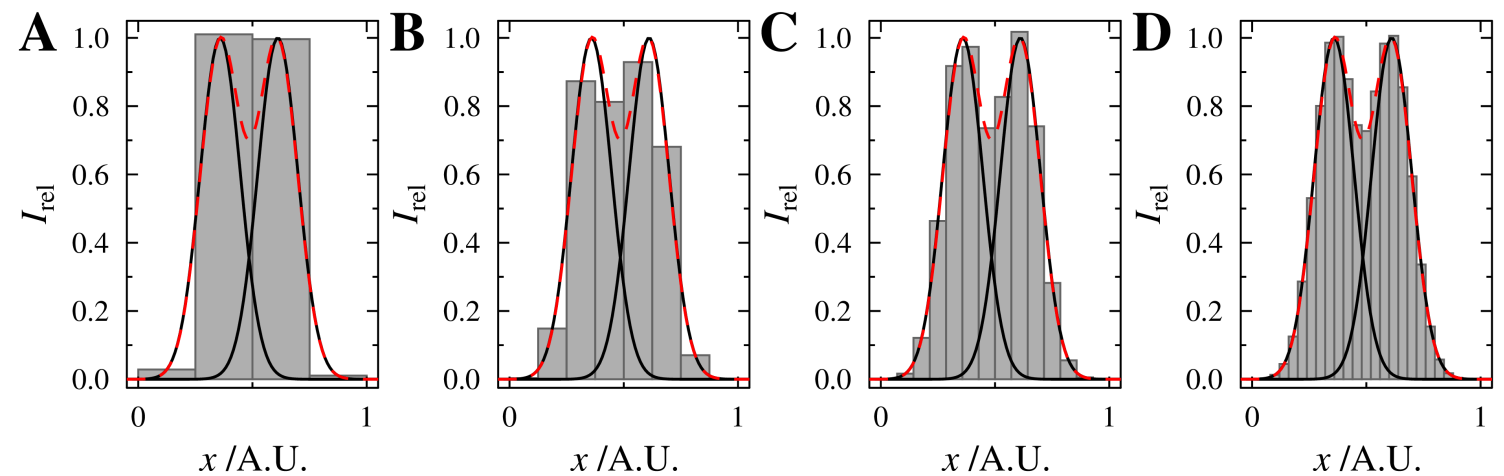

Figure 3.3: Intensity profiles resulting from two objects at a given distance. The intensity profiles are sampled with different sampling rates. A: 4 sampling points: both objects cannot be distinguished and appear as one. B: 8 sampling points: first differences in the intensity profile become visible possibly indicating two objects. C: The intensity between the two objects drops to $73 \%$ and both objects can be clearly distinguished. D: Higher sampling rates allow a finer sampling of the intensity profile.

For a higher sampling rate with eight sampling points first differences in the intensity profile become visible. The intensity difference of the highest sampling point and the sampling point between the two objects is $12 \%$ which is smaller than given by the Rayleigh criterion required to clearly discriminate them (Figure $3.3 \mathrm{~B}$ ). Further increasing the sampling rate results in an intensity difference of $27 \%$ between the maxima allowing to clearly identify two objects (Figure 3.3 C). At higher sampling frequencies more data points allow resolving the intensity profile in more detail but do not yield additional resolution (Figure $3.3 \mathrm{D}$ ). 
Too high sample frequencies can impair image quality as the light dose required for imaging can photo-bleach the fluorophores and decrease the signal-to-noise ratio as for a given photon number each sampling point receives fewer photons. For this reason, a sampling rate up to 2.8 times of the highest sample frequency is often recommend resulting in a light oversampling avoiding loss of information. ${ }^{[82}$ If a high resolution is not essential under-sampling minimizes photo-bleaching and can improve the signal-to-noise ratio and imaging speed.

\subsubsection{Confocal laser scanning microscopy (CLSM)}

Compared to wide-field microscopes which illuminate a large sample area and often detect the fluorescence using a camera, a confocal microscope uses a focused laser beam to illuminate only a small area of the sample. The laser spot is moved point-wise and the image is constructed from the intensities and positions imaged. Out-of-focus light is blocked by a pinhole with adjustable diameter. This allows to adapt the microscope settings to the used objectives, wavelengths and experimental conditions. A schematic setup of a confocal microscope is shown in Figure 3.4. The laser is focused on the sample through the optics of the microscope and the objective illuminating only a small volume. The fluorescence of the excited fluorophores is collected by the objective and passes a dichroic beam splitter. A pinhole with adjustable size in the back focal plane blocks out-of-focus light and only light from the focal plane passes the pinhole and successively the emission filter. A fast scanner is used to move the laser spot on the sample illuminating each position for microseconds (dwell time). Faster frame rates can be achieved using a resonant scanner. Photomultiplier tubes (PMTs) are often used as detectors of fluorescence signals and have a quantum efficiency (QE) of up to 25 to $30 \%$. 82, 83 The signal can be increased by replacing the PMTs with more sensitive GAsP detectors with quantum efficiencies of up to $40 \%$.

The resolution of a confocal microscope can be improved up to a factor of 1.4 using very small pinhole diameters for imaging $(<0.25 \mathrm{AU}) .\left[{ }^{[84}\right]$ The FWHM is then calculated by:

$$
d_{\mathrm{FWHM}, \mathrm{xy}}=\frac{0.37 \bar{\lambda}}{N A}
$$

and

$$
d_{\text {FWHM,z }}=\frac{0.67 \bar{\lambda}}{n-\sqrt{n^{2}-N A^{2}}} .
$$


with $\bar{\lambda} \approx \sqrt{\lambda_{\mathrm{ex}} \cdot \lambda_{\mathrm{em}}}$. The increased resolution originates from almost identical sizes of the PSF of the excitation and emitted light when using small pinhole diameters. ${ }^{85}$ However, an enhancement in resolution of a factor of 1.4 is only achievable for infinitesimal small pinholes. Since the signal-to-noise ratio decreases with decreasing pinhole diameter the usage of small pinholes is unfavorable if not a very high resolution is required. $[84$.

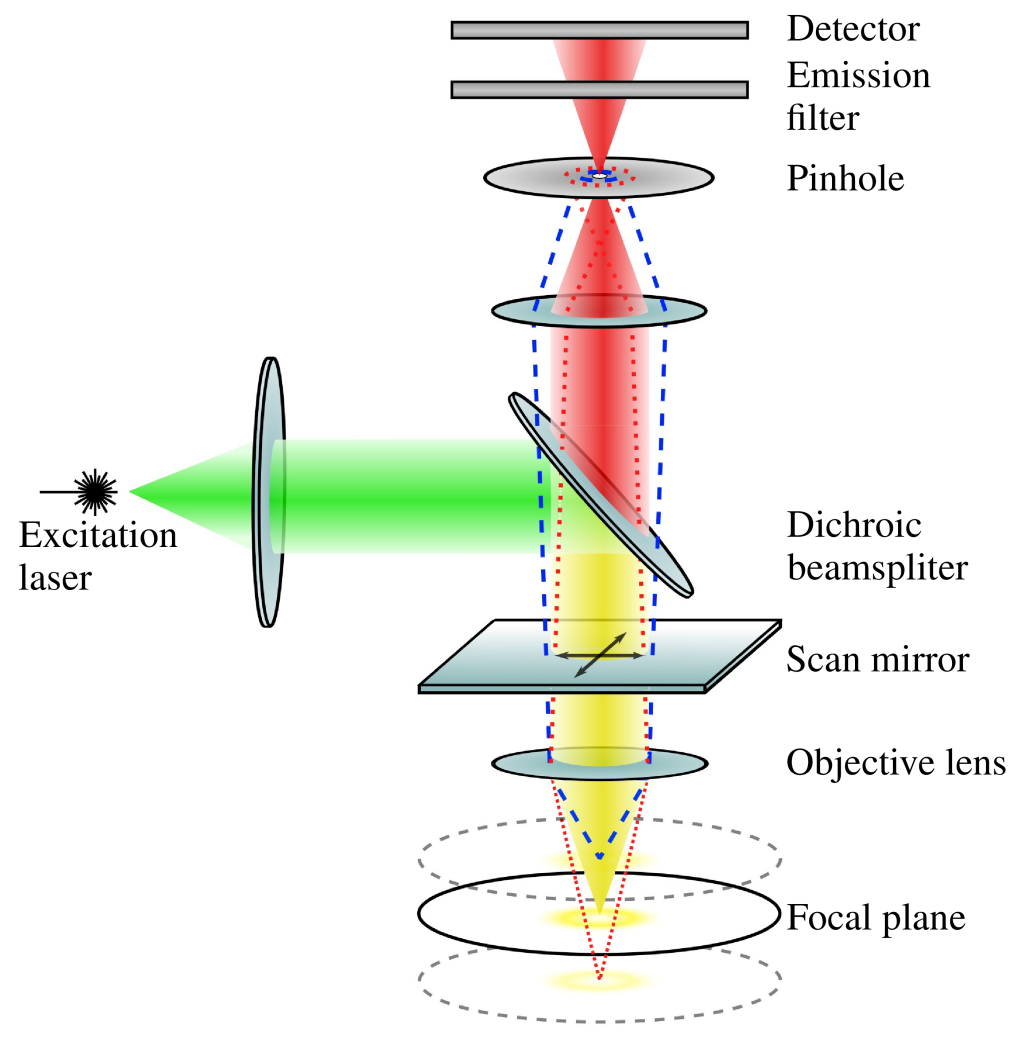

Figure 3.4: Schematic illustration of the operation principle of confocal laser scanning microscopy.

\subsubsection{Spinning disc confocal laser microscopy (SDCLM)}

Rejection of out-of-focus light as introduced by Marvin Minsky in 1955 using a pinhole allows to acquire high quality three dimensional images of samples. Most of the confocal microscopes are single-beam microscopes which scan the specimen point-wise. However, rejection of out-of-focus light is not only limited to a single pinhole. The Nipkow disc has several pinholes spirally arranged allowing 
to confocally image the sample with multiple beams simultaneously, increasing the speed of image acquisition dramatically. ${ }^{[84]}$ It was improved by Yokogawa placing a second disc containing a microlens array over the first one. The setup of the spinning disc microscope used in this thesis is shown in Figure 3.5 .
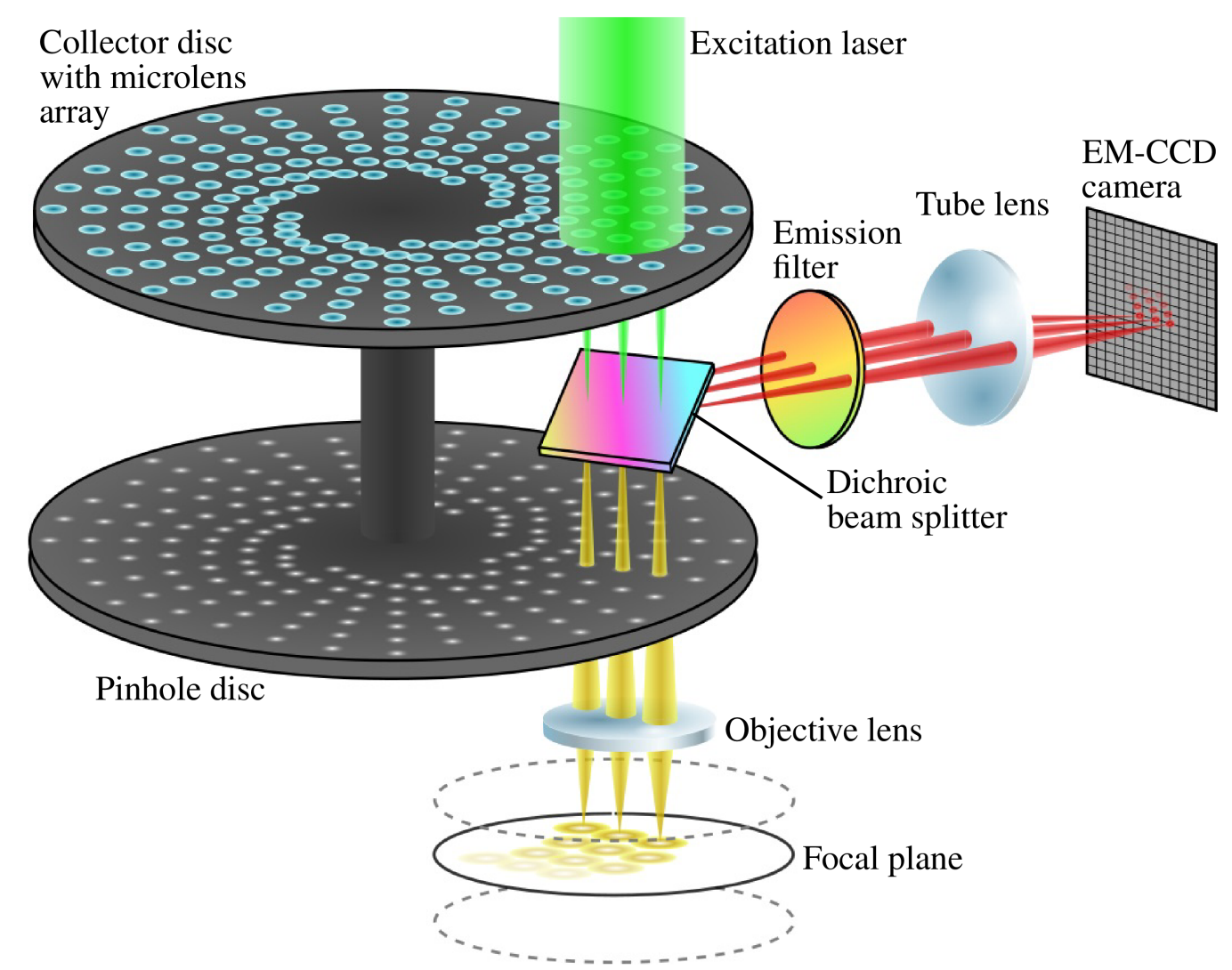

Figure 3.5: Schematic illustration of the operation principle of spinning disc microscopy derived from Toomre et al. .86

The stand of the microscope is custom made (Olympus custom made, Olympus Deutschland GmbH, Hamburg, Germany) allowing simultaneous upright and inverse probing of the sample. Inverse wide field imaging uses a mercury lamp as illumination source and a charged coupled device (CCD) camera as detector. The separation of excitation and emission light is done by filter cubes and a dichroic mirror. Upright illumination using the spinning disc unit uses lasers modulated by an acousto-optic tunable filter (AOTF) as illumination source. This enables to tune the illumination time and intensity fast and precisely. The laser is widened to illuminate about 1000 of 20000 pinholes of the Nipkow disc. [83] The light is then focused by the microlens array in the first disc through the pinholes of the 
second disc and excites the fluorophores of the sample. The fluorescence emission passes the first pinholes and is reflected by a dichroic mirror. An emission filter blocks remaining excitation light and filters the desired range of wavelength for detection. If a simultaneous detection of two fluorophores is necessary, a dichroic mirror and two emission filter can be used to focus emission light of different wavelengths on different regions of the camera. This setup requires only a single camera which ensures an accurate timing of both channels. Back-illuminated electron-multiplying CCD cameras (EMCCDs) are mostly used for detection of the fluorescence in SDCLM because of their high quantum efficiency of more than $90 \%$.

\section{Illumination source}

The microscope is equipped with three lasers (iBeam Smart 405-S, iBeam Smart 488-S, Toptica Photonics AG, Gräfeling, Germany and Cobolt Jive ${ }^{\mathrm{TM}}$ (Cobolt AB, Solna, Sweden) which are modulated in intensity by an AOTF (TF525-250-6-3GH18A, Gooch \& Housego PLC, Ilminster, UK). As the width of the laser beam is typically a few millimeter the laser is widened and collimated to illuminate a larger area of the mircolens array. Therewith, the homogeneity of the illumination of the pinhole disc is also improved. In comparison to CLSM the light dose required to image the same area with identical speed is much lower $\left(1 / 24000^{\text {th }}\right.$ to $1 / 2000^{\text {th }}$ of the intensity dose of a CLSM). $\left.{ }^{83}, 87\right]$ Also excitation of fluorophores is more efficient in SDCLM as the intensity density is lower and the exposure time of each pixel is longer. This avoids saturation of fluorophores where the output of photons does not scale linearly with illumination power. Additionally, bleaching of fluorophores is minimized and photo-toxicity is lowered being especially important for in vivo imaging.

\section{Spinning disc}

The Yokogawa setup of two parallel discs increase the fraction of the illumination light reaching the sample from $1 \%$ up to $60 \%$. The rotation speed of commercially available Yokogawa units is typically $5000 \mathrm{rpm}$ or $10000 \mathrm{rpm}$. Each pinhole is superpositioned after rotation of $30^{\circ}$ allowing to capture 12 images per rotation. This results in a theoretical maximum frame rate of 2000 frames per second. 
However, the actual achievable frame rate is determined by the detector and experimental conditions such as sample brightness and fluorophore stability. When the microlens array is illuminated by the laser it focuses the light through a dichroic beam splitter between the discs through the pinholes of the second disc onto the specimen. In contrast to CLSM, the pinhole aperture in SDCLM is fixed in size. Therefore, the optimal performance and confocality is only given for a single magnification, wavelength and $N A$. The pinhole diameter of $50 \mu \mathrm{m}$ of the Yokogawa unit (Yokogawa CSU-X, Rota Yokogawa GmbH \& Co. KG, Wehr, Germany) used in this thesis, was optimized by the manufacturer for objectives with a magnification $(M F)$ of $60 \times$ and $100 \times$. The best compromise between confocality and intensity is achieved when the pinhole size equals the product of airy disc and magnification. The size of the airy disc is given by twice the Rayleigh resolution (equation 3.28). For light with a wavelength of $500 \mathrm{~nm}$, the used objective (CFI Plan 100XW 100×/1.1, Nikon GmbH, Düsseldorf, Germany) with a magnification of $100 \times$ and a $N A$ of 1.1 this leads to:

$$
d_{\text {pinhole }}=\frac{1.22 \cdot \lambda}{N A} \cdot M F=\frac{1.22 \cdot 0.5 \mu \mathrm{m}}{1.1} \cdot 100=55 \mu \mathrm{m},
$$

which is close to the optimal pinhole diameter of $50 \mu \mathrm{m}$. Objectives having a lower magnification will dramatically reduce the confocality as the projection of the airy disc is smaller than the pinhole.

Even though multiple pinholes reject out-of-focus light efficiently for small imaging depths (Figure 3.6 A,B), pinhole cross talk is an inherent disadvantage of SDCLM. It occurs when fluorophores outside the focal plane are excited. The emission of the fluorophores is then detected by adjacent pinholes which decreases contrast and confocality (Figure 3.6 C). Pinhole cross talk can be reduced by increasing the interpinhole distance or using two-photon excitation. ${ }^{88}$ However, both approaches have disadvantages. The lateral resolution is reduced and fluorophore bleaching is increased in case of the two-photon excitation. The illumination density is decreased in case of an increased inter pinhole distance. To compensate for the reduced excitation, higher illumination intensities would be required which would result in more bleaching of fluorophores. 


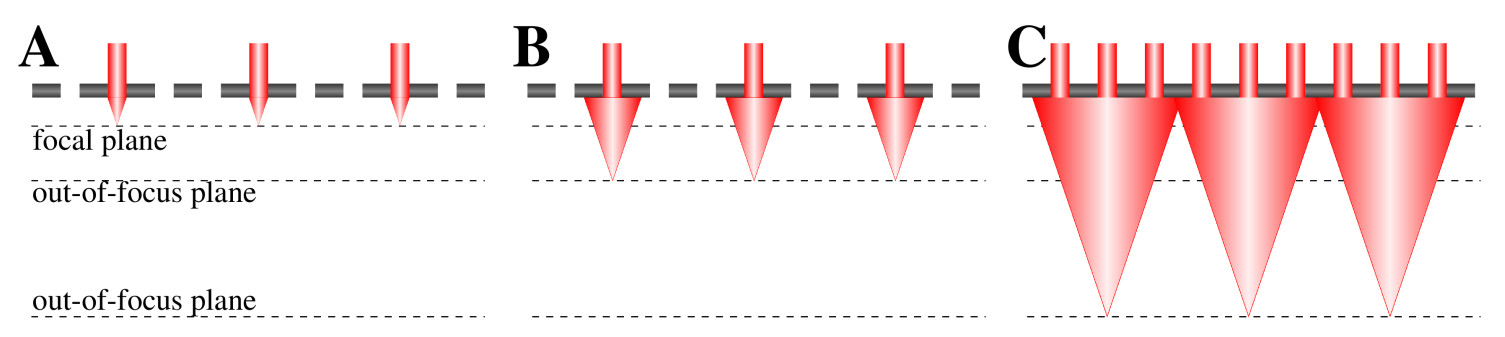

Figure 3.6: Schematic illustration of out-of-focus light resulting in pinhole crosstalk. A: Light from the focal plane passes pinhole. B: Out of focus light near the focal plane is rejected. C: Out-of-focus light far away from the focal plane is detected by adjacent pinholes (pinhole crosstalk).

\section{Emission filters}

After passing the pinholes and the dichroic beam splitter, the fluorescence reaches the emission filter. It is used to block stray light resulting from the laser used for excitation and separates the desired range for detection. An automatic filter wheel (Rotr, Andor Technology Ltd., Belfast, UK) allows fast switching of the emission filters. Typically emission filters have a single or multiple wavelength range when they become transmissive and reflective (cut-on/off wavelengths). Commercially available filters have a sharp cut-on range minimizing fluorophore crosstalk, while reaching transmission efficiencies (TE) better than $95 \%$ resulting in a high fluorescence signal.

Filters can be divided into short-pass, band-pass, long-pass and notch filters. Notch filters are used to block a small range of wavelengths while transmitting the rest. Typically, they are used to block excitation stray light. Short-pass filters have a cut-off wavelength as they block light with longer wavelengths as the cut-off wavelengths. Since the fluorescence emission has a longer wavelength than the excitation light they are not very common in fluorescence microscopy. Bandpass filters transmit light with a narrow range of wavelengths which is required for multi-color imaging. However, they transmit only a fraction of the emission spectrum of a fluorophore therewith decreasing the light intensity compared to a long-pass filter. Long-pass filters transmit light with a longer wavelength than the cut-on wavelength. They can provide the maximum fluorescence intensity and are used when only a single dye is excited or to detect the dye with the highest wavelength with maximum intensity. Transmission spectra of selected emission filters (AHF Analysentechnik AG, Tübingen, Germany) equipped to the 
automatic filter wheel of the spinning disc microscope used in this thesis are shown in Figure 3.7 .
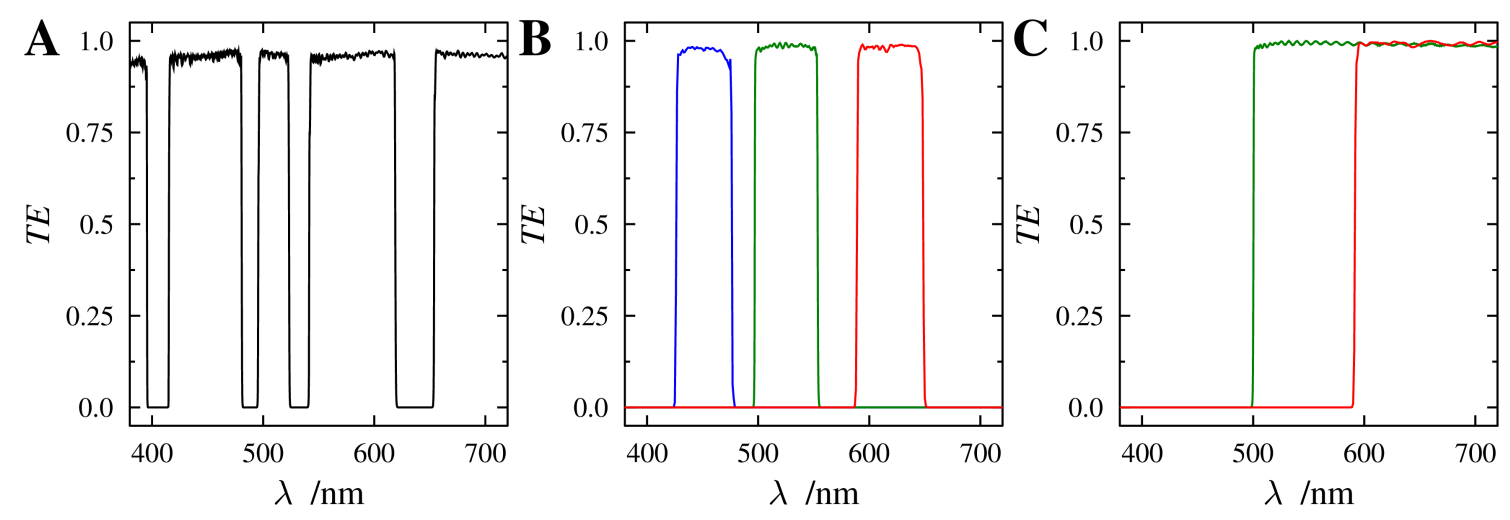

Figure 3.7: Transmission spectra of selected emission filters equipped to the spinning disc microscope. A: Notch filter: QuadLine Rejectionband ZET405/488/561/640. B: Band-pass filters: ET450/50 ET (blue), 525/50 BrightLine HC (green) and ET620/60 ET (green). C: Long-pass filters: 496/LP BrightLine HC (green) and 590 LP ET (red).

A disadvantage of filter based microscopes is the reduced flexibility as for different dye combinations different filters are required. Multi-color experiments require a sequential change of the emission filter which decreases the frame rate as the change of emission filter takes time. Using a filter which only blocks the wavelengths of the lasers used for excitation allows imaging of multiple wavelengths by sequentially switching the excitation laser on and off. Simultaneous detection of multiple dyes is possible by either using two cameras or by focusing the different wavelengths on different regions of the same camera. The latter configuration was equipped for the microscope used in this thesis as it ensures best timing of the signals.

\section{Detector}

Spinning disc microscopes are more sensitive than confocal laser scanning microscopes. ${ }^{84}$. 87] As the overall loss of light inside the optical system of a CLSM and a spinning disc microscope are expected to be similar, differences in efficiency are attributed to the used detectors. ${ }^{[83}$ In CLSM commonly PMTs or GAsP detectors are used which do not preserve spatial information. In SDCLM, cameras are utilized for the detection of the fluorescence. Commercially available PMT and GAsP detectors have a quantum efficiency of 25 to $40 \%$ which is much lower than 
the quantum efficiency of scientific CMOS (sCMOS) and EMCCD cameras (60 to $95 \%$ ). SCMOS cameras have higher readout speeds, larger sensors and a quantum efficiency of typically 60 to $80 \%$. EMCCD cameras have fewer, but larger pixels and a high quantum efficientcy of up to $95 \%$. They multiply the generated electrons in a special register (EM register) by applying a voltage (EM gain, $M$ ) during the readout process. The readout noise $N_{\text {read }}$ decreases proportionally with the applied EM gain which allows to eliminate this source of noise and enables the detection of single photons. However, during the multiplication process statistically electrons are generated leading to an increased noise and decreasing the signal-to-noise ratio $(S N R)$. Additional sources of noise are shot noise $\left(N_{\text {shot }}\right)$, spurious noise $\left(N_{\text {CIC }}\right)$ and thermally induced noise (dark noise, $N_{\text {dark }}$ ). ${ }^{[89]}$ Dark noise is time dependent and can be avoided by cooling the chip to low temperatures. Spurious noise results from the shifting process of the electrons in the pixel register. Since it is a function of the clock voltage during the readout process it is also termed clock induced charged (CIC) being proportional to the number of readouts. Shot noise $\left(N_{\text {shot }}\right)$ results from the particle nature of photons and the Poisson distribution when detecting photons (equation 3.34. ${ }^{82}$ At constant photon flux the amount of photons detected is not constant but statistically distributed. It increases with increasing signal in a square root dependence. Additionally, shot noise is influenced by the quantum efficiency of the detector as the number of electrons generated is proportional to the quantum efficiency (equation 3.34).

$$
N_{\text {shot }}=\sqrt{\text { Signal }}=\sqrt{n_{\text {photon }} \cdot Q E}
$$

The overall noise $N$ is then given by:

$$
N=\sqrt{N_{\text {shot }}^{2}+N_{\text {read }}^{2}+N_{\text {CIC }}^{2}+N_{\text {dark }}^{2}}
$$

Even though CIC determines the actual detection limit of EMCCD cameras for single photon experiments using high EM gains, spurious events are rare and this source of noise can be neglected for higher signals. ${ }^{[89}$ For short acquisition times and low temperatures where also dark noise can be neglected, the $S N R$ is then approximately calculated to:

$$
S N R=\frac{n_{\text {photon }} \cdot Q E}{\sqrt{n_{\text {photon }} \cdot Q E \cdot F_{\mathrm{n}}^{2}+\left(N_{\text {read }} / M\right)^{2}}},
$$


with $F_{\mathrm{n}}^{2}$ being a factor accounting for the additional noise resulting from the amplification process. For sCMOS cameras, $F_{\mathrm{n}}=1$ and $M_{\mathrm{n}}=1$ as no electrons are multiplied. EMCCD cameras efficiently eliminate readout noise by using high multiplication gains $\left(N_{\text {read }} \ll M\right)$ and the additional noise factor was determined to $F_{\mathrm{n}}=\sqrt{2}$. 90 . Theoretical signal-to-noise ratios were calculated as a function of the photon number per pixel for a sCMOS camera assuming $Q E=0.7$ and $N_{\text {read }}=2.3$ and for an EMCCD camera assuming $Q E=0.9$ and neglecting $N_{\text {read }}$ (Figure 3.8. 91, 92]

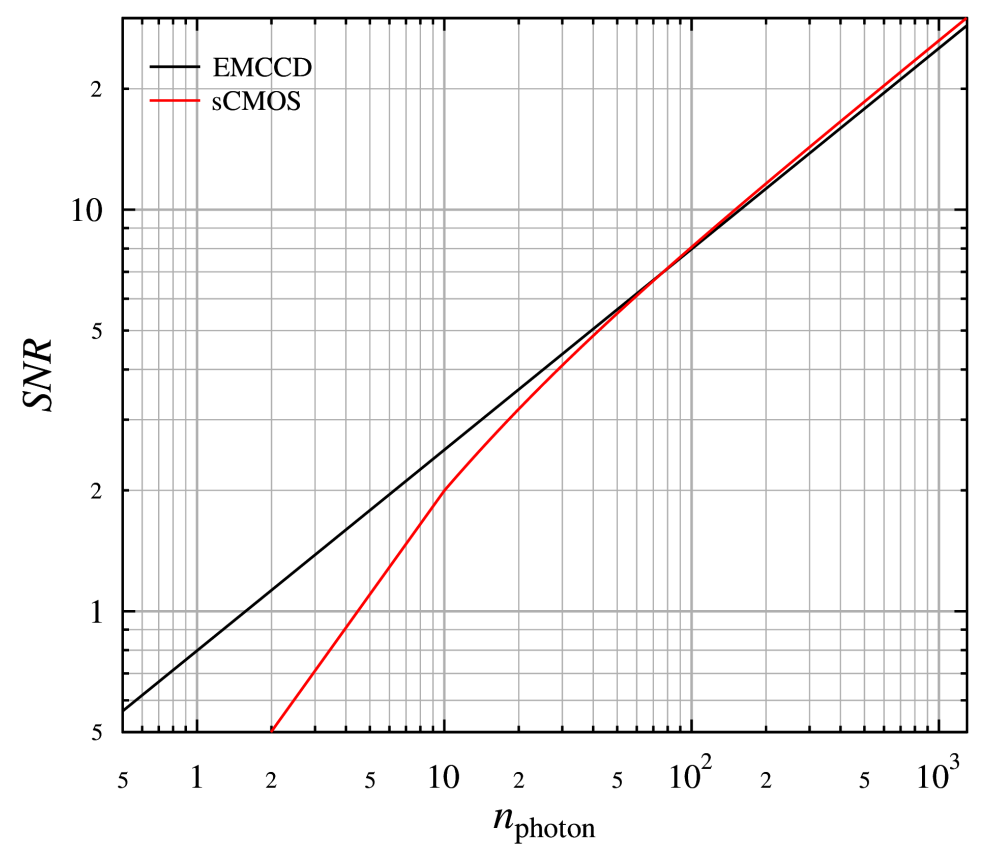

Figure 3.8: Signal-to-noise ratio as a function of the number of photons per pixel.

Based on the described calculation, sCMOS cameras are able to achieve higher a $S N R$ compared to EMCCD cameras at high photon densities ( $>76$ photons per pixel). This threshold can be lowered by using a rolling shutter readout for the sCMOS camera decreasing the readout noise at the cost of a delay between the individual pixel lines which distorts fast moving objects. Also the pixel area of most sCMOS cameras is smaller than those of EMCCD cameras which results in fewer photons per pixel. The conversion of the intensity I measured in counts to incident photons is achieved using equation 3.37 . 


$$
n_{\text {photon }}=\frac{\left(I-I_{\mathrm{dark}}\right) C S}{M \cdot Q E},
$$

with CS being the camera sensitivity, a digital/analog conversion factor, which can be extracted from the camera specifications. Since the photon density in spinning disc microscopy is low as exposure typically lasts only a few milliseconds, EMCCD cameras are best suited for this type of microscope when imaging with very high speed and low light intensities. 93]

The spinning disc microscope used in this thesis is equppid with an EMCCD camera (iXON 897 Ultra, Andor Technology Ltd., Belfast, UK) which has a maximum frame rate of 56 frames per second (fps) at full resolution $(512 \times 512$ pixels). The pixel size of $16 \mu \mathrm{m}$ results in $133 \mathrm{~nm}$ per pixel when imaging with an objective having a magnification of $100 \times$ and using an additional lens with a magnification of 1.2. As this pixel size is close to the Nyquist criterion, it provides the optimal compromise between resolution and sensitivity.

Compared to PMTs, CCD cameras also have a higher dynamic range $(D R)$ which is defined as the quotient of the maximum electron capacity of a camera pixel (full well capacity, $F W C$ ) to the detection limit $(D L)$ which allows measuring dim and bright objects simultaneously (equation 3.38.

$$
D R=\frac{F W C}{D L}
$$

As for EMCCD cameras the electrons are amplified in the EM register, the full well capacity of a pixel is replaced by the full well capacity of the EM gain register. The detection limit is given by the readout noise which decreases as a function of the applied EM gain. [90] The readout noise increases with the square root of readout frequency. ${ }^{82]}$ Even though EMCCD cameras are able to eliminate readout noise at high EM gains it must be considered as $\geq 1$ electron when calculating the dynamic range. As the readout noise decreases with increasing EM gain and the FWC of the EM register is larger than the FWC of the pixels the dynamic range increases reaching a plateau where the decrease of the readout noise is compensated by the decreased full well capacity of the EM gain register. After application of EM gain the detection limit decreases to one electron and the dynamic range decreases. The dynamic range of the EMCDD camera used in this thesis as a function of the applied EM gain is shown in Figure 3.9 for different readout frequencies. 


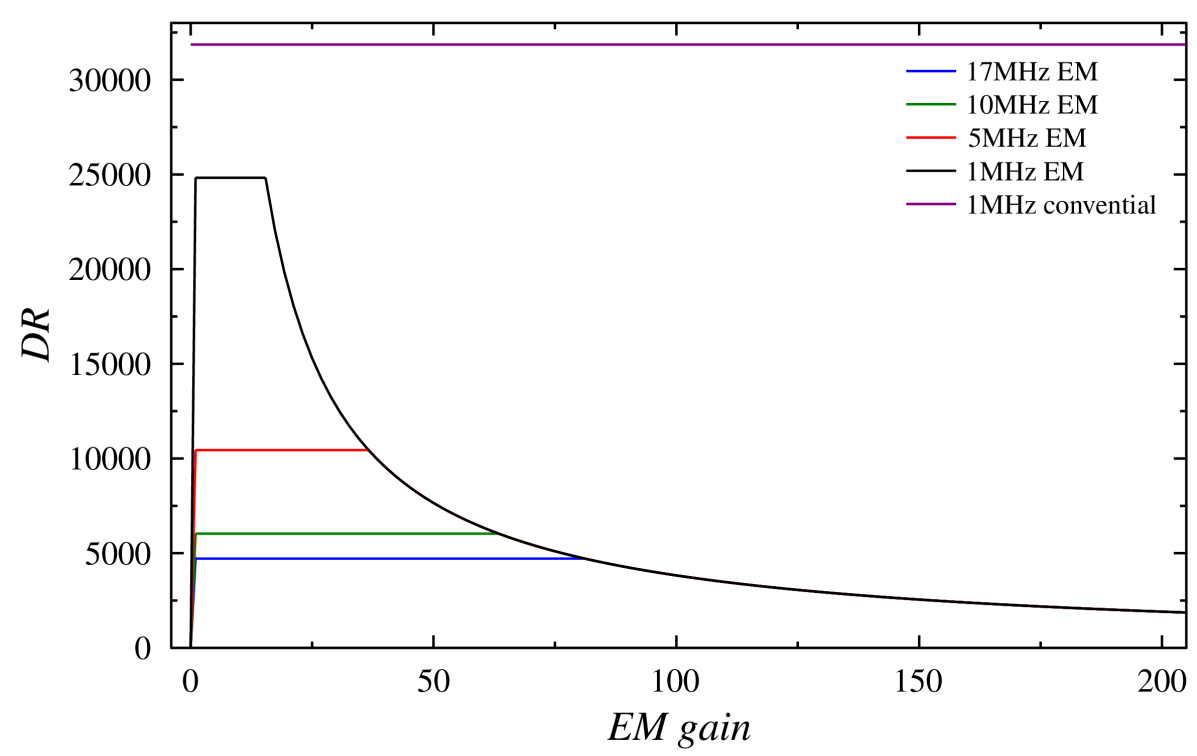

Figure 3.9: Dynamic range as a function of the applied EM gain for different readout speeds.

Even though the theoretical speed of the spinning disc microscope is limited by the rotation speed of the Nipkow disc, the actual speed is mostly limited by the brightness of the sample and the speed of the camera. The maximum frame rate of the camera is a function of the exposure and readout time. EMCCD cameras often use frame-transfer CCDs which have an additional area where the image is shifted and stored for the readout and amplification process. This allows to minimize the delay resulting from image readout by detecting the next frame when reading out the current. For exposure times larger than the readout time, the frame rate is only determined by the exposure time. However, to avoid artifacts this requires a perfect synchronization of camera, laser illumination and rotation speed of the Nipkow disc. 94 ] 


\subsection{Experimental methods}

\subsubsection{Preparation of unilamellar vesicles}

\section{Small and large unilamellar vesicles}

Lipid films ( $c=0.4$ to $1 \mathrm{mg}$ ) were prepared from stock solutions of $(c=1$ to $10 \mathrm{mg} / \mathrm{mL}$ ) of the lipids dissolved in $\mathrm{CHCl}_{3}$. PIP 2 was dissolved in $\mathrm{CHCl}_{3} / \mathrm{MeOH} / \mathrm{H}_{2} \mathrm{O}$ (20:9:1). Mixtures of desired composition were prepared in glass tubes. For $\mathrm{PIP}_{2}$-containing lipid mixtures a mixture of $\mathrm{CHCl}_{3} / \mathrm{MeOH}$ (96:4) was used as solvent. The solvent was then removed by using a stream of nitrogen and the films were further dried inside a vacuum oven for a minimum of $3 \mathrm{~h}$. The tubes were sealed with Parafilm ${ }^{\circledR}$ and stored at $4{ }^{\circ} \mathrm{C}$.

The films were rehydrated for $30 \mathrm{~min}$ in buffer and then vortexed three times for $30 \mathrm{~s}$ in an interval of $5 \mathrm{~min}$ to form multilamellar vesicles. Small unilmellar vesicles (SUVs) and large unilamellar vesicles (LUVs) were prepared from the rehydrated lipid films. For reflectometric interference spectroscopy experiments citrate buffer ( $50 \mathrm{~mm} \mathrm{KCl}, 20 \mathrm{~mm}$ Na-citrate, $0.1 \mathrm{~mm}$ EDTA, $0.1 \mathrm{~mm} \mathrm{NaN}_{3}, \mathrm{pH} 4.8$ ) was used for rehydration of the lipid films containing PIP 2 . PBS buffer (PBS, phosphate buffered saline, $136 \mathrm{~mm} \mathrm{NaCl}, 2.7 \mathrm{~mm} \mathrm{KCl}, 1.5 \mathrm{mM} \mathrm{KH}_{2} \mathrm{PO}_{4}, 8.1 \mathrm{~mm} \mathrm{Na}{ }_{2} \mathrm{HPO}_{4}$, $\mathrm{pH} 7.4,280 \mathrm{mOsmol} / \mathrm{L}$ ) was used to rehydrate lipid films lacking $\mathrm{PIP}_{2}$ for the formation of SUVs for reflectometric interference spectroscopy experiments and for rehydration of lipid films for the preparation of LUVs with and without $\mathrm{PIP}_{2}$ for surface plasmon resonance spectroscopy experiments.

From the multilamellar vesicles small unilamellar vesicles (SUVs) with diameters ranging from 20 to $50 \mathrm{~nm}$ were prepared by sonification using a Bandelin sonopuls bath sonifier $(2 \times 15 \mathrm{~min}$, 4 clycles, 60 to $70 \%$ intensity). The water was exchanged between the sonification steps to reduce water heating. Large unilamellar vesicles were prepared by extrusion of the multilamellar vesicles through a polycarbonate membrane with pore diameters of $100 \mathrm{~nm}$ using a miniextruder.

\section{Giant unilamellar vesicles}

Giant unilamellar vesicles (GUVs) became an important model system for cell membranes as they are easy to produce and large enough in size to be imaged by 
optical microscopy. The vesicle membrane separates the interior from the external buffer and protein-mediated transport of molecules has been analyzed as well as fusion and fission of vesicles. The membrane composition and mechanical properties can be easily controlled which allows to study processes as a function of membrane composition and e.g. membrane tension. 95]

The electro-formation technique described by Angelova et al. was used for the preparation of giant unilamellar vesicles (GUVs). ${ }^{[96}$ Onto indium tin oxide-coated glass slides (ITOs, Präzisions Glas \& Optik GmbH, Iserlohn, Germany) 25 to $50 \mu \mathrm{L}$ of lipid films ( $c=0.8$ to $1.6 \mathrm{mg} / \mathrm{mL}$ ) with desired lipid composition were transferred and evenly distributed. After mounting of two ITOs, sucrose solution $(264 \mathrm{mOsmol} / \mathrm{L})$ was added to a chamber sealed by a Teflon ${ }^{\circledR}$ ring and the ITOs. An external electric AC field ( $V_{p p}=3 \mathrm{~V}, f=5 \mathrm{~Hz}$ ) was applied for $2 \mathrm{~h}$. The GUVs were then transferred into an Eppendorf ${ }^{\circledR}$ cup and stored at room temperature for maximum five days.

\subsubsection{Preparation of pore-spanning membranes}

Porous silicon substrates (E800, Nanospot GmbH, Münster, Germany) with pore diameters of $850 \mathrm{~nm}$, pore depths of $10 \mu \mathrm{m}$ and a porosity of $2 \%$ was cleaned by a Ar-plasma (60 s, $p=0.015$ bar, $P=60 \%$ ) using an Zepto plasma cleaner (Diener Electronic, Ebbhausen, Germany). The substrates were then glued to a petri dish, buffer was added and potentially entrapped air inside the pores was removed by sonification (3 s). GUVs $(50 \mu \mathrm{L})$ were sedimented in $1.7 \mathrm{~mL}$ of $\mathrm{KCl}$ buffer $(160 \mathrm{~mm}$ $\mathrm{KCl}, 10 \mathrm{~mm}$ MOPS, pH 7.4, $323 \mathrm{mOsmol} / \mathrm{L}$ ) or PBS and then added to the substrate. Pore-spanning membranes were formed by spreading of GUVs (30 min). Nonspread vesicles adhering on the substrate were removed by rinsing.

The protective varnish of porous silicon and silicon dioxide substrates (center of advanced european studies and research (caesar), Bonn, Germany) with a pore diameter of $3.5 \mu \mathrm{m}$ or $4.5 \mu \mathrm{m}$, a pore depth of $8 \mu \mathrm{m}$ and a porosity of $10 \%$ or $20 \%$ was removed in boiling acetone $\left(43^{\circ} \mathrm{C}, 5 \mathrm{~min}\right)$. The substrates were then cleaned by applying an argon and consecutively an oxygen plasma for $30 \mathrm{~s}$ ( $p=0.015 \mathrm{bar}$, $P=60 \%$ ). Titanium was sputtered onto the substrate surface using a sputter coater 108auto (Cressington, UK, $30 \mathrm{~s}, 40 \mathrm{~mA}, 0.06 \mathrm{mbar}$ ) serving as crystallization nuclei for further coating with gold. Thermal evaporation was used to coat the substrates with $30 \mathrm{~nm}$ of gold using a MED020 coating system (Bal-Tec, Germany, 
$p=5 \cdot 10^{-6}$ mbar). Incubation of substrates in 6-mercaptohexane-1-ol (10 mm in $n$-propane-1-ol, over night) led to the formation of a self-assembled monolayer. $10 \mu \mathrm{L}$ of $\mathrm{EtOH}$ were added to the hydrophilically functionalized substrates to remove air trapped inside the pores. Successively, PBS was added and EtOH was removed by carefull rinsing. To $1.8 \mathrm{~mL}$ of PBS $20 \mu \mathrm{L}$ of the GUVs were added and sedimented. $50 \mu \mathrm{L}$ of the sedimented GUVs were then added to the substrates. The PBS buffer used for atmic force microscopy experiments contained $2 \mathrm{~mm}$ of the water soluble dye pyranine which was used for localization of membrane covered pores using fluorescence microscopy. Pore-spanning membranes were formed by spreading of the GUVs and non-spread vesicles were removed by rinsing with buffer after $30 \mathrm{~min}$.

\subsubsection{Immobilization of biotinylated giant unilamellar vesicles onto an avidin coated glass slide}

Glass slides (D 263 M Schott glass 25 mm x 75 mm, ibidi GmbH, Munich, Germany) were treated with a mixture of $\mathrm{H}_{2} \mathrm{O} / \mathrm{NH}_{3} / \mathrm{H}_{2} \mathrm{O}_{2},(5: 1: 1, v / v / v)$ for 20 min at $70^{\circ} \mathrm{C}$. The glass slides were then glued to a petri dish avoiding drying of the substrate to maintain the hydrophilicity. After incubation with avidin $(500 \mu \mathrm{L}, 10 \mu \mathrm{M}$ in PBS) for 30 to 60 min the buffer was exchanged by carefull rinsing with buffer containg bovine serum albumine (BSA, $1 \mathrm{mg} / \mathrm{mL}$ in PBS). By incubation with BSA, hydrophobic regions are blocked and unspecific binding of ENTH to the substrate is minimized. After 30 to $60 \mathrm{~min}$ the BSA-containing buffer was exchanged by careful rinsing with PBS. PBS was replaced by sucrose buffer (sucrose, 2 mM HEPES,

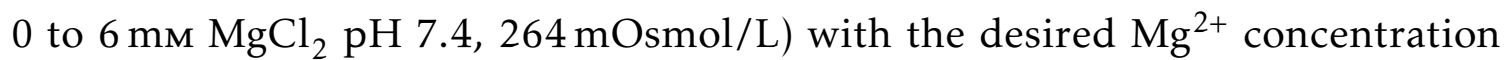
immediately before the experiment in order to avoid changes in osmolarity due to evaporation of water.

\subsection{Biosensing techniques}

Biosensing techniques have become powerful tools for the detection of small quantities of analyte. A main advantage is their high sensitivity allowing the detection up to femtomolar concentrations of analyte and the labeling-free operation 
principle. 97 This allows to measure binding of molecules which are not influenced by an attached label possibly altering their function or binding affinity. A large number of biosensing techniques measures the refractive index which can be correlated to a sample concentration or a surface density. In this thesis, surface plasmon resonance spectroscopy and reflectometric interference spectroscopy were used to monitor binding of ENTH to artificial lipid bilayers.

\subsubsection{Surface plasmon resonance spectroscopy}

Surface plasmon resonance (SPR) spectroscopy is an optical, labeling-free technique allowing the determination of changes in thickness of an adsorbed layer at the water/metal interface. Surface plasmons are charge density oscillations occurring at the interface of two media with dielectric constants of opposite signs (Figure 3.10 A). ${ }^{97}$ Surface plasmons of a noble metal can be excited by an evanescent field using total internal reflection and decay exponentially. ${ }^{[98]}$ Light from a medium with a dielectric constant $\epsilon_{1}$ enters a high refractive prism with a dielectric constant $\epsilon_{2}$ and is refracted (Figure $3.10 \mathrm{~B}$ ).
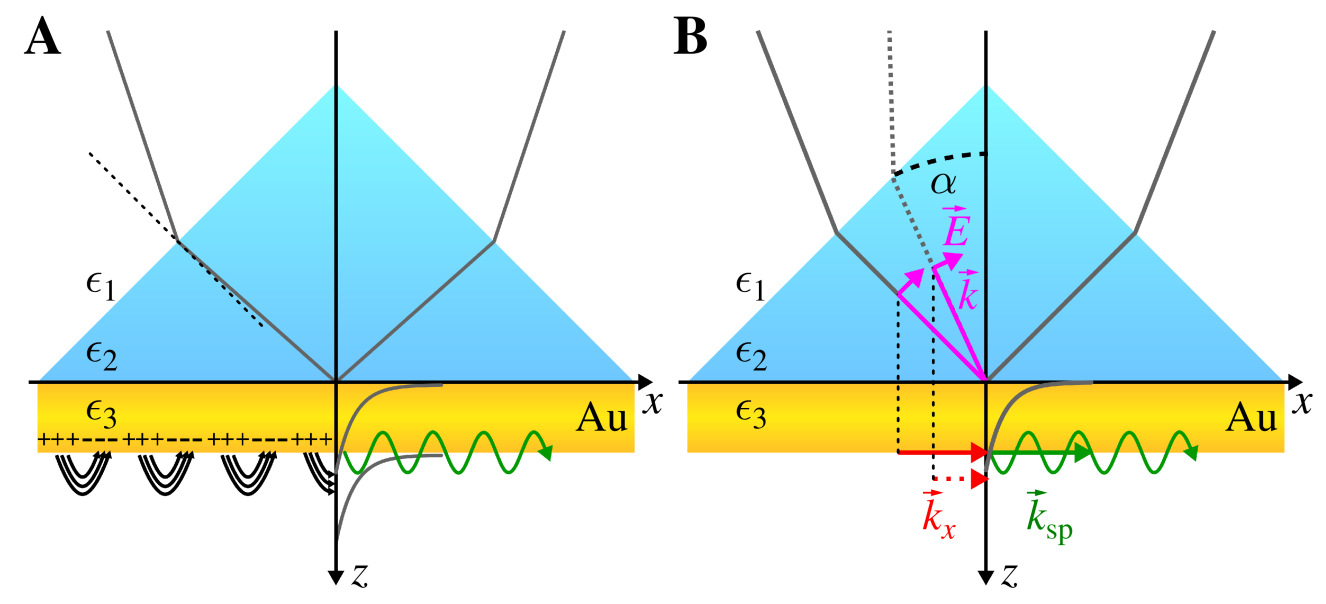

Figure 3.10: A: Charge density oscillations are excited by an evanescent field after total internal reflection. B: The $x$-component of the momentum $k_{x}$ of p-polarized light (wave vector $\vec{E}$ ) increases with increasing angle of incident $\alpha$. Surface plasmons are excited when the momentum of the light matches the momentum of the surface plasmons $\left(k_{\mathrm{sp}}\right)$. Dielectric constants correspond to: $\epsilon_{1}=$ air, $\epsilon_{2}=$ prism, $\epsilon_{3}=$ gold.

The light travels through the prism which is directly attached to a gold layer with a dielectric constant $\epsilon_{3}$ (Kretschmann configuration) and is reflected at the gold layer. 
Depending on the angle $\alpha$ it can excite surface plasmons. As surface plasmons only propagate in $x$-direction, only the $x$-component of the photons momentum $\left(\vec{k}_{x}=\vec{k} \cdot \sin \alpha\right)$ is responsible for the excitation. The momentum of the surface plasmon $\vec{k}_{\mathrm{sp}}$ as a function of the dielectric constants is given by:

$$
\vec{k}_{\mathrm{sp}}=\frac{\omega}{c} \sqrt{\frac{\epsilon_{2} \epsilon_{3}}{\epsilon_{2}+\epsilon_{3}}},
$$

with $\omega$ being the angular frequency. ${ }^{[99]}$ The $x$-component of the momentum of a photon traveling in a medium with a dielectric constant of $\epsilon_{1}$ is given by:

$$
\vec{k}_{x}=\frac{\omega}{c} \sqrt{\epsilon_{1}} \sin \alpha
$$

As the momentum of the surface plasmons is larger than those of photons traveling through air, coupling to a prism with a high refractive index is essential to excite the surface plasmons. The condition for excitation of the plasmons is then given by:

$$
\vec{k}_{x}=\frac{\omega}{c} \sqrt{\epsilon_{2}} \sin \alpha=\vec{k}_{\mathrm{sp}}
$$

It can be seen that the excitation efficiency is a function of the incident angle of the light (Figure 3.11 A). Changes of the refractive index will shift the angle of maximum excitation to higher values. Therefore, at a constant angle $\alpha$ the changes in the refractive index caused by adsorption of molecules will increase the reflectivity $R$ which can be measured as a function of time $t$ (Figure $3.11 \mathrm{~B}$ ).
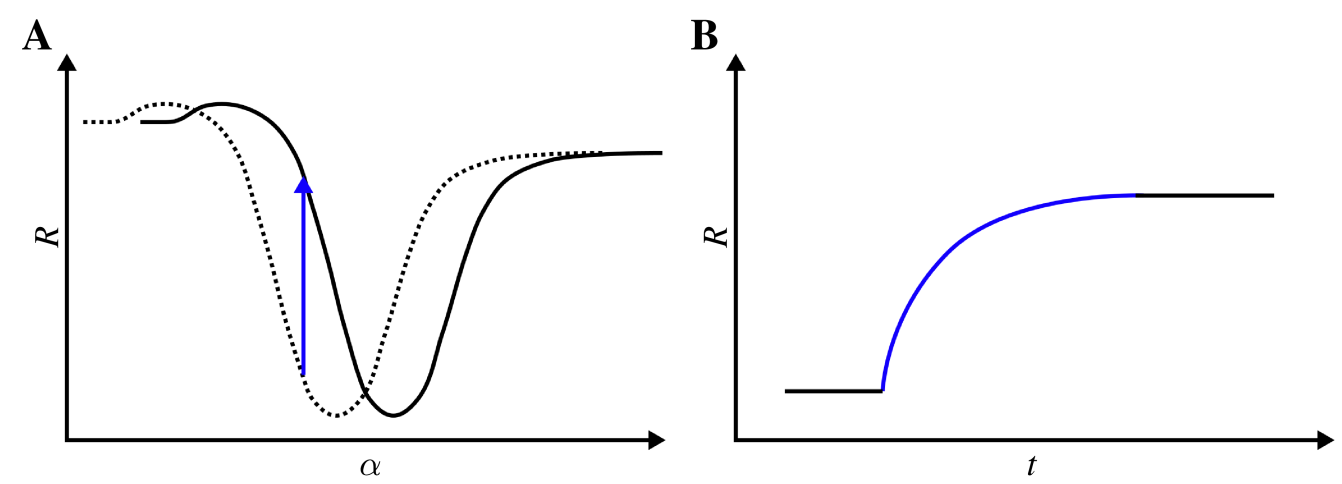

Figure 3.11: A: Schematic illustration of the reflectivity $R$ as a function of the angle $\alpha$. The excitation of the surface plasmon shifts to higher contact angles as a result of the adsorption. B: Schematic illustration of the measured reflectivity at a constant angle as a function of time $t$. 


\section{Experimental procedure}

The device (Reichert SR700DC, New York, USA) was cleaned with a solution of $0.5 \%$ sodium dodecyl sulfate (SDS) and $50 \mathrm{~mm}$ glycerin for 1 to $2 \mathrm{~h}$. After rinsing with PBS, SPR chips (CMD500I sensorchip, XanTec bioanalytics GmbH, Düsseldorf, Germany) with a carboxymethyl hydrogel were inserted in the SPR device and incubated with N-hydroxysuccinimide (NHS, $c=10 \mathrm{mg} / \mathrm{mL}$ ) in MES buffer (50 mм 2-(N-morpholino)ethanesulfonic acid, pH 5.0). 1-ethyl-3(3-dimethylaminopropyl)carbodiimide (EDC, $40 \mathrm{~mm}$ ) was added to activate the carboxyl groups of the hydrogel. After rinsing with PBS, the supernatant of a mixture of $1 \mathrm{~mL}$ phytosphingosine in DMSO $(1 \mathrm{mg} / \mathrm{mL})$ and $1 \mathrm{~mL}$ sodium acetate ( $20 \mathrm{~mm}, \mathrm{pH}$ 5.2) was added to the SPR chip for $45 \mathrm{~min}$. The amine group of phytosphingosine nucleophilically attacks the activated caboxyl group of the carboxymethyl hydrogel leading to a phytosphingosine functionalized hydrogel. The SPR chip was then incubated with ethanolamine hydrochloride ( $1 \mathrm{M}, \mathrm{pH} 8.5)$ for $10 \mathrm{~min}$. After rinsing with PBS, the system was ready for measuring.

LUVs of desired composition were prepared as described in Section 3.5.1. The vesicle suspension $(500 \mu \mathrm{L})$ was diluted with $5.5 \mathrm{~mL}$ PBS and then slowly added to the measurement channel for $1 \mathrm{~h}$. The hydrophobic chain of phytosphingosine intercalates into the lipid bilayer therewith immobilizing the vesicles to the hydrogel. After rinsing with PBS, vesicles lacking the receptor lipid were slowly added to measurement and reference channel for $1 \mathrm{~h}$. Reversibly bound vesicles were removed by rinsing with PBS followed by successive rinsing with $\mathrm{NaOH}(5 \times 2 \mathrm{~mL}$, $50 \mathrm{~mm}$ ). Solutions with different concentrations of ENTH were added to the system after rinsing with PBS. Each addition of protein took about $20 \mathrm{~min}$ and was followed by rinsing with PBS until a plateau was reached. Protein concentrations were increased until saturation of the receptors with protein was observed. The different steps of a typical SPR spectroscopy experiment are summarized in Table 3.2. 
Table 3.2: Flow rates and durations of the different steps of a SPR spectroscopy experiment.

\begin{tabular}{rcl}
\hline step & flow rate $/ \mu \mathrm{L} / \mathrm{min}$ & $t / \mathrm{min}$ \\
\hline SDS, glycine & 50 & $60-120$ \\
PBS & 50 & 20 \\
NHS & 20 & 20 \\
EDC & 20 & 20 \\
PBS & 20 & 20 \\
phytosphingosine & 10 & 45 \\
ethanolamine hydrochloride & 25 & 10 \\
PBS & 25 & 20 \\
vesicles & 50 & 60 \\
reference vesicles & 50 & 60 \\
NaOH & 20 & $5 \times 2$ \\
PBS & 25 & 20 \\
ENTH & 20 & 20 \\
PBS & 25 & $20-30$
\end{tabular}

\subsubsection{Reflectometric interference spectroscopy}

Reflectometric interference spectroscopy (RIfS) is a biosensing technique using interference of white light to measure optical thicknesses. Light traveling in a medium with a refractive index $n_{1}$ is refracted and reflected when hitting an optically transparent medium with a refractive index $n_{2}$ at the angle $\alpha_{1}$ (Figure 3.12).

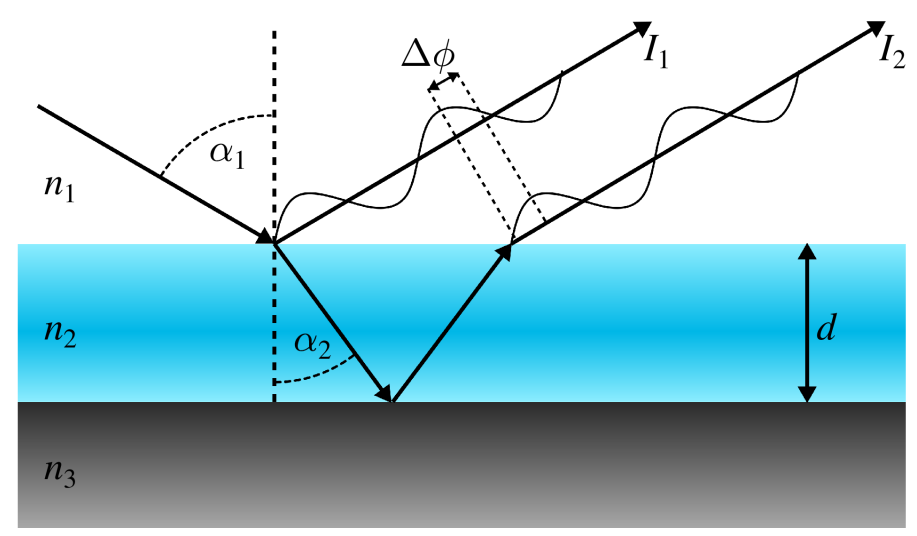

Figure 3.12: Schematic illustration of the light pathway in RIfS. 
As the second medium has a higher refractive index than the first one, light hitting the second medium at the angle $\alpha_{1}$ is refracted towards the normal at the angle $\alpha_{2}$. After being reflected at an optically intransparent medium with a refractive index $n_{3}$, it is refracted again at the interface of the first and second medium. The additional traveling distance $s$ of the refracted light $I_{2}$ causes a phase shift $\Delta \phi$ to the originally reflected light $I_{1} \cdot{ }^{[100]}$ As the optical distance is the product of refractive index and physical distance (equation 3.42),

$$
s_{\mathrm{OT}}=n \cdot s
$$

the optical distance of the refracted light is given by:

$$
s_{\mathrm{OT}}=\frac{2 n_{2} d}{\cos \alpha_{2}}
$$

with $d$ being the physical thickness of the optically transparent layer. For $\alpha=\alpha_{1}=$ $\alpha_{2}=0$ equation 3.43 simplifies to:

$$
s_{\mathrm{OT}}=2 n_{2} d
$$

Depending on the phase shift, the light interferes constructively and destructively. Constructive interference occurs if $\Delta \phi$ is a multiple of the wavelength. For perpendicular shone light it is given by:

$$
\Delta \phi=2 \pi \frac{O T}{\lambda}
$$

with OT being the optical thickness (equation 3.46).

$$
O T=n \cdot d
$$

The interference pattern depends on the wavelength, the refractive index of the optically transparent medium and its physical thickness. For interference the coherence length of the light must be larger than the optical path difference. For white light, a physical thickness of 0.5 to $10 \mu \mathrm{m}$ is required to result in interference. ${ }^{101}$ For $\alpha=0$, the reflectivity $R$ is calculated to:

$$
R=\left|r_{123}\right|^{2}=\frac{r_{12}^{2}+r_{23}^{2}+2 r_{12} r_{23} \cos \left(\frac{4 \pi}{\lambda} O T\right)}{1+r_{12}^{2} r_{23}^{2}+2 r_{12} r_{23} \cos \left(\frac{4 \pi}{\lambda} O T\right)},
$$


with $r$ being the Fresnel reflection coefficient defined as: $r_{i j}=\left(n_{i}-n_{j}\right) /\left(n_{i}+n_{j}\right)$.

\section{Experimental procedure}

Silicon wafers $\left(\approx 2 \times 2 \mathrm{~cm}, n_{3}=3.84^{[102]}\right)$ with an oxide layer of $5 \mu \mathrm{m}$ were incubated in a mixture of $\mathrm{H}_{2} \mathrm{O} / \mathrm{NH}_{3} / \mathrm{H}_{2} \mathrm{O}_{2},(5: 1: 1, v / v / v)$ for $20 \mathrm{~min}$ at $70^{\circ} \mathrm{C}$. The hydrophilic wafers were stored in water and could be used up to two days. Prior to the experiment, the wafers were rinsed with $\mathrm{EtOH}$ and $\mathrm{H}_{2} \mathrm{O}$, dried with a $\mathrm{N}_{2}$ stream and then clamped between an aluminum and an acryl glass block. The refractive index of the $\mathrm{SiO}_{2}$ layer depends on the wavelength $\left(n_{2}(\lambda)=1.47\right.$ to $1.45^{[103}$, $\lambda=400$ to $800 \mathrm{~nm}$ ). An area of about $30 \mathrm{~mm}^{2}$ of the silcon wafer was sealed by a rubber band. Aqueous solutions $\left(n_{1}=1.33^{[104]}\right)$ were transferred through the chamber by a peristaltic pump (flow rate: $\approx 470 \mu \mathrm{L} / \mathrm{min}$ ). An area of $1 \mathrm{~mm}^{2}$ of the wafer surface was illuminated by a tungsten lamp and the intensity spectra were recorded using a NanoCalc-2000 vis/NIR spectrometer (Ocean Optics, Dunedin, USA) or a SD2000 spectrometer (Ocean Optics, Dunedin, USA). The reflectivity was calculated from the measured intensity spectrum $I_{\mathrm{m}}$ according to:

$$
R=\frac{I_{\mathrm{m}}-I_{\mathrm{d}}}{I_{\mathrm{r}}-I_{\mathrm{d}}}
$$

with $I_{\mathrm{d}}$ and $I_{\mathrm{r}}$ being a dark and a reference spectrum. For the reference spectrum an electro-polished aluminum surface was illuminated and the dark spectrum was recorded without illumination. Equation 3.47 was fitted to the data to obtain the optical thickness which was plotted as a function of time.

Addition of small unilamellar vesicles of desired composition led to the formation of a lipid bilayer. Citrate buffer with $\mathrm{pH} 4.8$ was required to form bilayers containing $10 \% \mathrm{PIP}_{2}$. The negative charges of the $\mathrm{SiO}_{2}$ surface and $\mathrm{PIP}_{2}$ are reduced at low $\mathrm{pH}$ values enabling spreading of vesicles. [105] After bilayer formation the buffer was exchanged to PBS. BSA $(c=1 \mathrm{mg} / \mathrm{mL})$ was flushed through the system to block eventual hydrophobic defects to which ENTH could unspecifically bind. After exchange of the BSA-containing buffer to PBS, ENTH was added. Specific binding of ENTH to its receptor lipid increased the optical thickness. After a plateau was reached, the buffer was changed to PBS to monitor the dissociation of the protein. Protein addition was performed in an open loop to ensure a constant ENTH concentration in the bulk solution. Changes of the optical thickness were 
converted into physical thickness changes according to equation 3.46 using a refractive index of $n_{\text {lipid }}=n_{\text {protein }}=1.47$ for the lipid bilayer and the protein layer. 106, 107]

\subsection{Scanning probe microscopy}

Scanning probe microscopy is a class of techniques in which interactions between a probe and a sample are measured point-wise to extract various information such as mechanical or material properties and to reconstruct an image from the measured data. The scanning tunneling microscope invented in 1981 was the first scanning probe microscope and was followed by the development of different other techniques such as atomic force microscopy. ${ }^{[108]}$ Microscopes belonging to this class achieve up to atomic resolution and use various physical processes and effects such as the tunnel effect, electrostatics and repulsive and attractive interactions between sample and probe.

\subsubsection{Atomic force microscopy}

Invented by Binning, Gerber and Quate in 1985, the atomic force microscope allows the characterization of various specimen including in vivo samples. 109 As it provides a high resolution and allows to measure small forces relevant for biological applications it is widely used for the analysis of biophysical processes such as ligand-receptor interactions and protein unfolding. 110, 111]

Atomic force microscopes measure the deflection of a cantilever interacting with a sample. Light of a diode is focused on the cantilever and reflected onto a four quadrant photo diode. Forces between sample and tip deflect the cantilever and changes in the sub-nanometer range are detectable. When approaching the sample, attractive van-der-Waals interactions between cantilever and sample occur with decreasing distance. When further decreasing the distance, repulsive interactions rapidly increase the force affecting the cantilever. As a first approximation, the interactions between cantilever and sample can be described by a Lennard-Jones potential (equation 3.49). 


$$
V=4 \epsilon\left(\left(\frac{\sigma_{\mathrm{L}-\mathrm{J}}}{h}\right)^{12}-\left(\frac{\sigma_{\mathrm{L}-\mathrm{J}}}{h}\right)^{6}\right)
$$

The potential $V$ is a function of the distance of the cantilever to the sample $h$, the magnitude of the potential well $\epsilon$ and the distance $\sigma_{\mathrm{L}-\mathrm{J}}$ at which repulsive and attractive interactions compensate each other. The different operation modes of in atomic force microscopy (AFM) require different interaction potentials and therewith different distances between cantilever and sample (Figure 3.13). The contact mode $(\mathrm{C})$ requires a permanent contact of the tip to the sample applying a constant force using a feedback loop. The intermediate-contact mode (IC) is characterized by only short contacts between cantilever and sample and allows sensitive probing. The non-contact mode (NC) can provide a very high resolution but is only suitable for very flat samples as the cantilever deflection is measured at a constant distance.

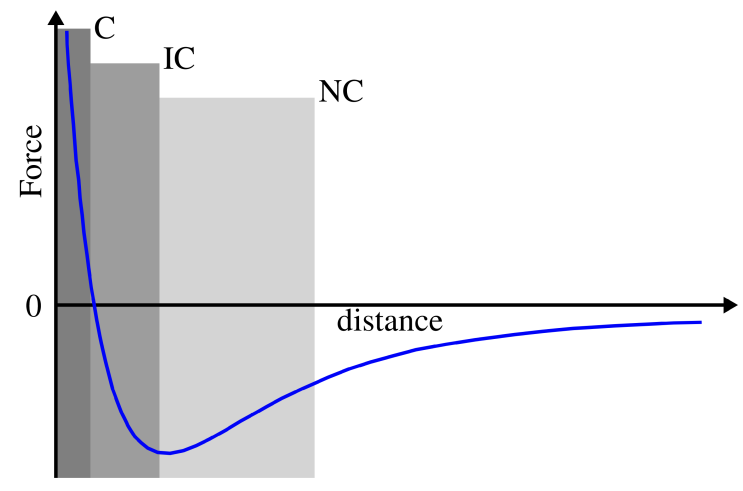

Figure 3.13: Schematic illustration of the interaction potential between cantilever and sample and the regime of the different operation modes of an atomic force microscope. C: contact mode, IC: intermediate-contact mode, NC: non-contact mode.

In force spectroscopy the deflection of the cantilever is measured as a function of the cantilever movement. Force-distance measurements allow to investigate processes at a single molecule level such as protein unfolding and rupture of covalent bonds. 112] Atomic force microscopy was also used for local probing and ensemble measurements to analyze the mechanics of cellular and artificial membranes. [35, 113-115] Information about the mechanical properties of the sample can be extracted from force-distance curves. A typical force-distance curve is shown in Figure 3.14. The cantilever approaches the substrate (Figure 3.14 A) 
and at a certain distance attractive electrostatic or van-der-Waals interactions can lead to a snap on of the cantilever which is then in contact with the specimen (Figure 3.14 B). By moving the cantilever further towards the sample, a force is applied and the response of the system is measured (Figure $3.14 \mathrm{C}$ ). After reaching a predefined force, the cantilever is retracted. Adhesion can result in a bending of the cantilever maintaining contact to the surface. When being further retracted the retraction force exceeds the adhesion force and the cantilever looses contact to the surface (snap off, Figure 3.14D). The cantilever is then further retracted until the predefined tip-sample separation is reached (Figure 3.14 E). This procedure can be repeated to acquire a force-volume image containing mechanical information of each probed location.

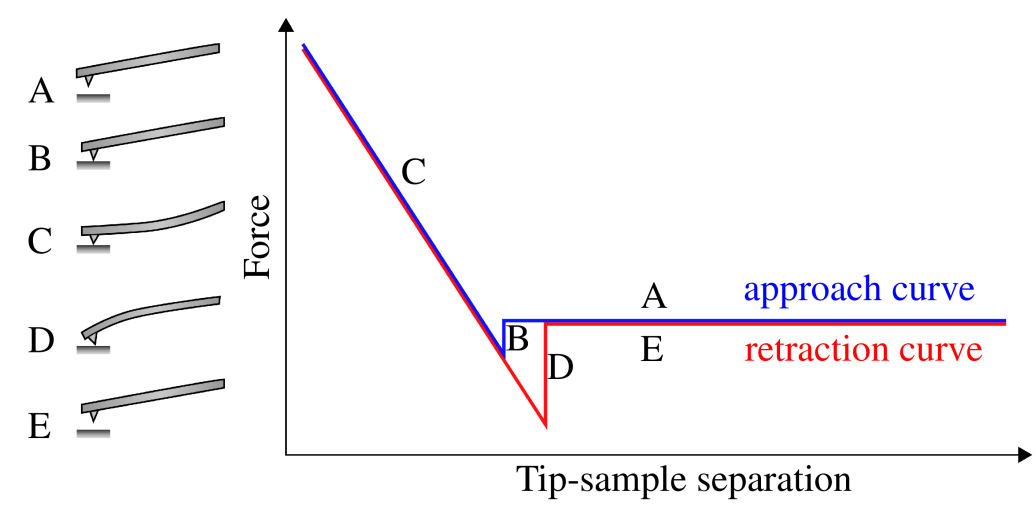

Figure 3.14: Schematic illustration of the measurement of a force-distance curve.

As the atomic force microscope measures the deflection $z_{\mathrm{c}}$ of a cantilever, a conversion is required to obtain the applied force. According to Hooke's law the force $F$ of an ideal, linear elastic spring is given by:

$$
F=-k_{\mathrm{c}} \cdot z_{\mathrm{c}}
$$

with $k_{\mathrm{c}}$ being the spring constant and $z_{\mathrm{c}}$ the deflection of the spring. The deflection is then converted into the tip-sample separation $h$ by subtracting the cantilever deflection $z_{\mathrm{c}}$ and sample indentation $z_{\mathrm{s}}$ from the piezo movement $z_{\mathrm{p}}$ :

$$
h=z_{\mathrm{p}}-z_{\mathrm{c}}-z_{\mathrm{s}}
$$


For incompressible materials is $z_{\mathrm{s}} \ll z_{\mathrm{c}}$ and can be neglected. The spring constant of the cantilever was determined by the method of Hutter and Bechhofer measuring the intensity of the thermal noise of the cantilever. [116] Assuming a harmonian oscillator, introducing two correction parameters accounting for deviations from the ideal behavior allows the calculation of the thermal noise as a function of the mean square displacement of the cantilever $\left\langle z_{c}^{2}\right\rangle$ to:

$$
k_{\mathrm{c}}=\frac{\beta k_{\mathrm{B}} T}{\chi^{2}\left\langle z_{\mathrm{c}}^{2}\right\rangle} \text {. }
$$

Hereby $\chi$ corrects for the cantilever not oscillating entirely free but being endloaded. [117] As only a single mode of a real cantilever is measured for the determination of the thermal noise, the parameter $\beta$ was introduced as a second correction factor. [118]

When investigating the mechanical properties of pore-spanning membranes, contributions from membrane bending, stretching and the lateral membrane tension have to be considered. However, for small indentations of circular porespanning membranes it was shown that the force $F$ increases linearly as a function of the indentation depth $h$ and that membrane bending and stretching can be neglected. [113, 119] The lateral membrane tension $\sigma$ is governed by the adhesion of the lipids to the pore rim and can then be extracted from:

$$
h=\frac{F}{4 \pi \sigma}\left[1-\ln \left(\frac{F r_{\text {tip }}^{2}}{2 \pi \sigma r_{\text {pore }}^{2}}\right)\right]
$$

with $r_{\text {tip }}$ and $r_{\text {pore }}$ being the radius of the cantilever tip and the pore radius. [120]

\section{Experimental procedure}

Pore-spanning membranes on porous glass substrates were generated as described in Section 3.5.2. Fluorescence microscopy was used for localization of the membrane-covered pores. Force volume images of $16 \times 16$ to $64 \times 64$ forcedistance curves were measured by a Nanowizard 2 (JPK Instruments AG, Berlin, Germany) using a cantilever with a spring constant of $k_{\mathrm{c}}=0.01 \mathrm{~N} / \mathrm{m}$ (MLCT, Bruker AFM Probes, Calle Tecate, USA). Areas ranging from $8 \times 8$ to $30 \times 30 \mu \mathrm{m}$ were probed with velocities ranging from 2 to $8 \mu \mathrm{m} / \mathrm{s}$ each probing a distance of 1 to 
$5 \mu \mathrm{m}$. Indentation was stopped after applying a force of 200 to $900 \mathrm{nN} / \mathrm{m}$. The linear regime of the force-distance curves was fitted for small indentation depths $(<300 \mathrm{~nm})$ and the lateral membrane tension was then extracted by numerically solving equation 3.53 for the measured dependence of force to the indentation depth.

\subsubsection{Scanning ion conductance microscopy}

Scanning ion conductance microscopy (SICM) was invented by Hansma et al. in 1989 and allows to probe samples in a contact-free manner. 121, 122 To image the topology of a surface, the electrical current is recorded by a pipette with a small aperture and an internal electrode. A second electrode is in contact with a conducting bath solution. The inner diameter of the glass pipettes used is typically 30 to $100 \mathrm{~nm}$ leading the lateral resolutions in the same range. 123 The ion current $I$ at a constant voltage $U$ between pipette and counter electrode is determined by the total resistance $R$ which is the sum of the pipette resistance $R_{\mathrm{p}}$ and the access resistance $R_{\text {acc }}$ between pipette and sample. The resistance of the pipette is a function of its geometry (Figure 3.15) and can be approximated to:

$$
R_{\mathrm{p}}=\frac{\rho l_{\mathrm{p}}}{\pi r_{\mathrm{p}} r_{\mathrm{i}}},
$$

with $\rho$ being the specific electrical resistivity of the electrolyte, $r_{\mathrm{p}}$ the inner radius of the pipette, $r_{\mathrm{i}}$ the inner radius of the pipette opening and $l_{\mathrm{p}}$ the length of the pipette. The overall resistance equals the tip resistance at large tip-sample separations (equation 3.55).

$$
R_{\infty} \approx R_{\mathrm{p}} \text { for } h \rightarrow \infty
$$

The access resistance is a function of the pipette's inner tip radius $r_{i}$, the outer radius of the tip base $r_{\mathrm{o}}$ and the specific electrical resistivity of the electrolyte. It is strongly dependent on the distance between pipette and sample surface $h$ (equation 3.56.

$$
R_{\mathrm{acc}} \approx \frac{3 \rho \ln \left(\frac{r_{\mathrm{o}}}{r_{\mathrm{i}}}\right)}{2 \pi h}
$$


The measured current $I$ as a function of $h$ is then given by:

$$
I(h)=\frac{U}{R}=\frac{U}{R_{\mathrm{p}}+R_{\mathrm{acc}}}=I_{\max }\left(1+\frac{3 \ln \left(\frac{r_{\mathrm{o}}}{r_{\mathrm{i}}}\right) r_{\mathrm{p}} r_{\mathrm{i}}}{2 l_{\mathrm{p}} h}\right)^{-1}
$$

Equation 3.57 approaches a constant current for large tip-sample distances which is given by: $I_{\max }=U / R_{\mathrm{p}}$. The current as a function of the tip-sample distance is plotted for a typical pipette geometry and applied voltage in Figure 3.15. In contrast to atomic force microscopy, SICM allows to probe topologies with complex features in a contact-free manner as the pipette is retracted when the current drops below a defined threshold. [122]

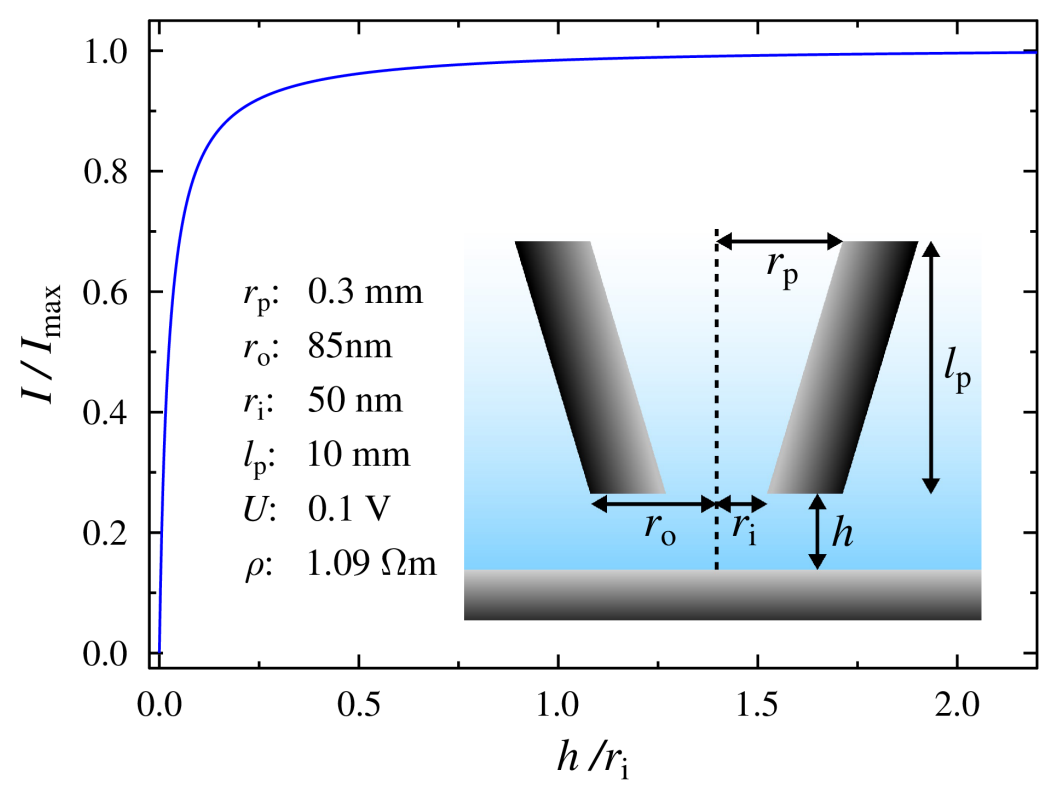

Figure 3.15: Current as a function of the tip-sample distance for a typical pipette geometry. Derived from Bhushan et al. 123

\section{Experimental procedure}

Pipettes were produced from glass capillaries (borosilicate glass, outer diameter: $1 \mathrm{~mm}$, inner diameter: $0.58 \mathrm{~mm}$, length: $8 \mathrm{~cm}$, Science Products, Hofheim, Germany) using a P-1000 micropipette puller (Sutter Instruments, Novato, USA, settings: heat: ramp 10, ramp 523, pull: 55, velocity: 110, delay: 80 , pressure: 400, 
loops: 1, time 10.4 to 10.5 s). Pipettes were filled with degassed PBS. Electrodes $(\mathrm{Ag} / \mathrm{AgCl})$ were prepared using an aqueous solution of $\mathrm{NaClO}(1.6 \mathrm{M})$ for oxidation of a silver wire and were placed in the pipette and bulk solution.

The hopping mode was used for imaging which is similar to the measurement of force volume images by AFM. The pipette was immersed into the buffer and then moved towards the sample. After approaching it, the pipette is further moved towards the sample until the ion current drops by a defined threshold which was typically set to 0.3 to $0.4 \%$ of $I_{\max }$. The pipette was then retracted, the sample was moved by a piezo element and the approach cycle was repeated (Figure 3.16).

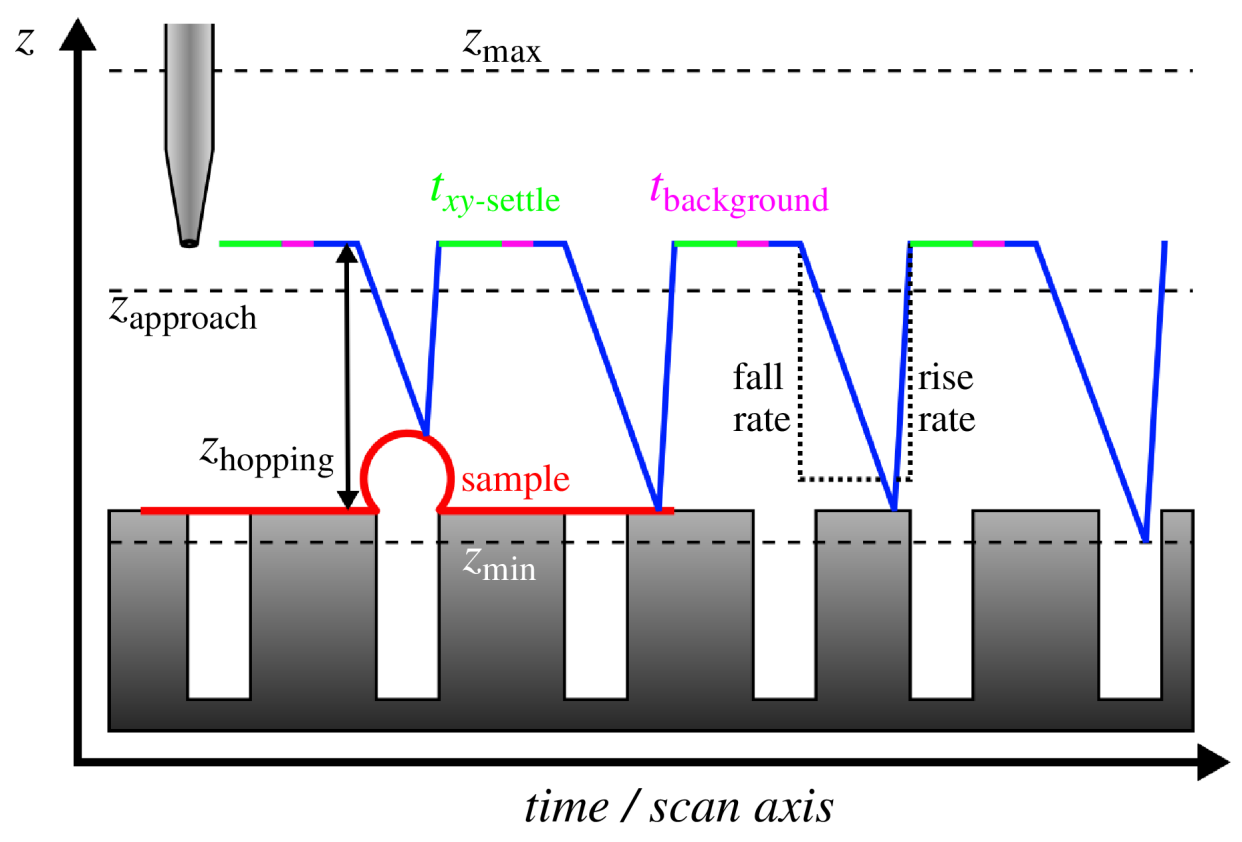

Figure 3.16: Schematic illustration of the operation principle of SICM (hopping mode).

Probing of a non-covered pore could result in breakage of the pipette as it is moved deeply into the pore and is then diagonally retracted. To avoid breaking of the pipette, a minimum $z$-height $z_{\text {min }}$ was set. The maximum $z$-height marked the upper limit for scanning and the hopping height defined the retraction distance of the pipette between two data points. A hopping height of $1 \mu \mathrm{m}$ was used to enable imaging of protruded pore-spanning membranes on porous substrates with pore diameters of $850 \mathrm{~nm}$. Areas ranging from $20 \times 20 \mu \mathrm{m}$ to $40 \times 40 \mu \mathrm{m}$ were imaged with a resolution of $128 \times 128$ pixels to $512 \times 512$ pixels, respectively. Typical parameters used for imaging are summarized in Table 3.3 . 
Table 3.3: Experimental parameters used for the SICM experiments.

\begin{tabular}{rrl}
\hline Parameter & value & unit \\
\hline U & 0.2 & $\mathrm{~V}$ \\
immerse threshold & 0.2 & $\mathrm{~V}$ \\
imaging threshold & $0.3-0.4$ & $\%$ \\
image size & $20 \times 20-40 \times 40$ & $\mu \mathrm{m}$ \\
image resolution & $128 \times 128-512 \times 512$ & pixel \\
scan direction & unidirectional & \\
hopping height & 1 & $\mu \mathrm{m}$ \\
approach height & 12.5 & $\mu \mathrm{m}$ \\
$z_{\mathrm{min}}$ & 12 & $\mu \mathrm{m}$ \\
$z_{\mathrm{max}}$ & 25 & $\mu \mathrm{m}$ \\
time background measurement & 15 & $\mathrm{~ms}$ \\
fall rate & 5 & $\mathrm{~ms}$ \\
rise rate & $15-25$ & $\mathrm{~nm} / \mathrm{s}$ \\
approach speed & $250-400$ & $\mathrm{~nm} / \mathrm{s}$ \\
immerse speed & $1-10$ & $\mu \mathrm{m} / \mathrm{s}$ \\
& 200 & $\mu \mathrm{m} / \mathrm{s}$ \\
\hline
\end{tabular}

\subsubsection{Scanning electron microscopy}

Scanning electron microscopy (SEM) uses an electron beam for probing of electrically conducting samples. As electrons have a smaller wavelength compared to visible light, the achievable resolution is greatly improved. SEM is able to image with a very high field depth resulting in very detailed images of samples with varying topology. However, a main drawback is the requirement of conducting samples and a high vacuum which prohibits imaging of living samples or in an aqueous environment.

A scanning electron microscope is schematically shown in Figure 3.17 A. Electrons are generated from the electron gun for which usually a tungsten filament, a $\mathrm{LaB}_{6}$ rod cathode or a field emission gun is used. ${ }^{[124]}$ The electron beam is accelerated by a voltage typically ranging from 0.2 to $40 \mathrm{kV}$, is then focused onto the sample by magnetic lenses and produces different types of electrons depending on the energy of the electron beam. As a result of inelastic collisions, secondary electrons (SEs) are produced. They originate in proximity to the substrate surface and are detected by an Everhart-Thornley detector. [125] Backscattered electrons (BSEs) result from elastic collisions of the electrons from the electron beam with the electrons at 
the sample surface. Elements with a high mass backscatter the electrons of the beam more efficiently and appear bright in images when BSE are detected. Core scattering and inner shell ionization produce X-rays whose detection enables to probe the chemical composition of the sample. When the electron beam excites secondary electrons of e.g. a semiconductor they can lead to emission of light in the visible spectrum (cathode luminescence). The different types of electrons and detectable radiation by the SEM are schematically shown in Figure 3.17B.
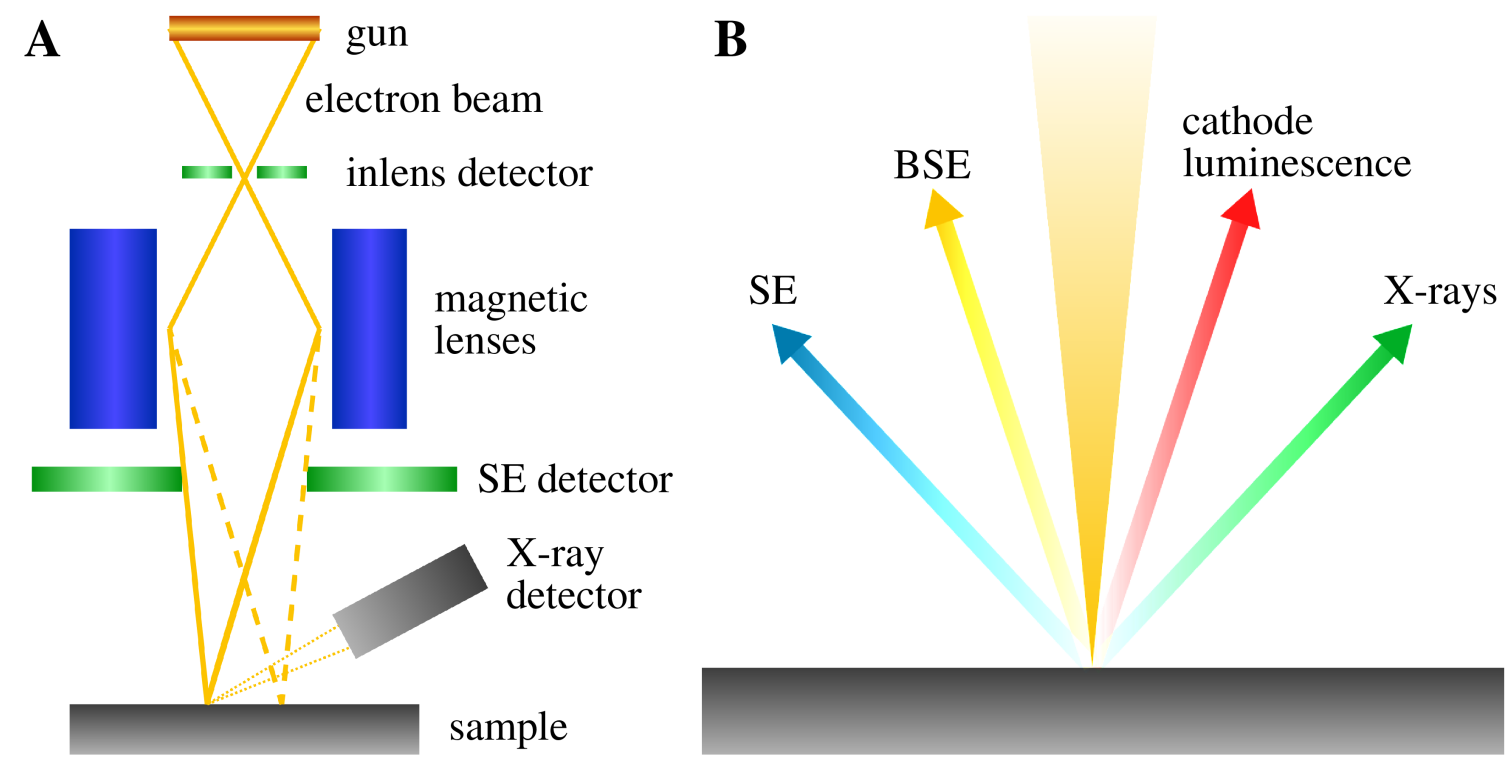

Figure 3.17: A: Schematic illustration of the operation principle of scanning electron microscopy. B: Types of signals produced in scanning electron microscopy.

\section{Experimental procedure}

Porous substrates were imaged after removal of the protrective varnish using a LEO supra-35 microscope (Carl Zeiss, Jena, Germany). An electron beam with an acceleration voltage of $5 \mathrm{kV}$ was used to image the substrates at pressures smaller than $5 \cdot 10^{-10}$ mbar. Secondary electrons were detected by an SE-detector perpendicular to the sample surface using a $30 \mu \mathrm{m}$ aperture. 


\subsection{Data evaluation}

\subsubsection{Determination of the GUV geometry}

SDCLM was used to image $z$-stacks of GUVs adhering to an avidin coated glass surface. GUVs were imaged with $10 \%$ laser power, a distance of 150 to $250 \mathrm{~nm}$ between the slices, 20 ms exposure time and a LP496 ET long-pass filter to detect the full emission spectrum of the fluorophore used. With these parameters the point spread function $\left(d_{F W H M, z} \approx 800 \mathrm{~nm}\right)$ was oversampled in $z$-direction increasing the data available for further evaluation while maintaining a short imaging time per $z$-stack ( 0.5 to $1.5 \mathrm{~min}$ ) allowing to acquire higher statistics.

To measure the GUV geometry a custom written Matlab script was used to analyze hundreds of imaged GUV-z-stacks semi-automatically. The evaluation procedure is schematically shown in Figure 3.18. Images were loaded into Matlab and the metainformation was extracted from the corresponding txt-file. The intensity $I_{\text {raw }}$ of the $z$-stack was corrected for inhomogeneous illumination resulting from the intensity profile of the widened excitation laser. The intensity profile of the spinning disc is dependent on the wavelength of illumination allowing the usage of dyes with similar spectral properties for calibration compared to the dye used for imaging. Imaging a solution of sulforhodamine 101 which yielded a bright image $\left(I_{\text {bright }}\right)$ and the dark image $\left(I_{\mathrm{dark}}\right)$ was obtained by reading out the camera without illumination. To avoid any dependence of the corrected image $\left(I_{\text {corr }}\right)$ on the absolute intensity of the bright image, the camera-offset-corrected bright image was normalized to its maximum intensity. The corrected image was calculated using equation 3.58 .

$$
I_{\text {corr }}=\frac{I_{\text {raw }}-I_{\text {dark }}}{\frac{I_{\text {bright }}-I_{\text {dark }}}{I_{\text {bright,max }}-I_{\text {dark }}}} .
$$

The equatorial slice of the GUV was then selected by user input. Next, the possibility was given to define regions each containing only a single GUV which was essential as the further evaluation script was written for a single GUV in each region. The Matlab workspace, containing the corrected image was saved and all user input was saved in a separate global info file. The evaluation procedure described so far was repeated until the parameters were defined for all $z$-stacks within a folder. 


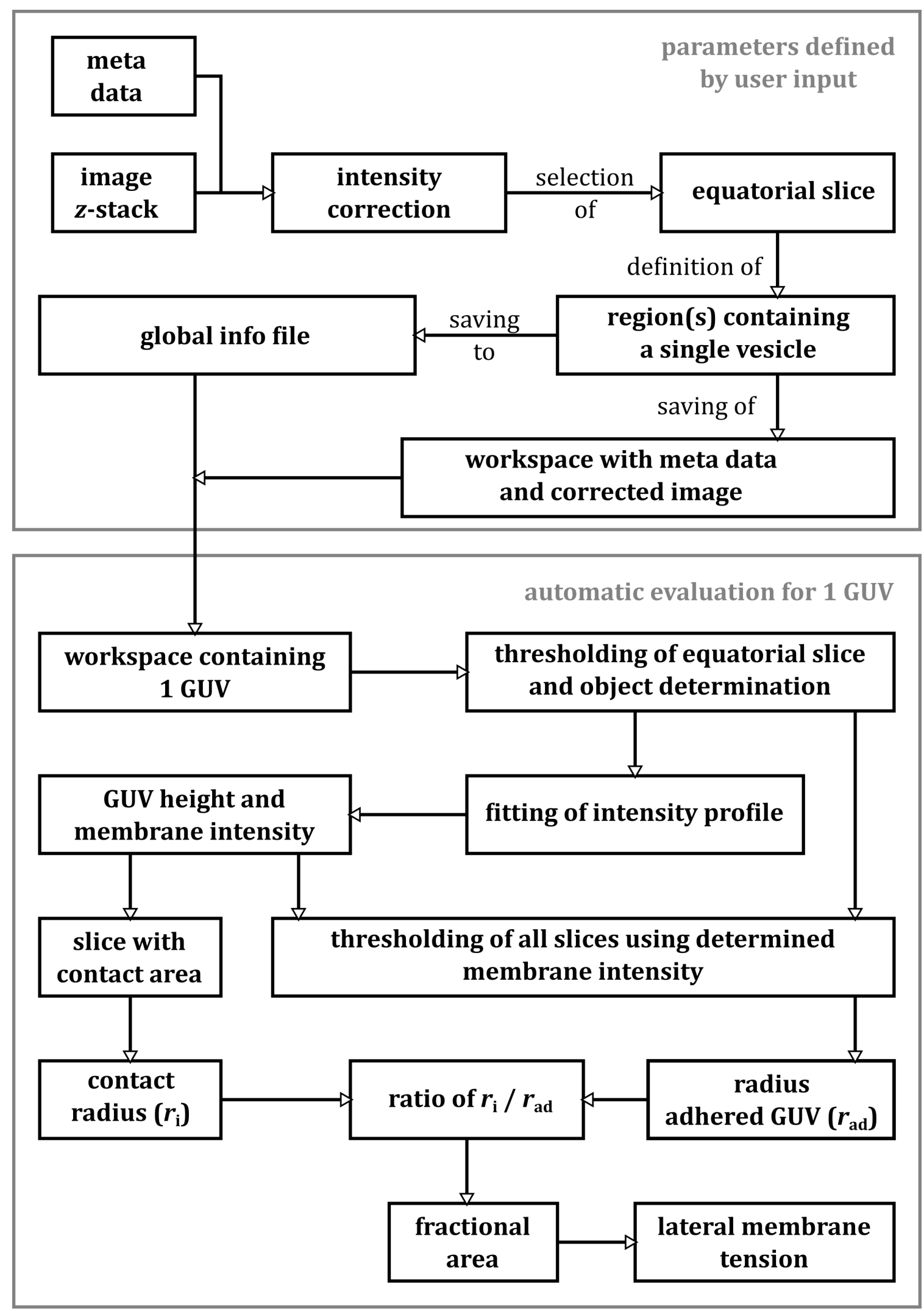

Figure 3.18: Schematic illustration of the script used for data evaluation to determine the GUV geometry. 
Consecutively, for each $z$-stack the corresponding parameter set and workspace were automatically loaded and evaluated. The image of the equatorial slice of each $z$-stack, previously defined by user input, was thresholded and the largest object was detected using a watershed algorithm. The center of mass of this object was used to create a region of interest (ROI) and the intensity profile within this region was measured in $z$-direction. The intensity profile showed two peaks resulting from the upper and lower GUV membrane. Each peak was fitted with a Gaussian function to obtain the position and intensity of the upper and lower GUV membrane. The position of the lower GUV membrane was used to determine the slice of the $z$-stack containing the contact area. To avoid pinhole crosstalk resulting from the membrane fluorescence above the contact area, a slice below the determined membrane position was used for thresholding. The distance of this slice was set to 300 to $750 \mathrm{~nm}$ by user input. Otherwise, the pinhole crosstalk would result in an overestimation of the size of the contact area. The contact area was thresholded and the contact radius $\left(r_{\mathrm{i}}\right)$ was calculated, assuming a circle.

The intensity of the upper membrane was used to threshold each slice of the $z$-stack. Logical summation of the individual thresholded slices was used to create a black and white image of the maximum GUV extent. The largest object detected by a watershed algorithm corresponds to the GUV equatorial plane and the radius of the adhered GUV $\left(r_{\mathrm{ad}}\right)$ was calculated from the circular area. By thresholding each slice and not only the previously defined equatorial slice, the robustness of the determination was improved. The radius of the contact area was then normalized to the radius of the adhered GUV. The increase in area was calculated from the determined ratio of $r_{\mathrm{i}} / r_{\mathrm{ad}}$ and the resulting lateral tension was then calculated as described in Section 4.5.1.

\subsubsection{Influence of electrostatics and osmotic gradients on the GUV geometry}

The excess area of GUVs is mainly dependent on the vesicle size and the presence of an osmolarity gradient which can suppress or enhance undulations. The theoretical model used for calculation of the lateral membrane tension (Section 4.5.1) is valid for the absence of osmolarity gradients. Therefore, iso-osmolarity between GUV interior and exterior was indispensable. GUVs were formed by electro-formation since this method yields more unilamellar GUVs than swelling of dried lipid films 
in buffer. [126] However, the electro-formation protocol requires the absence of high amounts of charged lipids or ions in solution during the formation process. [127] GUVs were formed in sucrose solution (sucrose, $298 \mathrm{mOsmol} / \mathrm{kg}$ ) and added to sucrose buffer (sucrose, $3 \mathrm{~mm} \mathrm{MgCl}_{2}, 2 \mathrm{~mm}$ HEPES, pH 7.4, $298 \mathrm{mOsmol} / \mathrm{kg}$ ). Sucrose solution and sucrose buffer had an almost identical composition and were adjusted to the same osmolarity ensuring iso-osmolarity between GUV interior and exterior which was required to calculate the vesicles membrane tension using the theoretical framework described in Section 4.5.1. Evaporation of water would cause a rise of osmolarity of the bulk solution leading to an increase of the vesicles' excess area. This would translate to larger contact areas upon adhesion and therefore in an overestimation of the vesicles' membrane tension. To avoid evaporation of water a humidity chamber was mounted to the objective used (Figure 3.19 A). As an indirect indicator for the vesicles' excess area, the contact area of adhering vesicles was imaged as a function of time. Without humidity chamber a large increase of the contact area was measured after 90 min (Figure 3.19 C) while it remained almost constant when the humidity chamber was used (Figure 3.19 B).
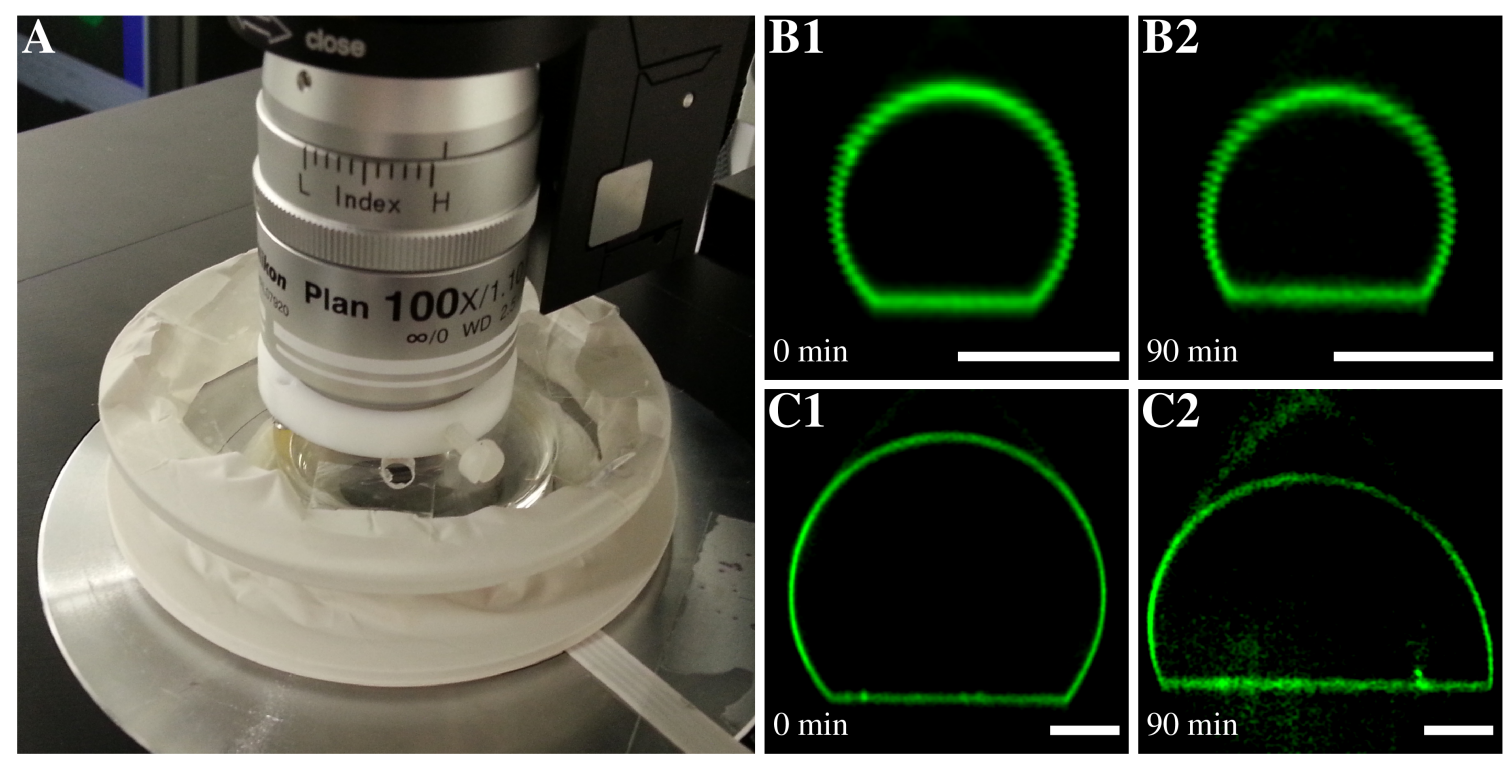

Figure 3.19: A: Humidity chamber with a small hole for protein addition mounted onto the objective. B+C: Z-profiles of GUVs (DOPC/DOPE/cap-biotin-DOPE/Atto488-DPPE, 67:30:2:1) adhering to an avidin coated surface in the presence of $3 \mathrm{~mm} \mathrm{Mg}{ }^{2+}$ imaged by SDCLM. The vesicle geometry remains unchanged when a humidity chamber was used (B) whereas the GUV flattens without humidity chamber due to the increasing osmolarity of the bulk solution caused by evaporation of water $(\mathbf{C})$. Scale bars: $5 \mu \mathrm{m}$. 
Additionally, electrostatic interactions were identified to influence the adhesion strength. To check how sensitive biotinylated GUVs adhering to an avidin coated surface change their contact area as a result of the presence of ions, a small volume $(100 \mu \mathrm{L})$ of iso-osmolar $\mathrm{NaCl}$ buffer was added to the GUV solution $(V=3 \mathrm{~mL})$. Even though iso-osmolar conditions were maintained and the final $\mathrm{NaCl}$ concentration was only $4 \mathrm{~mm}$, the contact area of the vesicles increased about $25 \%$ (Figure 3.20). To avoid changes in contact area due to addition of ions the protein buffer (PBS) was changed to sucrose buffer with identical composition as used for the experiment. By using a humidity chamber and buffers with almost identical composition, it was possible to adjust the experimental conditions to create and maintain iso-osmolar conditions for the duration of the experiments. This was also essential for the applicability of the theoretical framework used for the determination of the membrane tension (Section 4.5.1).
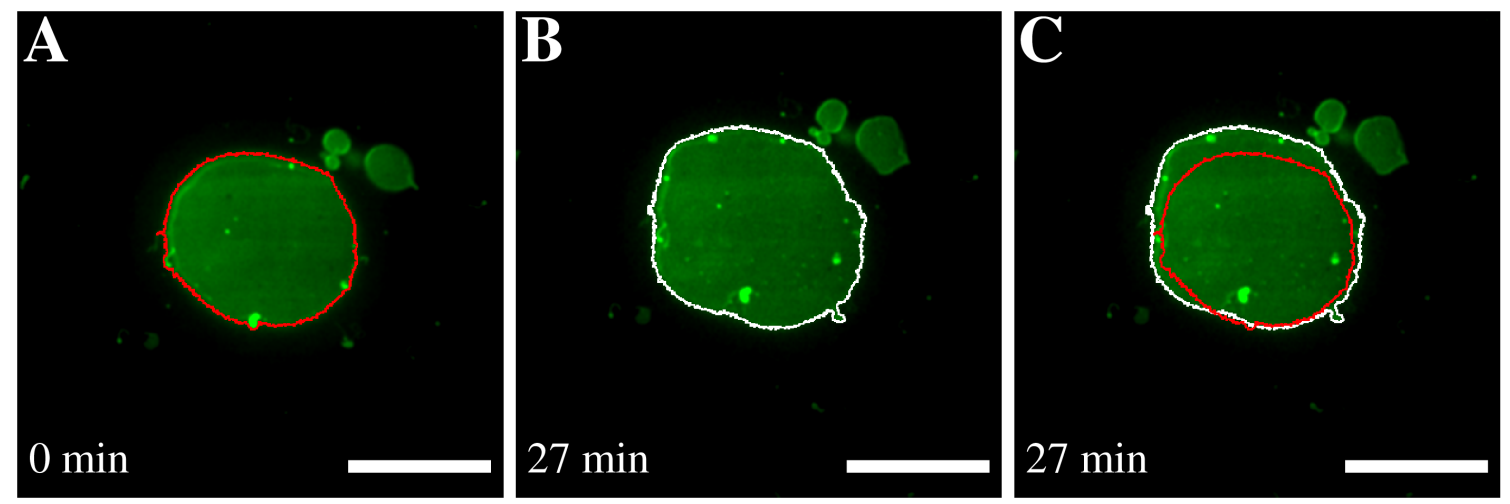

Figure 3.20: A: SDCLM image of the contact area of a GUV (DOPC/DOPE/cap-biotinDOPE/Atto488-DPPE/PIP $2,66.2: 30: 2: 1: 0.8$ ) adhering to an avidin coated glass surface in sucrose buffer with $3 \mathrm{~mm} \mathrm{Mg}^{2+}$. The boundary of the contact area is shown in red. B: Addition of $\mathrm{NaCl}\left(100 \mu \mathrm{L}\right.$ of iso-osmolar $\mathrm{NaCl}$ to $\left.3 \mathrm{~mL}, c_{\text {final }}=4 \mathrm{~mm}\right)$ leads to a significant increase of the contact area. The boundary of the contact area is highlighted in white. C: Overlay of the boundary of the contact area before $(\mathbf{A}$, red) $\mathrm{NaCl}$ addition and image $\mathbf{B}$. 


\section{Results}

\subsection{Determination of the dissociation constant of ENTH binding to $\mathrm{PIP}_{2}$}

It is a topic of current discussion if protein crowding is a major contribution to the curvature generating properties of proteins involved in endocytosis. [8, 128, 129] The dissociation constant $K_{\mathrm{D}}$ of ENTH binding to its receptor lipid $\mathrm{PIP}_{2}$ was measured to determine the protein concentration which will result in saturation of the receptor lipids with protein. Reported dissociation constants of ENTH range from $2.6 \mathrm{~nm}$ to $850 \mathrm{~nm}$ dependent on the chosen experimental conditions. [13, 47, 50, 51] Therefore, two different and independent techniques were used to measure the dissociation constant for the experimental conditions and membrane topologies used in this thesis. Reflectometric interference spectroscopy (RIfS) was used to measure binding of ENTH to planar solid-supported membranes. Surface plasmon resonance (SPR) spectroscopy experiments with immobilized vesicles of different lipid compositions were performed to determine the dissociation constant of ENTH binding to curved membranes.

\subsubsection{Reflectometric interference spectroscopy}

Reflectometric interference spectroscopy (RIfS) experiments were conducted to analyze binding of ENTH to planar solid-supported membranes. Small unilamellar vesicles (SUVs) composed of POPC/PIP $2(90: 10,0.8 \mathrm{mg} / \mathrm{mL})$ in citrate buffer (20 mm Na-citrate, $50 \mathrm{~mm} \mathrm{KCl,0.1} \mathrm{mм} \mathrm{EDTA,} 0.1 \mathrm{~mm} \mathrm{NaN}_{3}, \mathrm{pH} 4.8$ ) were added to a silicon wafer with a hydrophilic silicon dioxide layer of $5 \mu \mathrm{m}$. The optical thickness $(\triangle O T)$ increased after vesicle addition indicating spreading of vesicles and the formation of a lipid bilayer (Figure 4.1A). Bilayer formation was completed 
after about 10-15 min. An average change in optical of $6.5 \pm 0.3 \mathrm{~nm}(n=7)$ was measured and converted to a physical thickness of $4.4 \pm 0.2 \mathrm{~nm}$ (equation 3.46 ). The citrate buffer with a $\mathrm{pH}$ of 4.8 was used to reduce the negative charges of $\mathrm{PIP}_{2}$ and the $\mathrm{SiO}_{2}$ surface and was required for the formation of the lipid bilayer. 105] After formation of a stable baseline bovine serum albumin (BSA, $c_{\mathrm{BSA}}=1 \mathrm{mg} / \mathrm{mL}$ ) was added to block hydrophobic defects to which ENTH could bind unspecifically (b, Figure 4.1A). No change in the optical thickness was measured after addition of BSA indicating a defect-free membrane as unspecific binding of BSA to membrane defects would have increased the optical thickness. After addition of ENTH (c, Figure $4.1 \mathrm{~A}, c_{\mathrm{ENTH}}=3 \mu \mathrm{M}$ ) the optical thickness increased as a result of protein binding to the membrane. Rinsing with PBS leads to the dissociation of ENTH molecules and the optical thickness dropped to an equilibrium value providing the fraction of non-reversibly bound protein (a, Figure 4.1 A). The optical thickness dropped to almost the value before ENTH addition and the fraction of reversibly bound ENTH was determined to $93 \%$.
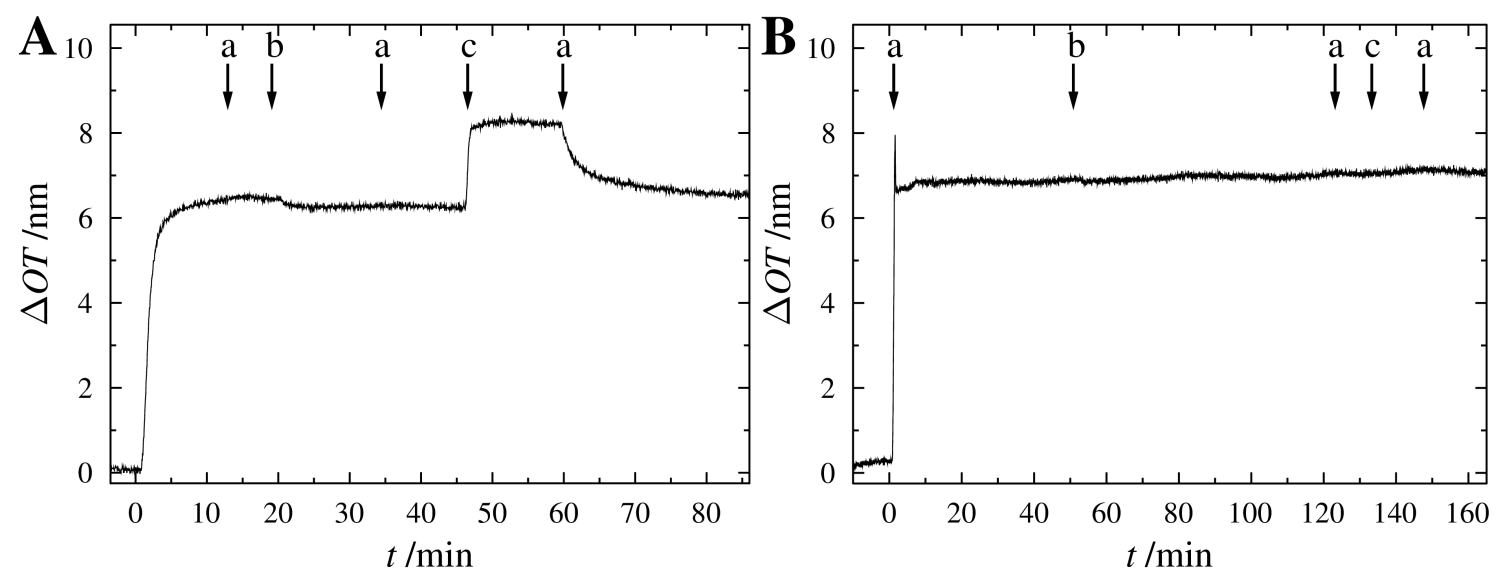

Figure 4.1: Representative time resolved change in optical thickness observed by means of RIfS. a: rinsing with PBS b: addition of BSA $\left(c_{\mathrm{BSA}}=1 \mathrm{mg} / \mathrm{mL}\right)$. c: addition of ENTH $\left(c_{\text {ENTH }}=3 \mu \mathrm{M}\right)$. SUVs $\left(A:\right.$ POPC $/ P_{2}, 90: 10, c=1 \mathrm{mg} / \mathrm{mL}, \mathbf{B}:$ POPC, $\left.c=1 \mathrm{mg} / \mathrm{mL}\right)$ were added at $t=0 \mathrm{~min}$.

To exclude unspecific binding of ENTH to the lipid bilayer the experiment was repeated with SUVs (POPC, $0.8 \mathrm{mg} / \mathrm{mL}$ ) lacking the receptor lipid $\mathrm{PIP}_{2}$. After addition of SUVs the optical thickness increased to $6.4 \pm 0.3 \mathrm{~nm}(n=3)$ which was converted to a physical thickness of $4.4 \pm 0.2 \mathrm{~nm}$ (equation 3.46). Bilayer formation was completed after approximately $5 \mathrm{~min}$. This was faster compared to spreading 
of the SUVs containing the receptor lipid. As BSA addition did not change the optical thickness, it was concluded that no BSA adsorbed to membrane defects and the formed lipid bilayer was defect-free. In contrast to the experiments containing the receptor lipid $\mathrm{PIP}_{2}$, addition of ENTH $\left(c_{\mathrm{ENTH}}=3 \mu \mathrm{M}\right)$ did not change the optical thickness. Hence, no binding of ENTH to a pure POPC bilayer occurred highlighting the specificity of the ENTH-PIP 2 interaction (c, Figure 4.1 B). Rinsing with PBS (a, Figure 4.1 B) did not affect the change in optical thickness and the constant $\triangle O T$ also shows the long term stability of the membrane.

To determine the dissociation constant of ENTH binding to $\mathrm{PIP}_{2}$ the Langmuir adsorption model was used. It assumes an energetically homogeneous surface, equality of all binding sites, the formation of a monolayer and the absence of phase transitions. The large reversibly bound fraction of ENTH is a prerequisite for the evaluation using the Langmuir adsorption model. Changes in optical thickness were plotted versus the ENTH concentration (Figure 4.2).

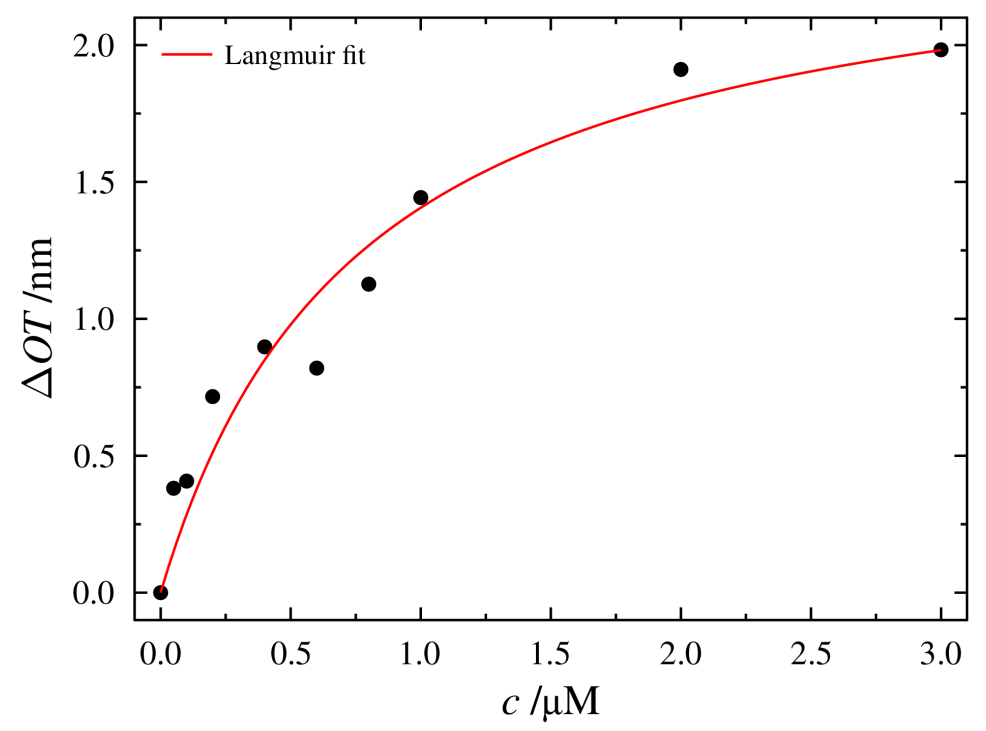

Figure 4.2: Isotherm of ENTH binding to a planar solid-supported lipid bilayer composed of POPC/PIP 2 (90:10) in PBS measured by means of RIfS. The binding isotherm includes 9 independent measurements with one protein addition per measurement. Data were fitted according to the Langmuir adsorption model using equation 4.1 .

The measured optical thicknesses as a function of the ENTH concentration are well described by the Langmuir adsorption model (equation 4.1 ) as indicated by the correlation coefficient of $R^{2}=0.93$. 


$$
\frac{\theta}{\theta_{\max }}=\frac{c_{\mathrm{ENTH}}}{K_{\mathrm{D}}+c_{\mathrm{ENTH}}}
$$

The dissociation constant of ENTH binding to planar membranes composed of POPC/PIP 2 (90:10) was determined by fitting the binding isotherm according to the Langmuir adsorption model.[130] A dissociation constant of $0.8 \pm 0.2 \mu \mathrm{M}$ was determined with an error resulting from the fit uncertainty.

The change in optical thickness measured by RIfS can be converted into a physical thickness change and is proportional to the surface coverage with protein $(\theta)$. A maximum optical thickness change of $\Delta O T_{\max }=2.5 \pm 0.3 \mathrm{~nm}$, resulting from binding of ENTH to a planar membrane composed of POPC/PIP 2 (90:10), was determined by fitting equation 4.1 to the binding isotherm. It was converted into a physical thickness of $d_{\max }=1.7 \pm 0.2 \mathrm{~nm}$ using equation 3.46 and a refractive index of 1.47 for a protein layer. ${ }^{[107]}$ The determined physical thickness of ENTH $\left(d_{\max }=1.7 \pm 0.2 \mathrm{~nm}\right)$ at maximum surface coverage matches the height of the protein's crystal structure assuming a protein density on the membrane of $56 \%$ according to the scaled particle theory. [48, 131, 132]

\subsubsection{Surface plasmon resonance spectroscopy}

To obtain a dissociation constant of ENTH binding to $\mathrm{PIP}_{2}$ in curved membranes surface plasmon resonance (SPR) spectroscopy experiments with large unilamellar vesicles (LUVs) immobilized onto a phytosphingosine functionalized gold chip were conducted. The time resolved reflectivity change of a typical SPR spectroscopy experiment is shown in Figure 4.3. An increase in reflectivity was monitored after addition ( $t=0 \mathrm{~s}$ ) of LUVs (POPC/POPE/PIP $, 60: 30: 10, d=100 \mathrm{~nm}$ ) and subsequent immobilization. After rinsing with PBS (Figure 4.3 a) LUVs (POPC/POPE, $60: 40, d=100 \mathrm{~nm}$, Figure $4.3 \mathrm{~b}$ ) were added to the measurement and reference channel. The reflectivity increased and a new plateau was reached. Rinsing with $\mathrm{NaOH}(50 \mathrm{~mm})$ removed reversibly adhering vesicles (Figure $4.3 \mathrm{c}$ ). The measured reflectivity after rinsing with $\mathrm{NaOH}$ was similar to the reflectivity before addition of the vesicles lacking PIP $_{2}$ indicating that no further immobilization of vesicles occurred. Solutions with different concentration of ENTH (Figure $4.3 \mathrm{~d}-\mathrm{k}$ ) were added. The reflectivity increased after ENTH addition indicating binding of the protein to the immobilized LUVs. Rinsing led to a decrease of the reflectivity due 
to dissociation of reversibly bound ENTH. The next concentration of ENTH was added after reaching a plateau.
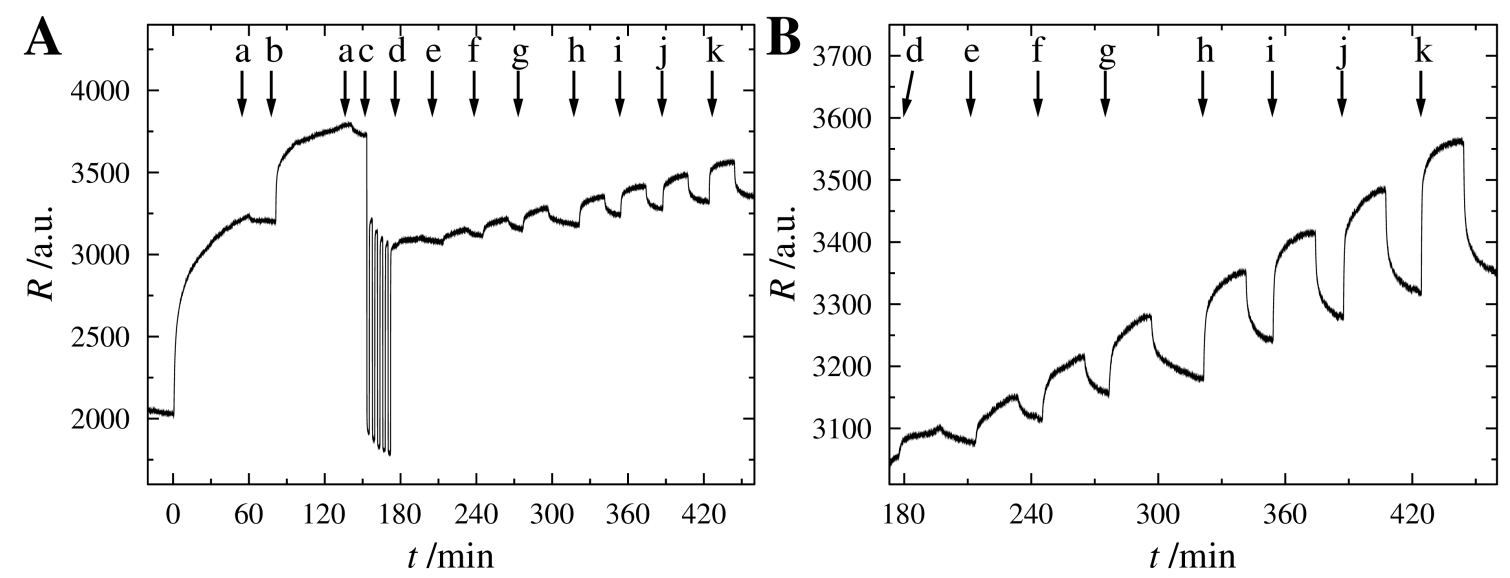

Figure 4.3: A: Representative time resolved reflectivity change obtained by means of SPR spectroscopy. LUVs (POPC/POPE/PIP $2,60: 30: 10, d=100 \mathrm{~nm}$ ) were added at $t=0 \mathrm{~min}$. a: rinsing, b: addition of LUVs (POPC/POPE, 60:40, $d=100 \mathrm{~nm}$ ), c: repeated addition $(5 \times)$ of $\mathrm{NaOH}(50 \mathrm{~mm})$, ENTH addition of d: $50 \mathrm{~nm}, \mathrm{e:} 100 \mathrm{~nm}, \mathrm{f}: 200 \mathrm{~nm}, \mathrm{~g}: 400 \mathrm{~nm}, \mathrm{~h}: 600 \mathrm{~nm}, \mathrm{i}$ : $800 \mathrm{~nm}, \mathrm{j}: 1 \mu \mathrm{M}, \mathrm{k}: 2 \mu \mathrm{M}$. B: Zoom in of A.

Binding of ENTH to curved membranes was analyzed for two different lipid compositions. LUVs composed of POPC/PIP 2 (90:10) were used to compare the dissociation constant of ENTH binding to planar and curved membranes with identical lipid composition. Binding of ENTH to LUVs composed of POPC/POPE/PIP 2 was analyzed as POPE has a small head group which increases the spacing between the lipid head groups which might enhance binding of ENTH to those vesicles. Binding isotherms for both lipid compositions analyzed were obtained by plotting the change in reflectivity caused by binding of ENTH versus the concentration of ENTH (Figure 4.4). Dissociation constants for both lipid compositions were determined by fitting the binding isotherms according to the Langmuir adsorption model using equation 4.1 . Dissociation constants of $0.5 \pm 0.1 \mu \mathrm{M}$ for ENTH binding to LUVs composed of POPC/PIP 2 (90:10) and of $0.69 \pm 0.08 \mu \mathrm{M}$ for binding to LUVs composed of POPC/POPE/PIP 2 (60:30:10) were determined. 


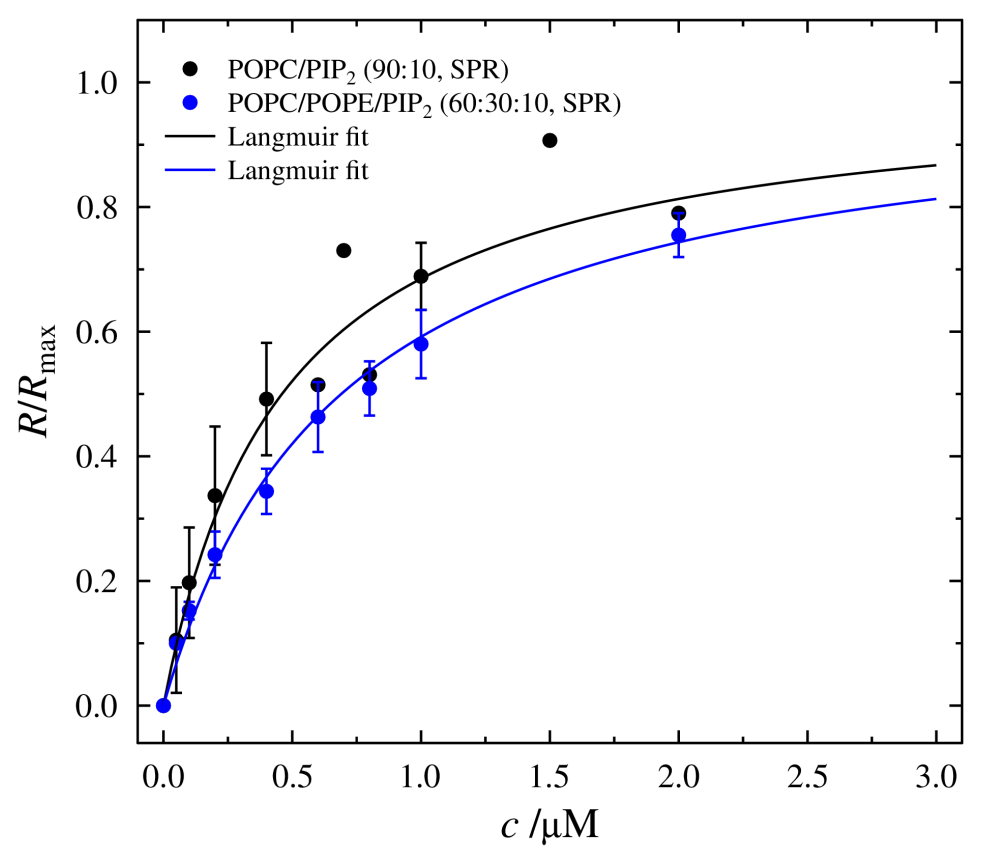

Figure 4.4: Binding isotherms for ENTH binding to LUVs composed of POPC/POPE/PIP 2 (60:30:10, black) and to LUVs composed of POPC/PIP 2 (90:10, blue) in PBS measured by SPR spectroscopy. Isotherms were created from two independent measurements. Errors are the standard deviation of the individual measurements. Data were fitted according to the Langmuir adsorption model using equation 4.1 .

\subsubsection{Average dissociation constant of ENTH binding to $\mathrm{PIP}_{2}$}

The dissociation constant of ENTH binding to a lipid bilayer containing $\mathrm{PIP}_{2}$ was determined by means of RIfS and SPR spectroscopy. For binding to a planar solidsupported membrane (POPC/PIP $2,90: 10$ ) a dissociation constant of $0.8 \pm 0.2 \mu \mathrm{M}$ was determined. When binding to a curved membrane of the same lipid composition a lower dissociation constant of $0.5 \pm 0.1 \mu \mathrm{M}$ was measured. Incorporation of $30 \%$ POPE did not further decrease the dissociation constant but rather increased it to $0.69 \pm 0.08 \mu \mathrm{M}$. Since the errors of the dissociation constants, originating from the uncertainty of the fit of the binding isotherms, overlap a mean dissociation constant of $0.6 \pm 0.1 \mu \mathrm{M}$ was calculated. An ENTH concentration of $3 \mu \mathrm{M}$ was chosen for further experiments as this concentration was reported to result in a maximum tubulation activity ${ }^{[52]}$ At this concentration, assuming a mean dissociation constant of $0.6 \mu \mathrm{M}, 83 \%$ of the $\mathrm{PIP}_{2}$ lipids have bound an ENTH molecule (equation 4.1). 


\subsection{Generation and characterization of nanometer- sized protruded pore-spanning membranes}

Artificial membranes attached to a porous support have been used as a model system to mimic the plasma membrane and the underlying actin cortex. They combine the high stability and planar topology of solid-supported membranes with the lipid dynamics of vesicle based model membranes and are a useful tool to analyze protein-membrane interactions in a defined environment with high statistics. [113, 133-135] It has become evident during the last years that cellular processes are, in addition to the complex interplay of proteins, also regulated by membrane mechanics. Proteins react to changes in membrane tension and as a consequence cellular processes are regulated. [136] Especially, processes involving changes in membrane morphology, such as exo- and endocytosis, are influenced by mechanical properties like membrane tension and bending rigidity. 42, 137] For this reason the mechanics of pore-spanning membranes (PSMs) have been theoretically and experimentally investigated. [120, 138, 140] The free-standing part of pore-spanning membranes is pre-stressed by the adhesion of lipids to the solidsupported areas of the substrate. The adhesion energy of the membrane to the solid support mainly determines the lateral membrane tension and was measured by atomic force microscopy (AFM). 119] 141] AFM allows to locally probe the membrane and to determine membrane tensions with high accuracy. Disadvantages compared to fluorescence microscopy are the need of a physical contact between cantilever and sample and the relatively low time resolution when probing larger areas. Therefore, it would be beneficial to create a model system which allows to use fluorescence microscopy to probe samples fast and non-invasive to obtain information about the mechanical properties of the lipid bilayer.

To produce pore-spanning membranes, giant unilamellar vesicles (GUVs) composed of POPC/Texas Red (99.5:0.5) or DPhPC/Texas Red (99.5:0.5) were added to plasma cleaned porous silicon substrates with a porosity of $2 \%$, a quadratic pore grid of pores with diameters of $850 \mathrm{~nm}$, and pore depths of $10 \mu \mathrm{m}$. Spreading of the vesicles led to the formation of planar pore-spanning membranes (Figure 4.5A) and occurred spontaneously upon contact of the vesicles with the hydrophilic substrate surface. ${ }^{[142]}$ Depending on the GUV size, tens to more than hundred pore-spanning membranes were generated. Quadratically arranged pore-spanning membranes were imaged by confocal fluorescence microscopy (Figure 4.5 A, $x-y$ - 
plane). The fluorescence of the lipid bilayer between the pores was quenched due to the close contact of the membrane to the substrate. The planar topology of the PSMs is shown in the $z$-profile (Figure 4.5 A).

The cylindrical, closed pores were sealed by the pore-spanning membranes. The impermeability of lipid membranes to ions allowed to create an osmolarity gradient by diluting the bulk solution. After dilution of the bulk solution the osmolarity of the buffer inside the pores before dilution $\left(O_{\text {cavity, } 0}\right)$ was higher compared to the buffer outside the pores $\left(O_{\text {bulk }}\right)$, i.e. a positive osmolarity gradient $(\Delta O>0)$ was generated. The applied osmolarity gradient resulted in an influx of water into the cavities which bulged the free-standing membranes from the surface. The diameter of the protruded membranes increased with increasing osmolarity gradient exceeding the pore diameter. Three dimensional imaging of the pore-spanning membranes by confocal laser scanning microscopy (CLSM) after application of an osmolarity gradient resulted in ring shaped structures in $x-y$-plane close to the substrate surface and spherical cap like structures in the $x$ - $z$-plane (Figure 4.5 B-D). A spherical cap geometry was imaged for all osmolarity gradients and is shown schematically in the bottom images of Figure 4.5
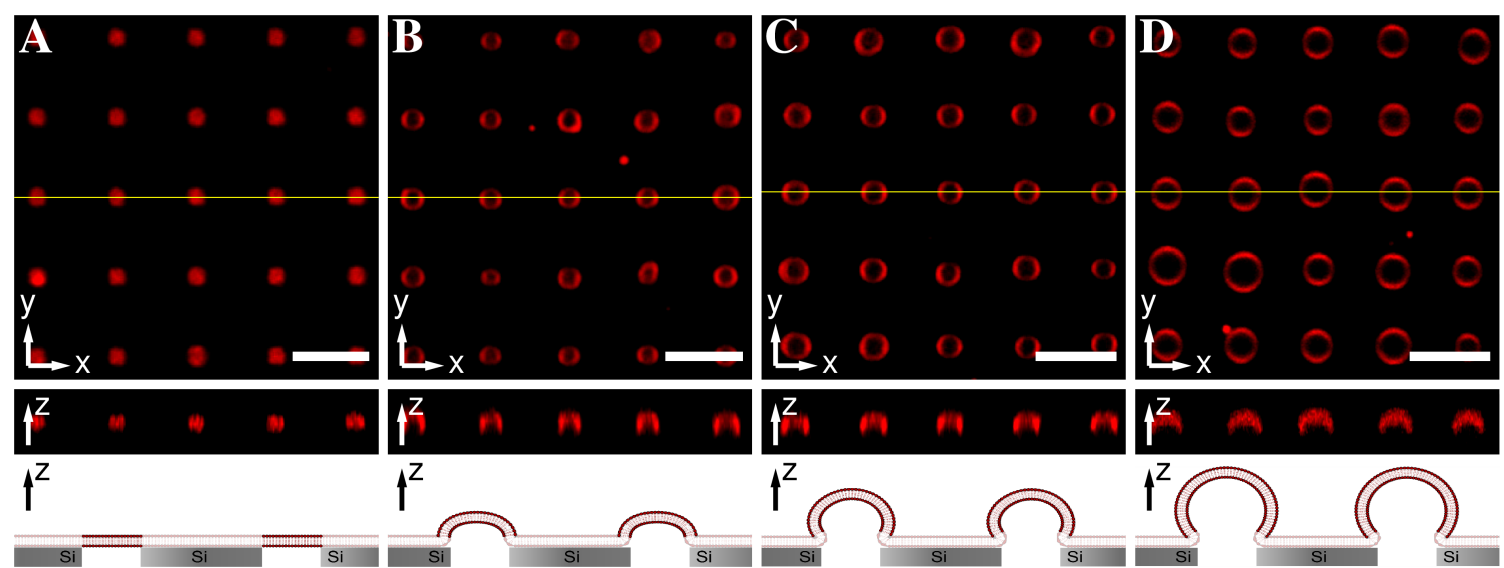

Figure 4.5: Scheme and confocal fluorescence microscopy images of pore-spanning membranes (POPC/Texas Red, 99.5:0.5) on a porous silicon substrate with pore diameters of $850 \mathrm{~nm}$, pore depths of $10 \mu \mathrm{m}$ and a porosity of $2 \%$. The yellow line in the $x$ $y$-plane images indicates the position where $z$-profiles were obtained. The bottom images schematically show the geometry of the protruded PSMs after application of different osmolarity gradients. Osmolarity gradients of A: $0 \mathrm{mOsmol} / \mathrm{L}, \mathbf{B}: 9.5 \mathrm{mOsmol} / \mathrm{L}$, C: $19 \mathrm{mOsmol} / \mathrm{L}$ and D: $29 \mathrm{mOsmol} / \mathrm{L}$ were applied $\left(O_{\text {cavity }, 0}=308 \mathrm{mOsmol} / \mathrm{L}\right)$. Height and diameter of PSMs increase with increasing osmolarity gradient. Height of $z$-profiles: $5 \mu \mathrm{m}$, scale bars: $5 \mu \mathrm{m}$. 
To quantify changes of the membrane morphology after application of an osmolarity gradient CLSM images of protruded pore-spanning membranes (Figure 4.6 A) were thresholded and the corresponding radii of the detected membranes (Figure 4.6 B, yellow contour) were calculated. The average radius of the pore-spanning membranes determined at iso-osmolar conditions was similar to the pore radius whereas the mean membrane radii of the protruded porespanning membranes exceeded the pore radius at higher osmolarity gradients applied. Membrane radii of $0.40 \pm 0.02 \mu \mathrm{m}(n=1285), 0.45 \pm 0.03 \mu \mathrm{m}(n=1806)$, $0.53 \pm 0.02 \mu \mathrm{m}(n=1791), 0.5 \pm 0.05 \mu \mathrm{m}(n=3581)$ and $0.7 \pm 0.1 \mu \mathrm{m}(n=1515)$ were measured for osmolarity gradients of $0 \mathrm{mOsmol} / \mathrm{L}, 4.8 \mathrm{mOsmol} / \mathrm{L}, 9.5 \mathrm{mOsmol} / \mathrm{L}$, $19 \mathrm{mOsmol} / \mathrm{L}$ and $29 \mathrm{mOsmol} / \mathrm{L}$ and are shown in Figure $4.6 \mathrm{C}$.
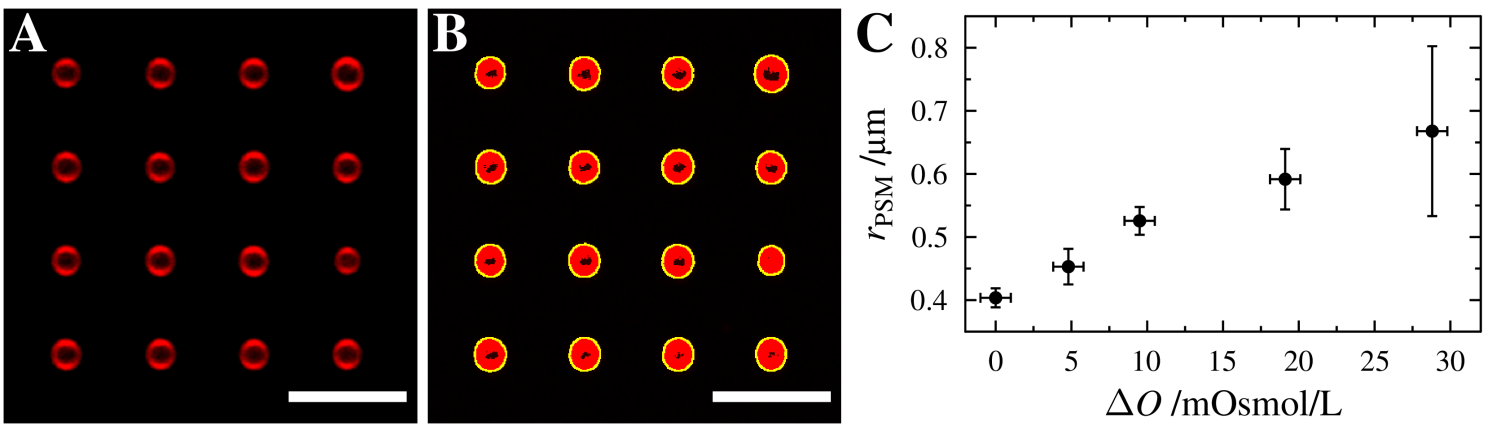

Figure 4.6: A: CLSM image of protruded pore-spanning membranes after application of an osmolarity gradient of $19 \mathrm{mOsmol} / \mathrm{L}$. B: Thresholded image of $\mathbf{A}$ and detected PSMs (yellow contour). C: Radii of pore-spanning membranes measured for different osmolarity gradients from fluorescence microscopy images. Errors of the radii are the standard deviation of the individual measurements. The error of the applied osmolarity gradient is the uncertainty of the osmometric measurements. $O_{\text {cavity }, 0}=308 \mathrm{mOsmol} / \mathrm{L}$, scale bars: $5 \mu \mathrm{m}$.

The fluorescence on the pore rims is quenched to a large extent due to the close contact of the membrane to the substrate. However, since it was not quenched entirely it was possible to image the fluorescence of the lipid bilayer on the pore rims by using a high laser intensity for excitation of the fluorophores and long exposure times. Free-standing membranes were not quenched by the substrate and are therefore overexposed to visualize the membrane on the pore rims. The patch contour is highlighted in green (Figure 4.7A, green contour). 
Protruded pore-spanning membranes have an increased surface area compared to planar membranes. To check how the material for such an increase is provided membrane patches were imaged before and after applying an osmolarity gradient. A large gradient was chosen to be able to image eventual changes of the membrane patch. The membrane patch shrunk laterally after application of an osmolarity gradient of $29 \mathrm{mOsmol} / \mathrm{L}$ and some pore-spanning membranes located at the outer part of the membrane patch ruptured during the protrusion process (Figure 4.7 B, blue contour). The patch shrunk to provide the lipid material required for the formation of protruded pore-spanning membranes with a larger surface area compared to planar PSMs.

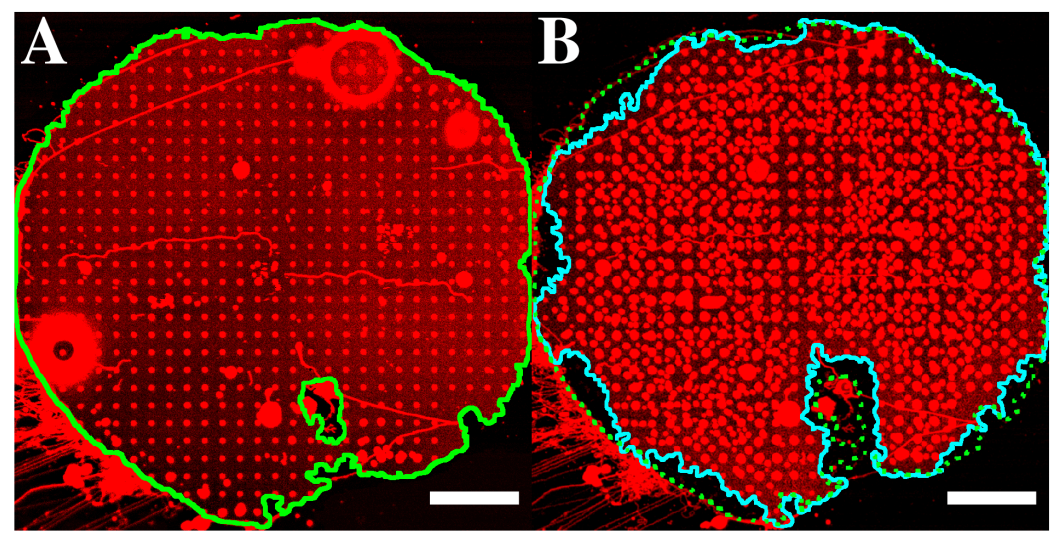

Figure 4.7: Confocal fluorescence microscopy images of a membrane patch (POPC/Texas Red, 99.5:0.5) before (A) and after (B) application of an osmolarity gradient of $29 \mathrm{mOsmol} / \mathrm{L}$. The contour of the patch before and after application of the osmolarity gradient is highlighted in green and blue. $O_{\text {cavity }, 0}=308 \mathrm{mOsmol} / \mathrm{L}$, scale bars: $20 \mu \mathrm{m}$.

Scanning ion conductance microscopy (SICM) experiments were conducted to image the topology of the protruded pore-spanning membranes independently from fluorescence microscopy. SICM allows to probe surface topologies in a contact free manner, has a high axial resolution and is therefore well suited for topography measurements. [143] POPC was replaced by DPhPC due to its higher stability to stably image the protrusions. ${ }^{64}$ A typical SICM image of protruded pore-spanning membranes after application of an osmolarity gradient of $15 \mathrm{mOsmol} / \mathrm{L}$ is shown in Figure 4.8. Regularly arranged protruded pore-spanning membranes with a mean height of $0.6 \pm 0.1 \mu \mathrm{m}(n=64)$, as indicated by the color code, were imaged. A non-covered pore could be clearly distinguished. 


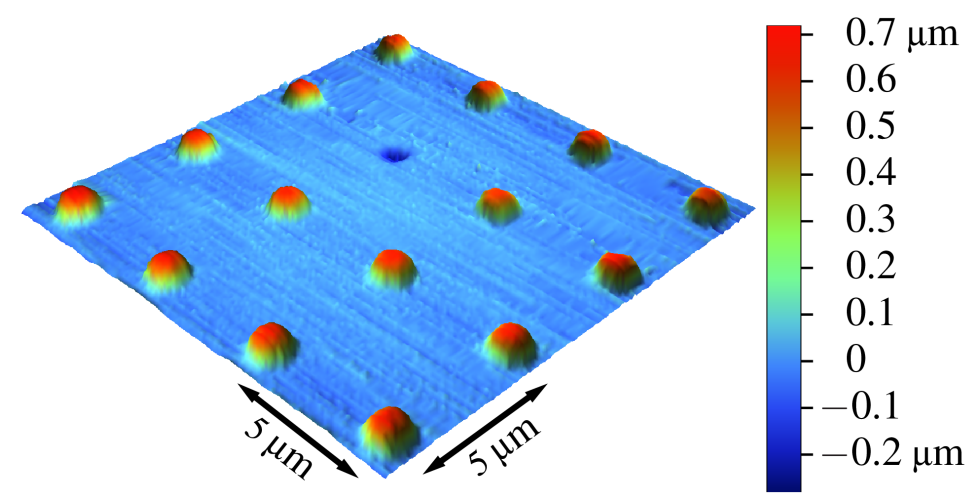

Figure 4.8: Scanning ion conductance microscopy image of protruded pore-spanning $\mathrm{DPhPC}$ membranes after application of an osmolarity gradient of $15 \mathrm{mOsmol} / \mathrm{L}$. Protruded pore-spanning membranes were imaged as bulges as indicated by the color code. A noncovered pore was imaged indicated by the dark blue color.

To further quantify the morphological changes of pore-spanning membranes after application of an osmolarity gradient the height of the protruded pore-spanning membranes was measured by CLSM and SICM, evaluated and correlated to the applied osmolarity gradient. Axial intensity profiles, one in the center of each PSM (Figure 4.9 A, blue) and four centered along the diagonal between the neighbored pores (Figure 4.9A, green), were measured in $z$-direction and fitted with a Gaussian function (Figure 4.9 B).
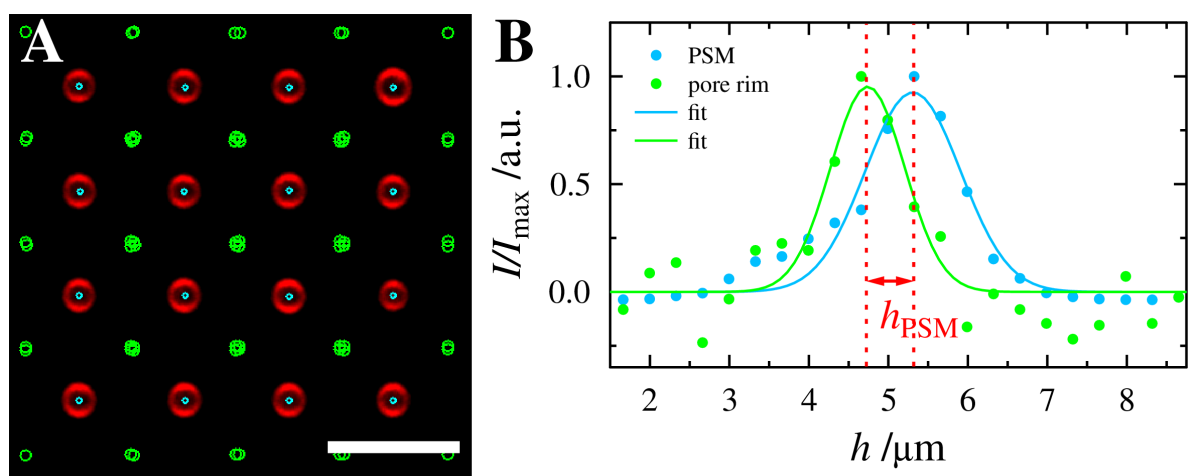

Figure 4.9: A: CLSM image of pore-spanning membranes (POPC/Texas Red, 99.5:0.5, red) after application of an osmolarity gradient of $19 \mathrm{mOsmol} / \mathrm{L}\left(O_{\text {cavity }, 0}=308 \mathrm{mOsmol} / \mathrm{L}\right)$ with regions for measuring intensity profiles of the protruded pore-spanning membranes (blue) and the membrane located on the pore rim (green). B: Representative normalized fluorescence intensity profiles of a protruded pore-spanning membrane and the membrane located on the pore rim. Scale bar: $5 \mu \mathrm{m}$. 
As all the regions of interest (ROIs) on the pore rim used for measuring the intensity profiles had identical distances to the ROI in the center of the pore-spanning membrane, the determined absolute heights of the membrane on the pore rim were averaged to correct for a possible tilting of the substrate. The height of the pore-spanning membrane was obtained by subtracting the averaged absolute rim height from the absolute height of the pore-spanning membrane. Heights of pore-spanning membranes were plotted and fitted as a function of the applied osmolarity gradient. The lateral membrane tension was then extracted as described below.

According to Young-Laplace the equilibrium osmotic pressure difference $\Delta \Pi_{\mathrm{eq}}$ is equal to the ratio of twice the tension $\sigma$ to the Laplace radius $r_{\text {Laplace }}$ (equation 4.2).

$$
\Delta \Pi_{\mathrm{eq}}=\frac{2 \sigma}{r_{\text {Laplace }}}
$$

This theory is valid for spheres with an interface of zero thickness. It can be applied since the protruded membranes have a spherical cap geometry and since the thickness is very small compared to the radius and can therefore be neglected. [144] Planar pore-spanning membranes were formed at iso-osmolar conditions. The osmotic pressure of the bulk solution $\left(\Pi_{\text {bulk }}\right)$ is then equal to the osmotic pressure inside the pores $\left(\Pi_{\text {cavity, } 0}\right)$. Lowering the osmolarity of the bulk solution by dilution with water led to an influx of water into the cavities. The membranes protruded from the surface, formed a spherical cap with the height $h$ and the volume inside the pore $\left(V_{\text {cavity }}\right)$ increased by $\Delta V_{\text {cavity. }}$. The resulting equilibrium osmotic pressure difference is then calculated as shown by equation 4.3 .

$$
\Delta \Pi_{\mathrm{eq}}=\frac{V_{\text {cavity }}}{V_{\text {cavity }}+\Delta V_{\text {cavity }}} \Pi_{\text {cavity }, 0}-\Pi_{\text {bulk }} \text {. }
$$

The Laplace radius is calculated by rearranging geometric relationships of a spherical cap (equation 4.4 ) to be:

$$
r_{\text {Laplace }}=\frac{r_{\text {pore }}^{2}+h^{2}}{2 h}
$$

As the planar pore-spanning membranes adopt to a spherical cap geometry upon protrusion the increase in pore volume $\left(\Delta V_{\text {cavity }}\right)$ is equal to the volume of the spherical cap $\left(V_{\text {cap }}\right)$. The radius of the base of the cap is fixed by the pore radius 
$\left(r_{\text {pore }}\right)$. The volume of a spherical cap $\left(V_{\text {cap }}\right)$ with the height $h$ is calculated as shown by equation 4.5 .

$$
V_{\text {cavity }}=V_{\text {cap }}=\frac{\pi h}{6}\left(3 r_{\text {pore }}^{2}+h^{2}\right) \text {. }
$$

Combination of equation 4.2 and 4.5 leads to equation 4.6

$$
\frac{2 \sigma}{\frac{r_{\text {pore }}^{2}+h^{2}}{2 h}}=\frac{V_{\text {cavity }}}{V_{\text {cavity }}+\frac{\pi h}{6}\left(3 r_{\text {pore }}^{2}+h^{2}\right)} \Pi_{\text {cavity, } 0}-\Pi_{\text {bulk }}
$$

which can be rearranged to equation 4.7

$$
\Pi_{\text {bulk }}=-\frac{4 \sigma h}{r_{\text {pore }}^{2}+h^{2}}+\frac{V_{\text {cavity }}}{V_{\text {cavity }}+\frac{\pi h}{6}\left(3 r_{\text {pore }}^{2}+h^{2}\right)} \Pi_{\text {cavity }, 0} .
$$

Equation 4.7 is only dependent on known or measured parameters and on the lateral membrane tension. Fitting it to the data yielded the membrane tension (Figure $4.10 \mathrm{~A}$ ). To assess how different membrane tensions would change the height of the membrane protrusions theoretically expected heights of protruded pore-spanning membranes were simulated for membrane tensions of $0.02 \mathrm{mN} / \mathrm{m}$, $0.2 \mathrm{mN} / \mathrm{m}, 2 \mathrm{mN} / \mathrm{m}$, and $5 \mathrm{mN} / \mathrm{m}$. However, since the osmolality and not the osmotic pressure was experimentally measured the height of the pore-spanning membranes was correlated with the applied osmolarity gradient. A relation between osmolarity gradient and osmotic pressure difference is given by equation 4.8 with $R$ being the universal gas constant $\left(R=8.314 \mathrm{~J} \mathrm{~mol}^{-1} \mathrm{~K}^{-1}\right)$ and $\mathrm{T}$ being the absolute temperature $(T=293 \mathrm{~K})$.

$$
\Delta O=\frac{\Delta \Pi}{R T}=\frac{\Pi_{\text {cavity }, 0}-\Pi_{\text {bulk }}}{R T}
$$

Heights of protruded pore-spanning membranes after application of different osmolarity gradients were measured by CLSM and SICM and are shown in Figure 4.10 B. Fluorescence microscopy data and SICM data match within the error range. Increasing heights of the protruded pore-spanning membranes were measured with increasing osmolarity gradient. Large membrane tensions result in small protrusion heights as the height of the protruded pore-spanning membranes is governed by the lateral membrane tension. Especially for large osmolarity gradients membrane heights were measured corresponding to larger membrane tensions in the range of $5 \mathrm{mN} / \mathrm{m}$. This could be caused by deviations of the 
membrane shape from the assumed ideal spherical cap geometry.

Fitting of equation 4.7 to the data obtained by CLSM and SICM yields a membrane tension of $1.4 \pm 0.7 \mathrm{mN} / \mathrm{m}$ (Figure 4.10 , dashed lines) which is in the same range as the membrane tension of planar pore-spanning membranes generated on open pores of a hydrophilically functionalized substrate measured by means of atomic force microscopy. 119,139
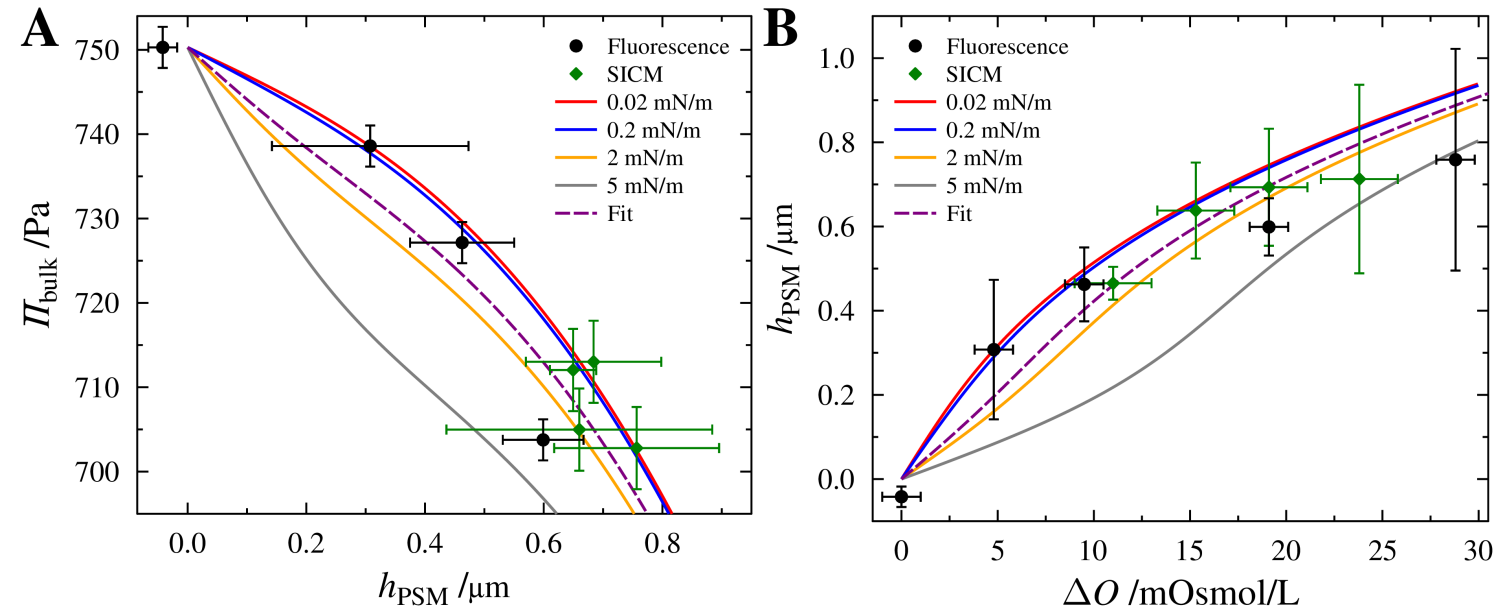

Figure 4.10: A: Osmotic pressure of the bulk solution calculated from the applied osmolarity gradient and corresponding measured heights of protruded pore-spanning POPC and DPhPC membranes. B: Heights of protruded pore-spanning POPC and DPhPC membranes measured for different osmolarity gradients. Theoretical heights of protruded pore-spanning membranes were simulated for different lateral tensions as a function of the osmotic pressure (A) and applied osmolarity gradient (B). Errors of membrane height are the standard deviation of the Gaussian distribution and errors of the applied osmolarity gradient are the uncertainty of the osmometric measurements. Lipid compositions: POPC (CLSM, black), DPhPC (SICM, green). $O_{\text {cavity }, 0}=308 \mathrm{mOsmol} / \mathrm{L}$.

\subsection{Interaction of ENTH with nanometer-sized protruded pore-spanning membranes}

The ENTH domain of the endocytotic protein epsin is an evolutionary conserved domain present in different endocytotic proteins. It binds specifically to the receptor lipid $\mathrm{PIP}_{2}$ and is believed to sense and induce membrane curvature. 13, 44, 145] Fluorescently labeled ENTH-Alexa488 $\left(c_{\text {ENTH }}=3 \mu \mathrm{M}\right)$ has been added to protruded PSMs of different compositions (DPhPC/PIP $2 /$ Texas Red, 98.5:1:0.5; 
POPC/Chol/PIP $2 /$ Texas Red, 78.5:20:1:0.5; POPC/PIP $2 /$ Texas Red, 98.5:1:0.5) which contained $1 \%$ of the receptor lipid $\mathrm{PIP}_{2}$. The pore-spanning membranes were precurved by applying an osmolarity gradient of $19 \mathrm{mOsmol} / \mathrm{L}$ to provide a homogeneous scaffold with low curvature. A representative time series of protruded pore-spanning membranes composed of DPhPC (DPhPC/PIP $2 /$ Texas Red, 98.5:1:0.5) is shown in Figure 4.11. An increase of the green fluorescence of the labeled protein was detected (Figure $4.11 \mathrm{~B})$ after addition of ENTH $\left(c_{\text {ENTH }}=3 \mu \mathrm{M}\right)$ colocalizing with the Texas Red fluorescence (Figure 4.11 A) which indicates binding of ENTH to the protruded pore-spanning membranes.
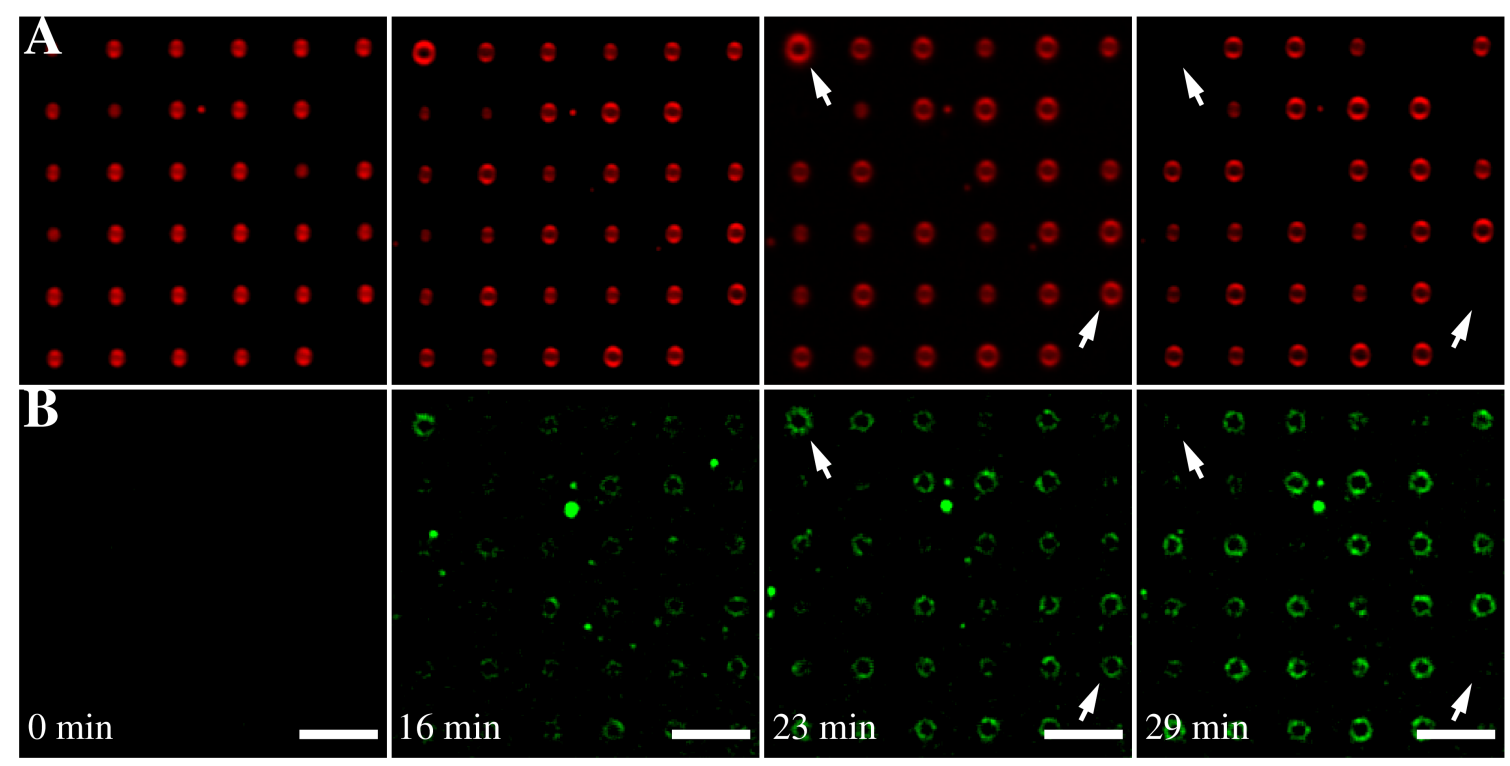

Figure 4.11: Time series of confocal fluorescence microscopy images of protruded porespanning membranes (DPhPC/PIP $2 /$ Texas Red, 98.5:1:0.5, $\Delta O=19 \mathrm{mOsmol} / \mathrm{L}$ ). Intensity of the ENTH-Alexa488 fluorescence $\left(c_{\mathrm{ENTH}}=3 \mu \mathrm{M}\right.$, green, B $)$ increases as a function of time and colocalizes with the Texas Red fluorescence (A). Growth of the PSMs and disappearance of individual PSMs was observed (positions marked at $t=23 \mathrm{~min}$ and $t=29 \mathrm{~min}$ ). ENTH was added at $=1 \mathrm{~min}$. $O_{\text {cavity }, 0}=322 \mathrm{mOsmol} / \mathrm{L}$, scale bars: $5 \mu \mathrm{m}$.

Quantification of the membrane radii revealed a slight decrease of the average membrane radius as a function of time for all lipid compositions when $\mathrm{PIP}_{2}$ was absent (Figure 4.12 A1-C1). This is attributed to evaporation of water which increased the osmolarity of the bulk solution and therewith reducing the osmolarity gradient. The number of membranes remained almost constant during the observation windows of 30-40 min (Figure 4.12 A2-C2) showing the long-term stability of the system. 
The radius of DPhPC membranes containing $1 \% \mathrm{PIP}_{2}$ increased upon ENTH addition as a function of time (Figure 4.12 A1). As shown in Figure 4.11 some PSMs disappeared after a certain period of time. Quantification revealed a decrease of the number of membranes present in the field of observation after a lag phase of approximately $18 \mathrm{~min}$ (Figure 4.12 A2). To use a physiologically more relevant lipid the experiment was repeated with DPhPC being replaced by POPC which represents a major component of the plasma membrane. [58, 146] Growth of the protruded pore-spanning membranes composed of POPC/ $\mathrm{PIP}_{2} /$ Texas Red (98.5:1:0.5) after addition of ENTH was barely detectable (Figure 4.12 B1) but instead the membranes started to disappear directly after ENTH addition with almost no lag phase. All pore-spanning membranes disappeared within the observation time window of 30-40 min (Figure 4.12 B2).

Since unspecific interactions were excluded by RIfS (Section 4.1.1) and DPhPC bilayers grew after ENTH addition as a function of time, the reduced mechanical stability of POPC compared to DPhPC could explain the observed disappearance of the POPC membranes after ENTH addition. The fatty acid chains of DPhPC are branched by methyl groups. Thereby, DPhPC forms intertwined networks resulting in a high mechanical stability as the networks need to be broken when deforming the membrane. 61,62] To check whether the reduced stability of POPC compared to DPhPC is the reason for the preferred disappearance of the POPC membranes after ENTH binding, the experiment was repeated with POPC membranes containing $20 \%$ cholesterol. Cholesterol has been shown to increase rupture tension and area compressibility modulus of POPC bilayers and thereby to enhance the membrane stability. $\left.{ }^{[64}\right]$ Indeed, growing of the POPC/Chol membranes containing $20 \%$ cholesterol (POPC/Chol/PIP $2,79: 20: 1$ ) was observed after addition of ENTH $\left(c_{\mathrm{ENTH}}=3 \mu \mathrm{M}\right.$, Figure 4.12 $\left.\mathrm{C} 1\right)$. Additionally, disappearance of POPC/Chol membranes started earlier compared to DPhPC membranes but later than observed for pure POPC membranes (Figure 4.12 C2) supporting the higher stability of the POPC/Chol mixture compared to pure POPC membranes. The ENTH domain of epsin is involved in clathrin mediated endocytosis and has been shown to cause the formation of highly curved structures such as vesicles and tubes. $\left.{ }^{8,}, 13,44\right]$ To check whether the disappearance of membranes after ENTH binding can be attributed to ENTH's vesiculation or tubulation activity 3D spinning disc confocal laser microscopy (SDCLM) measurements were conducted. 

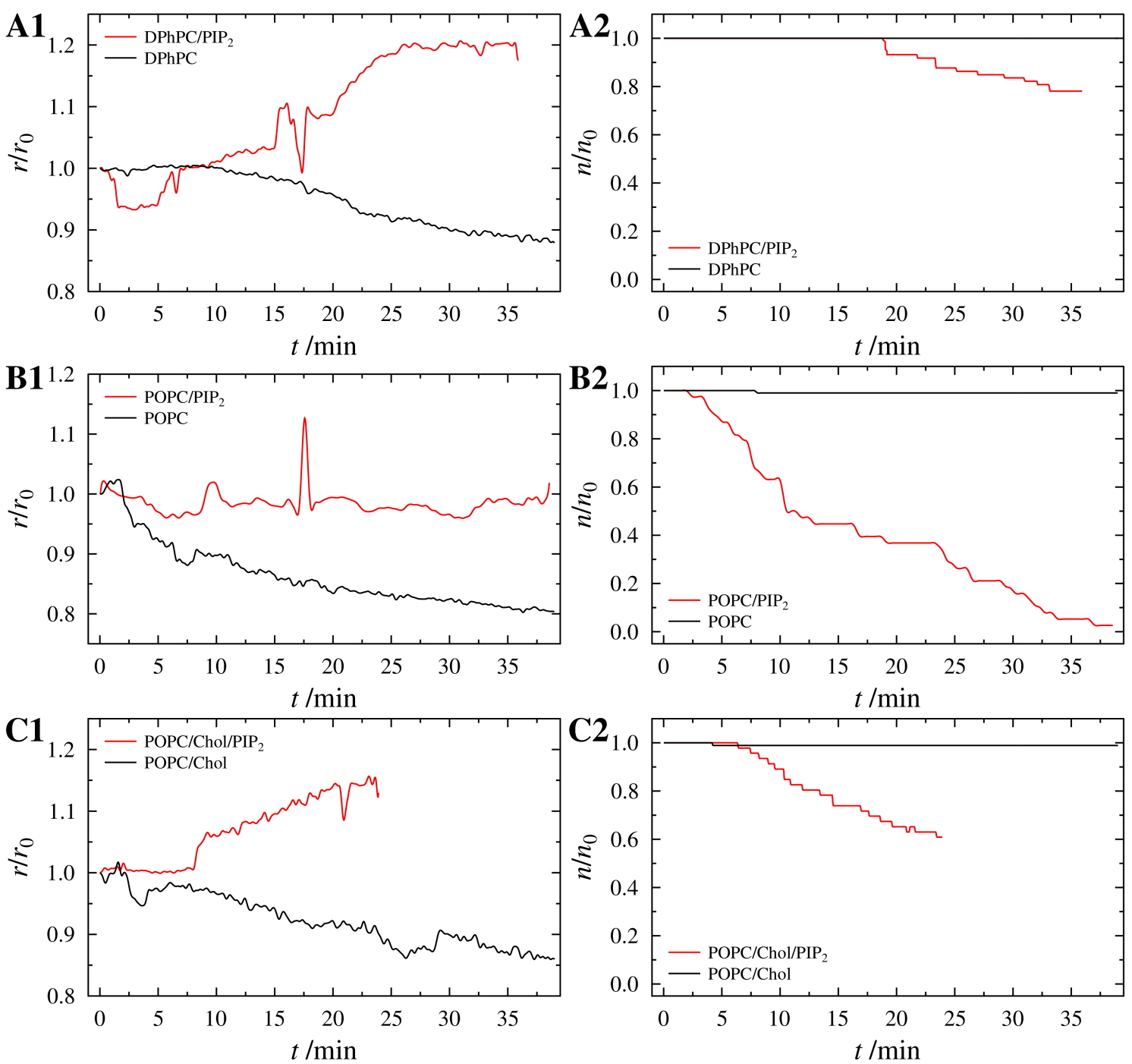

Figure 4.12: Normalized mean radius and number of pore-spanning membranes with (red) and without $\mathrm{PIP}_{2}$ (black) as a function of time. The mean radius of $\mathrm{PIP}_{2}$-containing DPhPC (A1, DPhPC/PIP $\left.2,99: 1, r_{0}=0.57 \mu \mathrm{m}, n_{0}=73\right)$ and POPC/Chol (C1, POPC/Chol $/ \mathrm{PIP}_{2}$, 79:20:1, $\left.r_{0}=0.49 \mu \mathrm{m}, n_{0}=45\right)$ membranes increases as a function of time after ENTH addition $\left(c_{\mathrm{ENTH}}=3 \mu \mathrm{M}\right)$ whereas no increase is monitored for POPC $\left(\mathbf{B} 1, \mathrm{POPC} / \mathrm{PIP}_{2}\right.$, 99:1, $\left.r_{0}=0.51 \mu \mathrm{m}, n_{0}=38\right)$ membranes. For $\mathrm{PIP}_{2}$-free membranes a slight decrease of the mean radius is observed (DPhPC: $r_{0}=0.68 \mu \mathrm{m}, n_{0}=219$, POPC: $r_{0}=0.49 \mu \mathrm{m}$, $n_{0}=103$, POPC/Chol (80:20): $\left.r_{0}=0.64 \mu \mathrm{m}, n_{0}=92\right)$. A2, B2, C2: Normalized number of pore-spanning membranes with (red) and without $\mathrm{PIP}_{2}$ (black) as a function of time. Disappearance of pore-spanning membranes only occurs after ENTH addition and for bilayers containing $\mathrm{PIP}_{2}$. $\mathrm{PIP}_{2}$-free membranes do not react to ENTH addition. $O_{\text {cavity }, 0}=322 \mathrm{mOsmol} / \mathrm{L}, \Delta O=19 \mathrm{mOsmol} / \mathrm{L}$. 
SDCLM enables image acquisition with short exposure times (typically a few milliseconds) and allows to obtain high frame rates. Figure 4.13 shows a time series of $3 \mathrm{D}$ reconstructions of pore-spanning membranes (DPhPC/PIP $2 /$ Texas Red, 98.5:1:0.5) imaged with nine $z$-stacks per second (each 21 planes, $\Delta z=0.35 \mu \mathrm{m}$ ) after ENTH addition $\left(c_{\mathrm{ENTH}}=3 \mu \mathrm{M}\right)$. These parameters ensured a frame rate which is fast enough to detect vesiculation or tubulation caused by ENTH binding.
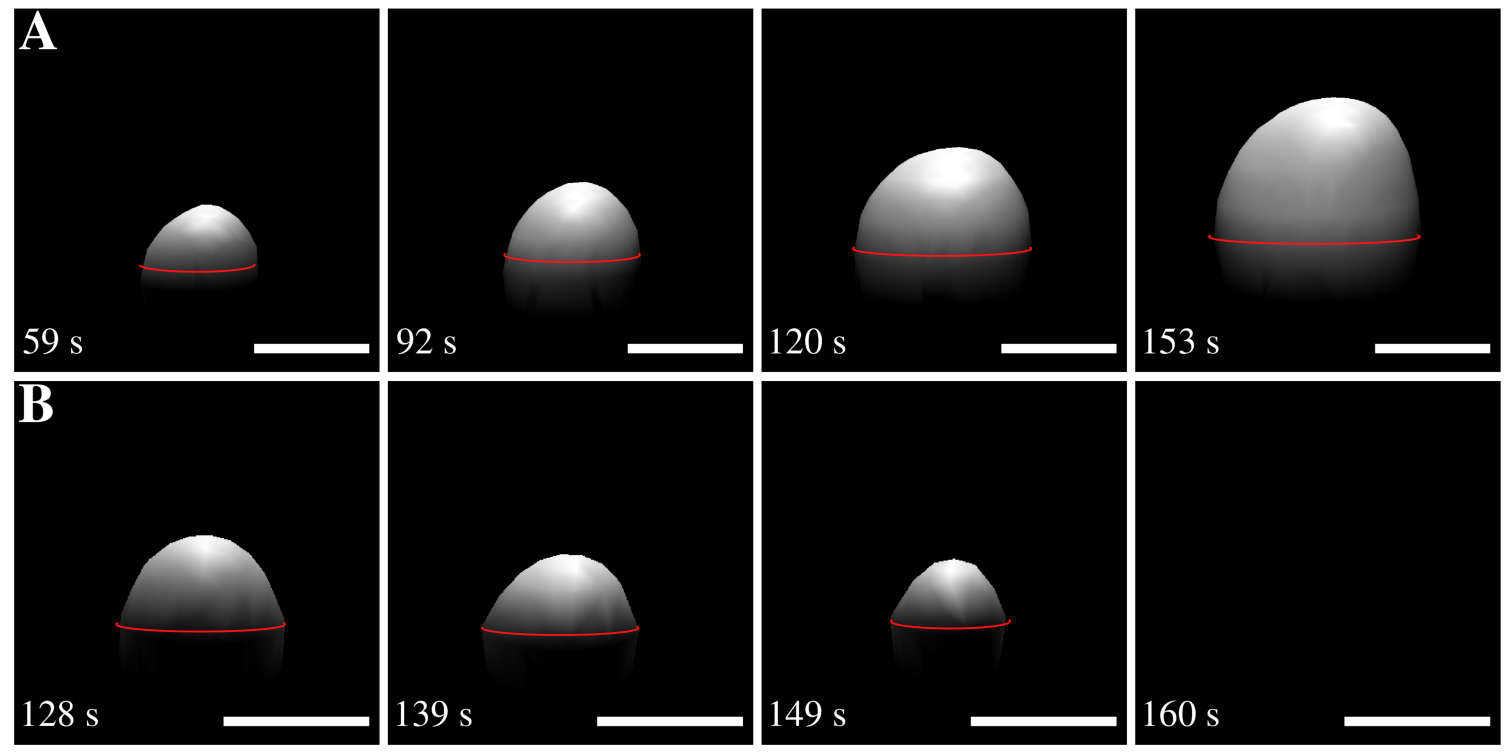

Figure 4.13: Surface rendering of SDCLM images of growing (A) and shrinking (B) protruded PSMs (DPhPC/PIP $2 /$ Texas Red, 98.5:1:0.5) after application of an osmolarity gradient of $19 \mathrm{mOsmol} / \mathrm{L}$ and subsequent addition of ENTH $\left(c_{\mathrm{ENTH}}=3 \mu \mathrm{M}\right)$ as a function of time. ENTH was added at $t=0 \mathrm{~s}$. $O_{\text {cavity }, 0}=290 \mathrm{mOsmol} / \mathrm{L}$, scale bars: $1 \mu \mathrm{m}$.

Growth (Figure 4.13A) and shrinkage (Figure 4.13B) of protruded membranes was observed as a function of time after ENTH addition. The position of the substrate is marked by a red line as the fluorescence of the membrane at the pore rim is quenched. The frame rate was fast enough to image detachment of vesicles which was proven by the imaging of lipid material being transported through the field of view. Even though the possibility to detect detaching vesicles was proven, no vesiculation or tubulation was observed after ENTH addition. A possible explanation for the disappearance of individual PSMs after ENTH addition as observed by CLSM and SDCLM is a break down of the osmolarity gradient caused by the formation of membrane defects upon insertion of the ENTH helix into the lipid bilayer when binding to $\mathrm{PIP}_{2} \cdot 47,147$ These defects have shown to increase 
with positive curvature and would explain the preferential binding of ENTH to curved membranes. 148, 149]

The height of protruded pore-spanning membranes is governed by the lateral membrane tension. Therefore, growing of the protrusions might be caused by a local decrease in membrane tension. Mechanically, a reduced membrane tension would lower the energy required for the formation of curvature and could facilitate the formation of vesicles as observed in vivo after ENTH addition. [13] Unfortunately, it was not possible to locate the lipid material after disappearance due to fluorescence quenching of the substrate. As the porous substrates used for the experiments have pore radii of $425 \mathrm{~nm}$, which is close to the optical limit of the microscope used, substrates with larger pores were used to minimize uncertainties from the determination of the height of the pore-spanning membranes.

\subsection{Generation and characterization of micrometer- sized protruded pore-spanning membranes}

\subsubsection{Influence of the substrate dimension on the determination of the lateral membrane tension}

Over the last years, it became evident that cells regulate processes such as endoand exocytosis or cell movement not only by the complex interplay of proteinprotein and protein-membrane interactions but also by changing their membrane tension. 42, 137 The applicability of protruded pore-spanning membranes as a model system with tunable curvature for the analysis of membrane-protein interactions has been shown (Section 4.3). Binding of ENTH to $\mathrm{PIP}_{2}$-containing membranes was imaged by fluorescence microscopy and the growth of protruded pore-spanning membranes has been attributed to a local decrease of the lateral membrane tension. [150]

Membrane tension of protruded pore-spanning membranes is calculated applying a Young-Laplace behavior as a function of the applied osmolarity gradient and the geometry of pores and protruded pore-spanning membranes. The height of protruded pore-spanning membranes on substrates with nanometer-sized pores has been measured and a lateral membrane tension of $1.4 \pm 0.7 \mathrm{mN} / \mathrm{m}$ 
was calculated (Section 4.2). A large distribution of the heights of protruded pore-spanning membranes on nanometer-sized pores after application of large osmolarity gradients has been measured (Figure 4.10). This might be caused by a breakdown of the osmolarity gradient between bulk solution and pore interior or by deviations in shape of the protruded pore-spanning membranes from the ideal spherical cap geometry which was used for the calculation of the lateral membrane tension. Therefore, the aim was to create a "tension sensor" of protruded porespanning membranes which do not exceed the pore radius in height or radius to improve the accuracy of the "tension sensor". The constrain of a maximum height of the protruded pore-spanning membranes equal to pore radius ensures a spherical cap geometry. The osmolarity gradient required to protrude a planar porespanning membrane until its height is equal to the pore radius was relatively small $(\approx 10 \mathrm{mOsmol} / \mathrm{L})$ for the porous substrates used. A breakdown of the osmolarity gradient between pore interior and bulk solution is therefore unlikely.

The changes in membrane height resulting from different membrane tensions depend on the applied osmolarity gradient $(\Delta O)$, the absolute osmolarity of the buffer used during spreading of the GUVs $\left(O_{\text {cavity, } 0}\right)$ and the geometry of the pores. Porous substrates were available with a pore depth of $8 \mu \mathrm{m}$ and with pore radii of $1.75 \mu \mathrm{m}, 2.25 \mu \mathrm{m}$ and $2.75 \mu \mathrm{m}$. To evaluate the optimal pore geometry, to be able to determine the lateral membrane tension of protruded pore-spanning membranes with high accuracy, the theoretical membrane height of pore-spanning membranes on pores with a radius of $1.75 \mu \mathrm{m}$ (Figure 4.14 A), $2.25 \mu \mathrm{m}$ (Figure $4.14 \mathrm{~B}$ ) and $2.75 \mu \mathrm{m}$ (Figure $4.14 \mathrm{C}$ ) was simulated for three different lateral tensions, a pore depth of $8 \mu \mathrm{m}$ and an osmolarity during PSM formation of $O_{\text {cavity }, 0}=100 \mathrm{mOsmol} / \mathrm{L}$. The height of the protruded pore-spanning membranes was experimentally measured by imaging of $z$-stacks of PSMs by means of confocal fluorescence microscopy. Since conventional confocal fluorescence microscopy has a finite axial resolution, typically in the range of $500 \mathrm{~nm}$, larger heights of the pore-spanning membranes would result in a higher accuracy of the determination of the lateral membrane tension. 151, 152] Otherwise small deviations in membrane height would result in large relative errors of the PSM height. Even though the relative error of the protruded pore-spanning membranes is reduced by large pore radii, differences in membrane height, originating from different lateral tensions, decrease with increasing pore radius. This reduces the accuracy of the determination of the lateral membrane tension as small deviations 
in membrane height translate into large changes in lateral membrane tension. Therefore, pores with a radius of $1.75 \mu \mathrm{m}$ were selected as they have the broadest spacing of the membrane heights simulated for three different membrane tensions (Figure $4.14 \mathrm{~A}-\mathrm{C}$ ).
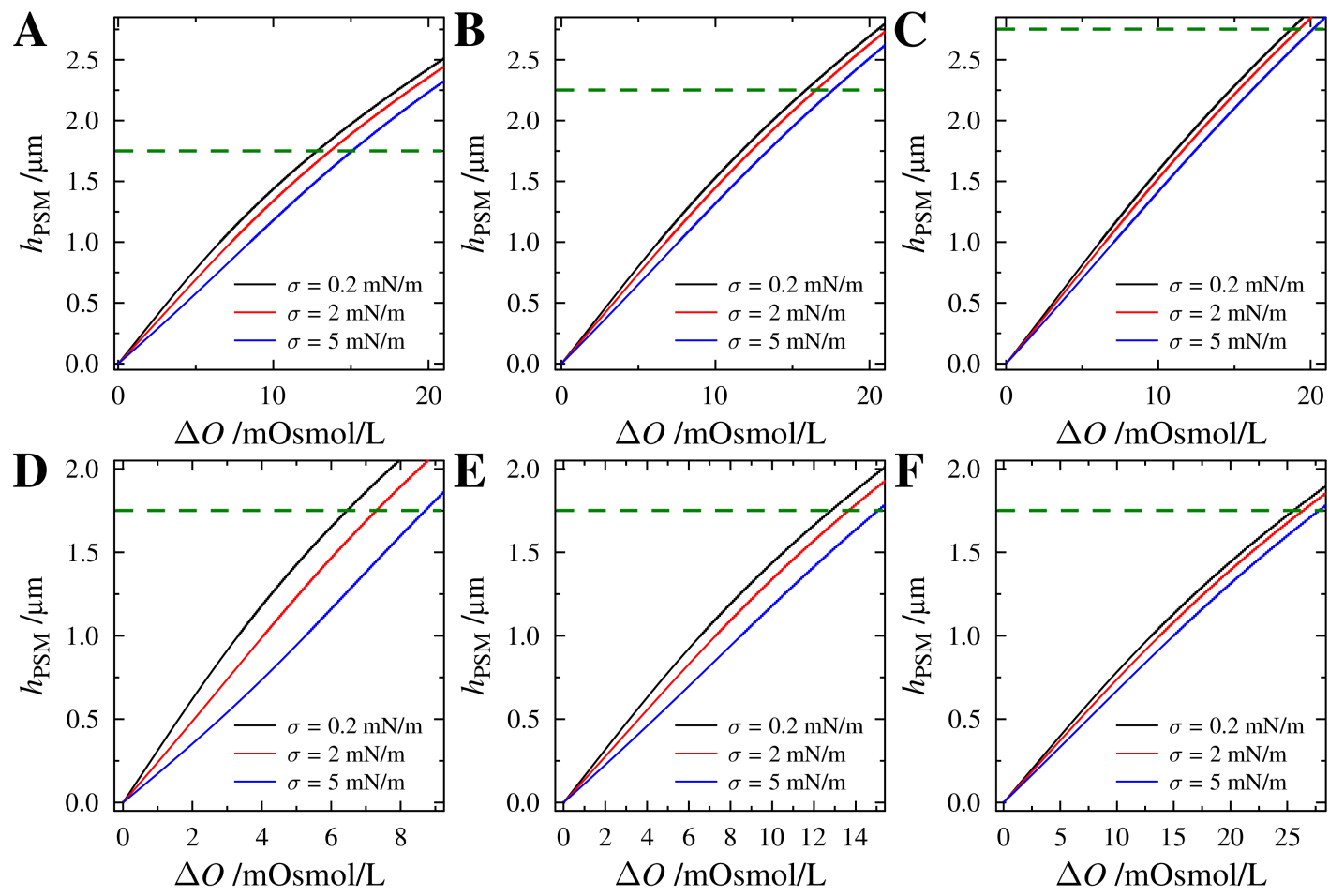

Figure 4.14: Calculated height of pore-spanning membranes on pores with a depth of $8 \mu \mathrm{m}$ as a function of the applied osmolarity gradient, simulated for three different membrane tensions $(0.2 \mathrm{mN} / \mathrm{m}, 2 \mathrm{mN} / \mathrm{m}$ and $5 \mathrm{mN} / \mathrm{m})$ and osmolarities used for PSM formation. A-C: Heights of PSMs simulated for pore radii of $1.75 \mu \mathrm{m}(\mathbf{A}), 2.25 \mu \mathrm{m}(\mathbf{B})$ and $2.75 \mu \mathrm{m}(\mathbf{C})$, a pore depth of $8 \mu \mathrm{m}$ and an osmolarity inside the pore during spreading of the GUVs of $O_{\text {cavity }, 0}=100 \mathrm{mOsmol} / \mathrm{L}$. The separation of the membrane heights resulting from different lateral tensions decreases with increasing pore radius. D-F: Heights of PSMs simulated for a pore radius of $1.75 \mu \mathrm{m}$ and different osmolarities during spreading of the GUVs of $O_{\text {cavity }, 0}=50 \mathrm{mOsmol} / \mathrm{L}(\mathbf{D}), O_{\text {cavity }, 0}=100 \mathrm{mOsmol} / \mathrm{L}(\mathbf{E})$ and $O_{\text {cavity }, 0}=200 \mathrm{mOsmol} / \mathrm{L}(\mathbf{F})$. The separation of the membrane heights resulting from different lateral tensions decreases with increasing osmolarity during spreading of the GUVs. The height of the PSMs equal to pore radius is marked by the dotted green line.

The influence of the osmolarity inside the pores during spreading of GUVs and the formation of pore-spanning membranes for the selected pore geometry with pore radii of $1.75 \mu \mathrm{m}$ and a pore depth of $8 \mu \mathrm{m}$ was then analyzed (Figure $4.14 \mathrm{D}-\mathrm{F}$ ). Simulating the heights of protruded pore-spanning membranes with three different 
lateral membrane tensions and osmolarities during GUV spreading $\left(O_{\text {cavity, } 0}\right.$ : $50 \mathrm{mOsmol} / \mathrm{L}, 100 \mathrm{mOsmol} / \mathrm{L}$ and $200 \mathrm{mOsmol} / \mathrm{L}$ ) revealed that the spacing of the membrane height resulting from different lateral membrane tensions decreases with increasing osmolarity inside the pores (Figure $4.14 \mathrm{D}-\mathrm{F}$ ). The largest spacings of membrane heights originating from different membrane tensions were calculated for an osmolarity during PSM formation of $O_{\text {cavity, } 0}=50 \mathrm{mOsmol} / \mathrm{L}$. However, the critical osmolarity gradient when the height of the protruded pore-spanning membrane equals the pore radius decreases with decreasing pore radius. Since the height of the pore-spanning membrane is non-linearly dependent on the applied osmolarity gradient and the lateral membrane tension (equation 4.7), a preferentially large number of data points is required for an accurate fitting of the data yielding the lateral membrane tension. A minimum of five data points was defined to be required for fitting the data. A minimal separation of $2 \mathrm{mOsmol} / \mathrm{L}$ between two data points was chosen as the osmolarity could be measured with an accuracy of $\pm 1 \mathrm{mOsmol} / \mathrm{L}$. Therefore, to be able to fit equation 4.7 to minimal five osmolarity gradients the height of the pore-spanning membrane must not exceed the pore radius when applying an osmolarity gradient of $10 \mathrm{mOsmol} / \mathrm{L}$.

For an osmolarity of $50 \mathrm{mOsmol} / \mathrm{L}$ protruded pore-spanning membranes exceed the pore radius in height when applying osmolarity gradients larger than 7 to $8 \mathrm{mOsmol} / \mathrm{L}$ preventing to measure the previously defined minimum of five osmolarity gradients with a spacing of $2 \mathrm{mOsmol} / \mathrm{L}$. Additionally, small uncertainties of the osmolarity gradient would result in large changes of membrane height further decreasing the accuracy of the determined lateral tension. Therefore, an osmolarity of $100 \mathrm{mOsmol} / \mathrm{L}$ was chosen for PSM formation to achieve a compromise between highest sensitivity and minimized uncertainties from the determination of osmolarities or protrusion heights.

\subsubsection{Height and lateral tension of micrometer-sized protruded pore-spanning membranes}

Porous substrates with pore diameters of $3.5 \mu \mathrm{m}$, pore heights of $8 \mu \mathrm{m}$ and a porosity of $10 \%$ were selected based on the theoretical consideration described in Section 4.4.1. Generation of micrometer-sized pore-spanning membranes required a hydrophilic functionalization of the substrate. For functionalization, a gold layer of approximately 30-40 nm was evaporated onto the substrate and a self-assembled 
monolayer was formed by incubation of the substrate in 6-mercapto-1-hexanol. Spreading of GUVs led to the formation of pore-spanning membranes. Even though iso-osmolar conditions were used, the formed pore-spanning membranes were not entirely planar (Figure 4.15 A). Application of an osmolarity gradient led to an influx of water into the cavities and the membranes bulged from the surface as a function of the applied osmolarity gradient (Figure 4.15 B-D). Membranes located at the patch border were less influenced by the osmolarity gradient compared to membranes in the patch center. This could be observed by the height of the PSMs located at the patch border being lower compared to the heights of the PSMs in the patch center. This effect was not observed when using substrates with smaller pore diameters and lower porosity (Section 4.2).
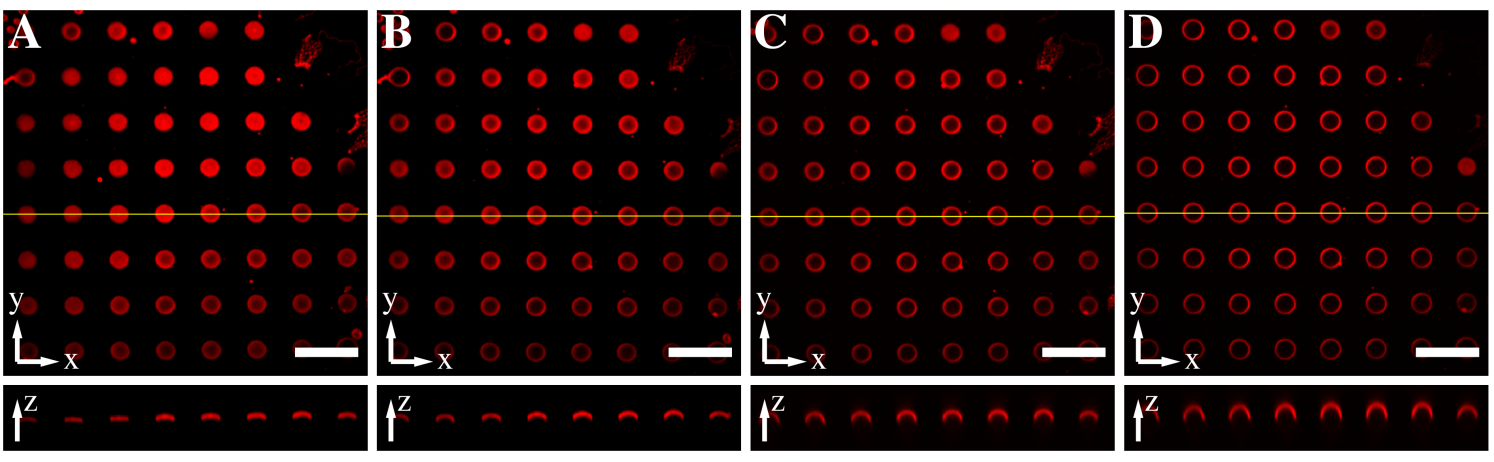

Figure 4.15: SDCLM images of pore-spanning membranes (DPhPC/Texas Red, 99.5:0.5) in PBS $\left(O_{\text {cavity, } 0}=100 \mathrm{mOsmol} / \mathrm{L}\right)$. The yellow line in the $x$ - $y$-plane indicates the position of the $z$-profiles. The bottom images show the geometry of the protruded PSMs after application of different osmolarity gradients. Osmolarity gradients of: A: $0 \mathrm{mOsmol} / \mathrm{L}$. B: $3 \mathrm{mOsmol} / \mathrm{L}$, C: $6 \mathrm{mOsmol} / \mathrm{L}$ and D: $12 \mathrm{mOsmol} / \mathrm{L}$ were applied. Height of PSMs increases as a function of the applied osmolarity gradient. Height of $z$-profiles: $5 \mu \mathrm{m}$, scale bars: $10 \mu \mathrm{m}$.

To increase the accuracy of the height determination, protruded pore-spanning membranes on micrometer-sized pores were imaged by SDCLM. Image acquisition by SDCLM requires shorter exposure times and allows to image $z$-stacks with more planes within a shorter period of time. The diameter of the pinholes of the spinning disc laser microscope is optimized for objectives with $100 \times$ magnification and thus the confocality decreases when using an objective with a lower magnification. The field of view of the $100 \times$ magnification objective was smaller than that of the CLSM which was compensated by a higher porosity of the porous substrates with pore radii of $1.75 \mu \mathrm{m}$ compared to the substrates with pore radii of $0.425 \mu \mathrm{m}$ 
(Section 4.2). Thus, a similar number of $z$-stacks with smaller voxel size compared to CLSM could be imaged. The larger number of slices per $z$-stack allows to obtain intensity profiles with more data points, which increases the accuracy of the intensity profile fit and thereby of the height determination.

Data evaluation was performed with a custom written Matlab script using the same evaluation strategy which was used for the height determination of nanometer-sized protruded pore-spanning membranes (Section 4.2). The pore grid (Figure 4.16 A, yellow) was determined by user input and refined using a watershed algorithm. An intensity profile in $z$-direction was automatically obtained in the pore center (Figure 4.16 A, red). Four intensity profiles were measured centered between the four diagonal neighbors of each pore (Figure $4.16 \mathrm{~A}$, blue). The $z$-position of the membrane at the pore rim was determined by averaging the position obtained by fitting a Gaussian function to the four intensity profiles. This allowed to correct for a possible tilting of the substrate. Subtraction of the maxima position of the intensity profiles yielded the height of the pore-spanning membranes (Figure 4.16 B). Heights of the protruded pore-spanning membranes were measured as a function of the applied osmolarity gradient and are shown in Figure 4.16 B. Osmolarity gradients larger than $15 \mathrm{mOsmol} / \mathrm{L}$ were not evaluated as the geometry of the protruded pore-spanning membranes started to deviate from the assumed spherical cap geometry. The height of the PSMs increased as a function of the applied osmolarity gradient exceeding the pore radius of $1.75 \mu \mathrm{m}$ for applied osmolarity gradients larger than $9 \mathrm{mOsmol} / \mathrm{L}$.

Contrary to experiments on smaller pores (Section 4.2 no planar membranes were formed by spreading of GUVs. A membrane height of $0.5 \pm 0.3 \mu \mathrm{m}$ at iso-osmolar conditions was measured deviating from zero for the expected planar membrane topology. Osmolarity differences between the sucrose solution within the GUVs used for spreading and the surrounding buffer would be a possible explanation for the non-planarity of the membrane. Attempts to readjust the osmolarities of the different buffers used, to change the method of GUV addition and the GUV incubation time before rinsing were not successful to produce planar PSMs. To compensate for the non-planarity at iso-osmolar conditions the osmolarity inside the cavity $\left(O_{\text {cavity, } 0}\right)$ was added as a second fitting parameter (equation 4.7$)$. Fitting yielded $O_{\text {cavity }, 0}=104 \pm 2 \mathrm{mOsmol} / \mathrm{L}$ and a membrane tension of $2 \pm 7 \mathrm{mN} / \mathrm{m}$. 

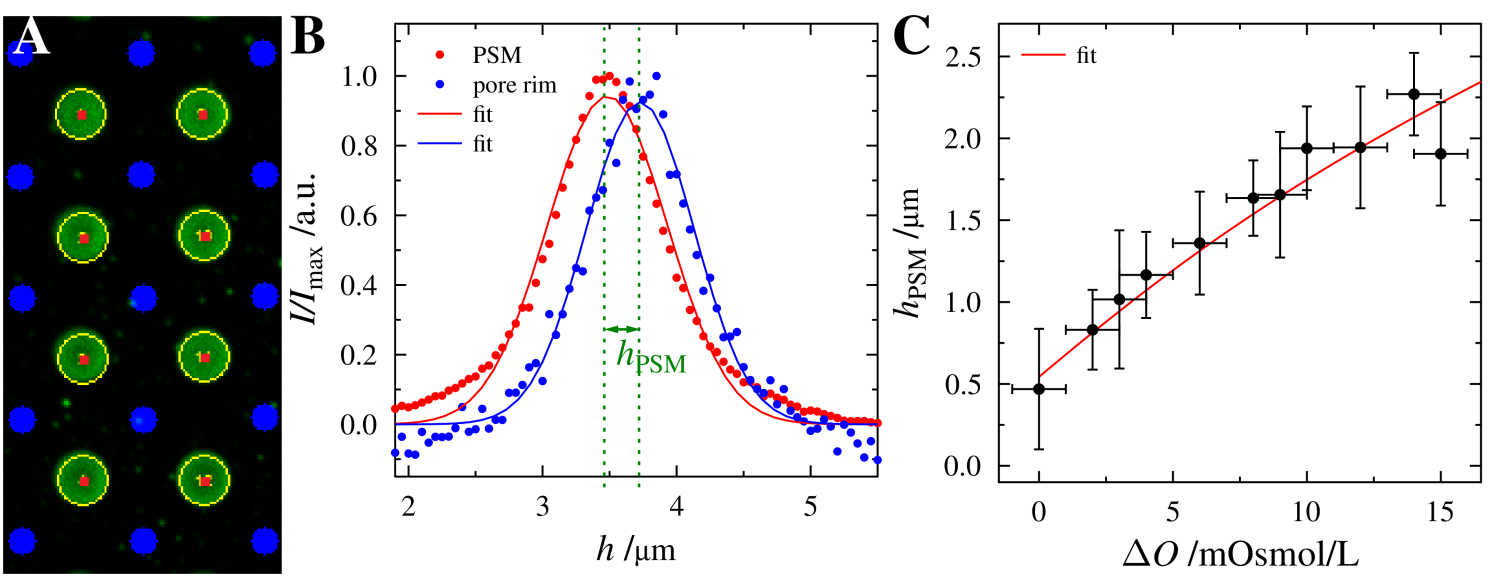

Figure 4.16: A: Overlay of a SDCLM image of pore-spanning membranes (DPhPC/Atto $488 \mathrm{DPPE}, 99: 1$, green) on a porous substrate with a pore radius of $1.75 \mu \mathrm{m}$, the pore grid detected by the evaluation script (yellow) and the regions for measuring the intensity profiles (blue, red). B: Representative normalized fluorescence intensity profiles of a protruded pore-spanning membrane and the membrane located on the pore rim. A Gaussian function was fitted to the intensity of both membranes to determine the height of the protruded PSM $h_{\mathrm{PSM}}$. C: Heights of pore-spanning membranes (DPhPC/Atto 488 DPPE, 99:1) as a function of the applied osmolarity gradient $\left(O_{\text {cavity }, 0}=100 \mathrm{mOsmol} / \mathrm{L}\right)$. Errors of the membrane height are the standard deviation of the Gaussian distribution and errors of the applied osmolarity gradient are the uncertainty of the osmometric measurements. Fitting equation 4.7 to the data (red line) yields: $\sigma=2 \pm 7 \mathrm{mN} / \mathrm{m}$ and $O_{\text {cavity }, 0}=104 \pm 2 \mathrm{mOsmol} / \mathrm{L}$.

Equation 4.7 was then fitted to the height of each protruded pore-spanning membrane as a function of the applied osmolarity gradient to determine the lateral membrane tension of the individual PSMs yielding a distribution of the membrane tensions (Figure 4.17). The obtained distribution of the lateral membrane tension of pore-spanning membranes on a hydrophilically functionalized porous substrate was much broader than measured for PSMs on substrates with open pores by means of atomic force microscopy. 119, 139] Fitting the data also yielded a relevant fraction of membranes with negative membrane tensions. This is physically not meaningful and attributed to uncertainties in fitting the data and the non-planarity of the PSMs at iso-osmolar conditions.

Even though using optimized experimental conditions for maximum sensitivity and robustness to uncertainties, the determination of the lateral membrane tension of pore-spanning membranes by correlating their height with the applied osmolarity gradient yielded in a too broad distribution of membrane tensions including a relevant fraction of membranes with physically not meaningful results. Thus, 
determining the lateral tension by fitting equation 4.7 to the height of each PSM as a function of the applied osmolarity gradient was more inaccurate than by fitting equation 4.7 to the mean membrane height as a function of the applied osmolarity gradient. Therefore, atomic force microscopy experiments were conducted as they have been shown of being capable to measure the lateral membrane tension of pore-spanning membranes with high accuracy.

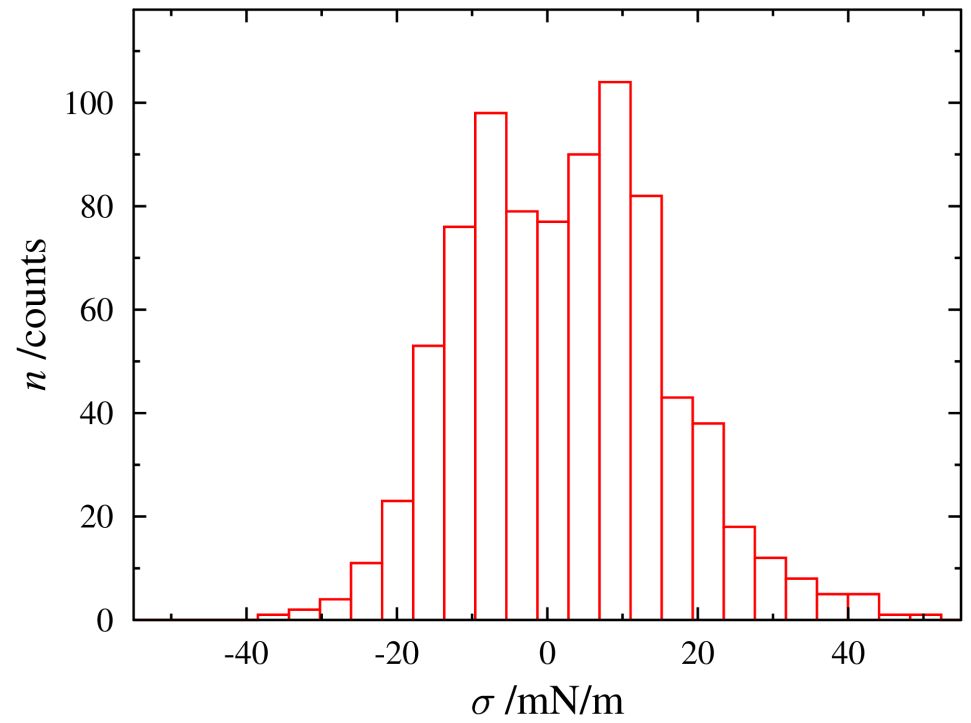

Figure 4.17: Histogram of lateral membrane tensions of PSMs obtained by fitting equation 4.7 to the heights of the pore-spanning membranes as a function of the applied osmolarity gradient.

\subsubsection{Lateral membrane tension of micrometer-sized protruded pore-spanning membranes measured by atomic force microscopy}

The distribution of the tension determined for micrometer-sized pore-spanning membranes was too large for calculating membrane tensions with sufficient accuracy using the fluorescence based approach applying the Young-Laplace theory. To directly measure the lateral tension of protruded pore-spanning membranes atomic force microscopy experiments were conducted. They allow to probe the response of protruded pore-spanning membranes by indentation with a cantilever in order to determine the membrane tension with high accuracy. For fluorescence microscopy 
experiments porous silicon substrates were used and the PSMs were imaged using an upright microscope. Spreading of GUVs led to the formation of membrane patches which were localized by fluorescence microscopy for the AFM experiments. Localization using an upright microscope was not feasible in combination with atomic force microscopy measurements and therefore an inverse microscope had to be used requiring transparent substrates. Pore-spanning membranes were formed on porous glass substrates with a porosity of $20 \%$, pore radii of $1.75 \mu \mathrm{m}$ and a pore height of $8 \mu \mathrm{m}$. Unfortunately, a fraction of the pores was interconnected as an artifact of the manufacturing process (Figure 4.18). Interconnected pores were not sealed after formation of a pore-spanning membrane as water could be exchanged with the bulk solution via the connection. This led to a breakdown of the osmolarity gradient between pore interior and bulk solution. Therefore, membranes formed on interconnected pores were not protruded by the applied osmolarity gradient and remained planar. As a result of the interconnection protruded and planar pore-spanning membranes were probed after application of an osmolarity gradient on the same substrate.

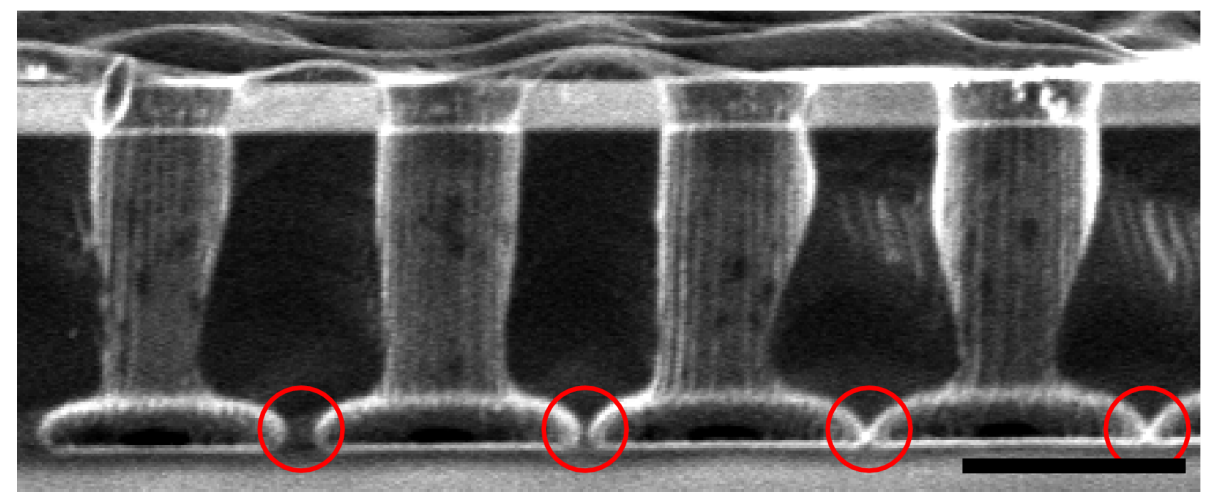

Figure 4.18: Representative scanning electron microscopy image of the cross section of a porous $\mathrm{SiO}_{2}$ substrate with pore radii of $1.75 \mu \mathrm{m}$, a pore depth of $8 \mu \mathrm{m}$ and a porosity of $20 \%$. The pores are partially interconnected at the pore bottom (red circles). Scale bar: $5 \mu \mathrm{m}$.

A typical force volume image of pore-spanning membranes measured on porous $\mathrm{SiO}_{2}$ substrates after application of an osmolarity gradient of $20 \mathrm{mOsmol} / \mathrm{L}$ is shown in Figure 4.19 A. Each pixel represents a force-distance curve of the cantilever approaching the sample and indenting it until a previously defined force is reached. The color code indicates the absolute height when the predefined force 
was reached and the indentation stopped. Representative force-distance curves measured on non-covered pores, membrane-covered open pores and membranecovered closed pores are shown in Figure 4.19B.

The assignment of a pore being closed or open was achieved by measuring the membrane height. The height of the pore-spanning membrane was calculated by subtracting the absolute height of the contact point of the cantilever with the pore rim (Figure 4.19 B, black) from the contact point of the cantilever with the porespanning membrane (Figure 4.19 B, blue, green, red). Non-covered pores adjacent to protruded membranes led to a decrease of the membrane height of the protruded PSMs. If the membrane height was larger than $100 \mathrm{~nm}$ after applying an osmolarity gradient, a pore was assumed to be closed since the membrane was protruded by the applied osmolarity gradient (Figure 4.19A, blue). Membranes with a height of $-100 \mathrm{~nm}$ to $100 \mathrm{~nm}$ were considered to span an open pore (Figure 4.19A, green). For non-covered pores apparent heights smaller than $-100 \mathrm{~nm}$ were measured (Figure 4.19A, red).
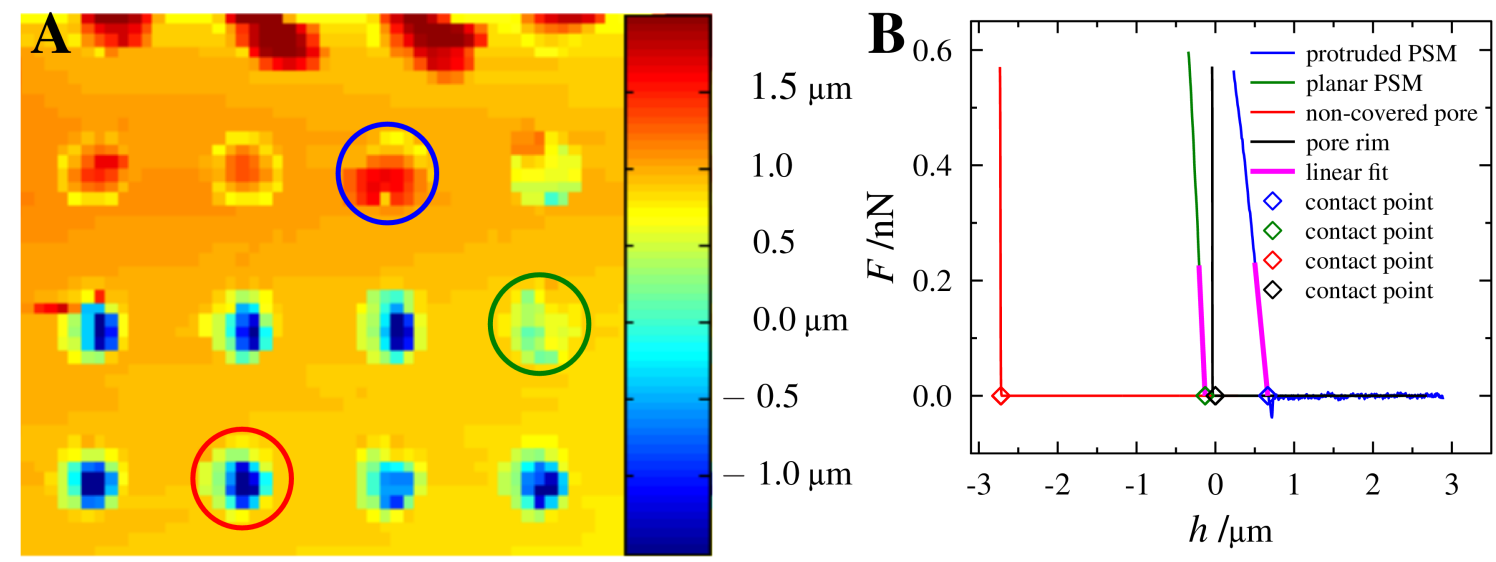

Figure 4.19: A: Force volume image of pore-spanning membranes (DPhPC/Texas Red, 99:1) after application of an osmolarity gradient of $20 \mathrm{mOsmol} / \mathrm{L}$. Protruded (blue circle) and planar (green circle) pore-spanning membranes were imaged together with non-covered pores (red circle). Pore diameter: $3.5 \mu \mathrm{m}$. B: Representative force-distance curves measured in the center of the regions marked in $\mathbf{A}$ and on the pore rim (black curve). The contact points are marked with diamonds.

Fluorescence microscopy of protruded pore-spanning membranes revealed membranes located at the border of a membrane patch having smaller heights compared to membranes in the patch center (Figure 4.15). Using atomic force microscopy, it could be shown that the height of membranes neighbored to an open pore was 
reduced similar to membranes located at the border of the membrane patch as observed by fluorescence microscopy. Due to the large number of open pores a correlation of the height of the protruded pore-spanning membranes measured by atomic force microscopy to the applied osmolarity gradient was not possible.

The overall mechanical response to indentation is given by the slope of the forcedistance curves. It is mainly determined by pre-stress of the membrane caused by adhesion to the substrate. [119, 141] The lateral membrane tension was calculated for the experimentally used parameters such as the radius of the cantilever tip $\left(r_{\text {tip }}=25 \mathrm{~nm}\right)$, the pore radius $\left(r_{\text {pore }}=1.75 \mu \mathrm{m}\right)$ and the apparent spring constant. Latter was obtained by linear fitting of the part of the force-distance curves where the cantilever indented the membranes (Figure 4.19 B, magenta). ${ }^{120}$ Lateral membrane tensions of $2 \pm 1 \mathrm{mN} / \mathrm{m}(n=171)$ measured for protruded pore-spanning membranes on closed pores and $2 \pm 1 \mathrm{mN} / \mathrm{m}(n=94)$ measured on open pores were determined. Comparison of the membrane tensions measured with atomic force microscopy with those determined by fitting the height of protruded porespanning membranes measured with confocal fluorescence microscopy shows that all membrane tensions are within the expected range of $1-3 \mathrm{mN} / \mathrm{m}$ measured for PSMs of different lipid compositions on hydrophilically functionalized porous substrates with open pores. 119, 139]

The lateral tension of lipid bilayers was successfully measured by optical microscopy using protruded pore-spanning membranes on porous substrates with pore radii of $425 \mathrm{~nm}$. For larger pores with radii of $1.75 \mu \mathrm{m}$ the distribution of the membrane tensions was too broad for an accurate determination of the membrane's lateral tension. It could be shown that the model system of protruded porespanning membranes can be used to analyze membrane-protein interactions involving changes in the lateral membrane tension. Binding of ENTH to protruded porespanning membranes leads to growth and deflation of pore-spanning membranes. Uncertainties of the osmolarity measurements and the large influence of the pore geometry resulted in a high deviation of the individual measurements limiting the application. To overcome these experimental limitations and to perform experiments with lipid bilayers having a lower membrane tension, experiments with giant unilamellar vesicles were conducted. 


\subsection{Adhered GUVs as a model system to analyze tension dependent processes}

During the last years giant unilamellar vesicles (GUVs) have been become an important model system for cell membranes. They are relatively easy to produce by electro-formation or swelling in buffer. ${ }^{95}$ Electro-formation results in a more homogeneous distribution of vesicles compared to swelling. ${ }^{[126]}$ Membrane-protein interactions and phase separation of lipid bilayers have been studied extensively using GUVs. [55, 153, 154] Micropipette aspiration, tether pulling or compression of vesicles are often used to determine the mechanical properties of GUVs and involve area dilatation to measure and tune the vesicles' membrane tension. $155-157$ In this thesis, biotinylated GUVs were immobilized on an avidin-coated glass substrate. Using sucrose buffers containing different concentrations of $\mathrm{Mg}^{2+}$, the adhesion strength of the vesicles to the substrate was tuned. The lateral membrane tension was then calculated from vesicle geometry which allows to study binding of ENTH as a function of the lateral membrane tension.

\subsubsection{Theoretical model}

Contact of vesicles to an adhesive substrate causes attractive forces between the vesicle's membrane and substrate. The free energy $F$ in the strong adhesion limit is mainly determined by elastic stretching of the membrane and its adhesion to the substrate as shown in equation 4.9 .

$$
F=\frac{1}{2} K_{A} \frac{\Delta A^{2}}{A_{\mathrm{v}}}-A_{\mathrm{i}} \gamma_{\mathrm{ad}}
$$

The contribution from elastic stretching depends on the area compressibility modulus $K_{A}$ and the increase in area $\Delta A=A_{\mathrm{ad}}-A_{\mathrm{v}}$ of a vesicle with an area $A_{\mathrm{v}}$ to an adhered vesicle with the area $A_{\mathrm{ad}}$. A circular Area $A_{\mathrm{i}}$ with the radius $r_{\mathrm{i}}$ and the adhesion energy per unit area $\gamma_{\mathrm{ad}}$ determines the energy contribution from adhesion. The adhesion of a vesicle stops when the reduction in free energy caused by the adhesion of the membrane to the substrate equals the increase in free energy caused by the increase in membrane tension as a result of the area dilatation. 158, 159 
The membrane tension of a vesicle before adhesion has been described theoretically. $160-162$ Thermal excitation gives rise to membrane fluctuations with different wavelengths. The area of a fluctuating membrane is larger than that of its projection. The fluctuating membrane is compressed compared to a planar membrane and the tension of the membrane $\sigma$ is renormalized by thermal fluctuations. The observable tension of a fluctuating membrane $\sigma$ is given by:

$$
\sigma=\Delta \sigma-\frac{K_{A} k_{\mathrm{B}} T}{8 \pi \kappa} \ln \left(\frac{\frac{\sigma A_{\mathrm{v}}}{\pi^{2} \mathcal{K}}+1}{\frac{\sigma a_{1}}{\pi^{2} \mathcal{K}}+1}\right),
$$

with $k_{\mathrm{B}}$ being the Boltzmann constant, $T$ the absolute temperature, $a_{1}$ the area of a lipid, $\kappa$ the bending rigidity and $\Delta \sigma$ the amount which the membrane must be stretched to result in an observable tension. [163] It was derived by integration of all fluctuations with $q_{\min }=\pi / A_{\mathrm{v}}$ and $q_{\max }=\pi / a_{1}$ being the lower and upper vector of the membrane fluctuations. For low membrane tensions $\left(\sigma \ll \pi^{2} \kappa / a_{1}\right)$ the denominator is close to unity and equation 4.10 simplifies to:

$$
\sigma=\Delta \sigma-\frac{K_{A} k_{\mathrm{B}} T}{8 \pi \kappa} \ln \left(\frac{\sigma A_{\mathrm{v}}}{\pi^{2} \kappa}+1\right) .
$$

For relevant membrane tensions $\left(K_{A} \gg \sigma \gg \pi^{2} \kappa / A_{\mathrm{v}}\right)$ is $\left(\sigma A_{\mathrm{v}}\right) /\left(\pi^{2} \kappa\right) \gg 1$ and equation 4.11 simplifies to:

$$
\sigma=\Delta \sigma-\frac{K_{A} k_{\mathrm{B}} T}{8 \pi \kappa} \ln \left(\frac{\sigma A_{\mathrm{v}}}{\pi^{2} \kappa}\right)
$$

The pre-tension of a free vesicle $\left(\sigma_{0}\right)$ is caused by its finite vesicle size and the resulting cut-off of long wavelength undulations. The increase in membrane tension of a tension-free planar membrane is proportional to fractional area expansion $(\triangle A / A)$ with $K_{A}$ being the proportionality factor (equation 4.13).

$$
\Delta \sigma=K_{A} \frac{\Delta A}{A}
$$

Combination of equation 4.13 and 4.12 allows to calculate the membrane tension of an adhered vesicle as follows:

$$
\sigma=K_{A} \frac{\Delta A}{A_{\mathrm{v}}}-\frac{K_{A} k_{\mathrm{B}} T}{8 \pi \kappa} \ln \left(\frac{\sigma A_{\mathrm{v}}}{\pi^{2} \kappa}\right) .
$$


The correlation between fractional area increase and lateral membrane tension was measured by Evans and Rawicz using micropipette aspiration. [164] They quantitatively confirmed the theoretically predicted renormalization of the membrane tension and proved the flattening of fluctuations at high membrane tensions. It is important to note that Evans and Rawicz defined the area of a tension-free fluctuating vesicle as reference state, whereas Helfrich and Servus used the area projection of a tension-free fluctuating vesicle. To compare their experimental results with the theoretically predicted ones, the contribution of the different reference states must be considered. Therefore, the membrane tension of the free vesicle is subtracted from the tension of the adhered vesicle (equation 4.14 ) which leads to:

$$
\frac{\Delta A}{A_{\mathrm{v}}}=\frac{\sigma-\sigma_{0}}{K_{A}}+\frac{k_{\mathrm{B}} T}{8 \pi \kappa} \ln \left(\frac{\sigma}{\sigma_{0}}\right)
$$

For small membrane tensions $(\sigma<0.5 \mathrm{mN} / \mathrm{m})$ undulations are suppressed when the area of a vesicle is expanded. At higher tensions undulations are reduced in amplitude and the increase of the fractional area results from the direct stretching of lipids. These calculations are only valid for iso-osmolar conditions since the undulations and so the vesicles' excess membrane are a function of the osmolarity gradient between GUV interior and exterior.

The theory can be applied to calculate the membrane tension of a GUV depending on its geometry. GUVs adhering on solid substrates change their shape from a sphere to a spherical cap (Figure 4.20).
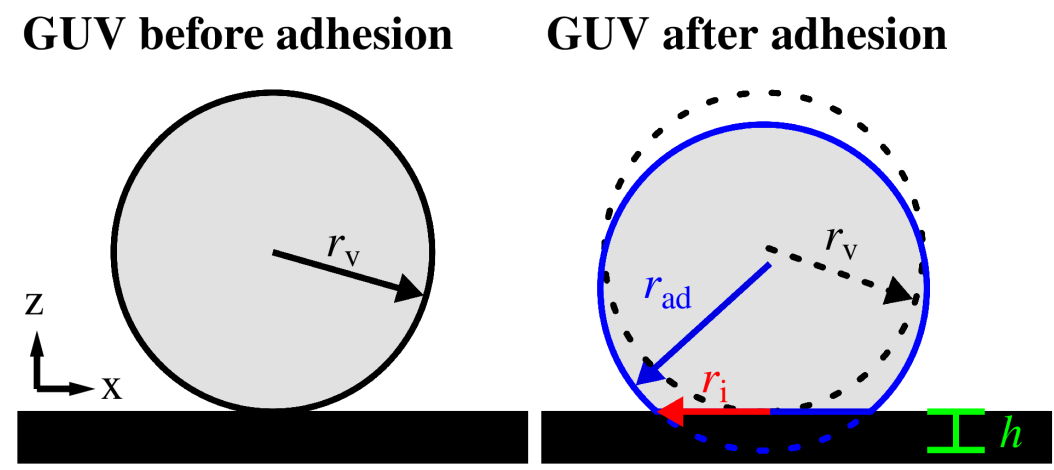

Figure 4.20: Scheme of a GUV on a surface before and after adhesion. Without adhesion, the GUV geometry can be approximated as a sphere (radius $r_{\mathrm{v}}$ ). After adhesion it forms a spherical cap with the radius of $r_{\text {ad }}$ and a circular contact area (with the radius of $r_{\mathrm{i}}$ ). 
During the adhesion process the vesicle volume remains constant due to the low permeability of biological membranes to water [165, 166]. Adhesion and subsequent geometry change leads to an increase of the GUV surface area translating to an increase of the lateral membrane tension. Equation 4.16 accounts for the volume conservation of a sphere with a radius $r_{\mathrm{v}}$ forming a spherical cap with a radius $r_{\mathrm{ad}}$ and a contact area with the radius $r_{i}$.

$$
\frac{4}{3} \pi r_{\mathrm{v}}^{3}=\frac{4}{3} \pi r_{\mathrm{ad}}^{3}-\frac{\pi h}{6}\left(3 r_{\mathrm{i}}^{2}+h^{2}\right)
$$

Experimentally the three dimensional structure of immobilized GUVs compared to moving, non-adhered vesicles is easier to image. The radius of a GUV before adhesion can then be calculated as a function of the GUV radius after adhesion by rearranging equation 4.16 .

$$
r_{\mathrm{v}}=\sqrt[3]{r_{\mathrm{ad}}^{3}-\frac{h}{8}\left(3 r_{\mathrm{i}}^{2}+h^{2}\right)}
$$

The height of the spherical cap $h$ (Figure 4.20) is calculated as a function of $r_{\text {ad }}$ and $r_{\mathrm{i}}$ as shown below.

$$
h=r_{\mathrm{ad}}-\sqrt{r_{\mathrm{ad}}^{2}-r_{\mathrm{i}}^{2}}
$$

Using the geometrical constrains it is possible to calculate the fractional area of a spherical cap as a function of the adhesion strength using equation 4.19 .

$$
\frac{\Delta A}{A}=\frac{A_{\mathrm{ad}}-A_{\mathrm{v}}}{A_{\mathrm{v}}}=\frac{4 \pi r_{\mathrm{ad}}^{2}-2 \pi r_{\mathrm{ad}} h+\pi r_{\mathrm{i}}^{2}-4 \pi r_{\mathrm{v}}^{2}}{4 \pi r_{\mathrm{v}}^{2}}
$$

The fractional area of a spherical vesicle with a radius of $10 \mu \mathrm{m}$ before adhesion is plotted as a function of the contact area radius after adhesion (Figure 4.21 A). The radius of an adhered vesicle is not constant and increases as a function of $r_{\mathrm{i}}$ (Figure $4.21 \mathrm{~B}$ ). 

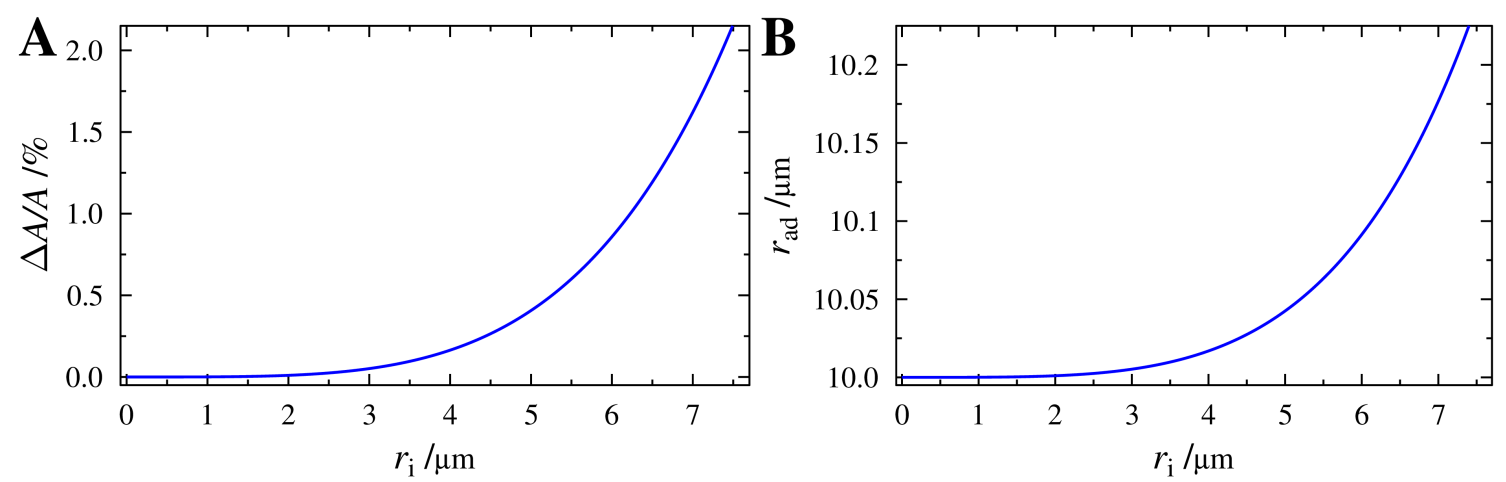

Figure 4.21: Fractional area $(\mathbf{A})$ and radius of the adhered vesicle $(\mathbf{B})$ with a radius of $10 \mu \mathrm{m}$ before adhesion as a function of the contact radius.

To obtain a dimensionless quantity the contact area radius $\left(r_{\mathrm{i}}\right)$ is normalized to the radius of the adhered vesicle $\left(r_{\mathrm{ad}}\right)$. The fractional area of the spherical cap is then calculated by combination of equation 4.19 and 4.18 where $r_{\mathrm{i}}, r_{\mathrm{v}}$ and $r_{\mathrm{ad}}$ are substituted with the variables normalized to $r_{\text {ad }}$ (equation 4.20):

$$
\frac{\Delta A}{A}=\frac{4 \pi-2 \pi\left(1-\sqrt{1-\left(\frac{r_{\mathrm{i}}}{r_{\mathrm{ad}}}\right)^{2}}\right)+\pi\left(\frac{r_{\mathrm{i}}}{r_{\mathrm{ad}}}\right)^{2}-4 \pi\left(\frac{r_{\mathrm{v}}}{r_{\mathrm{ad}}}\right)^{2}}{4 \pi\left(\frac{r_{\mathrm{v}}}{r_{\mathrm{ad}}}\right)^{2}} .
$$

By combining equation 4.17 and 4.18 and by substitution of $r_{\mathrm{i}}, r_{\mathrm{v}}$ and $r_{\mathrm{ad}}$ with the variables normalized to $r_{\mathrm{ad}}$ the normalized radius of a free vesicle as a function of $r_{\mathrm{i}}$ and $r_{\mathrm{ad}}$ can be calculated as shown below:

$$
\frac{r_{\mathrm{v}}}{r_{\mathrm{ad}}}=\sqrt[3]{1-\frac{2-\left(\frac{r_{\mathrm{i}}}{r_{\mathrm{ad}}}\right)^{2} \sqrt{1-\left(\frac{r_{\mathrm{i}}}{r_{\mathrm{ad}}}\right)^{2}}-2 \sqrt{1-\left(\frac{r_{\mathrm{i}}}{r_{\mathrm{ad}}}\right)^{2}}}{4}} .
$$

The fractional area can then be calculated by combination of equation 4.20 and 4.21 (equation 4.22):

$$
\frac{\Delta A}{A}=\frac{4 \pi-2 \pi\left(1-\sqrt{1-\left(\frac{r_{\mathrm{i}}}{r_{\mathrm{ad}}}\right)^{2}}\right)+\pi\left(\frac{r_{\mathrm{i}}}{r_{\mathrm{ad}}}\right)^{2}}{4 \pi\left(\sqrt[3]{1-\frac{2-\left(\frac{r_{\mathrm{i}}}{r_{\mathrm{ad}}}\right)^{2} \sqrt{1-\left(\frac{r_{\mathrm{i}}}{r_{\mathrm{ad}}}\right)^{2}}-2 \sqrt{1-\left(\frac{r_{\mathrm{i}}}{r_{\mathrm{ad}}}\right)^{2}}}{4}}\right)^{2}}-1 .
$$


To calculate the lateral membrane tension of adhered vesicles as a function of $r_{\mathrm{i}} / r_{\mathrm{ad}}$ for different pre-tensions the combination of equation 4.15 and 4.22 was solved numerically (equation 4.23).

$$
\frac{\sigma-\sigma_{0}}{K_{A}}+\frac{k_{\mathrm{B}} T}{8 \pi \kappa} \ln \left(\frac{\sigma}{\sigma_{0}}\right)=\frac{4 \pi-2 \pi\left(1-\sqrt{1-\left(\frac{r_{\mathrm{i}}}{r_{\mathrm{ad}}}\right)^{2}}\right)+\pi\left(\frac{r_{\mathrm{i}}}{r_{\mathrm{ad}}}\right)^{2}}{4 \pi\left(\sqrt[3]{1-\frac{2-\left(\frac{r_{\mathrm{i}}}{r_{\mathrm{ad}}}\right)^{2} \sqrt{1-\left(\frac{r_{\mathrm{i}}}{r_{\mathrm{ad}}}\right)^{2}}-2 \sqrt{1-\left(\frac{r_{\mathrm{i}}}{r_{\mathrm{ad}}}\right)^{2}}}{4}}\right)^{2}}-1 .
$$

GUVs weakly adhering to a solid support have a pre-tension ranging from $10^{-3}$ to $10^{-1} \mathrm{mN} / \mathrm{m}$. 167] The area compressibility modulus of GUVs was measured by micropipette aspiration and ranges from 50 to $3300 \mathrm{mN} / \mathrm{m}$ depending on lipid composition and temperature. ${ }^{64,168]}$ Figure 4.22 shows the lateral tension of a GUV composed of DOPC/DOPE (70:30) adhering to a solid support as a function of $r_{\mathrm{i}} / r_{\mathrm{ad}}$ calculated for an area compressibility modulus of $265 \mathrm{mN} / \mathrm{m}$, a bending rigidity of $21 k_{\mathrm{B}} T$ and pre-tensions of $10^{-3} \mathrm{mN} / \mathrm{m}, 10^{-2} \mathrm{mN} / \mathrm{m}$ and $10^{-1} \mathrm{mN} / \mathrm{m} .[167,169]$

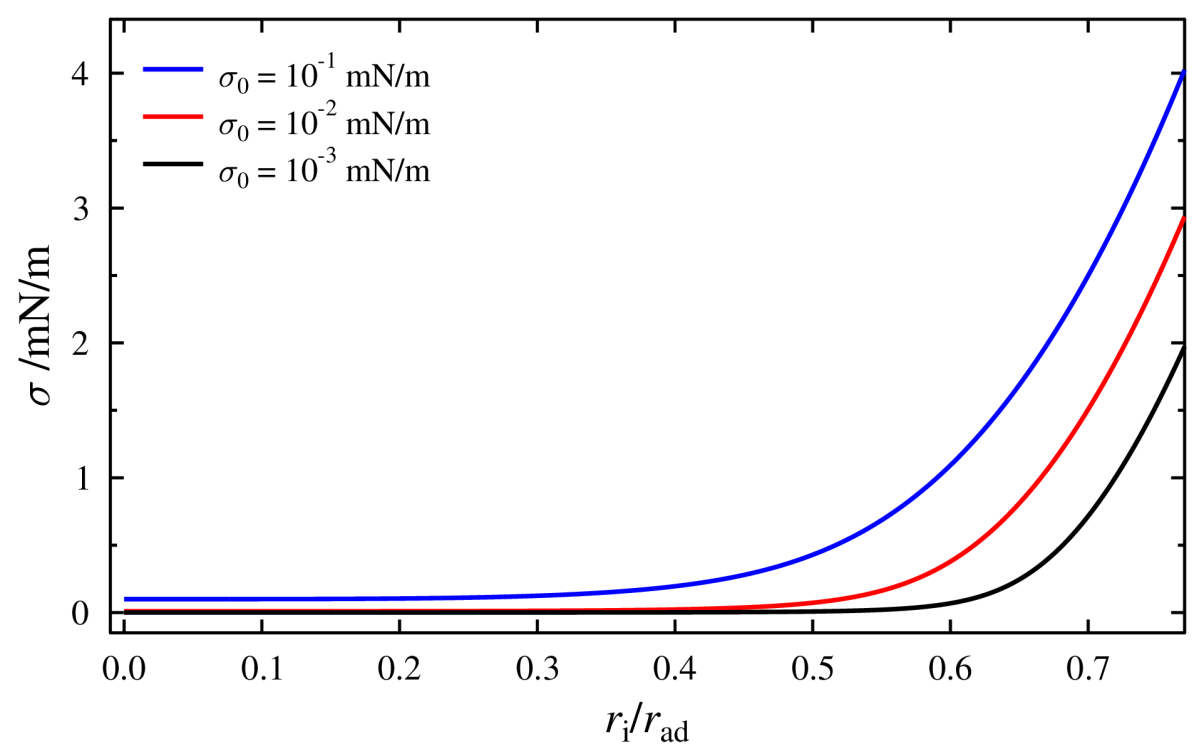

Figure 4.22: Resulting membrane tension of GUVs adhering to a substrate as a function of $r_{\mathrm{i}} / r_{\mathrm{ad}}$ calculated for different pre-tensions. 
For all pre-tensions the lateral membrane tension increases very slowly as long as the excess area, which is stored in undulations, is used to compensate the increase in surface area resulting from the area dilatation during the adhesion process. If the area dilatation exceeds the excess area stored in the undulations, the lateral membrane tension increases rapidly. The ratio of $r_{\mathrm{i}} / r_{\mathrm{ad}}$ for a given lateral tension is smaller for higher pre-tensions since less excess area can be stored in the undulations. Additionally, the pre-tension governs the minimal tension of the vesicle. Adhesion of GUVs results in membrane tensions similar to those measured in cells which allows to analyze biological processes as a function of the lateral membrane tension. 29, 33,-35]

\subsubsection{Adhesion of biotinylated GUVs adhering to an avidin-coated glass surface as a function of the $\mathrm{Mg}^{2+}$ concentration}

GUVs adhering to a solid support have been used to analyze membrane-substrate distances, ${ }^{[170]}$ membrane fluctuations, ${ }^{167]}$ and membrane phase separation. [171] Also adhesion is often used to immobilize vesicles or to analyze membrane-protein interactions [172]. In first experiments $\mathrm{MgF}_{2}$ was deposited onto a glass slide with subsequent vesicle adhesion. The low solubility of $\mathrm{MgF}_{2}$ results in a small concentration of dissolved $\mathrm{Mg}^{2+}$. Addition of calcium ions increased the adhesion of vesicles to the surface. 170 Another possibility which is often used to immobilize vesicles on a surface is to generate attractive forces by the formation of biotin(strept)avidin bonds. [173] In this thesis both strategies were combined to adhere vesicles to a solid support but also to tune the adhesion strength and therewith the lateral tension of the vesicles.

GUVs without (DOPC/DOPE/cap-biotin-DOPE/Atto488-DPPE, 67:30:2:1) and with $\mathrm{PIP}_{2}$ (DOPC/DOPE/cap-biotin-DOPE/Atto488-DPPE/PIP 2 , 66.2:30:2:1:0.8) in sucrose buffer doped with different amounts $(0.5-6 \mathrm{~mm})$ of $\mathrm{Mg}^{2+}$ were added to an avidin-coated glass surface. Biotin-avidin bonds were formed after subsidence of vesicles and their adhesion to the surface. The geometry of the vesicles was imaged by SDCLM and quantified as described in Section 3.8.1. The GUV contact area (Figure 4.23 , red) and the ratio of contact area to the equatorial plane are (Figure 4.23 , blue) increased as a function of the $\mathrm{Mg}^{2+}$ concentration. 

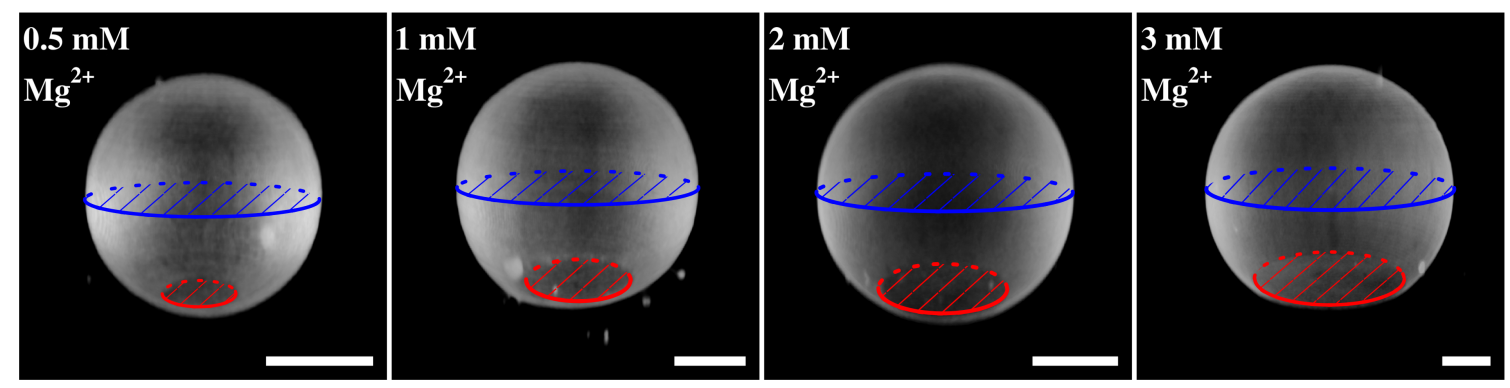

Figure 4.23: Representative 3D reconstructions of SDCLM $z$-stacks of GUVs (DOPC/DOPE/cap-biotin-DOPE/Atto488-DPPE/PIP $2,66.2: 30: 2: 1: 0.8$ ) adhering to an avidin-coated glass surface. The ratio of contact area (red) and equatorial plane area (blue) increases as a function of the $\mathrm{Mg}^{2+}$ concentration. Scale bars: $10 \mu \mathrm{m}$.

To compare the adhesion strength of vesicles with different sizes the ratio of contact radius and GUV radius before adhesion $\left(r_{\mathrm{i}} / r_{\mathrm{ad}}\right.$, Section 4.5.1) was calculated. For each $\mathrm{Mg}^{2+}$ concentration 31 to 101 vesicles with an average diameter of $18 \pm 5 \mu \mathrm{m}$ were imaged $(n=413)$. The mean ratio of $r_{\mathrm{i}} / r_{\mathrm{ad}}$ increased from $0.19 \pm 0.04$ $(n=31)$ at $1 \mathrm{mM} \mathrm{Mg}^{2+}$ to $0.53 \pm 0.09(n=70)$ at $6 \mathrm{mM} \mathrm{Mg}^{2+}$ for GUVs lacking $\mathrm{PIP}_{2}$ (Figure $4.24 \mathrm{~A}$ ).

Vesicles containing the receptor lipid $\mathrm{PIP}_{2}$ showed qualitatively the same reaction to the rising $\mathrm{Mg}^{2+}$ concentration as their $\mathrm{PIP}_{2}$-free counterpart. However, larger ratios of $r_{\mathrm{i}} / r_{\text {ad }}$ were measured compared to $\mathrm{PIP}_{2}$-free GUVs at identical $\mathrm{Mg}^{2+}$ concentrations. The adhesion strength of the $\mathrm{PIP}_{2}$-containing vesicles was initially higher and therefore lower concentrations of $\mathrm{Mg}^{2+}$ were used. The weakest adhesion of $\mathrm{PIP}_{2}$-containing vesicles was measured in the presence of $0.5 \mathrm{~mm}$ $\mathrm{Mg}^{2+}$. Lower concentrations of $\mathrm{Mg}^{2+}$ could not be used since the vesicles did not reach the substrate surface due to the absence of a density gradient between GUV interior and exterior. The density gradient was a result of the $\mathrm{MgCl}_{2}$ content in the sucrose buffer. As $\mathrm{MgCl}_{2}$ dissociates into three ions, the sucrose concentration had to be lowered by three times the $\mathrm{MgCl}_{2}$ concentration to maintain isoosmolarity. To prove the absence of any osmolarity gradient GUVs were measured after $2 \mathrm{~h}$ and their geometry was compared to those measured directly after subsidence and adhesion. No significant differences of $r_{\mathrm{i}} / r_{\mathrm{ad}}$ were measured regardless of the incubation time before imaging. For each $\mathrm{Mg}^{2+}$ concentration 79 to 96 GUVs with an average diameter of $18 \pm 5 \mu \mathrm{m}$ were analyzed $(n=445)$. The ratio of $r_{\mathrm{i}} / r_{\mathrm{ad}}$ increased from $0.46 \pm 0.07(n=84)$ at $0.5 \mathrm{mM} \mathrm{Mg}^{2+}$ to $0.63 \pm 0.06(n=96)$ at $3 \mathrm{~mm}$ 
$\mathrm{Mg}^{2+}$ (Figure 4.24 B). $\mathrm{PIP}_{2}$-containing vesicles also had a narrower distribution of $r_{\mathrm{i}} / r_{\mathrm{ad}}$ which was attributed to electrostatic interactions of $\mathrm{PIP}_{2}$ with $\mathrm{Mg}^{2+}$.
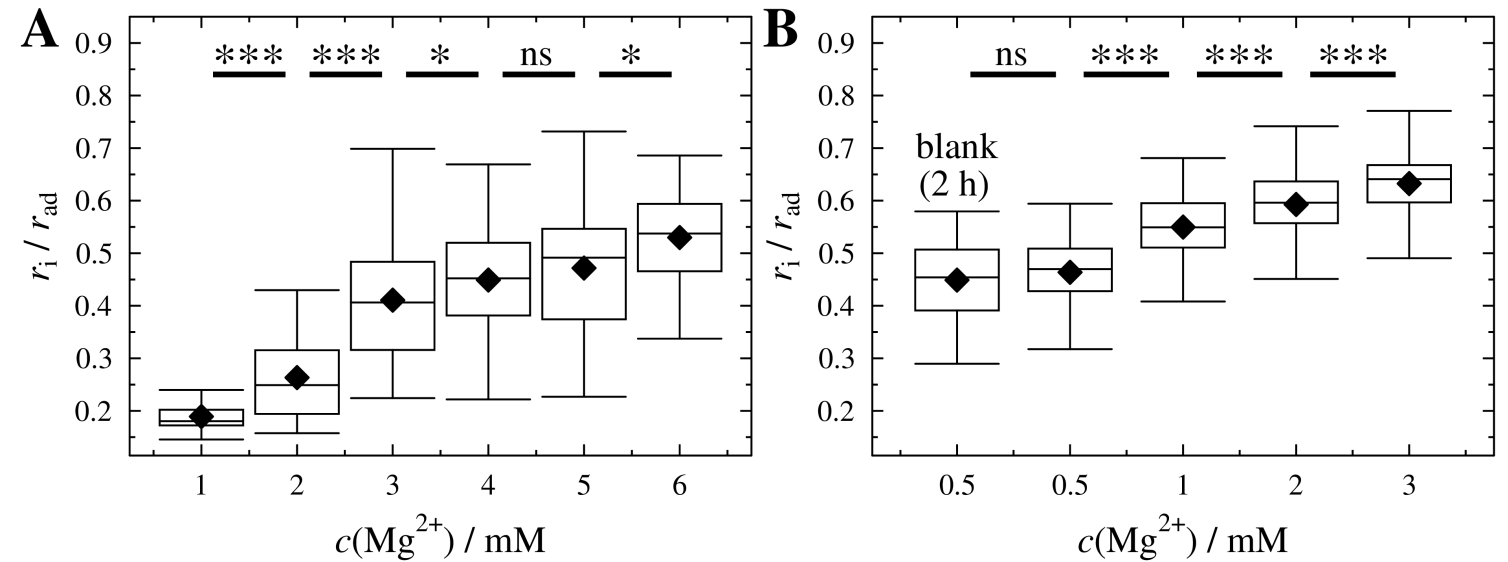

Figure 4.24: Ratio of contact area radius to radius of the adhered vesicle $\left(r_{\mathrm{i}} / r_{\mathrm{ad}}\right)$ at different $\mathrm{Mg}^{2+}$ concentrations. Lower ratios of $r_{\mathrm{i}} / r_{\mathrm{ad}}$ were measured for GUVs (DOPC/DOPE/capbiotin-DOPE/Atto488-DPPE, 67:30:2:1) lacking the receptor lipid $\mathrm{PIP}_{2}$ (A) compared to those containing $0.8 \% \mathrm{PIP}_{2}$ (B, DOPC/DOPE/cap-biotin-DOPE/Atto488-DPPE/PIP 2 , 66.2:30:2:1:0.8). Statistical Mann-Whitney $U$ test: ${ }^{* * *}: p<0.001,{ }^{*}: p<0.05$, ns: not significant.

\subsubsection{Adhesion energy and lateral membrane tension of GUVs adhering to an avidin-coated glass surface}

The lateral tension of vesicles adhering to an avidin-coated glass surface was calculated from the measured ratios of $r_{\mathrm{i}} / r_{\mathrm{ad}}$ as a function of the $\mathrm{Mg}^{2+}$ concentrations by numerically solving equation 4.23 (Figure 4.25). The determined lateral membrane tension depends on the pre-tension used for the calculations (Figure 4.22). Rädler et al. measured pre-tensions of GUVs weakly adhering to a BSA-passivated glass surface ranging from $10^{-3}$ to $10^{-1} \mathrm{mN} / \mathrm{m}$ by reflectometric interference microscopy (RICM). For the calculation of the lateral membrane tension in this thesis an intermediate pre-tension of $10^{-2} \mathrm{mN} / \mathrm{m}$, an area compressibility modulus of $265 \mathrm{mN} / \mathrm{m}$ and a bending rigidity of $21 K_{\mathrm{B}} T$ were used.167, 169]

The calculated mean lateral tensions of GUVs lacking $\mathrm{PIP}_{2}$ (DOPC/DOPE/capbiotin-DOPE/Atto488-DPPE, 67:30:2:1) adhering to an avidin-coated glass surface range from $0.01 \mathrm{mN} / \mathrm{m}$ at $1 \mathrm{~mm} \mathrm{Mg}^{2+}$ to $0.3 \mathrm{mN} / \mathrm{m}$ at $6 \mathrm{mM} \mathrm{Mg}^{2+}$. 
Lateral membrane tensions for $\mathrm{PIP}_{2}$-containing GUVs (DOPC/DOPE/capbiotin-DOPE/Atto488-DPPE/PIP 2 , 66.2:30:2:1:0.8) were higher $(0.08 \mathrm{mN} / \mathrm{m}$ to $1.02 \mathrm{mN} / \mathrm{m}$ ) and a lower $\mathrm{Mg}^{2+}$ concentration was sufficient to promote the adhesion required to result in tension. At low $\mathrm{Mg}^{2+}$ concentrations the membrane tension was only slightly higher than the pre-tension used for the calculation $\left(\sigma_{0}=0.01 \mathrm{mN} / \mathrm{m}\right)$. Therefore, very weak interactions of the GUVs and the avidincoated glass surface were measured. The excess area stored in undulations compensates the area dilatation caused by adhesion to the surface which results in low lateral membrane tensions at low $\mathrm{Mg}^{2+}$ concentrations.
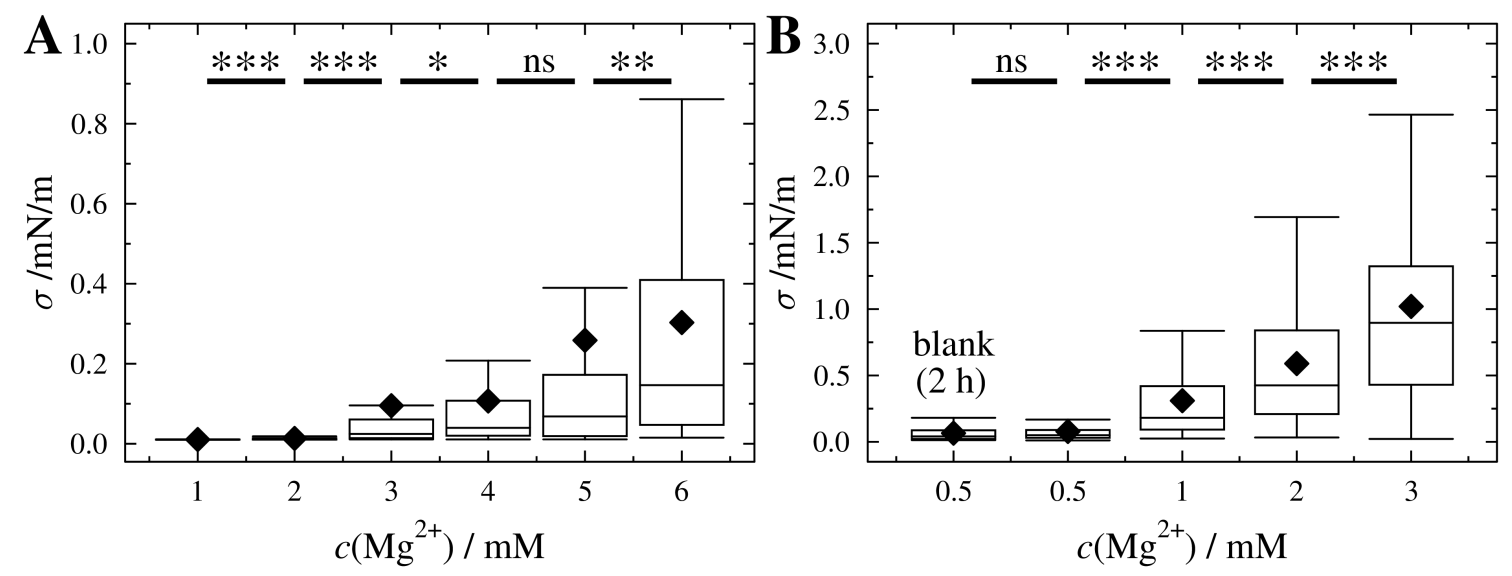

Figure 4.25: Lateral membrane tension of GUVs adhering to an avidin-coated glass surface at different $\mathrm{Mg}^{2+}$ concentrations. Lower tensions were measured for GUVs lacking the receptor lipid $\mathrm{PIP}_{2}$ (A, DOPC/DOPE/cap-biotin-DOPE/Atto488-DPPE, 67:30:2:1) compared to those containing $0.8 \% \mathrm{PIP}_{2}$ (B, DOPC/DOPE/cap-biotin-DOPE/Atto488DPPE/PIP $2,66.2: 30: 2: 1: 0.8)$. Statistical Mann-Whitney $U$ test: ${ }^{* *}: p<0.001,{ }^{* *}: p<0.01$, *: $p<0.05$, ns: not significant.

The adhesion energy of vesicles adhering to a solid support is calculated based on the vesicles' geometry. In the strong adhesion limit, the free energy $F$ is only a function of adhesion energy and elastic energy. Minimizing the free energy $F$ of vesicles strongly adhering to a substrate yields the following condition:

$$
\frac{\mathrm{d} F}{\mathrm{~d} A_{\mathrm{i}}}=K_{A} \frac{\Delta A}{A_{\mathrm{v}}} \frac{\mathrm{d} A}{\mathrm{~d} A_{\mathrm{i}}}-\gamma_{\mathrm{ad}}=0
$$

The solution of equation 4.24 is given by Ramachandran et al., who calculated the adhesion energy of two strongly adhering vesicles (equation 4.25). 159] The adhesion energy of the membrane to the substrate was calculated using the geometry of the 
individual vesicles adhering to the substrate (equation 4.25).

$$
\left.\begin{array}{rl}
\gamma_{\mathrm{ad}}= & K_{A}\left(1-\sqrt{1-\left(\frac{r_{\mathrm{i}}}{r_{\mathrm{ad}}}\right)^{2}}\right) \cdot \\
& \left(\frac{3-\sqrt{1-\left(\frac{r_{\mathrm{i}}}{r_{\mathrm{ad}}}\right)^{2}}}{\left(2\left(1+\sqrt{1-\left(\frac{r_{\mathrm{i}}}{r_{\mathrm{ad}}}\right)^{2}}\right)^{1 / 2}\left(2-\sqrt{1-\left(\frac{r_{\mathrm{i}}}{r_{\mathrm{ad}}}\right)^{2}}\right)\right)^{2 / 3}}-1\right.
\end{array}\right)
$$

Adhesion energies ranging from 0.8 to $780 \mu \mathrm{J} / \mathrm{m}^{2}$ were calculated and are shown in Figure 4.26 .
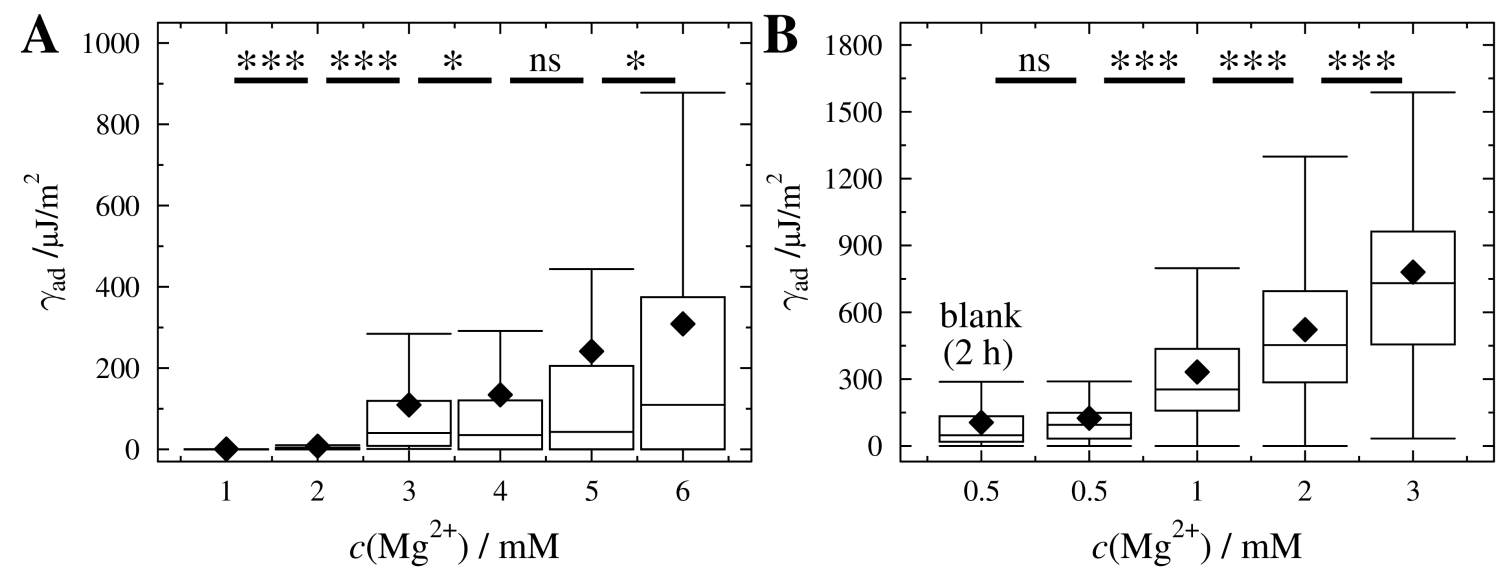

Figure 4.26: Adhesion energies of GUVs adhering to an avidin-coated glass surface at different $\mathrm{Mg}^{2+}$ concentrations. Lower adhesion energies were measured for GUVs lacking the receptor lipid $\mathrm{PIP}_{2}$ (A, DOPC/DOPE/cap-biotin-DOPE/Atto488-DPPE, 67:30:2:1) compared to those containing $0.8 \% \mathrm{PIP}_{2}$ (B, DOPC/DOPE/cap-biotin-DOPE/Atto488DPPE/PIP $2,66.2: 30: 2: 1: 0.8)$. Statistical Mann-Whitney $U$ test: ${ }^{* *}: p<0.001,{ }^{* *}: p<0.01$, *: $p<0.05$, ns: not significant.

The adhesion strength can be divided into a weak and a strong adhesion regime. [174] Adhesion energies smaller than $1 \mu \mathrm{J} / \mathrm{m}^{2}$ are typical for weak adhesion where energetic contributions from membrane bending have to be considered. 158, 175] Those small adhesion energies were only measured for GUVs without $\mathrm{PIP}_{2}$ (DOPC/DOPE/cap-biotin-DOPE/Atto488-DPPE, 67:30:2:1) adhering to the substrate in the presence of $1 \mathrm{~mm} \mathrm{Mg}{ }^{2+}$. At higher $\mathrm{Mg}^{2+}$ concentrations of 2 to $6 \mathrm{~mm}$ 
adhesion energies larger than $1 \mu \mathrm{J} / \mathrm{m}^{2}$ were calculated for the $\mathrm{PIP}_{2}$-free GUVs. For GUVs containing $0.8 \% \mathrm{PIP}_{2}$ larger contact areas and therewith higher adhesion energies ranging from $125 \mu \mathrm{J} / \mathrm{m}^{2}$ at $0.5 \mathrm{~mm} \mathrm{Mg}{ }^{2+}$ to $780 \mu \mathrm{J} / \mathrm{m}^{2}$ at $3 \mathrm{~mm} \mathrm{Mg} \mathrm{Mg}^{2+}$ were calculated. This proves that strong adhesion was measured for the GUVs containing $0.8 \% \mathrm{PIP}_{2}$ at all $\mathrm{Mg}^{2+}$ concentrations. Hence, energetic contributions from membrane bending are negligible and the free energy is only dependent on the elastic and adhesion energy (equation 4.9.

The observed vesicle geometry of a spherical cap is typical for strong adhesion. The calculated adhesion energies for $\mathrm{PIP}_{2}$-free GUVs at $\mathrm{Mg}^{2+}$ concentrations larger than $1 \mathrm{~mm}$ and of GUVs containing PIP 2 at all given $\mathrm{Mg}^{2+}$ concentrations were larger than $1 \mu \mathrm{J} / \mathrm{m}^{2}$. This proves the applicability of the theoretical framework described in Section 4.5.1.

Even though the ratios of $r_{\mathrm{i}} / r_{\mathrm{ad}}$ of $\mathrm{PIP}_{2}$-containing GUVs were relatively large compared to the GUVs lacking $\mathrm{PIP}_{2}$, the resulting lateral tensions $(\sigma>0.08 \mathrm{mN} / \mathrm{m})$ were much smaller than the tension of pore-spanning membranes $(\approx 1-3 \mathrm{mN} / \mathrm{m})$ and within the tension range measured in cells. [29, 33, 35, 119],139] The possibility to tune membrane tension of GUVs in a range similar to that of cells, enables to analyze and quantify the ENTH activity as a function of the lateral membrane tension. Additionally, experiments with GUVs allow quantification of protein surface densities and therewith the correlation of protein activity to the number of proteins on the surface as well as to the lateral membrane tension.

\subsection{Quantification of fluorophore surface densities}

\subsubsection{Distribution of $\mathrm{PIP}_{2}$ in GUVs}

$\mathrm{PIP}_{2}$ is involved in many physiological processes such as cytoskeleton remodeling, ion channel regulation and vesicle trafficking including exo- and endocytosis. It is discussed that regulation of the different processes in cells is achieved by creating local enrichments of $\mathrm{PIP}_{2}$. Among others, clustering can be induced by hydrogen bonds, portioning into raft-like domains and electrostatic interactions. [176] It is also discussed that $\mathrm{PIP}_{2}$-clustering can prevent $\mathrm{PIP}_{2}$ from interacting with certain proteins. In addition to clustering, interactions of $\mathrm{PIP}_{2}$ and the avidin surface are conceivable and could lead to $\mathrm{PIP}_{2}$-enriched and depleted regions. 
The size of the contact area of GUVs adhering to an avidin-coated glass surface has been quantified as a function of the $\mathrm{Mg}^{2+}$ concentration and the resulting lateral tension was calculated. GUVs adhering to an avidin-coated glass surface were imaged by SDCLM to check for an inhomogeneous distribution of $\mathrm{PIP}_{2}$ in the GUVs caused by $\mathrm{Mg}^{2+} .10 \%$ of the $\mathrm{PIP}_{2}(0.08 \%$ of total lipids $)$ was replaced by Bodipy-TMR-PIP 2 . A fluorescent probe with the fluorophore at the fatty acid chain was chosen since $\mathrm{PIP}_{2}$-clustering caused by electrostatic interactions involves head group-cation interactions and dehydration of the head group. 177-179] A homogeneous fluorescence of Bodipy-TMR-PIP 2 was imaged for GUVs (DOPC/DOPE/cap-biotin-DOPE/PIP 2 /Bodipy-TMR-PIP 2 /Atto488-DPPE, 66.5:30:2:0.9:0.1:0.5) in sucrose buffer without $\mathrm{Mg}^{2+}$ indicating the lack of clustering of Bodipy-TMR-PIP 2 (Figure 4.27 A). Likewise, an increased fluorescence intensity near the substrate surface caused by an accumulation of Bodipy-TMR$\mathrm{PIP}_{2}$ was not detected since the absence of $\mathrm{Mg}^{2+}$ ions only led to weak interactions of the biotinylated lipids with the avidin molecules. The experiment was repeated, changing sucrose buffer to PBS to check if $\mathrm{PIP}_{2}$-accumulation of GUVs adhering to the avidin-coated surface occurs. Hyper-osmotic conditions were used to create membrane excess area and to facilitate adhesion of GUVs (DOPC/DOPE/capbiotin-DOPE/PIP 2 /Bodipy-TMR-PIP 2 /Atto488-DPPE, 66.8:30:2:0.9:0.1:0.2) to the avidin surface in the absence of $\mathrm{Mg}^{2+}$ ions (Figure $4.27 \mathrm{~B}$ ). No clustering or accumulation of Bodipy-TMR-PIP 2 was observed in the absence of $\mathrm{Mg}^{2+}$ ions regardless of the size of the vesicles' contact area. A high $\mathrm{Mg}^{2+}$ concentration was used to create strong adhesion of GUVs (DOPC/DOPE/cap-biotin-DOPE/PIP 2 /BodipyTMR-PIP 2 /Atto488-DPPE, 66.8:30:2:0.9:0.1:0.2) to the avidin-coated surface and to facilitate clustering. Similar to the experiments with $\mathrm{Mg}^{2+}$-free sucrose buffer and PBS, no increased fluorescence intensity of Bodipy-TMR-PIP 2 near the substrate surface was detected at a $\mathrm{Mg}^{2+}$ concentration of $5 \mathrm{~mm}$ which would indicate an accumulation of $\mathrm{PIP}_{2}$. A homogeneous fluorescence of Bodipy-TMR-PIP 2 was imaged showing the corresponding homogeneous distribution of $\mathrm{PIP}_{2}$ (Figure 4.27 C). The absence of macroscopic PIP $2_{2}$-clusters in the presence of $\mathrm{Mg}^{2+}$ ions allowed to analyze ENTH tubulation activity as a function of the lateral membrane tension. The homogeneous $\mathrm{PIP}_{2}$-distribution was also a prerequisite for quantification of protein surface densities. 

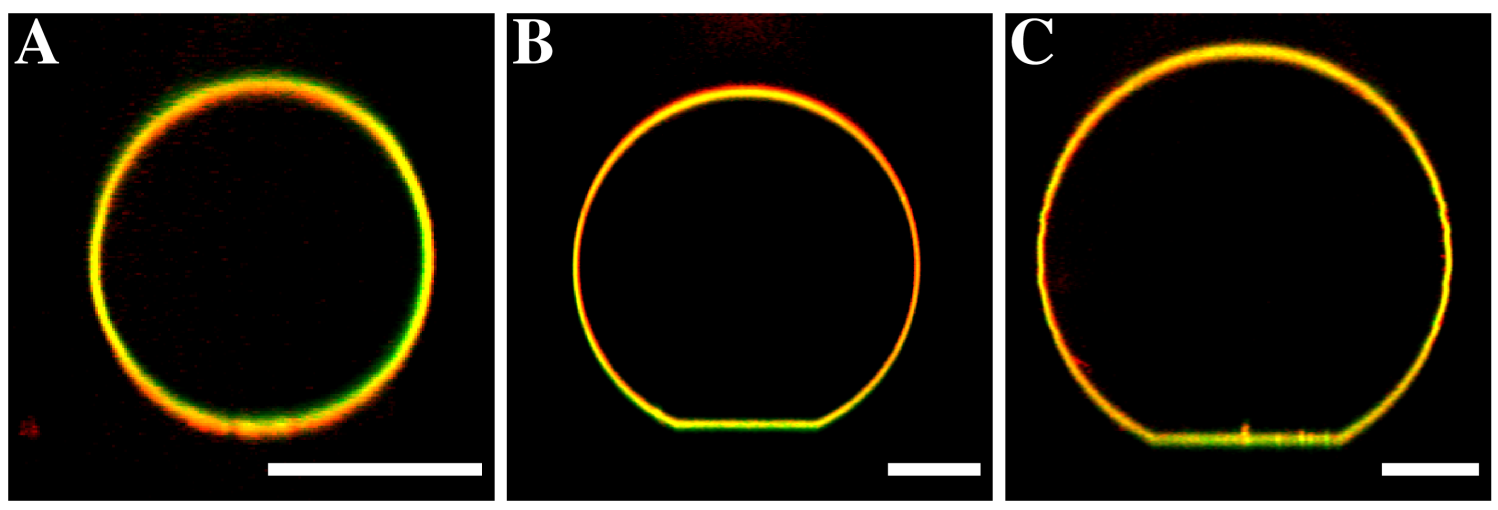

Figure 4.27: Overlay of SDCLM z-profiles of GUVs labeled with Atto488-DPPE (green) and Bodipy-TMR-PIP 2 (red) on an avidin-coated glass surface imaged for different buffers and $\mathrm{Mg}^{2+}$ concentrations. Colocalization of homogeneous fluorescence signals of Atto488-DPPE and Bodipy-TMR-PIP 2 was imaged regardless of the chosen buffer and the presence or absence of $\mathrm{Mg}^{2+}$. A: GUV (DOPC/DOPE/capbiotin-DOPE/PIP 2 /Bodipy-TMR-PIP 2 /Atto488-DPPE, 66.5:30:2:0.9:0.1:0.5) in sucrose buffer without $\mathrm{Mg}^{2+}$. B: GUV (DOPC/DOPE/cap-biotin-DOPE/PIP 2 /Bodipy-TMR$\mathrm{PIP}_{2}$ /Atto488-DPPE, 66.8:30:2:0.9:0.1:0.2) in PBS. C: GUV (DOPC/DOPE/cap-biotinDOPE/PIP 2 /Bodipy-TMR-PIP 2 /Atto488-DPPE, 66.8:30:2:0.9:0.1:0.2) in sucrose buffer with $5 \mathrm{mM} \mathrm{Mg}^{2+}$. Scale bars: $10 \mu \mathrm{m}$.

\subsubsection{Intensity calibration}

Experiments with fluorescent labeled protein have been used to prove protein activity and localization. However, in the last years the focus of interest shifted to quantifying the amount of protein required to induce a certain activity and to correlate the activity to the protein concentration at the membrane. This is especially important as protein crowding slows down the diffusion dynamics in lipid bilayers compared to artificial membranes. [180] Additionally, a high protein density is discussed to contribute to the gerneration of membrane curvature. 129 To answer the questions regarding protein surface concentrations new calibration techniques were developed and applied. Quantification is achieved by stepwise photobleaching of fluorophores to the single molecule level or by using a calibration standard. The calibration standard is often a planar surface yielding a dye number per area or is based on single molecules. ${ }^{[181-183]}$ For quantification a homogeneous distribution of fluorophores inside the region of interest is essential. [176]

To quantify absolute protein concentrations, an intensity calibration using a calibration standard was performed. To avoid a Förster resonance energy transfer 
from the fluorophore attached to the protein to the fluorophore molecules in the vesicle membrane, the fluorophore with the longer emission wavelength was used for protein labeling. The vesicle membrane was labeled with Atto488-DPPE, a bright and very stable dye. If the protein had been labeled with Atto488 or a dye with similar spectral properties, a dye with shorter excitation and emission wavelengths would have been necessary for labeling the GUV membrane. Those dyes are dim and sensitive to photobleaching and would have been too unstable for long time imaging. Therefore, ENTH was labeled with Atto594-maleimide. GUVs doped with Atto594-labeled lipids were used to create a calibration standard. $Z$-stacks of GUVs (DOPC/DOPE/cap-biotin-DOPE/Atto594-DPPE, 68- $x: 30: 2: x$, $x=0.02-0.3, n=74)$ with known fluorophore content $(\mathrm{x} \%)$ were prepared by electro-formation (Section 3.5.1) and imaged by SDCLM. Before quantifying the fluorescence intensities a dark image resulting from the camera offset intensity was subtracted and inhomogeneous illumination resulting from the Gaussian laser profile was corrected (Section 3.8.1).

When utilizing calibration standards it is beneficial to use planar membranes since the measured intensity can be easily converted into a protein density. The membrane of a GUV was approximated as planar since the intensity was measured in a very small area. Intensity quantification was performed using a line profile evaluation and an axial profile evaluation of $z$-stacks of GUVs. For the line profile evaluation the mean intensity of a five pixel broad profile along the $x$ coordinate was quantified (Figure 4.28 A, yellow). Averaging of five neighbored line profiles increased the signal-to-noise-ratio of the intensity profile. The intensity profile (Figure 4.28 B) displayed two peaks indicating the position of the vesicle membrane fitted separately using a Gaussian function. The amplitude of the Gaussian function yielded the fluorophore intensity. ${ }^{[84}$ An intensity profile in $z$-direction (Figure $4.28 \mathrm{C}$ ) was measured in the GUV center (Figure 4.28 A, blue) and quantified.

The qualitative course of the axial profile resembled the one of the line profile. Two peaks were measured indicating the position of the upper and lower GUV membrane but only the peak resulting from the upper vesicle membrane was quantified since the vesicles were adhered to a surface possibly leading to partial quenching of the fluorescence. The extension of the point spread function is larger in axial direction (Section 3.4.1) resulting in broader peaks for the axial profile compared to the line profile evaluation. A laser power of $10 \%$ and an exposure 
time of $150 \mathrm{~ms}$ were used for image acquisition to minimize photobleaching while maintaining a sufficiently high frame rate. A filter (ZET $561 \mathrm{NF}$ ) blocking only the excitation laser was used to detect the entire emission spectrum of the fluorophore therewith detecting the maximum intensity possible.
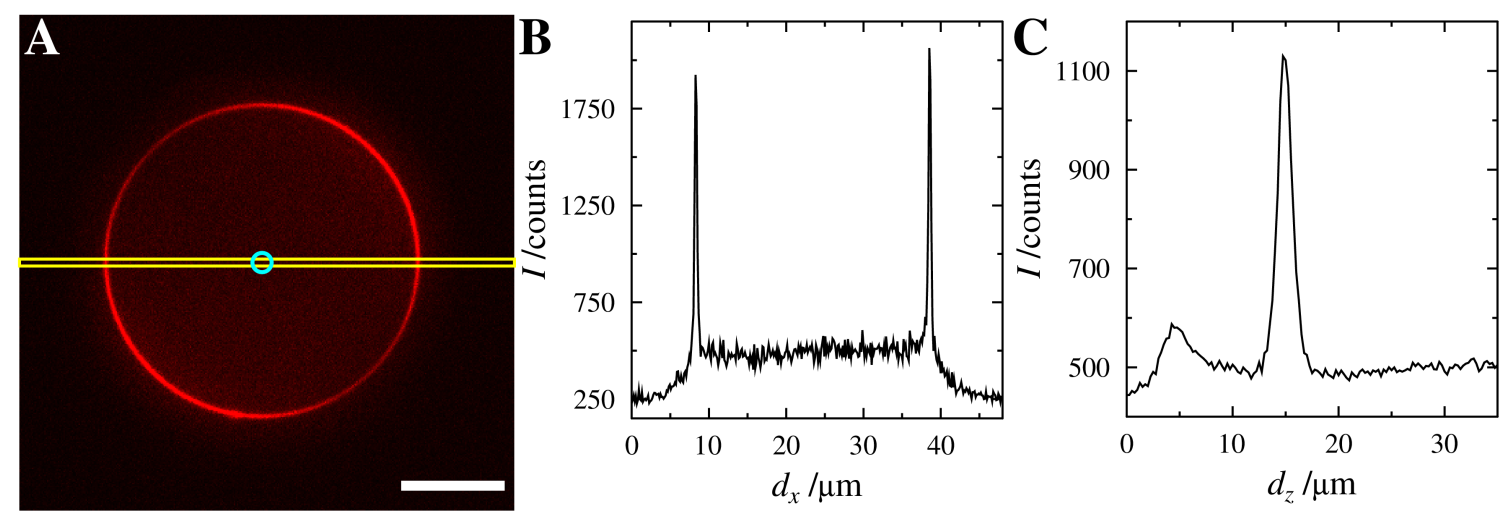

Figure 4.28: A: Overlay of a SDCLM image of a GUV (DOPC/DOPE/cap-biotinDOPE/Atto594-DPPE, 67.7:30:2:0.3) and regions used for the line profile (yellow) and the axial profile (blue) intensity readout. Representative course of a line profile (B) and an axial profile $(\mathbf{C})$.

The mean fluorophore intensities of the vesicles obtained with the line profile and the axial profile evaluation are shown in Figure 4.29. Data were fitted linearly resulting in an intensity per percent Atto594 of 3745 counts for the axial profile quantification and 9025 counts for the line profile evaluation. A correlation coefficient of $R^{2}=0.99$ proved the expected linear correlation between intensity and fluorophore content. The relative position of the data points was identical for both evaluation strategies since the same vesicles were evaluated. The absolute fluorescence intensities were lower for the quantification using the axial profile. The point spread function of a fluorophore has a wider extent in axial than in lateral direction which results in a larger extent of the focal volume in $z$-direction. Therefore, more fluorophores per pixel were excited at the membrane position for the line profile yielding a higher signal. Additionally, the axial profile evaluation is strongly affected by pinhole crosstalk. Pinhole crosstalk, caused by detection of out-of-focus fluorescence, increases the background fluorescence and leads to misidentification of fluorophore densities. For these reasons the line profile evaluation was chosen for quantification of the surface ENTH density. 

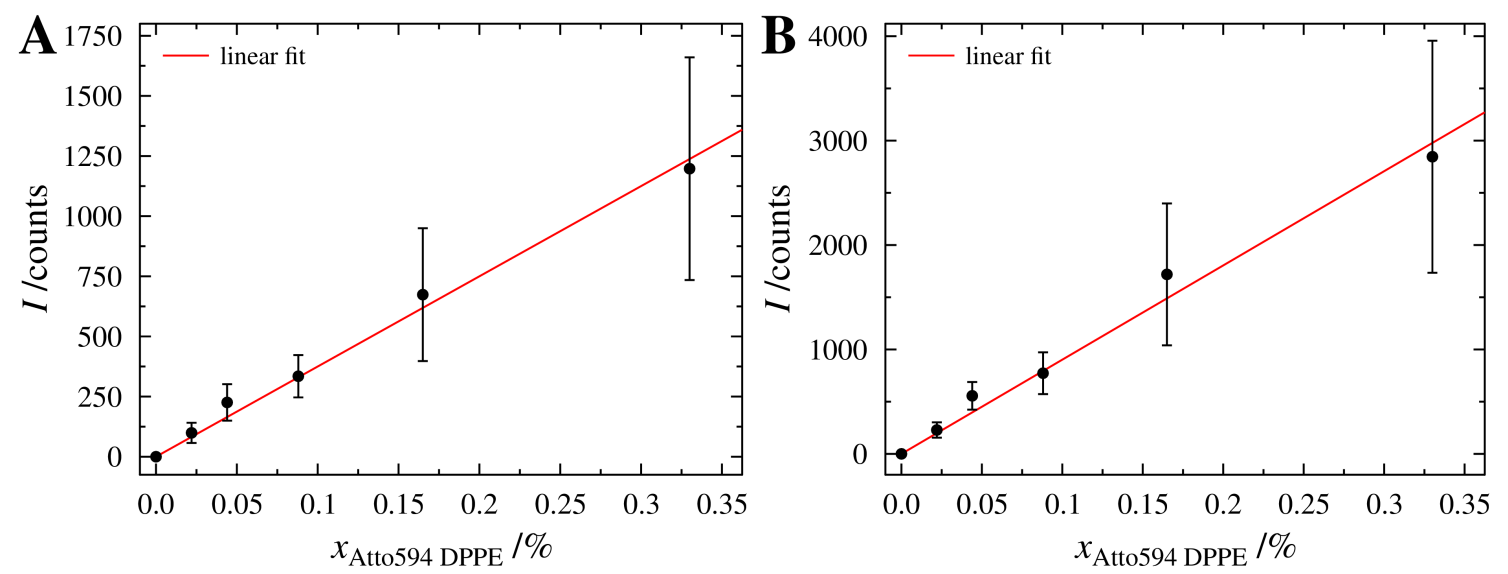

Figure 4.29: Plot of absolute fluorescence intensity of GUVs (DOPC/DOPE/cap-biotinDOPE/Atto594-DPPE, 68-x:30:2:x, $x=0.02-0.3)$ as a function of the fluorophore content (x) obtained by axial profile (A) and line profile (B) evaluation.

The slope of the linear fit, the area per lipid and the fraction of dye molecules were used to calculate the fluorescence intensity per fluorophore molecule within the chosen exposure time. Within one pixel $(133 \mathrm{~nm} \times 133 \mathrm{~nm}) 2.64 \cdot 10^{4}$ lipids were located assuming an area per DOPC molecule of $0.67 \mathrm{~nm}^{2}$.184 185] Assuming $1 \%$ of fluorescently labeled lipids, 264 fluorophores are located within one pixel. Using the intensity obtained by the linear regression (9025 counts/\% Atto594DPPE), this translates to 34.2 counts per fluorophore molecule within the chosen exposure time of $150 \mathrm{~ms}$. The intensity of the fluorescently labeled protein $\left(I_{\text {protein }}\right)$ bound to the lipid bilayer can then be converted to a protein density ( $x_{\text {protein }}$ ) using the intensity per fluorophore obtained ( $I_{\text {fluorophore}}$ ) from the calibration, the correction factor $F$ accounting for the different chemical environments of the fluorophore at the protein and lipid, the degree of labeling ( $D O L)$ and the size of a pixel (equation 4.26). Imaging solutions of SUVs and ENTH with identical molar concentration of the fluorophore yielded a correction factor $F$ of $F=I_{\text {Atto594-DPPE }} / I_{\text {ENTH-Atto594 }}=1.20 \pm 0.05$.

$$
x_{\text {protein }}=\frac{I_{\text {protein }} \cdot F}{I_{\text {fluorophore }} \cdot A_{\text {pixel }} \cdot D O L}
$$




\subsubsection{Binding of ENTH-Atto594 to giant unilamellar vesicles}

ENTH was labeled with Atto594-maleimide as described in Section 3.3.1 resulting in a degree of labeling of $65 \%$. To GUVs with (DOPC/DOPE/cap-biotinDOPE/Atto488-DPPE/PIP 2 , 66.2:30:2:1:0.8) and without PIP 2 (DOPC/DOPE/capbiotin-DOPE/Atto488-DPPE, 67:30:2:1) different concentrations of ENTH-Atto594 were added. Binding of ENTH to the vesicles was only observed in the presence of $\mathrm{PIP}_{2}$ resulting in a colocalization (Figure $4.30 \mathrm{~A}$, yellow) of the membrane fluorescence (Figure 4.30 A, green) and ENTH fluorescence (Figure 4.30 A, red) which was not detectable if the GUVs were lacking the receptor lipid $\mathrm{PIP}_{2}$ (Figure 4.30 B). Intensities of ENTH bound to the GUV membrane were quantified using the line profile evaluation described in Section 4.6.2. The protein fluorescence intensities measured were plotted vs. the ENTH concentration in solution (Figure $4.30 \mathrm{C}$ ). By fitting equation 4.1 to the data, a dissociation constant of $0.5 \pm 0.2 \mu \mathrm{M}$ was determined being similar to the dissociation constants obtained with RIfS and SPR spectroscopy for ENTH binding to POPC and POPC/POPE bilayers (Section 4.1.3).
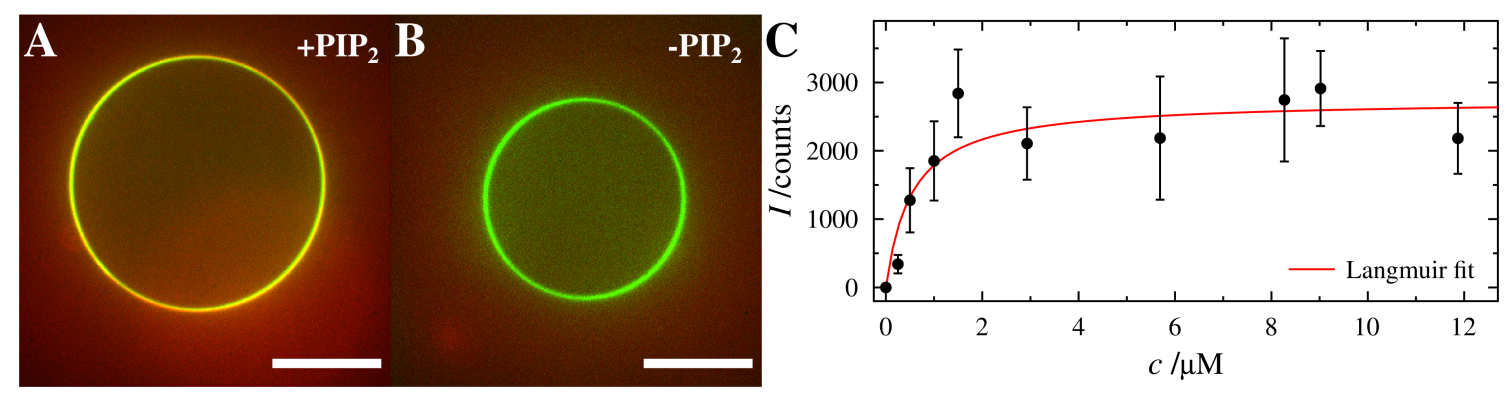

Figure 4.30: A+B: Overlay of confocal fluorescence micrographs of ENTH-Atto594 (red, $c_{\mathrm{ENTH}}=1 \mu \mathrm{M}$ ) bound to a GUV membrane doped with Atto488-DPPE (green, DOPC/DOPE/cap-biotin-DOPE/Atto488-DPPE/PIP $2,66.2: 30: 2: 1: 0.8)$ in the presence (A) or absence (B) of PIP 2 . Binding of ENTH-Atto594 to the GUV membrane was observed in the presence of $\mathrm{PIP}_{2}$. Scale bars: $20 \mu \mathrm{m}$. C: Binding isotherm of ENTH-Atto594 binding to GUVs (DOPC/DOPE/cap-biotin-DOPE/Atto488-DPPE/PIP 2 , 66.2:30:2:1:0.8). Data were fitted using the Langmuir adsorption model. A dissociation constant of $0.5 \pm 0.2 \mu \mathrm{M}$ was determined.

In addition to the specific binding of ENTH-Atto594 to $\mathrm{PIP}_{2}$-containing membranes unspecific binding to the avidin-coated substrate surface was detected (Figure 4.31A). Higher intensities of ENTH-Atto594 immobilized on the surface than bound to the vesicle membrane were detected. The entire glass surface was 
covered with ENTH-Atto594 except for the region of the vesicles' contact area. Nevertheless, the protein concentration in solution was approximately constant since the fraction of ENTH molecules binding to the substrate surface is small compared to the total number in solution $(\approx 2 \%$ assuming a petri dish with a radius of $1.5 \mathrm{~cm}$ and an area of the ENTH footprint of $\left.16 \mathrm{~nm}^{2}\right)$. [8, [9]

A decreased stability of ENTH at ambient temperature after labeling and the formation of large protein clusters which were precipitating after about 30 min was observed. The clusters grew in size as a function of time and stuck to the substrate surface and the GUVs disturbing the intensity quantification by overexposing the ENTH-Atto594 intensity bound to the GUV membrane. Cluster formation was not only detected by fluorescence microscopy, it was also visible by naked eye after a longer period of time ( 2 to $3 \mathrm{~h}$ ). No protein clusters were observed when using non-labeled ENTH. To answer the question of the fluorophore being responsible for the decreased protein stability, UV/VIS measurements were conducted. To slow down the precipitation process ENTH-Atto594 was stored at $4{ }^{\circ} \mathrm{C}$ and spectra were measured every 3 to 4 days. Even though relatively large fluctuations of the ENTH concentration were measured, no clear decrease in concentration was observed (Figure 4.31 B). In contrast to the ENTH concentration, the degree of labeling rapidly diminished as a function of time from about $70 \%$ to $35 \%$ (Figure $4.31 \mathrm{C}$ ).
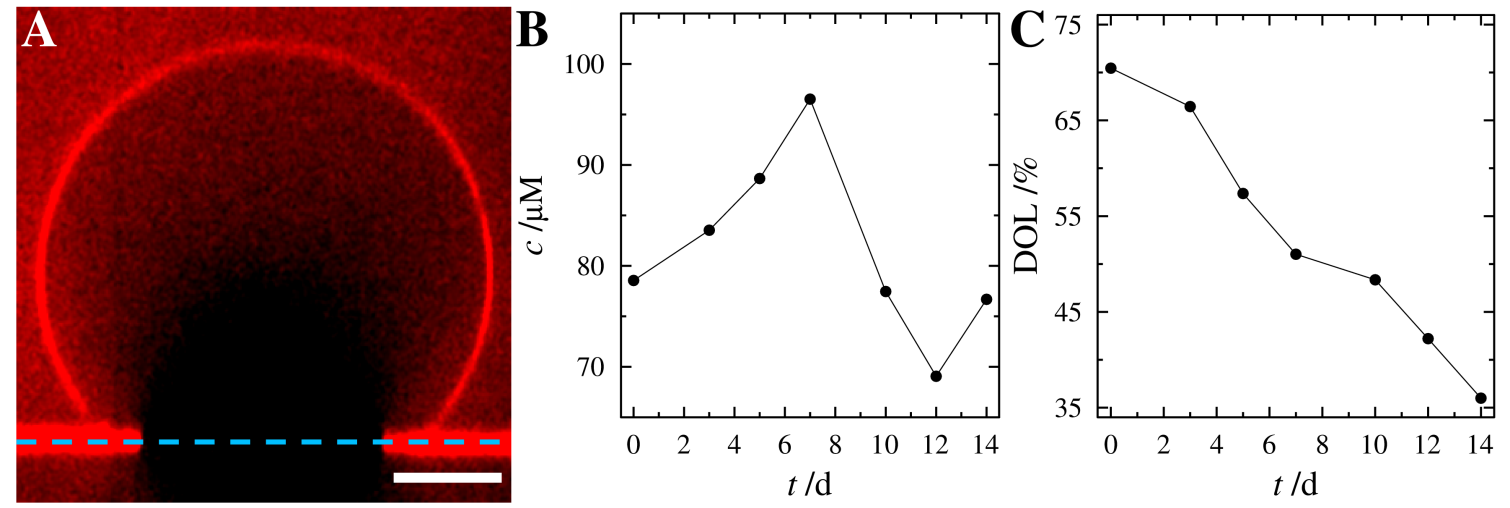

Figure 4.31: A: Z-profile of an adhered GUV (DOPC/DOPE/cap-biotin-DOPE/Atto488DPPE/PIP $2,66.2: 30: 2: 1: 0.8)$ after ENTH-Atto594 addition $(c=1 \mu \mathrm{M})$. Specific binding of ENTH-Atto594 to the GUV membrane and unspecific binding to the substrate surface (substrate position: dotted blue line) was observed. Scale bar: $10 \mu \mathrm{m}$. B: Large fluctuations of the total concentration of a mixture of ENTH and ENTH-Atto594 were measured as a function of time. C: Degree of labeling (DOL) of a mixture of ENTH and ENTH-Atto594 rapidly decreasing as a function of time. Scale bar: $10 \mu \mathrm{m}$. 
This led to the conclusion that the labeled protein is precipitating and that the attached fluorophore decreases the protein stability. To exclude changes in protein stability or activity, non-labled ENTH was used for further experiments.

\subsection{Interaction of ENTH with adhered GUVs as a function of the lateral membrane tension}

\subsubsection{ENTH binding to adhered GUVs}

To analyze changes in vesicle geometry and morphology and to compare the results to those obtained on protruded pore-spanning membranes (Section 4.3) ENTH was added to GUVs strongly adhering to an avidin-coated glass surface in sucrose buffer containing $2 \mathrm{~mm} \mathrm{Mg}^{2+}$. A mean ratio of $r_{\mathrm{i}} / r_{\mathrm{ad}}$ of 0.59 corresponding to a lateral tension of $0.59 \mathrm{mN} / \mathrm{m}$ (Section 4.5.2) was measured for GUVs $(n=95)$ in sucrose buffer containing $2 \mathrm{~mm} \mathrm{Mg}{ }^{2+}$. This tension is significantly lower compared to the tension of pore-spanning membranes $(1$ to $3 \mathrm{mN} / \mathrm{m})$. 119, 139, 150] However, it is still a relatively high membrane tension when compared to cells. 29, 33-35] GUVs adhered to an avidin-coated glass surface in sucrose buffer containing $2 \mathrm{~mm}$ $\mathrm{Mg}^{2+}$ did not show vesiculation or tubulation after ENTH addition $\left(c_{\text {ENTH }}=1 \mu \mathrm{M}\right)$ as already observed for protruded pore-spanning membranes with a membrane tension of about $2 \mathrm{mN} / \mathrm{m}$. Most likely the median membrane tension of $0.59 \mathrm{mN} / \mathrm{m}$ is still too high and prevents necessary changes in membrane morphology for the formation of highly curved structures such as vesicles or tubules. Instead of forming vesicles or tubes the GUVs started to flatten and to increase their contact area upon addition of ENTH (Figure 4.32). Collapsing vesicles were also observed as a result of ENTH addition, especially when using high laser intensities for imaging. The laser intensity was therefore lowered to image GUVs over a long period of time $(\approx 1.5 \mathrm{~h}$ ). Collapsing GUVs were only observed after a long exposure time with relatively high laser intensity and addition of ENTH. 

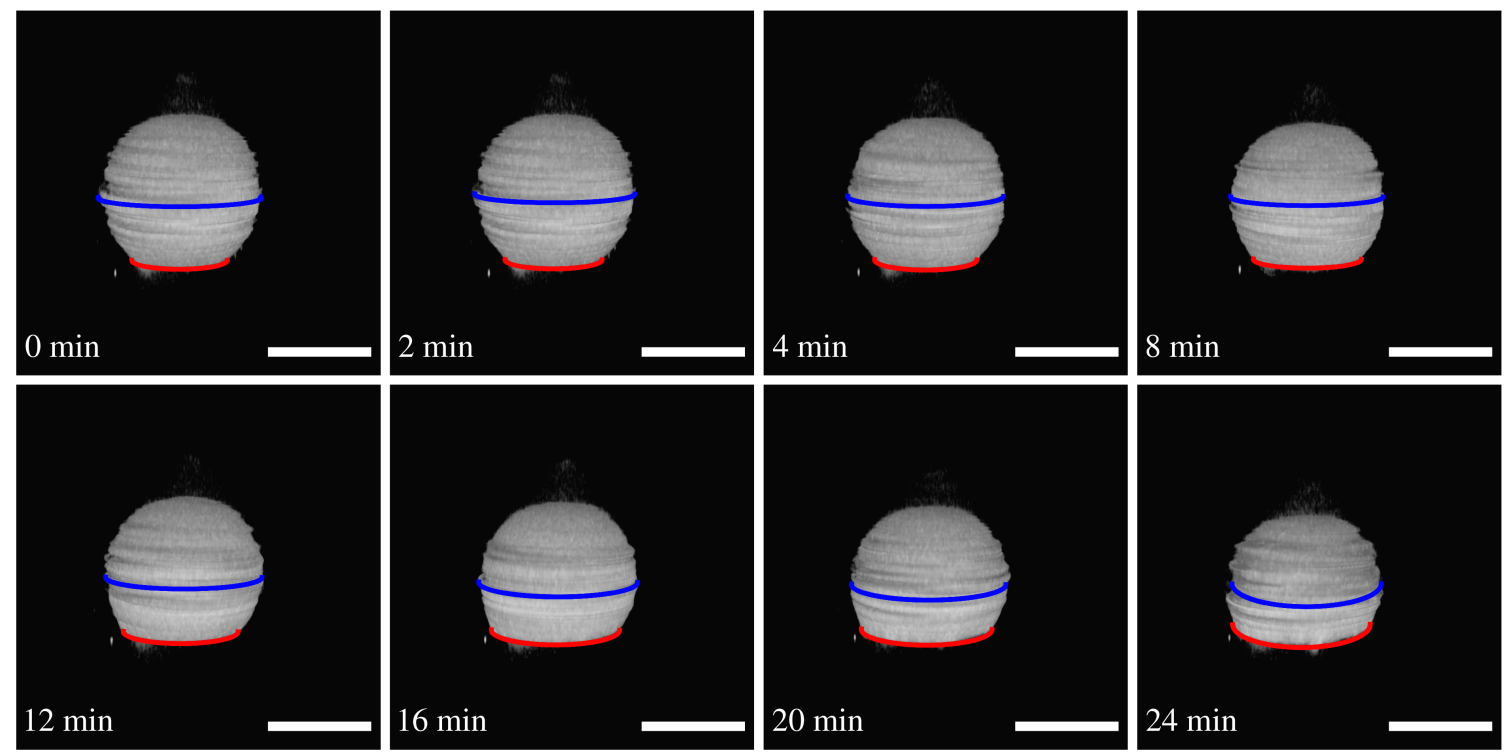

Figure 4.32: Time series of 3D reconstructions of SDCLM images of a GUV (DOPC/DOPE/cap-biotin-DOPE/Atto488-DPPE/PIP 2 , 66.2:30:2:1:0.8) adhering to an avidin-coated glass slide in sucrose buffer containing $2 \mathrm{mM} \mathrm{Mg}^{2+}$. Flattening of the GUV was observed after ENTH $\left(c_{\mathrm{ENTH}}=1 \mu \mathrm{M}\right)$ addition. The size of the contact area increases while the distance between equatorial plane and contact area decreases as a function of time. $t_{0}$ min: start of flattening. Scale bars: $20 \mu \mathrm{m}$.

ENTH is involved in the formation of endocytotic vesicles during clathrin mediated endocytosis and is known to cause tubulation or vesiculation of non-adhering GUVs having a low membrane tension. Therefore, the $\mathrm{Mg}^{2+}$ concentration of the sucrose buffer was lowered to $0.5 \mathrm{~mm}$ to decrease the adhesion strength of the membrane to the substrate resulting in a lower membrane tension. Adhesion of the GUVs (DOPC/DOPE/cap-biotin-DOPE/Atto488-DPPE/PIP 2, 66.2:30:2:1:0.8) in sucrose buffer containing $0.5 \mathrm{mM} \mathrm{Mg}^{2+}$ led to the formation of contact areas with a mean ratio of $r_{\mathrm{i}} / r_{\mathrm{ad}}$ of 0.46 corresponding to a mean lateral tension of $0.08 \mathrm{mN} / \mathrm{m}$ (Section 4.5.2). This tension is about seven times lower compared to vesicles adhering at a $\mathrm{Mg}^{2+}$ concentration of $2 \mathrm{~mm}$ and within the measured tension range for cells. 29, 33,-35]

ENTH $\left(c_{\text {ENTH }}=1 \mu \mathrm{M}\right)$ was added to the adhered vesicles and the geometry was imaged by SDCLM as a function of time. Binding of ENTH to GUVs with low membrane tension led to the formation of tubular structures (Figure 4.33). The appearance of the tubes only at one side of the GUV and the uniform direction of the tubules was attributed to stirring. The size of the vesicle decreased as a function of time until all lipid material was dissipated. In accordance with 
experiments using pore-spanning membranes and GUVs with higher membrane tension no vesiculation was observed. If vesicles had formed, the time resolution ( $2 \mathrm{~min} / z$-stack) would have probably been too low for imaging of detaching vesicles after ENTH addition. As the formation of a vesicle is more complex than the formation of a membrane tube and as no docked vesicles were observed, this indicates that binding of ENTH to GUVs adhering to an avidin-coated glass surface does not result in the formation of vesicles but only in the formation of membrane tubules. [186]
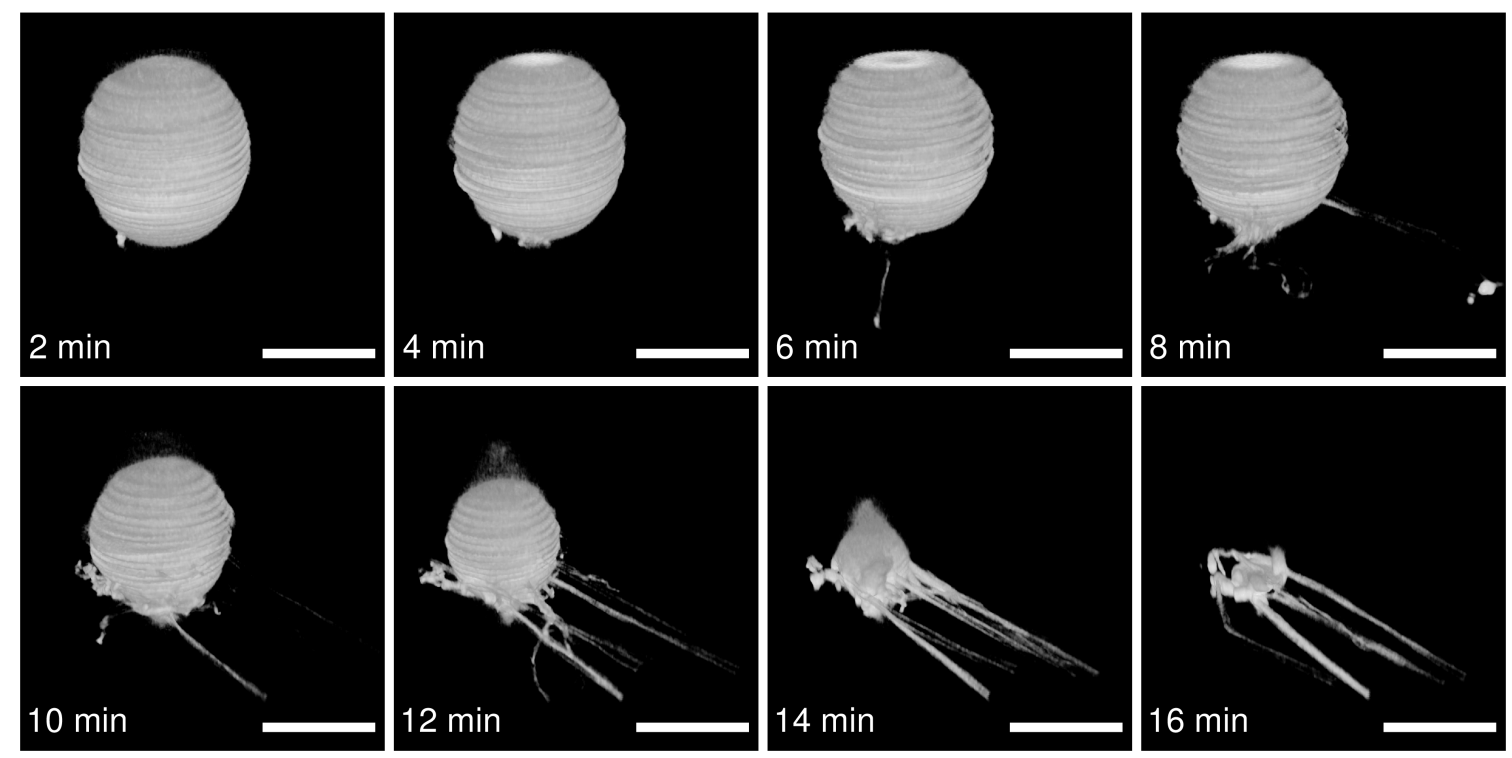

Figure 4.33: Time series of 3D reconstructions of SDCLM images of a GUV (DOPC/DOPE/cap-biotin-DOPE/Atto488-DPPE/PIP $2,66.2: 30: 2: 1: 0.8$ ) adhering to an avidin-coated glass slide in sucrose buffer containing $0.5 \mathrm{~mm} \mathrm{Mg}^{2+}$. Formation of tubular structures is observed after ENTH $\left(c_{\mathrm{ENTH}}=1 \mu \mathrm{M}\right)$ addition. $t_{0}$ min: start of tubulation. Scale bars: $20 \mu \mathrm{m}$.

\subsubsection{Quantification of the change in area as a result of ENTH binding}

Tubulation of vesicles and dissipation of the lipid material was observed for GUVs with low membrane tension $(0.08 \mathrm{mN} / \mathrm{m})$. The vesicle volume was not constant during the tubulation process which allowed to form highly curved structures having a high area-to-volume ratio. This implies that the theoretical 
model described in Section 4.5.1 which allows to correlate the vesicle's geometry to its surface area cannot be applied here since the requirement of constant volume is not fulfilled. Nevertheless, ENTH binding did only cause tubule formation for a fraction of the adhered vesicles. For the majority of the vesicles an increase of the vesicles' contact area was detected indicating their membrane tension still being considerably high preventing tube formation. As GUVs adhering to an avidin-coated glass surface have a certain distribution size and membrane tension (Figure 4.25), presumably only the vesicles with a low membrane tension are tubulated by ENTH.

For GUVs with a high membrane tension $(0.59 \mathrm{mN} / \mathrm{m})$ formation of highly curved structures was not detected. Instead, only flattening of the GUVs after addition of ENTH was observed increasing the vesicles' contact radius. An increase of $r_{\mathrm{i}} / r_{\mathrm{ad}}$ was measured after ENTH binding regardless of the vesicle tension (Figure 4.34). Vesicles not showing any signs of tubulation or vesiculation were selected for the evaluation since these processes would remove lipid material and therefore the theoretical model would not be applicable.

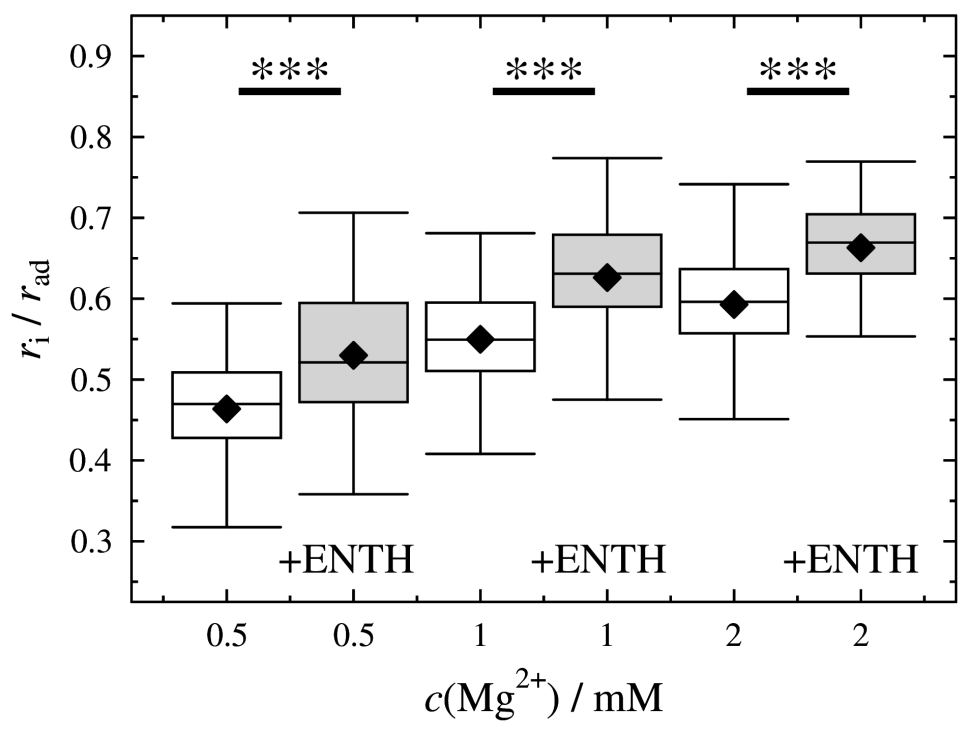

Figure 4.34: $r_{\mathrm{i}} / r_{\mathrm{ad}}$ of GUVs (DOPC/DOPE/cap-biotin-DOPE/PIP $2 /$ Atto488-DPPE, 66.2:30:2:1:0.8) adhering to an avidin-coated glass surface in sucrose buffer with different $\mathrm{Mg}^{2+}$ concentrations measured before and after ENTH addition $\left(c_{\mathrm{ENTH}}=1 \mu \mathrm{m}\right)$. Statistical Mann-Whitney $U$ test: ${ }^{* *}: p<0.001$. 
The changes in area after binding of ENTH are then correlated to the mechanical properties of the lipid bilayer to evaluate whether the increase in area is caused by the size of the inserted helix of ENTH $\delta A_{\mathrm{ENTH}}$ or by altered mechanical properties of the membrane. The free energy after ENTH binding $F_{\mathrm{ENTH}}$ is given by:

$$
F_{\mathrm{ENTH}}=\frac{1}{2} \tilde{K}_{A} \frac{\left(\left(A_{\mathrm{ad}}+\delta A_{\mathrm{ENTH}, \mathrm{ad}}\right)-\left(A_{\mathrm{v}}+\delta A_{\mathrm{ENTH}, \mathrm{v}}\right)\right)^{2}}{\left(A_{\mathrm{v}}+\delta A_{\mathrm{ENTH}, \mathrm{v}}\right)}-A_{\mathrm{i}} \gamma_{\mathrm{ad}},
$$

with $\tilde{K}_{A}$ being the apparent area compressibility modulus after ENTH binding and $\delta A_{\mathrm{ENTH}, \mathrm{v}}$ and $\delta A_{\mathrm{ENTH}, \text { ad }}$ the area increase of the free and adhered vesicle caused by binding of ENTH. Assuming $\delta A_{\mathrm{ENTH}, \mathrm{ad}} \cong \delta A_{\mathrm{ENTH, \textrm {v }}} \cong \delta A_{\mathrm{ENTH}}$ yields:

$$
F_{\mathrm{ENTH}}=\frac{1}{2} K_{A, \mathrm{eff}} \frac{\left(A_{\mathrm{ad}}-A_{\mathrm{v}}\right)^{2}}{A_{\mathrm{v}}}-A_{\mathrm{i}} \gamma_{\mathrm{ad}}
$$

with $K_{A \text {,eff }}$ being the effective area compressibility modulus defined as:

$$
K_{A, \mathrm{eff}}=\tilde{K}_{A} \frac{A_{\mathrm{v}}}{A_{\mathrm{v}}+\delta A_{\mathrm{ENTH}}}
$$

Assuming a constant adhesion energy before and after ENTH binding, the effective area compressibility modulus after ENTH binding can be calculated by rearranging equation 4.25 to:

$$
\begin{aligned}
K_{A, \text { eff }}= & \gamma_{\text {ad }}\left(1-\sqrt{1-\left(\frac{r_{\mathrm{i}}}{r_{\mathrm{ad}}}\right)_{\mathrm{ENTH}}^{2}}\right)^{-1} \cdot \\
& \left(\frac{3-\sqrt{1-\left(\frac{r_{\mathrm{i}}}{r_{\mathrm{ad}}}\right)_{\mathrm{ENTH}}^{2}}}{\left(2\left(1+\sqrt{1-\left(\frac{r_{\mathrm{i}}}{r_{\mathrm{ad}}}\right)_{\mathrm{ENTH}}^{2}}\right)^{1 / 2}\left(2-\sqrt{1-\left(\frac{r_{\mathrm{i}}}{r_{\mathrm{ad}}}\right)_{\mathrm{ENTH}}^{2}}\right)\right)^{2 / 3}}-1\right)^{-1},
\end{aligned}
$$

with $\left(r_{\mathrm{i}} / r_{\mathrm{ad}}\right)_{\mathrm{ENTH}}$ being the ratio of contact radius and radius of the adhered vesicle after ENTH binding. Effective area compressibility moduli of $155 \mathrm{mN} / \mathrm{m}$, $141 \mathrm{mN} / \mathrm{m}$ and $151 \mathrm{mN} / \mathrm{m}$ were calculated for GUVs adhering to an avidin-coated glass surface in sucrose buffer containing $0.5 \mathrm{~mm}, 1 \mathrm{~mm}$ and $2 \mathrm{~mm} \mathrm{Mg}{ }^{2+}$ after ENTH 
addition. The change in area $\delta A_{\mathrm{ENTH}}$ caused by binding and insertion of the ENTH helix into the lipid bilayer required for the reduction of $K_{A}$ to the mean effective area compressibility modulus of $K_{A \text {,eff }}=149 \pm 8 \mathrm{mN} / \mathrm{m}$ was then calculated. Assuming the area compressibility modulus being constant before and after ENTH binding $\left(\tilde{K}_{A}=K_{A}\right)$, the area of the vesicle has to increase by about $78 \pm 9 \%$.

To assess this value, the expected increase in area caused by insertion of the ENTH helix was approximated based on the protein's crystal structure, the $\mathrm{PIP}_{2}$ content in the membrane and the ENTH concentration. Solving equation 4.1 for a protein concentration of $1 \mu \mathrm{M}$ assuming a ratio of ENTH binding to $\mathrm{PIP}_{2}$ of $1: 1$ and a mean dissociation constant of $0.6 \pm 0.1 \mu \mathrm{M}$, as determined by RIfS, SPR spectroscopy and fluorescence intensity quantification, yields that $62.5 \%$ of the total number of receptor lipids having bound an ENTH molecule. For a $\mathrm{PIP}_{2}$ content of $0.8 \%$ in the GUV membrane this translates to $0.50 \%$ of the total lipids binding to an ENTH molecule. The increase in area was then calculated by comparing the area of a $\mathrm{PIP}_{2}$ molecule $\left(0.67 \mathrm{~nm}^{2}\right)$ with the area of the helix. [184, 185] The helix of ENTH has a foot print of $10-30 \%$ of the protein's footprint which is $1.6 \mathrm{~nm}^{2}$ to $4.8 \mathrm{~nm}^{2}$. [8] Thus, the ENTH helix requires about 2.4 to 7.2 times more space than a $\mathrm{PIP}_{2}$ molecule translating to expected area changes of about 1.2 to $3.6 \%$ of the total membrane surface.

As the change in membrane area caused by the insertion of the protein's helix is much smaller than expected considering the area of the helix, it was concluded that binding of ENTH lowers the effective area compressibility modulus. Similar reductions were observered regardless of the lateral membrane tension. Neglecting the small change in area caused by insertion of the helix of ENTH $\left(A_{\mathrm{ENTH}}=0\right)$ the reduction of the effective area compressibility modulus $K_{A \text {,eff }}$ translates into a reduced effective bending rigidity $\kappa_{\text {eff }}$ as shown by equation 4.31 .

$$
\kappa_{\text {eff }}=\frac{K_{A, \text { eff }} d_{\mathrm{hc}}^{2}}{\beta\left(1-v^{2}\right)} .
$$

The parameter $\beta$ accounts for the coupling of the two monolayers, $d_{\mathrm{hc}}$ is the mechanically relevant thickness of the bilayer hydrocarbon core and $v$ the Poisson's ratio. It could be shown that for determining the bending rigidity the mechanically relevant bilayer thickness is the membrane thickness reduced by the head group thicknesses $(\approx 1 \mathrm{~nm})$. ${ }^{[169]}$ Using a thickness of the hydrophobic bilayer core of $d_{\mathrm{hc}}=2.68 \mathrm{~nm}, \beta=24$ for monolayers coupled with friction and a Poisson's ratio of 
$v=0.33$, a bending rigidity before ENTH addition of $\kappa=22 k_{\mathrm{B}} T$ was calculated well matching the bending rigidity determined by Rawicz et al. 169.187. 188] After ENTH addition the mean area compressibility modulus drops to $K_{A \text {,eff }}=149 \pm 8 \mathrm{mN} / \mathrm{m}$ and hence the bending rigidity decreases to $\kappa_{\text {eff }}=12.5 \pm 0.6 k_{\mathrm{B}} T$ resulting in a softening of the membrane.

It was shown that ENTH tubulates a fraction of adhered vesicles with a membrane tension of $0.08 \mathrm{mN} / \mathrm{m}$. The majority of the GUVs flattenend after ENTH addition and thereby increased their contact area. At a higher membrane tension of $0.59 \mathrm{mN} / \mathrm{m}$ tubulation was suppressed and only flattening of vesicles was observed. The increased contact area after ENTH binding was attributed to a decrease of the effective area compressibility modulus and therewith to a reduction of the effective bending rigidity. Softening of membranes after binding of helices was also observed for different helical peptides. $\left.{ }^{189}-191\right]$ In addition to the complex interplay of proteins, changes of mechanical properties such as membrane tension and bending rigidity could be used by cells to regulate the formation of vesicles during endocytosis. 



\section{Discussion}

To answer the question how ENTH binding is influenced by membrane tension experiments with protruded pore-spanning membranes (PSMs) and adhered GUVs were conducted. The dissociation constant was determined by reflectometric interference spectroscopy (RIfS) and surface plasmon resonance (SPR) spectroscopy to estimate the amount of membrane-bound proteins for the given ENTH concentration used in the experiments. The height of the protruded pore-spanning membranes was measured as a function of the osmolarity gradient to calculate the lateral membrane tension using fluorescence microscopy. Binding of ENTH to those membranes resulted in growth and rupture of membranes depending on their lipid composition. To analyze membranes with a lower membrane tension, experiments with giant unilamellar vesicles (GUVs) adhering to an avidin-coated surface were carried out. The membrane tension of the vesicles was tuned by the $\mathrm{Mg}^{2+}$ concentration and allowed to monitor ENTH binding as a function of membrane tension.

\subsection{Binding affinity of ENTH}

The binding affinity of ENTH to its receptor lipid L- $\alpha$-phosphatidylinositol-4,5bisphosphate $\left(\mathrm{PIP}_{2}\right)$ was measured by RIfS, SPR spectroscopy and quantification of fluorescence intensities of labeled protein bound to GUVs using spinning disc confocal laser microscopy (SDCLM). Different membrane topologies and lipid compositions were analyzed and dissociation constants ranging from $0.5 \pm 0.1 \mu \mathrm{M}$ to $0.8 \pm 0.2 \mu \mathrm{M}$ were determined. The experimentally measured dissociation constants are summarized in Table 5.1 . 
Table 5.1: Dissociation constants determined by SPR spectroscopy, RIfS and SDCLM.

\begin{tabular}{cccc}
\hline$K_{\mathrm{D}} / \mu \mathrm{M}$ & Technique & Type of model membrane & Matrix lipids \\
\hline $0.8 \pm 0.2$ & RIfS & solid-supported & POPC \\
$0.5 \pm 0.1$ & SPR & LUV & POPC \\
$0.69 \pm 0.08$ & SPR & LUV & POPC/POPE $(70: 30)$ \\
$0.5 \pm 0.2$ & SDCLM & GUV & DOPC/DOPE $(70: 30)$ \\
\hline
\end{tabular}

In various studies dissociation constants of ENTH binding to $\mathrm{PIP}_{2}$ ranging from $2.6 \mathrm{nM}$ to $0.85 \mu \mathrm{M}$ were measured using isothermal titration calorimetry, SPR spectroscopy and fluorescence microscopy. [13, 47,50,51] Stahelin et al. showed that the dissociation constant of ENTH depends strongly on the experimental conditions such as $\mathrm{PIP}_{2}$ content, buffer composition and $\mathrm{pH}$. ${ }^{[50]}$ Electrostatic interactions have been found to mainly affect association of the ENTH-PIP 2 complex whereas its dissociation is governed by hydrophobic forces. Increasing the $\mathrm{KCl}$ concentration from $0.16 \mathrm{M}$ to $0.5 \mathrm{M}$ increased the dissociation constant from $23 \mathrm{~nm}$ to $330 \mathrm{~nm}$. Decreasing the $\mathrm{PIP}_{2}$ content from $3 \%$ to $0.5 \%$ had a similar effect and increased the dissociation constant from $23 \mathrm{~nm}$ to $250 \mathrm{~nm}$. Rozovsky et al. analyzed the dissociation rate of ENTH as a function of the $\mathrm{PIP}_{2}$ content on a single molecule level and found increasing dissociation rates with decreasing $\mathrm{PIP}_{2}$ content, which supports the results of Stahelin et al. and explains the higher dissociation constants for lower PIP ${ }_{2}$ contents. ${ }^{[192]}$ Binding of ENTH to its receptor lipid has been shown being also dependent on the $\mathrm{pH}$. Hom et al. measured the affinity of ENTH to membranes containing $2 \% \mathrm{PIP}_{2}$ as a function of the $\mathrm{pH}$. The dissociation constant increased from about $10 \mathrm{~nm}$ to $330 \mathrm{~nm}$ when the $\mathrm{pH}$ was increased from 6 to 8. [51] For membranes containing $2 \% \mathrm{PIP}_{2}$ and $15 \%$ 1-palmitoyl-2-oleoyl-sn-glycero3-phospho-L-serine (POPS) a similar $\mathrm{pH}$-dependence was observed but lower dissociation constants ranging from $2.6 \mathrm{~nm}$ at $\mathrm{pH} 6$ to $69 \mathrm{~mm}$ at $\mathrm{pH} 8$ were measured. Those experiments clearly demonstrate that the binding affinity of ENTH is strongly dependent on the experimental conditions and especially on electrostatics. Hereby, the dissociation constants determined in this thesis ranging from $0.5 \pm 0.1 \mu \mathrm{M}$ to $0.8 \pm 0.2 \mu \mathrm{M}$ are in the same range with those measured by other groups.

A variety of endocytotic proteins such as endophilin and epsin are known to not only induce but also to sense membrane curvature. [193, 194] The mean curvature $(C)$ of a membrane is a defined as: 


$$
C=\frac{1}{2}\left(\frac{1}{r_{1}}+\frac{1}{r_{2}}\right)
$$

with $r_{1}$ and $r_{2}$ being the radii along two orthogonal axes. If the radii are larger than the bilayer thickness, membrane curvature is considered as low $\left(C<10^{-4}\right)$ whereas it is considered as high for radii being in the same range as the membrane thickness $\left(C \approx 10^{-1}\right) .[195]$ The preferred type and size of lipid aggregates such as vesicles and micelles is determined by the spontaneous curvature of the lipids. By comparing dissociation constants of ENTH binding to planar and curved membranes only small differences of the dissociation constant were found. The dissociation constant of $0.8 \pm 0.2 \mu \mathrm{M}$ for ENTH binding to planar membranes (POPC/PIP $2,90: 10)$ measured by RIfS was slightly higher than the dissociation constant of ENTH binding to curved vesicles of identical composition with a diameter of $100 \mathrm{~nm}$ measured by SPR spectroscopy $\left(K_{\mathrm{D}}=0.5 \pm 0.1 \mu \mathrm{M}\right)$. It has been reasoned that membrane curvature facilitates the insertion of hydrophobic domains into the membrane and thus may change the dissociation constant. 196, 197] However, it has been shown experimentally that the preferred binding of amphipathic helices to highly curved membranes does not result from higher affinity but from higher density of binding sites. [198] Experiments using liposomes immobilized on a surface showed that the amount of protein bound to the vesicles with different sizes was larger for smaller vesicles. According to the Langmuir-Hill isotherm which extends the Langmuir adsorption model by cooperative interactions, the surface coverage $\theta$ is a function of the surface saturation coverage $\theta_{\max }$, the concentration of protein $c$, the dissociation constant $K_{\mathrm{D}}$ and the Hill coefficient $H$ representing the cooperative interactions (equation 5.2).

$$
\theta=\frac{\theta_{\max }}{1+\left(\frac{K_{\mathrm{D}}}{c}\right)^{H}}
$$

Experiments of Hatzakis et al. showed that the Hill coefficient of the analyzed amphipathic helices did not change as a function of membrane curvature. ${ }^{[193]}$ The apparent dissociation constant of the peptides with the sequence of endophilin A1 decreased from $200 \pm 40 \mathrm{nM}$, measured for vesicles larger than $200 \mathrm{~nm}$, to $80 \pm 10 \mathrm{~nm}$ for $50 \mathrm{~nm}$ vesicles. In these experiments the maximum density of bound protein was dependent on the membrane curvature. As the authors observed binding of protein to highly curved structures even at saturation concentrations and as the binding affinity increased only moderately with increasing curvature they 
concluded that the density of binding sites had to increase to explain the higher amount of protein bound to highly curved vesicles. [193] Therefore, the higher apparent binding constant results from the higher binding capacity of curved membranes originating from a larger density of membrane defects and a greater exposure of the hydrophobic membrane core which has been shown to increase with increasing membrane curvature. 199-202]

The exposure of the hydrophobic bilayer core increases with increasing curvature. Membrane defects also partially expose the hydrophobic part of a lipid bilayer. POPE has a smaller head group than POPC and therefore a smaller (more negative) spontaneous curvature since the fatty acids of both phospholipids are identical. [59] The hydrophobic part of the outer monolayer of a positively curved POPC membrane containing a fraction of POPE is more exposed than that of a pure POPC membrane. This could facilitate the insertion of amphipathic helices into the lipid bilayer similar to hydrophobic defects as caused by membrane curvature and would also result in a higher apparent binding affinity.

To exclude any influences on the dissociation constant originating from the phosphoethanolamine content, SPR spectroscopy experiments with immobilized vesicles composed of POPC/PIP 2 (90:10) and POPC/POPE/PIP 2 (60:30:10) were performed. Similar dissociation constants of $K_{\mathrm{D}}\left(\mathrm{POPC} / \mathrm{PIP}_{2}\right)=(0.5 \pm 0.1) \mu \mathrm{m}$ and $K_{\mathrm{D}}\left(\mathrm{POPC} / \mathrm{POPE} / \mathrm{PIP}_{2}\right)=0.69 \pm 0.08 \mu \mathrm{m}$ were measured. This led to the conclusion that either the exposure of the hydrophobic part of the membrane is too small or not detectable within the accuracy of the experiments conducted. A smaller dissociation constant of ENTH binding to a POPC/POPE/PIP 2 membrane compared to a POPC/PIP 2 membrane would be expected as a consequence of the stronger exposure of the hydrophobic bilayer core.

Additionally to SPR spectroscopy and RIfS experiments, showing similar binding affinities, the dissociation constant was also determined by fluorescence microscopy. For this purpose, the intensity of Atto594-labeled ENTH binding to GUVs immobilized to a solid support was quantified. A dissociation constant of $0.5 \pm 0.2 \mu \mathrm{M}$ of ENTH-Atto594 binding to a DOPC/DOPE/cap-biotin-PE/Atto488DPPE/PIP 2 (66.2:30:2:1:0.8) membrane was determined by fitting equation 4.1 to the measured intensities. The dissociation constant was in the same range as measured by SPR spectroscopy for ENTH binding to a POPC/PIP 2 membrane and smaller than measured by RIfS for binding to a POPC/POPE/PIP 2 membrane. GUVs (DOPC/DOPE/cap-biotin-DOPE/Atto488-DPPE/PIP 2 , 66.2:30:2:1:0.8) ad- 
hering to an avidin-coated glass surface increase their membrane tension as a function of the $\mathrm{Mg}^{2+}$ concentration (Section 4.5.2). Membrane tension is proportional to the increase in fractional area and involves exposure of the hydrophobic part of a lipid bilayer. Therefore, the effect of high membrane tension and high membrane curvature both result in an exposure of the hydrophobic bilayer core which facilitates insertion of an amphipathic helix. The increased exposure of the hydrophobic bilayer core is schematically shown in Figure 5.1.

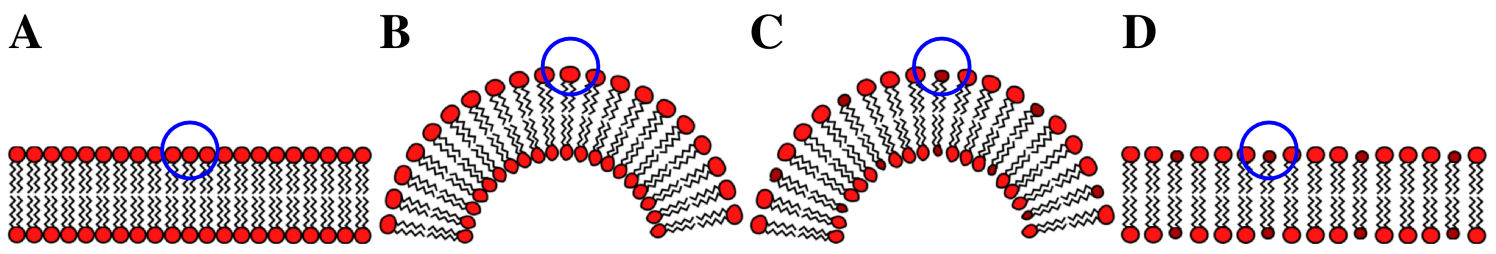

Figure 5.1: A: Schematic illustration of the exposure of the hydrophobic bilayer core of a planar membrane composed of cylindrical lipids. B: Curvature increases the exposure of the hydrophobic core. C: Presence of lipids with a small headgroup further increases the exposure of the hydrophobic core. D: Membrane tension enlarges the spacing between the lipid head groups similar to membrane curvature.

Hatzakis et al. have shown that the number of packaging defects $\left(D_{\mathrm{d}}\right)$ is proportional to membrane curvature and to the increased area $\triangle A$ of the outer bilayer leaflet of a vesicle with an area $A_{\text {vesicle }}$ (equation 5.3.

$$
D_{\mathrm{d}} \propto \frac{\Delta A}{A_{\text {vesicle }}} \propto \frac{1}{r_{\text {vesicle }}}
$$

By comparing the dissociation constants of ENTH binding to planar solidsupported membranes, to LUVs and to tensed GUVs, which have an almost planar membrane (Table 5.1), it becomes obvious that the dissociation constant obtained with GUVs is closer to the dissociation constants measured for LUVs than to planar solid-supported membranes. This could indicate a preferential binding of ENTH to tensed membranes but more experiments are needed to prove this hypothesis. It can be concluded that all dissociation constants measured in this thesis are within the range of dissociation constants measured in literature but the different values of the dissociation could not be attributed to either membrane geometry or composition. 
Electrostatic interactions have been shown to mask the ability of amphipathic helices to sense membrane curvature. ${ }^{[196]}$ ENTH's receptor lipid PIP $_{2}$ has a negative charge of -4 at $\mathrm{pH} 7$ and structural analysis of ENTH binding to inositol-1,4,5trisphosphate $(\mathrm{I}(1,4,5) \mathrm{P})$ revealed the coordination of positively charged residues of the ENTH helix to the phosphate groups of $\mathrm{I}(1,4,5) \mathrm{P}$. [46, 69] This may explain why no significantly differing apparent binding affinities were measured as the capability of ENTH to sense membrane curvature is reduced by electrostatics compared to uncharged $\alpha$-helical peptides which do not require a specific receptor lipid.

Since it has been shown that the dissociation constant of ENTH binding to $\mathrm{PIP}_{2}$ depends on the chosen experimental conditions and no dependence on the lipid composition or membrane topology was found, a mean dissociation constant of $0.6 \pm 0.1 \mu \mathrm{M}$ was calculated which is within the range of dissociation constants measured for ENTH binding to PIP $2,[13,50,51]$ The fraction of ENTH bound to the membranes used in the experiments in this thesis was estimated based on the Langmuir adsorption model (equation 4.1), the $\mathrm{PIP}_{2}$ content in the membrane and the determined mean dissociation constant of $0.6 \pm 0.1 \mu \mathrm{M}$.

\subsection{Protruded pore-spanning membranes}

\subsubsection{Curvature of protruded pore-spanning membranes}

Pore-spanning membranes have been used as a model system to analyze various processes such as SNARE-mediated membrane fusion, membrane permeabilization by antimicrobial peptides or ion single-channel recordings. [133-135] They allow to obtain a high statistic within few experiments and have a planar geometry, which mimics well the plasma membrane. Cell membranes are attached to the underlying actin-cytoskeleton which is mimicked by free-standing and solidsupported areas. However, a lot of cellular compartments are highly curved and many processes involve changes in membrane geometry. Therefore, an osmolarity gradient was applied to form protruded pore-spanning membranes with curvatures up to $1 / 425 \mathrm{~nm}^{-1}$ from the planar pore-spanning membranes suspending closed pores. ${ }^{[150]}$ These membranes with tunable curvature can be used to mimic slightly curved membranes to analyze processes which require 
membrane curvature. Immobilized vesicles are often used for experiments involving membrane curvature. 193, 203] They have a certain size distribution and are randomly localized on the surface. This is beneficial when analyzing binding as a function of membrane curvature but is a disadvantage when monitoring protein activity as a function of a specific curvature.

$\alpha$-helical peptides inserting into a membrane bilayer have shown to sense membrane curvature. The ability to sense membrane curvature is believed to decrease with increasing contribution of electrostatics. ${ }^{196]}$ The endocytotic protein epsin binds specifically to $\mathrm{PIP}_{2}$ and inserts a helix into the membrane upon binding. 47.147 It is known to induce and also to sense membrane curvature. [13, 145, 204]

To analyze not only binding but also the activity of ENTH, protruded porespanning membranes were used as a scaffold with homogeneous curvature. With the substrates used in this thesis a maximum curvature of $1 / 425 \mathrm{~nm}^{-1}$ was reached. However, the membrane curvature of various organelles is larger. The endoplasmic reticulum consists of tubules with a diameter of 30 to $50 \mathrm{~nm}$ and the cisternae located in the Golgi complex are 10 to $20 \mathrm{~nm}$ thick. [9] The size of caveolae ranges from 10 to $50 \mathrm{~nm}$ and the diameter of endocytotic vesicles from $36 \mathrm{~nm}$ to $200 \mathrm{~nm} .21,205]$ Therefore, membrane curvature relevant for processes involving the mentioned structures is in the range of $1 / 5 \mathrm{~nm}^{-1}$ to $1 / 100 \mathrm{~nm}^{-1}$.

To analyze highly curved protruded pore-spanning membranes smaller pores would be required. The size of the membrane protrusions would then deceed the diffraction limit requiring super resolution methods such as stimulated emission depletion (STED) or single-molecule localization techniques such as stochastic optical reconstruction microscopy (STORM). Those techniques involve exposure of the sample to high laser intensities (STED) resulting in bleaching of fluorophores or a statistical averaging (STORM) which decreases the time resolution. [206, 207] By using protruded pore-spanning membranes with a maximum curvature of $1 / 425 \mathrm{~nm}^{-1}$ a homogeneous scaffold with low curvature was provided to which ENTH could preferentially bind. The size of the chosen substrate allowed probing the membrane with conventional fluorescence microscopy and to analyze binding of ENTH to those membranes. 


\subsubsection{Lateral membrane tension of protruded pore-spanning membranes}

The importance of membrane tension as a regulator for different intracellular processes has become evident during the last years. ${ }^{30,208]}$ Lateral tension of lipid bilayers is used to regulate processes such as exo- and endocytosis. [15, 36, 208] Over the years a variety of methods has been developed to measure tension of cells and model membranes. Most commonly, atomic force microscopy and tether pulling using optical tweezers are used to measure membrane tension in vivo. [30] Micropipette aspiration, tether pulling by optical tweezers as well as indentation and compression by atomic force microscopy are used to measure the membrane tension of GUVs. [153, 209] Atomic force microscopy allows to locally probe the sample and was used to determine the tension of pore-spanning membranes with various pore radii, lipid compositions and in the presence or absence of organic solvent. It was shown that the lateral membrane tension is dependent on the adhesion force of the lipid bilayer to the pore rims. [119, 138, 139, 141]

Protruded pore-spanning membranes have been generated by application of an osmolarity gradient. Osmolarity gradients have been shown to stress lipid bilayers and to increase volume and surface area of cells and therewith their lateral membrane tension. Rand et al. analyzed changes in morphology and tension of red blood cells and measured an increase in membrane tension from 0.007 to $0.02 \mathrm{mN} / \mathrm{m} .{ }^{[210]}$ Membrane tension of Lymnaea neurons increased from 0.04 to $0.4 \mathrm{mN} / \mathrm{m}$ after swelling and for $\mathrm{K} 562$ cells an even larger membrane tension of $4.2 \mathrm{mN} / \mathrm{m}$ was measured after diluting the surrounding buffer and the consequent creation of hypotonic conditions. [16,211]

The lateral tension of lipid bilayers increases with increasing stretching of the lipids. Rupture of pore-spanning membranes is observed when stretching of the bilayer exceeds an area increase of 3 to $5 \%$. 141] The area of planar pore-spanning membranes with a radius of $0.425 \mu \mathrm{m}$ is $0.57 \mu \mathrm{m}^{2}$ which increases to $1.3 \mu \mathrm{m}^{2}$ if they are protruded to a spherical cap with a height equal to the pore radius. This translates into an area expansion of $100 \%$ and is impossible to reach by stretching. Since rupture of pore-spanning membranes after application of an osmolarity gradient was only rarely observed, the area increase must involve recruitment of additional lipid molecules. Fluorescence recovery after photobleaching and fluorescence loss in photobleaching (FLIP) experiments have proven the continuity 
of the lipid bilayer on the pore rim and freestanding areas. 134, 139, 212] As a conclusion, the lipid material required to compensate the increase in surface area upon protrusion of the membrane by application of an osmolarity gradient is provided by the entire membrane patch. This led to a shrinking of the patch which was imaged by fluorescence microscopy (Figure 4.7). Due to the fact that lipid material is recruited to the free-standing membrane parts, the membrane tension does not necessarily has to increase due to application of an osmolarity gradient. The lateral tension of pore-spanning membranes has been shown to depend on the adhesion of the membrane to the solid support. [141] Here, a hydrophilic functionalization of the pore rims resulted in a dramatically lower membrane tension compared to a hydrophobic functionalization. The native $\mathrm{SiO}_{2}$ layer of the silicon substrate used results in a hydrophilic surface. Since the application of an osmolarity gradient does not alter the chemical composition of the substrate, the lateral tension was expected to not change during the protrusion process. To test this hypothesis, force indentation experiments were conducted. Protruded porespanning membranes showed the same response to indentation as pore-spanning membranes on open pores. The force resulting from indentation of the protruded membranes was measured and the lateral membrane tension was calculated as for pore-spanning membranes on open pores. [119, 141, 213] The membrane tension of protruded pore-spanning membranes was determined to $2 \pm 1 \mathrm{mN} / \mathrm{m}$ by atomic force microscopy. This is higher compared to the lateral tension of cells typically ranging from 0.003 to $0.45 \mathrm{mN} / \mathrm{m}$ depending on the cell type and state. 29, 33] However, the measured membrane tension of protruded pore-spanning membranes is similar to the membrane tension of solvent free planar pore-spanning membranes and in agreement with the lateral membrane tension being determined by the adhesion of the membrane to the substrate. 119, 213]

Determining the lateral membrane tension using atomic force microscopy involves physical contact with the lipid bilayer. Another disadvantage of atomic force microscopy is its relatively low speed compared to fluorescence microscopy. Image acquisition by AFM takes typically 30 to $60 \mathrm{~s}$ depending on the image resolution and scanned area. Recent improvements have increased the speed per image to 30 to $60 \mathrm{~ms}$ for a scan range of $250 \mathrm{~nm} .{ }^{[214]}$ However, acquisition of force-distances curves, from which mechanical properties can be extracted, takes longer. Using this measurement technique, the cantilever has to approach the sample until physical contact is reached. Upon contact the cantilever further moves towards 
the sample until a previously defined force is applied. The cantilever is then retracted and approaches the next position. Hereby, the acquisition speed of an image is limited by the scanning area, the number of force-distance curves within this area (resolution), the velocity of the cantilever approaching the sample and the distance used for approaching. To avoid hydrodynamic effects which would alter the measured force, it is not possible approaching the membrane too fast. In case of the protruded pore-spanning membranes the length of the force-distance curve was determined by the expected height of the protruded pore-spanning membranes as the cantilever has to retract far enough to leave the physical contact. Another drawback of high speed atomic force microscopy compared to conventional AFM is the requirement of cantilevers with a high resonance frequency which have large spring constants. For biological samples and especially for force-distance measurements a low spring constant is beneficial. Low forces will result in a measurable detectable deflection of the cantilever which increases the measurement accuracy and allows to apply small forces. Commercially available cantilevers for high speed AFM have spring constants of $\geq 0.1 \mathrm{~N} / \mathrm{m}$ which is about a factor of 10 higher compared to the cantilevers used in this thesis. 215] Atomic force microscopy is often combined with an inverted fluorescence microscope to localize the sample and to correlate the information obtained by fluorescence microscopy and atomic force microscopy measurements. Unfortunately, the non-transparency of the silicon substrates did not allow to localize the fluorescence of pore-spanning membranes. Since localization of the protruded pore-spanning membranes using an upright microscope was not possible the substrates were replaced by $\mathrm{SiO}_{2}$ substrates and localization of membrane-sealed pores was done by inclusion of a water soluble fluorescent dye into the pores.

To overcome the limitations of AFM the lateral membrane tension of protruded pore-spanning membranes was determined using fluorescence microscopy. So far, only few methods were developed to optically determine mechanical properties of lipid bilayers. The bending rigidity, the line tension and the spontaneous curvature of GUVs were measured by flicker spectroscopy. 216, 217] Popescu et al. measured the tension coefficient of red blood cells and GUVs by stabilized Hilbert phase microscopy quantifying membrane fluctuations. [218] A FRET-based approach was used by Stabley et al. to measure mechanical forces of HCC1143 cells during early stages of endocytosis. ${ }^{[219]}$ However, measurements of lateral membrane tension using optical microscopy are rarely found. Solmaz et al. used optical stretching 
of GUVs to determine their lateral tension and bending rigidity. ${ }^{[220]}$ In this thesis, the lateral tension of protruded pore-spanning membranes was calculated from the geometry of the protruded membranes assuming a Young-Laplace behavior. Young-Laplace's law describes the relation of the pressure difference of a sphere with an interface of infinitesimal small thickness as a function of the interface tension and sphere radius (equation 4.2]. [144] Young-Laplace's law was applicable since the thickness of the lipid bilayer $(\approx 4 \mathrm{~nm})$ is much smaller than the radius of the spherical cap formed by the pore-spanning membranes after application of an osmolarity gradient. The minimal radius of $425 \mathrm{~nm}$ was reached when the height of the protruded pore-spanning membrane was equal to the pore radius. Smaller and larger membrane heights resulted in larger Laplace radii (equation 4.4). Another prerequisite for the calculation of the lateral membrane tension by fitting the pore geometry as a function of the applied osmolarity gradient is that the lateral membrane tension does not change as a function of the osmolarity gradient. Atomic force microscopy measurements did not reveal a correlation of osmolarity gradient to the lateral membrane tension. For all osmolarity gradients that were analyzed, a lateral tension in the range of 1 to $2 \mathrm{mN} / \mathrm{m}$ was measured.

The lateral membrane tension of protruded pore-spanning membranes was then calculated based on fluorescence microscopy images. Fitting of equation 4.7 to the height of the protruded pore-spanning membranes as a function of the applied osmolarity gradient yielded a membrane tension of $2 \pm 1 \mathrm{mN} / \mathrm{m}$ for protruded pore-spanning membranes on porous substrates with a pore radius of $425 \mathrm{~nm}$. For protruded pore-spanning membranes on micrometer-sized pores with a pore radius of $1.75 \mu \mathrm{m}$ a membrane tension of $2 \pm 7 \mathrm{mN} / \mathrm{m}$ was determined. The error of the membrane tension results from the uncertainty of the fitting and does not allow to obtain information about the distribution of the membrane tensions. Therefore, it was necessary to determine the membrane tension of each pore-spanning membrane individually to obtain the deviation of the lateral membrane tension of the protruded pore-spanning membranes. Fitting the height of each pore-spanning membrane as a function of the applied osmolarity gradient yielded a broad distribution of lateral tensions. For a large fraction of membranes a negative tension was determined which is physically not meaningful. Also membrane tensions larger than $10 \mathrm{mN} / \mathrm{m}$ were calculated for a significant number of membranes which is unrealistic as the lateral membrane tension of planar and protruded pore-spanning membranes determined by atomic force microscopy was 
shown to range from 1 to $3 \mathrm{mN} / \mathrm{m}$. 119, 139] Since the chemical composition of the substrate surface determining the membrane tension is not changed by application of an osmolarity gradient the membrane tension is also expected not to change. There are two main reasons for the large deviation of the lateral tensions obtained by fitting the membrane geometry applying Young-Laplace's law. Firstly, the resulting height of the protruded membranes after application of an osmolarity gradient is dependent on the geometry of the substrates (Figure 5.2). Different chemical environments lead to different adhesion and the individual pore geometries to different changes in membrane height caused by the lateral membrane tension after application of an osmolarity gradient.

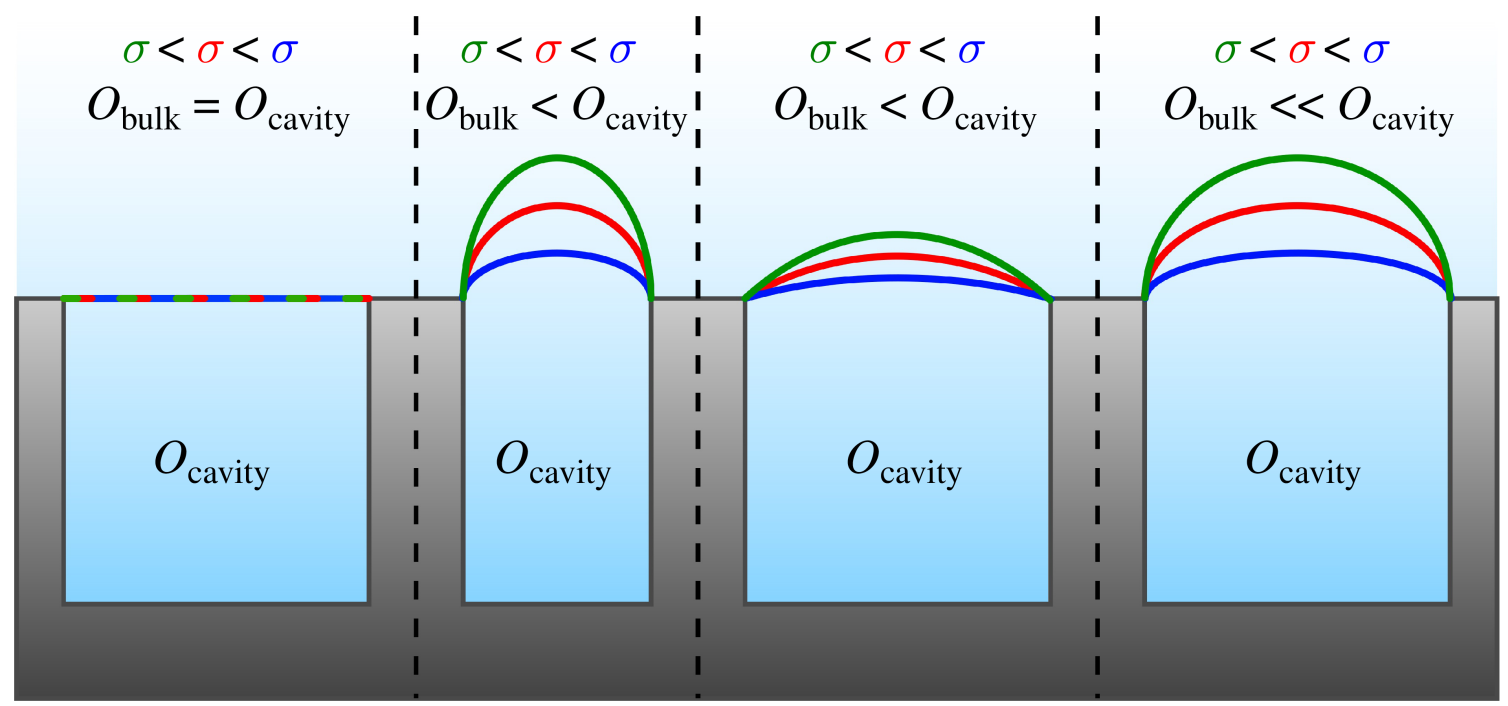

Figure 5.2: Schematic illustration of the resulting membrane height of protruded porespanning membranes after application of an osmolarity gradient for different membrane tensions and pore geometries.

As described in Section 4.4.1, the sensitivity of the tension sensor is dependent on the pore geometry, the substrate porosity, the osmolarity gradient and the osmolarity used for spreading of the GUVs. It would be advantageous to use large pore diameters to create large membrane heights since the resolution of a fluorescence microscope is limited. For a given pore geometry, the maximum osmolarity gradient before the height of the protruded PSM exceeded the pore radius increased with increasing osmolarity used for spreading of the GUVs (Figure 4.14). The number of osmolarity gradients for a given osmolarity inside the pores was then limited by the uncertainty of the osmometric measurements 
$( \pm 1 \mathrm{mOsmol} / \mathrm{L})$. Therefore, the usage of relatively high, physiologically relevant osmolarities in the range of $285 \mathrm{mOsmol} / \mathrm{L}$ is desirable as it enables to measure membrane geometries for a high number of osmolarity gradients. [221] This leads to the requirement of relatively large pore depths to ensure the desired height of the membrane. However, manufacturing pore arrays with high aspect ratios can be difficult depending on the chosen etching technique. [222-224] Therefore, the substrate available with micrometer-sized pores and the largest aspect ratio was selected and the osmolarity gradient and the osmolarity used for membrane generation were adjusted accordingly.

The second important parameter which determines the sensitivity of the tension sensor is the membrane tension itself. Pore-spanning membranes have a membrane tension of ranging from 1 to $3 \mathrm{mN} / \mathrm{m}$ and are more tensed than cellular membranes whose membrane tension ranges from 0.003 to $0.45 \mathrm{mN} / \mathrm{m}$. 29, 33, 35, 119, 139] Compared to the surface tension of water $(72.8 \mathrm{mN} / \mathrm{m})$ or organic solvents such as decane $(24.5 \mathrm{mN} / \mathrm{m})$, a solvent often used for the formation of black lipid membranes, their tension is relatively low and close to zero. 225. 226] Due to this, the decrease of the protrusion height caused by the membrane tension is relatively small compared to the theoretical case of a tension-free membrane. The simulated height of protruded pore-spanning membranes for different membrane tension is shown in Figure 5.3 A. With increasing membrane tensions and applied osmolarity gradients the difference in height of the pore-spanning membrane becomes more pronounced. For a given pore geometry and applied osmolarity gradient, the reduction in membrane height $\left(\Delta h_{\mathrm{PSM}}\right)$ increases as a function of the lateral membrane tension (Figure 5.3 B). For low membrane tensions small errors in the determination of the osmolarity or membrane height will lead to large changes in membrane tension and thereby to a broad distribution of the determined lateral tensions.

It could be shown that protruded pore-spanning membranes can be used to model not only planar membrane geometries but also as a model for curved membranes whose curvature can be tuned by the applied osmolarity gradient. In addition, they allow obtaining information about the lateral tension of the membranes which enables analysis not only of the binding of proteins and thereby the eventual changes of the membrane topology but also to analyze how the membrane tension is changed during membrane remodeling. The sensitivity of the determination of the lateral tension can be improved by manufacturing substrates with geometries 
especially optimized for the determination of lateral membrane tensions ranging from 1 to $3 \mathrm{mN} / \mathrm{m}$.
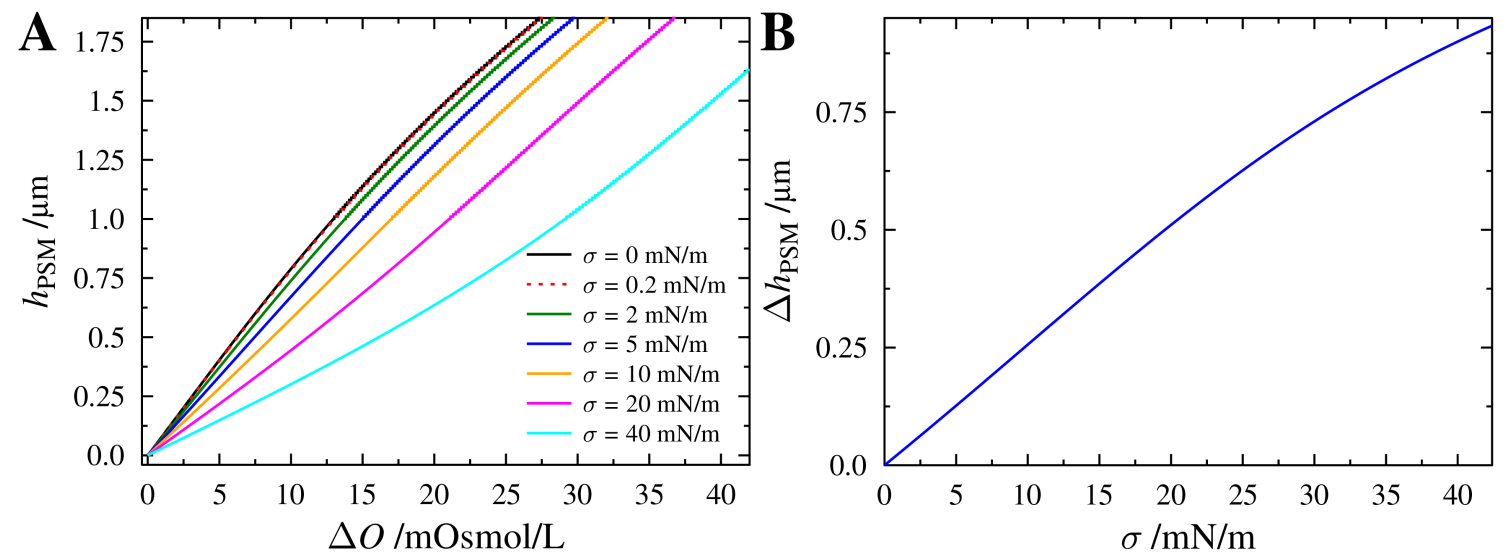

Figure 5.3: A: Heights of pore-spanning membranes as a function of the applied osmolarity gradient simulated for different membrane tensions, a pore radius of $1.75 \mu \mathrm{m}$ and an osmolarity during PSM formation of $200 \mathrm{mOsmol} / \mathrm{L}$. B: Reduction in height of protruded pore-spanning membranes as a function of the lateral membrane tension simulated for an applied osmolarity gradient of $25 \mathrm{mOsmol} / \mathrm{L}$, a pore radius of $1.75 \mu \mathrm{m}$ and an osmolarity during PSM formation of $200 \mathrm{mOsmol} / \mathrm{L}$.

\subsubsection{Interaction of ENTH with protruded pore-spanning membranes}

At equilibrium the height of protruded pore-spanning membranes after application of an osmolarity gradient is governed by their lateral membrane tension. Addition of ENTH to protruded pore-spanning membranes resulted in growth and disappearance of the membrane protrusions depending on their lipid composition (Section 4.3). In contradiction to in vivo experiments and experiments with GUVs, no vesiculation or tubulation was observed even though SDCLM measurements with a high time resolution were performed being fast enough to image an eventual vesiculation. This leads to the conclusion that binding of ENTH does not tubulate or vesiculate protruded pore-spanning membranes. Binding of ENTH to POPC (POPC/PIP $2 /$ Texas Red, 98.5:1:0.5) membranes did not result in growth but in disappearance of pore-spanning membranes (Figure $4.12 \mathrm{~B}$ ). It is conceivable that insertion of the ENTH helix into the membrane stresses the porespanning membranes resulting in pore formation and subsequent disappearance 
or rupture. Binding of ENTH to protruded pore-spanning membranes composed of DPhPC/PIP $2 /$ Texas Red (98.5:1:0.5) did not result in disappearance but in growth of the protruded membranes (Figure $4.12 \mathrm{~A}$ ). DPhPC has a high area compressibility modulus $\left(K_{A}=670 \mathrm{mN} / \mathrm{m}\right)$ and forms highly packed and ordered membranes because of its methyl-branched fatty acid chains. It is therefore mechanically stable as the intertwined network needs to be broken. 61, 62] It was used to form membranes which are expected to withstand the insertion of an amphipathic helix. POPC has a lower apparent area compressibility modulus $\left(K_{A}=213 \mathrm{mN} / \mathrm{m}\right)$ and does not form an intertwined network and is therefore mechanically less stable compared to DPhPC which might be the reason for the observed rupture of POPC membranes upon ENTH addition. [65] The hypothesis that growing of protruded pore-spanning membranes after ENTH addition prevails their disappearance for lipids forming bilayers with a higher mechanical stability is supported by experiments of protruded pore-spanning membranes containing $20 \%$ cholesterol (Figure 4.12 C). Addition of cholesterol to POPC bilayers has been shown to increase lysis tension and area compressibility modulus and therewith the stability of the membrane. 64, 65]

Growth of the protrusions after ENTH addition could be the result of either an increased surface area of the membrane protrusion or a reduced membrane tension since the membrane tension governs the protrusion height. The area of the ENTH helix was estimated to 1.6 to $4.8 \mathrm{~nm}^{2}$ representing $10-30 \%$ of the proteins footprint $\left(16 \mathrm{~nm}^{2}\right) .8$ [9] It is about two to six times larger than a $\mathrm{PIP}_{2}$ molecule $\left(\approx 0.67 \mathrm{~nm}^{2}\right) \cdot[227]$ Since $\mathrm{PIP}_{2}$ accounts only for a small fraction of the lipid bilayer the lipid areas of the matrix lipids were used (DPhPC: $0.78 \mathrm{~nm}^{2}$, POPC: $0.63 \mathrm{~nm}^{2}$ ) to approximate the change in surface area caused by insertion of the amphipathic helix. [228] Assuming a 1:1 stoichiometry of ENTH binding to $\mathrm{PIP}_{2}$, the increase in membrane area $(\triangle A)$ is then calculated as a function of the area of the ENTH helix $\left(A_{\text {helix }}\right)$, the area per lipid of the matrix lipids $\left(A_{\text {lipid }}\right)$ and the $\operatorname{PIP}_{2}\left(\chi_{\mathrm{PIP}_{2}}\right)$ content in the membrane (equation 5.4).

$$
\Delta A=\frac{A_{\text {helix }}}{A_{\text {lipid }}} \chi_{\text {PIP2 }}
$$

For a $\mathrm{PIP}_{2}$ content of $1 \mathrm{~mol} \%$ and a size of the ENTH helix of 1.6 to $4.8 \mathrm{~nm}^{2}$ this leads to an increase in membrane area of $2.4 \%$ to $7.2 \%$ for membranes with POPC as matrix lipid and of $2.1 \%$ to $6.2 \%$ for mem- 
branes with DPhPC as matrix lipid. Quantification of the protrusion radii of DPhPC (DPhPC/PIP $2 /$ Texas Red, 98.5:1:0.5) and POPC/Chol membranes (POPC/Chol/PIP $2 /$ Texas Red, 78.5:20:1:0.5) showed an increase of the mean radius of $15 \%$ for the POPC/Chol membranes and of $20 \%$ for membranes with DPhPC as the matrix lipid. To calculate the changes of the surface area a geometry of a spherical cap with a radius of the base of the cap equal to the pore radius $(0.425 \mu \mathrm{m})$ was assumed. The relative changes in surface area were then calculated based on the radii of the protruded pore-spanning membranes at the beginning of the experiment $\left(r_{0}(\mathrm{DPhPC})=0.57 \mu \mathrm{m}, r_{0}(\mathrm{POPC} / \mathrm{Chol})=0.64 \mu \mathrm{m}\right.$, Figure 4.12). The surface area of protruded DPhPC membranes increased by $54 \%$ and the surface area of the membranes composed of POPC/Chol increased by $37 \%$. Changes in osmolarity as a function of time caused by the evaporation of water decreased the radii of the protrusion as shown by the control experiments (Figure 4.12, black curves). Therefore, changes in osmolarity can be excluded as a reason for the growing protrusions. As changes in surface area cannot be attributed to changes of the osmolarity gradient and as the changes in membrane area are much larger than expected from helix insertion, a decrease of the lateral membrane tension caused by the binding of ENTH to the membranes is believed to cause the observed growth of the protrusions (Figure 5.4).

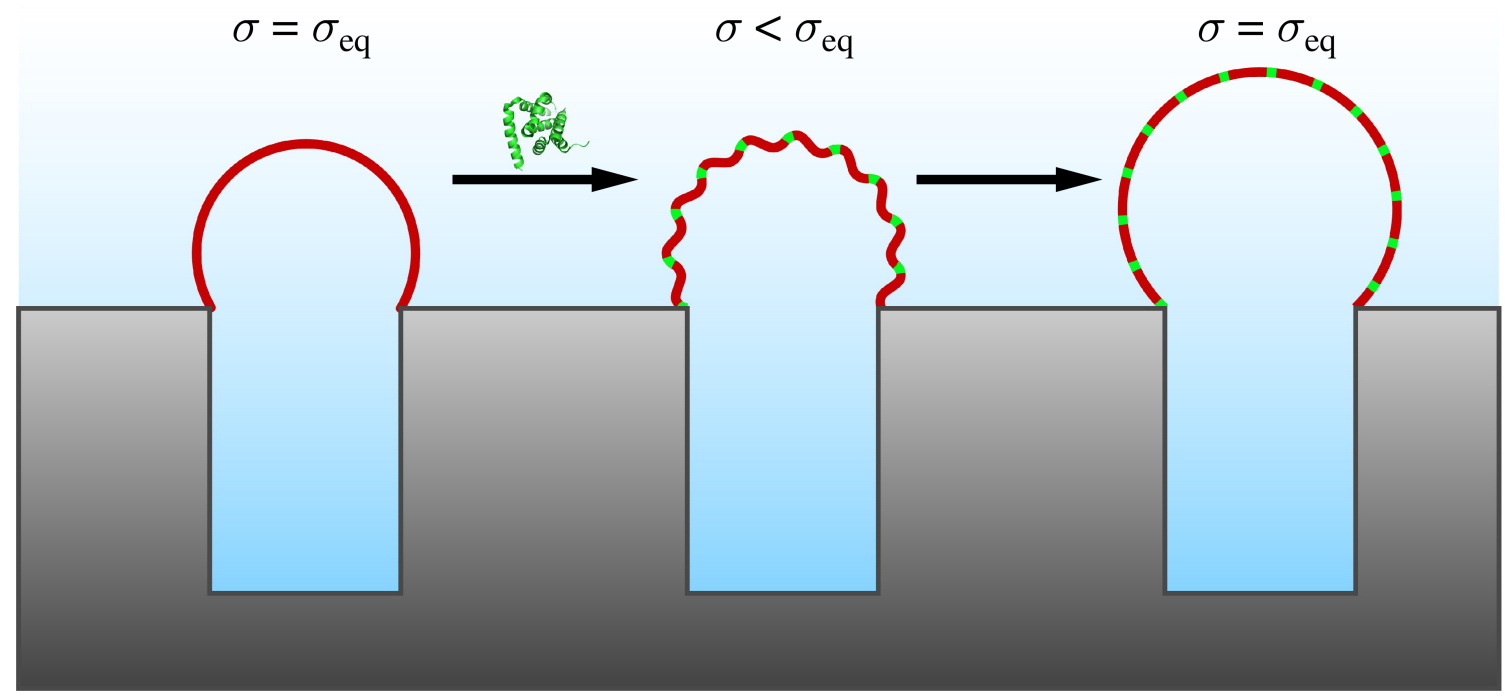

Figure 5.4: Schematic illustration of the binding of ENTH (green) decreasing the lateral tension of protruded pore-spanning membranes (red) resulting in the observed membrane growth. 
In vivo experiments have shown that exo- and endocytosis are regulated by membrane tension in addition to the complex interplay of proteins. Application of hypoosmotic conditions to $\mathrm{K} 562$ cells led to swelling of the cells and increased the membrane tension to $4.2 \mathrm{mN} / \mathrm{m}$. This led to a complete supression of endocytosis. [16] Experiments of Dai et al. support these finding showing that hypoosmotic conditions decreased the endocytotic activity of $2 \mathrm{H} 3$ cells. [15] Furthermore, a drop in membrane tension was measured when the cells were stimulated to secrete by the application of hyperosmotic conditions. Also processes such as mitosis can change membrane tension and endocytotic acitivity. Raucher and Sheetz measured that during mitosis of mammalian cells the membrane tension increased and the endocytotic acitivity was decreased. [37] When mitosis ended a decrease in membrane tension and an increased endocytotic activity was measured. The addition of amphyphilic compounds also decreased the membrane tension and increased endocytosis rate. As a conclusion the authors stated that increased membrane tension probably blocks binding of the endocytotic complex and rises the energy of membrane deformation which is required for the formation of highly curved structures such as vesicles.

The membrane tension of pore-spanning membranes was determined to 1 to $3 \mathrm{mN} / \mathrm{m}$ which is about a factor of 3-1000 larger than tensions measured in vivo. [33-35, 119, 139] Therefore, the relatively high membrane tension of the protruded pore-spanning membranes might suppress the ability of ENTH to remodel planar lipid bilayers into highly curved vesicles and membrane tubes. To analyze the binding and tubulation activity of ENTH on membranes with physiologically more relevant membrane tensions, experiments with GUVs were conducted.

\subsection{Lateral membrane tension of GUVs adhering to an avidin-coated glass surface}

\subsubsection{Adhesion of GUVs to a surface}

Membrane adhesion is involved in various biological processes such as intracellular trafficking, exocytosis and sexual reproduction. ${ }^{[171]}$ Adhesion of vesicles to surfaces has been studied extensively and has been shown to influence membrane 
tension. 229-231] Rädler and Sackmann analyzed the geometry and membranesubstrate distance of giant unilamellar vesicles on a $\mathrm{MgF}_{2}$ surface by reflectometric interference contrast microscopy (RICM). ${ }^{[170]}$ Hereby, most of the vesicles ruptured upon contact with the substrate. Strong adhesion of vesicles was then induced by addition of $10 \mathrm{mM} \mathrm{CaCl}_{2}$ to the inositol buffer resulting in strongly adhering vesicles with the shape of a spherical cap.

Ekeroth et al. analyzed the ability of divalent cations such as $\mathrm{Ca}^{2+}$ and $\mathrm{Mg}^{2+}$ to facilitate the formation of solid-supported lipid bilayers. ${ }^{\text {[232] }}$ They attributed the efficient bilayer formation to an ion specific effect mediating between membrane and substrate. This would also explain the strong adhesion of GUVs to a glass substrate in the presence of divalent cations observed by Rädler and Sackman. The influence of mono- and divalent cations on the formation of supported lipid bilayers was also studied by Seantier et al. [233] They found that the bilayer promoting effect is stronger for divalent than for monovalent cations. They measured the strongest effect for $\mathrm{Mg}^{2+}$ which was attributed to an increased vesicle-surface or vesiclevesicle adhesion.

A different strategy to induce adhesion of vesicles to a surface is the formation of biotin-avidin bonds. They are widely used to form contacts of DNA, membranes, proteins, polymers and cells. The avidin tetramer binds highly selective and with a very strong affinity to biotin $\left(K_{\mathrm{d}}=10^{-15} \mu \mathrm{M}\right) .{ }^{[75]}$ Beside the described applications, the biotin-avidin interaction has also been used to immobilize vesicles on surfaces. [166, 173] Avidin-mediated adhesion of vesicles containing biotinylated lipids was studied by micropipette aspiration as a function of the lateral membrane tension. [234] Immobilization of vesicles by formation of biotin-avidin bonds allows to induce adhesion based on a specific interaction of two binding partners and is therefore easier to control than adhesion mediated by electrostatic interactions. Both immobilization strategies, adhesion mediated by electrostatic interactions and by formation of biotin-avidin bonds, were combined to induce and control the adhesion of giant unilamellar vesicles to surfaces. $\mathrm{Mg}^{2+}$ was chosen as divalent cation to control the adhesion as it has been shown that $\mathrm{Mg}^{2+}$ facilitates bilayer formation by mediating between the membrane and the substrate and not by destabilizing the vesicles. It also had the largest promoting effect for bilayer formation. 233

The kinetics of the adhesion of vesicles to a substrate have been analyzed in great detail. 166. 235] The adhesion process is divided into three regimes with 
characteristic lengths of time. The first regime is defined by the time period the vesicle takes to reach the substrate and its actual adhesion. This process takes between $10 \mathrm{~s}$ and $1 \mathrm{~min}$ and is determined by the removal of the water layer between substrate and vesicle (Figure 5.5 I). The second regime is the adhesion of the vesicle whereby it increases its contact area. The vesicle shape becomes a spherical cap and the volume stays constant (Figure 5.5 II). Since the geometry change of the vesicle from a sphere to a spherical cap involves area dilatation, the membrane tension increases and the vesicle is under tension at the end of regime two. The increase of the contact area stops when the free energy is minimized. At equilibrium the adhesion energy resulting from the formation of biotin-avidin bonds and subsequent contact area formation is equal to the gain in elastic energy caused by the increased membrane tension. Mechanical equilibrium is reached at the end of the second regime. [171] The third regime is only observed in the case of very strong adhesion. By pore formation the vesicle leaks out and decreases its volume (Figure 5.5III).
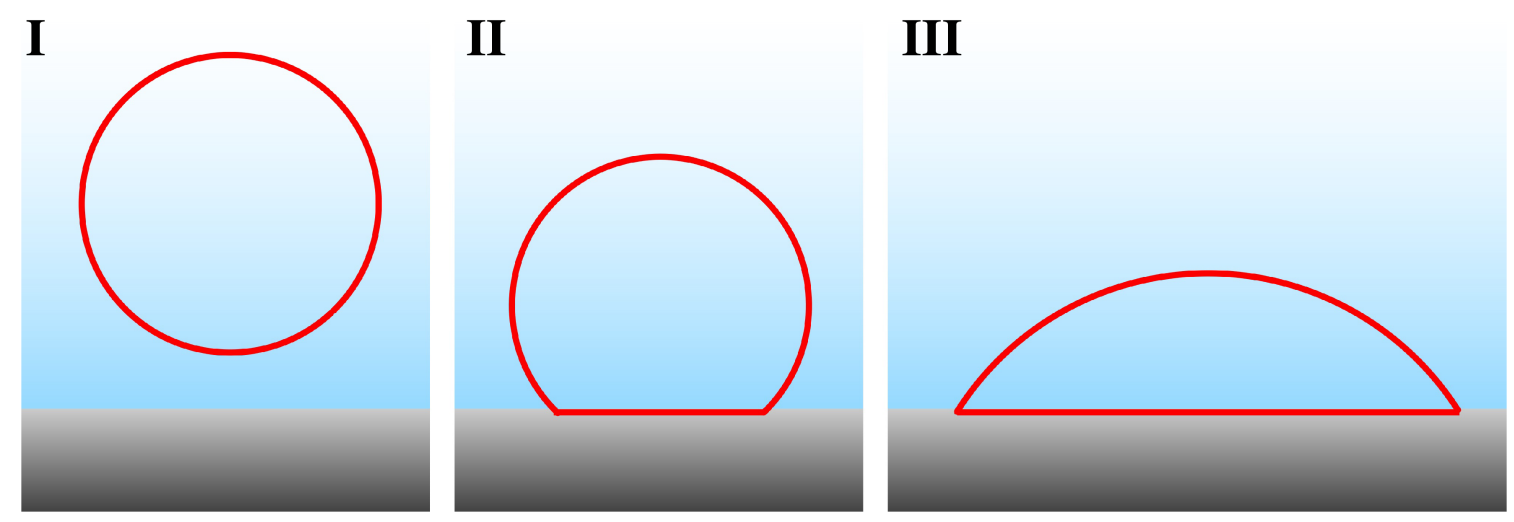

Figure 5.5: I: The vesicle approaches the substrate. II: The vesicle adheres to the substrate and forms a spherical cap. Mechanical equilibrium is reached. III: The vesicle leaks out and adopts a pancake profile. Regime III was not observed during the experiments in this thesis.

Permeabilization of water through the lipid bilayer or through the contact line caused by curvature was excluded by the authors. ${ }^{[166}$ Berthaud et al. adhered vesicles to a solid support and checked for permeation of dye molecules present in the surrounding buffer into the vesicle. They found that $93 \%$ of the vesicles did not show leakage of dye molecules. [235] Nevertheless, water molecules are much smaller than the analyzed dye molecules. This leads to the conclusion that if pores 
are formed they must be small to allow permeation of water only but not of larger molecules. Additionally, Berthaud et al. stated that no pores were formed since the size of the contact area remained constant over time similar to the results obtained in this thesis (Section 4.5.2). Pore formation can be induced by strong adhesion to the surface. The energy of pore formation decreases with increasing content of negatively charged lipids. Calculations showed that the equilibrium profile of an adhered vesicle after leakage looks like a pancake while forming a spherical cap before leakage. 231] Since the content of anionic lipids used in the experiments in this thesis was only $0.8 \%$ and all GUVs imaged had the geometry of a spherical cap and no a pancake shaped vesicles were observed, the volume of the vesicles was assumed to be constant.

Rädler et al. adhered vesicles to a substrate incubated with BSA to study and quantify membrane fluctuations using RICM. ${ }^{167}$ Passivation of the surface with BSA reduced the attractive forces between the vesicle membrane and the surface and decreased the adhesion force. Parameters such as the vesicle pre-tension, the adhesion energy to the surface and the separation distance between vesicle and substrate were measured. The pre-stress of a vesicle depends on its size and the mechanical properties of the lipid bilayer such as bending modulus and area compressibility modulus. For a vesicle in solution, the pre-tension is typically about $10^{-4} \mathrm{mN} / \mathrm{m} .{ }^{[209]}$ However, adhesion of the vesicle to a surface increases the pre-tension and the quantification of membrane fluctuations of weakly adhering vesicles to a BSA-passivated surface yielded pre-tensions of $10^{-3}$ to $10^{-1} \mathrm{mN} / \mathrm{m}$. [167]

\subsubsection{Adhesion energy and lateral membrane tension of adhered GUVs}

Thermal excitation results in membrane fluctuations and has been first described by Brochard et al. ${ }^{[160]}$ Helfrich and Servus extended the theoretical framework and performed first experiments with egg-PC vesicles. ${ }^{[161]}$ It was shown that the finite size of a vesicle leads to suppression of long wavelength fluctuations giving rise to a tension of the GUV's membrane.

Evans and Rawicz measured the lateral membrane tension of vesicles by micropipette aspiration and verified the theoretically predicted membrane tension as

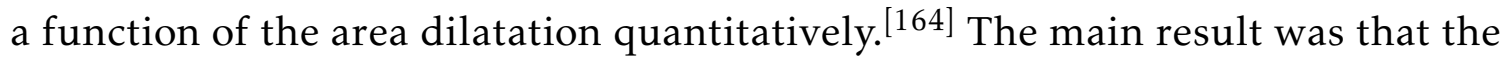


membrane tension increases very slowly upon area dilatation as long as excess area is stored in membrane fluctuations. For higher membrane tensions, when undulations are flattened and no excess area is stored in undulations, the lateral tension increases rapidly. The experimentally found relation between fractional area change $(\triangle A / A)$ and the lateral membrane tension $(\sigma)$ is given by equation 4.15 For vesicles adhering to a solid support the pre-tension was determined to be $10^{-3}$ to $10^{-1} \mathrm{mN} / \mathrm{m}$ by RICM. 167 Slightly higher pre-tensions ranging from 0.01 to $3 \mathrm{mN} / \mathrm{m}$ were measured by Schäfer et al. by compressing vesicles between two planar plates. [209]

In this thesis, the relationship between lateral membrane tension and area dilatation was used to calculate the lateral membrane tension of vesicles adhered to an avidin-coated surface. Upon adhesion the shape of a vesicle changes from a sphere to a spherical cap. Volume is conserved during this process and the surface area increases leading to an increase of the vesicle's lateral tension. The ratio of contact radius to the radius of the adhered vesicle $\left(r_{\mathrm{i}} / r_{\mathrm{ad}}\right)$ was measured as a function of the $\mathrm{Mg}^{2+}$ concentration and the corresponding tension was calculated (equation 4.23). With rising $\mathrm{Mg}^{2+}$ concentrations increasing ratios of $r_{\mathrm{i}} / r_{\mathrm{ad}}$ were measured (Section 4.5.2). When $\mathrm{PIP}_{2}$ was present in the vesicle membrane the adhesion was much stronger resulting in higher membranes tensions at identical $\mathrm{Mg}^{2+}$ concentrations compared to GUVs lacking $\mathrm{PIP}_{2}$. This is attributed to stronger interactions of the negatively charged $\mathrm{PIP}_{2}$ with the positively charged $\mathrm{Mg}^{2+}$ ions increasing the adhesion in addition to the adhesion caused by formation of biotinavidin bonds. Avidin has an iso-electric point of 10.5 and is positively charged at $\mathrm{pH} 7.4$ which further promotes the electrostatic attraction. 236] This might also increase the adhesion of a $\mathrm{PIP}_{2}$-containing membrane to the substrate. A reduction of the adhesion energy was measured in vivo when hydrolyzing $\mathrm{PIP}_{2}$ present in NIH 3T cells. 237] This indicates that PIP $_{2}$ is involved in controlling the adhesion of cells to surfaces.

In this thesis, adhesion energies were calculated according to the theoretical framework of Ramachandran et al. for adhesion in the limit of strong adhesion. [159] Then the free energy is only a function of elastic and adhesion energy and energetic contributions from membrane bending can be neglected. The strong adhesion limit can be assumed for adhesion energies larger than $1 \mu \mathrm{J} / \mathrm{m}^{2}$. 158, 175 Weak adhesion was only measured for GUVs lacking PIP 2 adhering to an avidin-coated glass surface in the presence of $1 \mathrm{~mm} \mathrm{Mg}^{2+}$. At higher $\mathrm{Mg}^{2+}$ concentrations strong 
adhesion was measured regardless if $\mathrm{PIP}_{2}$ was present or absent.

Adhesion in the strong adhesion limit was measured for different cell types. 237-239] The adhesion energy of biotinylated red blood cells adhering to a strepavidincoated surface was determined to $76 \mu \mathrm{J} / \mathrm{m}^{2}$. [240]. Moy et al. measured adhesion energies for different ligand-receptor interactions and found an adhesion energy of $200 \mu \mathrm{J} / \mathrm{m}^{2}$ for biotinylated agarose beads adhering to an avidin-coated glass surface. [241] The adhesion energies measured in these experiments are similar to the adhesion energy of $\mathrm{PIP}_{2}$-containing GUVs adhering to an avidin-coated glass surface in the presence of $0.5 \mathrm{mM} \mathrm{Mg}^{2+}$ measured in this thesis $\left(125 \mu \mathrm{J} / \mathrm{m}^{2}\right)$. Increasing adhesion energies up to $780 \mu \mathrm{J} / \mathrm{m}^{2}$ were measured as a function of the $\mathrm{Mg}^{2+}$ concentration of the surrounding buffer. To assess the adhesion energies measured at higher $\mathrm{Mg}^{2+}$ concentrations a theoretical upper limit of the adhesion energy was estimated. Assuming full coverage of the surface with avidin $\left(2 \cdot 10^{16}\right.$ avidin $\left./ \mathrm{m}^{2}\right)$ and $35 k_{\mathrm{B}} T$ per biotin-avidin bond formation a maximum adhesion energy of $2830 \mu \mathrm{J} / \mathrm{m}^{2}$ is calculated which is higher than the adhesion energies measured in this thesis. [242]

In this thesis, membrane tensions ranging from 0.01 to $1.02 \mathrm{mN} / \mathrm{m}$ were calculated based on the GUV geometry (Section 4.5.2) These tensions are lower than those of protruded pore-spanning membranes ranging from 1 to $3 \mathrm{mN} / \mathrm{m}^{[119}$ [139]. The lateral tensions of cellular membranes have been found in the range of 0.003 to $0.45 \mathrm{mN} / \mathrm{m}$ depending on the cell type and state. $29,33-35]$ The lateral tension of GUVs adhering to an avidin-coated glass surface can be tuned over almost the entire range of tensions measured in vivo. Therefore, adhered GUVs are well suited to analyze binding and activity of ENTH to lipid bilayers with physiologically relevant lateral tensions.

\subsection{Binding of ENTH to GUVs}

\subsubsection{Binding of ENTH-Atto594 to GUVs}

During the last years, the quantification of absolute fluorophore densities became more and more important for the correlation of protein number and activity. In this thesis, the quantification was performed using an external calibration standard of fluorescently labeled lipids bearing the same fluorophore as used for protein 
labeling. The intensity of GUVs doped with different amounts of the fluorescently labeled lipids was measured and plotted vs. the fluorophore content. The intensity was readout using two different evaluation strategies. For the line profile evaluation the fluorescence intensity of the membrane was quantified using a line profile in the equatorial plane of the GUV. The second evaluation was done by quantification of the intensity profile obtained in the center of the GUV in $z$-direction (axial evaluation). For both strategies the intensity was extracted as the amplitude of a Gaussian function fitted to the intensity profiles (Section 4.6.2). ${ }^{[84]}$ A prerequisite for the quantification of fluorophore surface densities was to collect the intensity from a planar membrane fragment. As GUVs have a large diameter the membrane was approximated as planar.

Different concentrations of ENTH-Atto594 were added to GUVs adhering to an avidin-coated glass surface and the intensity was quantified. Binding of ENTH-Atto594 to the GUVs was only detectable in the presence of $\mathrm{PIP}_{2}$. A binding isotherm was measured (Figure $4.30 \mathrm{C}$ ) and a dissociation constant of $0.5 \pm 0.2 \mu \mathrm{M}$ was determined by fitting the isotherm assuming a Langmuir behavior (equation 4.1). The obtained dissociation constant was comparable to those measured by RIfS and SPR spectroscopy. Using the intensity calibration and correcting for the different chemical environments of the fluorescent probe attached to either the protein or the lipid, the quantified intensities were converted into a surface density of ENTH-Atto594. The maximum surface density of ENTH-Atto594 was $0.57 \%$ of the total lipid number. This is lower than the expected $0.8 \%$ resulting from the $\mathrm{PIP}_{2}$ content assuming a 1:1 binding of ENTH to PIP 2 . Most likely the formation of $\mathrm{PIP}_{2}$ clusters is responsible for the underestimation. $\mathrm{PIP}_{2}$ molecules are known to form nanoscopic clusters in the presence of divalent cations such as $\mathrm{Mg}^{2+}$ and $\mathrm{Ca}^{2+}$. 177-[179] Even though a homogeneous distribution of $\mathrm{PIP}_{2}$ in the vesicle membrane was observed by SDCLM (Section 4.6.1), nanoscopic clusters cannot be resolved with conventional microscopy and therefore their existence can not be excluded. Since the footprint of the ENTH helix is 10 to $30 \%$ of the protein's surface projection, steric hindrance will prevent the protein to bind to $\mathrm{PIP}_{2}$ molecules in the clusters with a 1:1 stoichiometry.

Additionally to specific binding of ENTH-Atto594 to PIP ${ }_{2}$-containing GUVs the fluorescence of ENTH-Atto594 was also found at the substrate surface indicating unspecific binding of the protein. Avidin has an isoelectric point (pI) of 10.5 and is therefore positively charged at $\mathrm{pH}$ 7.4. [236] The $\mathrm{pI}$ of ENTH was computed to 
8.0 based on the protein's sequence using the ProtParam tool. 48, 70] ENTH has 22 negatively charged residues (aspartic acid and glutamic acid) and 23 positively charged residues (arginine and lysine). Therefore, it is also positively charged at $\mathrm{pH} 7.4$ even though the net charge is expected to be smaller compared to avidin since the $\mathrm{pI}$ is closer to the $\mathrm{pH}$. Interactions of ENTH with the also positively charged avidin will be hampered by repulsive interactions. However, labeling of ENTH with Atto594 introduced an additional negative charge to the protein. [72] This may be sufficient to promote interactions of ENTH and avidin since the isoelectric point of ENTH is only slightly above the $\mathrm{pH}$ used for the experiments. Protein activity and stability of ENTH-Atto594 were altered by the attached fluorophore. Addition of ENTH-Atto594 to GUVs led neither to tubulation nor to flattening of the vesicles. Most likely, flattening is prevented due to ENTHAtto594 molecules sticking to the avidin molecules blocking the binding pockets of avidin for biotin. It is only possible to speculate about the cause for the absence of tubulation or vesiculation. Protein crowding is discussed to contribute to the generation of membrane curvature. ${ }^{[8]}$ Thus, it is conceivable that the fluorophore increases the steric hindrance of the proteins reducing the protein concentration on the surface below a certain level required for the formation of tubules and vesicles. As tubulation was only rarely observed when using non-labeled ENTH, its absence could be the result of a too low number of vesicles analyzed. Furthermore, UV/VIS measurements have shown that the attached fluorophore decreases the protein's stability. Precipitation of labeled protein was observed and leads to a systematic misevaluation of the protein surface densities. To measure the non-altered activity of ENTH binding to artificial membranes and to avoid precipitation of protein further experiments were performed with non-labeled ENTH.

\subsubsection{Quantification of the changes in membrane area caused by ENTH binding}

Binding of ENTH to GUVs led to flattening of the adhered vesicles increasing the ratio of $r_{\mathrm{i}} / r_{\mathrm{ad}}$ regardless of the vesicle's lateral tension (Section 4.7.2). Changes of the ratio of $\Delta\left(r_{\mathrm{i}} / r_{\mathrm{ad}}\right)$ of $0.07,0.08$ and 0.07 were measured for $\mathrm{Mg}^{2+}$ concentrations of $0.5 \mathrm{~mm}, 1 \mathrm{~mm}$ and $2 \mathrm{~mm}$, respectively. The increase in vesicle area caused by the space of the ENTH helix was estimated to $1.2 \%$ to $3.6 \%$ based on the $\mathrm{PIP}_{2}$ content in the membrane, the determined dissociation constant, the ENTH concentration 
and the crystal structure of the protein. As the observed change in membrane area was $78 \pm 9 \%$, assuming a constant compressibility modulus before and after ENTH binding of $265 \mathrm{mN} / \mathrm{m}$, the area increase caused by the inserted ENTH helix was too low explaining it. Therefore, the area compressibility modulus had to decrease and an apparent area compressibility modulus of $149 \pm 9 \mathrm{mN} / \mathrm{m}$ was determined (Section 4.7.2 neglecting the small changes in area caused by insertion of the ENTH helix. As the bending rigidity is linearly related to the area compressibility modulus (equation 4.31), a reduction of the area compressibility modulus translates into a reduction of the bending rigidity.

Softening of membranes was also observed after binding of the $\alpha$-helical peptide magainin-2, the HIV-1 peptide and the protein SAR1 which is involved in vesicle trafficking. 189, 190, 243] Magainin-2 led to softening of POPC membranes and was measured by fluctuation analysis. ${ }^{[189]}$ A reduction of the bending rigidity from $24 k_{\mathrm{B}} T$ to $5.6 k_{\mathrm{B}} T$ was measured with the softening being a function of the peptide fraction on the membrane. The softening was attributed to the formation of local regions with high curvature. For the HIV-1 peptide a similar reduction of the bending rigidity of DOPC membrane from $20 k_{\mathrm{B}} T$ to $6.5 k_{\mathrm{B}} T$ was measured by x-ray diffraction. [190] Micropipette aspiration and fluctuation anaylsis yielded bending rigidities of 10 to $16 k_{\mathrm{B}} T$ after HIV-1 addition to DOPC membranes. [243] Even though the peptide was added to both sites of the bilayer, the authors concluded that the reduction in bending rigidity might involve the formation of spontaneous curvature. A reduction of the membrane bending rigidity is also caused by the endocytotic protein SAR1 and was measured by tether pulling using optical tweezers. [191] Similar to ENTH, SAR1 forms an amphipathic helix at the $\mathrm{N}$-terminal end of the protein which inserts into a membrane bilayer being the first step in the formation of a vesicle at the endoplasmic reticulum. SAR1 is able to tubulate vesicles with a lipid composition resembling those of the ER. Similar to ENTH, its tubulation activity was also dependent on the presence of a helix at the $\mathrm{N}$-terminal part of the protein. The reduction in bending rigidity by SAR1 from $13 k_{\mathrm{B}} T$ to $5 k_{\mathrm{B}} T$ was measured as a function of the solution concentration of the peptide.

The reduction in bending rigidity by ENTH measured in this thesis is similar to the reduction measured for other proteins. The absolute value of the bending rigidity is strongly dependent on the buffer composition, especially on the sugar and salt content. [243,244] The relative reduction of the bending rigidity after binding of 
helical peptides measured by different groups using inherent different techniques ranges from a factor of 1.3 to 4.3 and is comparable with the results obtained in this thesis $\left(\kappa_{\mathrm{DOPC}} / \kappa_{\mathrm{DOPC}, \mathrm{ENTH}}=1.8\right)$. Micropipette aspiration experiments of GUVs analyzing binding of ENTH to highly curved and tensed membrane tethers revealed a preferential binding of ENTH to the highly curved membrane regions. ENTH preferentially partitioned into membrane tubes with high membrane tension and curvature and relocated to the planar vesicles membrane if the tension was lowered. 245] According to Leibler's curvature coupling model the elastic energy per unit area of a fluid continuous bidimensional membrane is given by:

$$
F=\frac{1}{2} \kappa\left(C-C_{\mathrm{s}}\right)^{2}
$$

with $\kappa$ being the bending modulus, $C$ the mean curvature of the membrane ( $\left.C=1 / r_{1}+1 / r_{2}\right)$ and $C_{\mathrm{s}}$ being the spontaneous curvature accounting for membrane asymmetry. [246] The bending rigidity is reduced by insertion of particles due to preference of the particle for a specific curvature. Then effective bending rigidity $\kappa_{\text {eff }}$ is given by:

$$
\kappa_{\mathrm{eff}}=\kappa-\Lambda^{2} \cdot \chi
$$

with $\chi$ being the osmotic compressibility and $\Lambda$ being the coupling constant. The bending rigidity is decreased as a result of the curvature preference of the protein. The reduction is a function of the density of proteins and $\Lambda$ describing the coupling strength. Rearrangement of equation 5.6 approximating $\chi$ as an ideal gas yields:

$$
\Lambda=\sqrt{\frac{\left(\kappa-\kappa_{\mathrm{eff}}\right) \rho k_{\mathrm{B}} T}{x_{\mathrm{PIP} 2}}},
$$

with $\rho$ being the lipid density $\left(\rho=a_{1}^{-1}\right)$ and $x_{\mathrm{PIP} 2}$ the mole fraction of $\mathrm{PIP}_{2}$ bound to ENTH. For the calculation of the coupling constant a density of the ENTH-PIP $2^{-}$ complex of $x_{\mathrm{PIP} 2}=0.5 \%$ of the total lipids was calculated based on the Langmuir adsorption model using the determined dissociation constant of $K_{\mathrm{D}}=0.6 \mu \mathrm{M}$ and a concentration of ENTH of $1 \mu \mathrm{M}$ as used in the experiments. By further using an area per lipid of $a_{\mathrm{DOPC}}=0.7 \mathrm{~nm}^{2}$, a bending modulus of $\kappa=21 k_{\mathrm{B}} T$ before ENTH addition and an effective bending modulus of $\kappa_{\text {eff }}=12.5 \pm 0.6 k_{\mathrm{B}} T$ after ENTH binding, a coupling constant of $\Lambda=-201 \pm 6 \mathrm{pN}$ was calculated using equation 5.7 which is close to the value measured by Capraro et al. $(\Lambda=-146 \pm 3 \mathrm{pN})$. [245] 
On a molecular scale, insertion of a peptide or amphipathic helix can lead to a membrane thinning decreasing the mechanically relevant hydrophobic thickness and therewith the bending rigidity (Figure 5.6 A). Depending on the penetration depth, a helix can also reduce the coupling between the two membrane monolayers $(\beta)$ which lowers the bending rigidity (Figure 5.6 B, equation 4.31). Also a general weakening of the bilayer by altered lipid packing is conceivable. To visualize a reduction of the apparent area compressibility modulus as a result of the insertion of an amphipathic helix, one might think of the helix as a foreign body which disturbs the hydrophobic interactions and therewith lowers the energy required for stretching of the bilayer (Figure $5.6 \mathrm{C}$ ). On a molecular scale this means that a constant force, e.g. applied to the membrane by an osmotic gradient, is able to further increase the area of the membrane protrusion as the interactions withstanding the area enlargement are lowered.

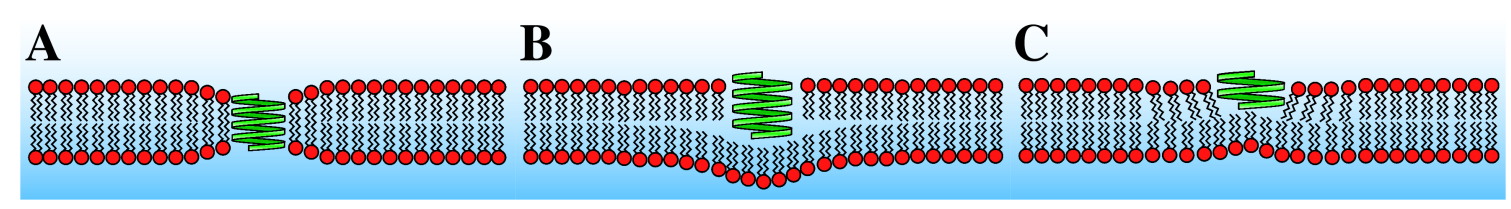

Figure 5.6: A: Membrane thinning as a result of helix insertion. B: Enlarged spacing of the lipid monolayers as a result of helix insertion. C: Disordered lipid packing as a result of helix insertion.

\subsubsection{Binding of ENTH to GUVs with a low membrane tension}

ENTH has been shown to cause tubulation of non-adhered GUVs, even though no tubulation or vesiculation of protruded pore-spanning membranes was observed after treatment with ENTH $(c=1 \mu \mathrm{M}) .8$, 87 , 145] The membrane tension of GUVs is originating from thermal fluctuations and is typically in the range of $10^{-4} \mathrm{mN} / \mathrm{m}$ for freely floating GUVs. ${ }^{209}$ This tension is significantly lower than the membrane tension of $0.08 \mathrm{mN} / \mathrm{m}$ of GUVs adhering to an avidin-coated glass surface at $0.5 \mathrm{~mm}$ $\mathrm{Mg}^{2+}$ (Section 4.5.2). However, after ENTH addition to adhered vesicles with a lateral tension of $0.08 \mathrm{mN} / \mathrm{m}$ the formation of tubular structures was observed for a small fraction of the GUVs. The formation of highly curved structures is caused by the insertion of the ENTH helix into the lipid bilayer which generates an asymmetry between the two membrane monolayers (Figure 5.7). 44, 47] 


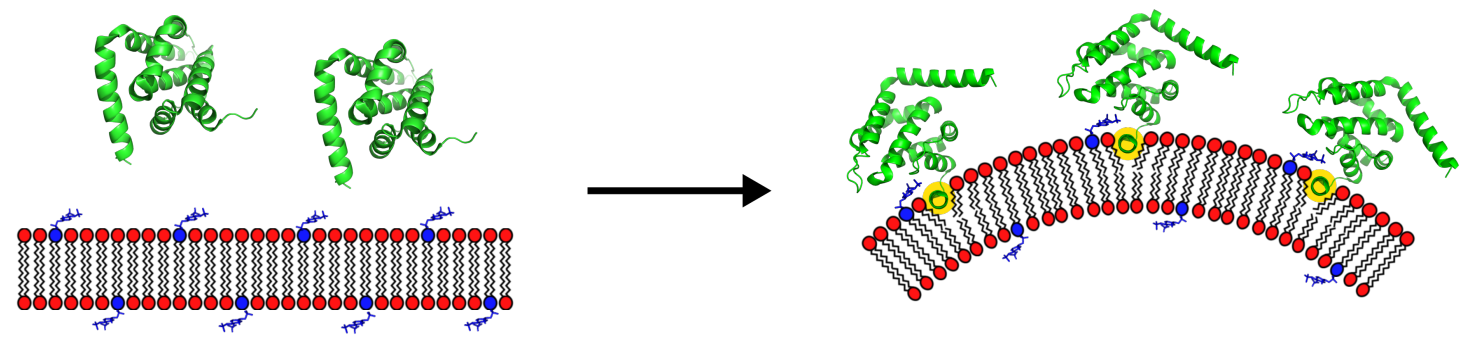

Figure 5.7: Schematic illustration of ENTH (green) binding to a lipid bilayer (red) containing $\mathrm{PIP}_{2}$ (blue). Helix insertion (yellow) splays the lipid head groups generating an asymmetry between the monolayers leading to membrane curvature.

The tubulation of only a fraction of the vesicles is attributed to the higher membrane tension of the adhered vesicles compared to the freely floating GUVs and to kinetics aspects of the tubulation process. Therefore, binding of ENTH to adhered GUVs can either result in the formation of membrane tubes or flattening of the vesicles (Figure 5.8).

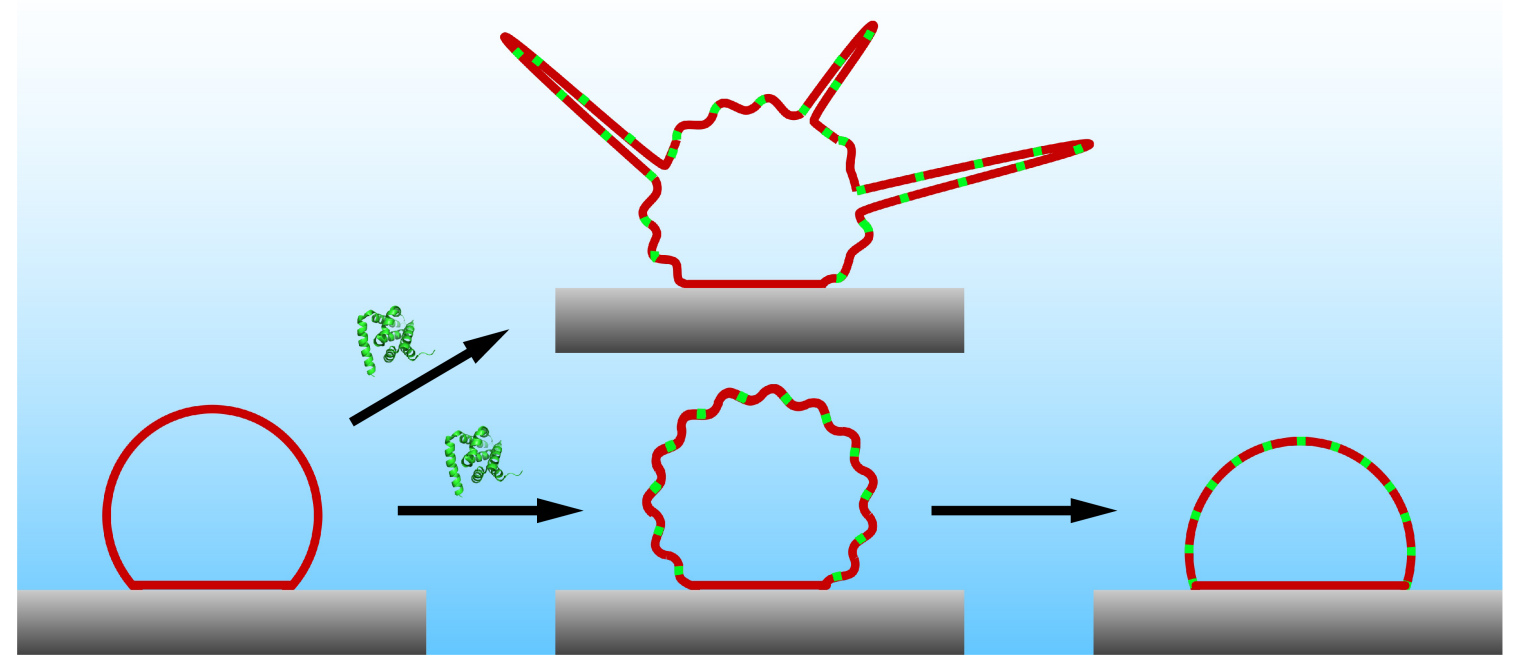

Figure 5.8: Schematic illustration of ENTH (green) binding to adhered GUVs (red) resulting in either formation of membrane tubes or flattening of the vesicle.

Boucrot et al. proposed a theoretical model which predicts the formation of vesicles or tubes based on the protein to lipid ratio and the ratio of the scaffold $\kappa_{\mathrm{p}}$ to the lipid bending modulus $\kappa_{\mathrm{m}} \cdot{ }^{[52]}$ According to the model, the formation of membrane tubes requires a lower surface density of proteins of a given $\kappa_{\mathrm{p}} / \kappa_{\mathrm{m}}$. The model 
was used to predict the formation of tubular and vesicular structures caused by scaffolding proteins bearing amphipathic helices such as the endocytotic protein N-BAR. Proteins such as ENTH which do not have scaffolding properties and induce membrane curvature only by insertion of an amphipathic helix are predicted to form vesicular structures independent of the protein to lipid ratio. However, the formation of membrane tubes after ENTH addition has been observed in various studies. 44, 47] Therefore, the model is not able to fully predict the type of structures caused by ENTH binding. In agreement with the theoretical model of Boucrot et al. simulations by Lai et al. have shown that the formation of membrane tubules as a result of ENTH binding is favored at a low protein density on the membrane surface leading to growth of ENTH aggregates forming a protein lattice on the membrane tubules. If the ENTH concentration is initially very high, vesicles are preferentially formed. [148]

For the $\mathrm{PIP}_{2}$ content $(0.8 \%)$ of the GUVs used for the experiments in this thesis, the ENTH density was calculated using equation 5.4 assuming a 1:1 binding of ENTH and $\mathrm{PIP}_{2}$. To calculate the surface coverage of protein and not the increase in area caused by the ENTH helix the area of the helix was replaced by the protein's footprint $\left(16 \mathrm{~nm}^{2}\right)$ and an area per lipid of $A_{\mathrm{DOPC}}=0.7 \mathrm{~nm}^{2}$ was used. ${ }^{247}$ At saturation concentrations this translates into a maximum surface coverage with ENTH of $18.3 \%$. Taking into account the mean dissociation constant of $0.6 \mu \mathrm{M}$ for ENTH binding to $\mathrm{PIP}_{2}$ and assuming a Langmuir behavior the coverage drops to $11.4 \%$ for the used ENTH concentration of $1 \mu \mathrm{M}$. This relatively low coverage explains the predominant formation of tubular structures. Additionally, the formation of a vesicle which detaches is more complex compared to the formation of a membrane tube making it unfavorable. 186

Generation of highly curved structures can also be caused by protein crowding. A high protein density at the membrane induces local curvature which bends the membrane. Experiments with ENTH lacking the helical part responsible for inserting into the membrane support this mechanism. However, protein surface densities of $20 \%$ and more were necessary to generate membrane curvatures resulting in tubule formation. ${ }^{[8]}$ Due to the low receptor lipid content of $0.8 \%$ and the used concentration of ENTH of $1 \mu \mathrm{M}$ the expected surface coverage of $11.4 \%$ is below the $20 \%$ coverage required to induce tubulation by protein crowding. Therefore, protein crowding is expected to have, if any, only a minor contribution in generating membrane curvature. 
It is concluded that a low membrane tension decreases the energy barrier for remodeling of a planar membrane into a highly curved one and that the predominant formation of tubular structures originates from the low surface coverage with protein resulting from the chosen experimental conditions. The reduction of the bending rigidity due to the insertion of the ENTH helix is believed being a major contribution in the generation of membrane curvature. The reduction of the bending stiffness caused by ENTH binding lowers the elastic energy per unit area (equation 5.5). Hence, the energy required to deform a flat membrane into a highly curved vesicle or membrane tether is decreased. In combination with the generation of local spontaneous curvature due to the inserted helix this may efficiently promote the observed tubulation of the vesicles with a low membrane tension after binding of ENTH.

\subsubsection{Theoretical predictions for membrane curvature inducing proteins}

Additionally to experimental approaches, the formation of tubular structures has been modeled theoretically. Tourdot et al. calculated the free energy landscape of curvature-inducing proteins on membrane bilayers. ${ }^{[248]}$ They analyzed the ability of proteins, approximated as points inducing spontaneous curvature, to bend membranes for different parameters such as protein concentration on the surface and membrane area which is inversely proportional to the membrane tension. Simulations revealed an increasing area of a fluctuating membrane $\left(A_{\mathrm{fl}}\right)$ compared to the area of its projection $\left(A_{\mathrm{p}}\right)$ as a function of the number of proteins for a given spontaneous curvature. For a constant protein number, an increase in $A_{\mathrm{fl}} / A_{\mathrm{p}}$ was found as a function of the spontaneous curvature. Furthermore, the authors also showed that the presence of membrane undulations decreases the effective bending rigidity and softens the membrane which lowers the energy required for membrane deformation.

Later, Tourdot et al. extended their model to analyze more parameters and to compare the theoretical predictions with experimental results. The formation of tubular structures was modeled as a function of the protein number, the spontaneous curvature and $A_{\mathrm{fl}} / A_{\mathrm{p}}$. The number of membrane tubules increased with increasing protein field density. More proteins inducing smaller spontaneous curvatures were required for the formation of membrane tubes compared to proteins inducing 
a large spontaneous curvature similar to previous calculations. No tubes were formed at low values of $A_{\mathrm{fl}} / A_{\mathrm{p}}$ which corresponds to a high membrane tension. Tubes also did not form if the spontaneous curvature was too low which then only leads to the formation of membrane blebs.

To assess the theoretical predictions, the authors compared their model with micropipette experiments. N-BAR, an endocytotic protein which also inserts a helix into a membrane monolayer, was used for comparison. The critical effective membrane tension, defined as the membrane tension at which tube formation started to become inhibited, was calculated as a function of protein surface density for different spontaneous curvatures corresponding to different radii of membrane tubes. Small radii required a lower surface concentration of proteins with large spontaneous curvature while large radii and thus smaller curvature fields required a larger protein density. Importantly, higher membrane tensions required a larger protein density to result in formation of membrane tubules. For a given membrane tension, tubulation can therefore be induced by either increasing the protein density at constant membrane tension or lowering the membrane tension at constant protein density. [249] In this thesis, for GUVs with a membrane tension of $0.08 \mathrm{mN} / \mathrm{m}$ tubulation was observed after binding of ENTH. However, not all vesicles formed tethers. Based on the theoretical considerations, the membrane tension of the vesicles was still above the critical tension to result in tube formation or the protein density was too low. Increasing the $\mathrm{PIP}_{2}$ concentration would allow obtaining higher protein densities and thereby facilitate tubulation.

Interestingly, Tourdot et al. found an increased membrane tension with increasing protein number as the protein density also alters the membrane fluctuations. An increase in membrane tension of biotinylated GUVs adhered to an avidincoated glass surface would cause a decrease of the vesicles' contact area which requires dissociation of biotin-avidin bonds. The biotin-avidin bonds are extremely stable when formed once. The biotin-avidin complex is stable in a $\mathrm{pH}$ range of 2 to 13.7 and in $9 \mathrm{M}$ urea. The thermal stability of the complex is increased compared to individual proteins and has a very low dissociation rate constant of $7.5 \cdot 10^{-8} \mathrm{~s}^{-1}$.250,251] Therefore, dissociation of the biotin-avidin complex is unlikely. Additionally, the simulated rise of the membrane tension upon protein insertion is only $0.02 \mathrm{mN} / \mathrm{m}$ for the largest protein number simulated. 249] An increase of the lateral membrane tension of GUVs adhering to an avidin-coated glass surface at $\mathrm{Mg}^{2+}$ concentration of $0.5 \mathrm{~mm}$ would lead to a decrease of $r_{\mathrm{i}} / r_{\mathrm{ad}}$ of 
-0.023 assuming a pre-tension of $10^{-2} \mathrm{mN} / \mathrm{m}$ (Figure 4.22). Assuming a contact area of $2 \mu \mathrm{m}$, the reduction of the contact area diameter is smaller than $133 \mathrm{~nm}$ (1 pixel) and could barely be resolved. For higher $\mathrm{Mg}^{2+}$ concentrations and higher membrane tensions the decrease of $r_{\mathrm{i}} / r_{\text {ad }}$ would be even smaller. Additionally, fluctuations in vesicles with high membrane tension are flattened and undulations are suppressed for membrane tensions larger than $0.5 \mathrm{mN} / \mathrm{m}$. 169] Then, insertion of proteins into an undulation-free membrane should only increase the membrane area which corresponds to a decrease of the lateral tension as area and tension are inversely related. The insertion of the ENTH helix also reduces the vesicles' area compressibility modulus which causes the observed flattening of the vesicles.

\subsubsection{Binding of ENTH to GUVs with high membrane tension}

Binding of ENTH to GUVs with a mean membrane tension of $0.59 \mathrm{mN} / \mathrm{m}$ only led to flattening of the GUVs increasing their contact area whereas the formation of membrane tubes was not observed. As the radius did not significantly change, the ratio of $r_{\mathrm{i}} / r_{\mathrm{ad}}$ increased due to ENTH binding (Section 4.7.1). The size of the contact area of an adhered vesicle is determined by an equilibrium between adhesion force and membrane tension. [171] The free energy is reduced by the formation of biotin-avidin bonds which is compensated by the increasing lateral membrane tension. Flattening of the vesicles after ENTH binding is a consequence of the reduced bending rigidity (Section 5.4.2). A lower bending stiffness translates into a decreased area compressibility modulus (equation 4.31). As the size of the contact area is determined by the equilibrium of adhesion energy and elastic energy (equation 4.9) and as ENTH binding to the vesicle does not alter the adhesion energy per unit area, a reduction in $K_{A}$ results in an enlargement of the vesicle's area. Area dilatation and membrane tension are proportional with the area compressibility modulus being the proportional factor (equation 4.13). Therefore, the vesicle flattens and enlarges its contact area until the vesicle's lateral membrane tension reaches the same value as before protein binding which is defined by the mechanical equilibrium.

Similar to experiments with protruded pore-spanning membranes, no vesiculation or tubulation was observed after ENTH addition. [150] Most likely, the high membrane tension prevents the formation of highly curved structures such as vesicles or tubules as a high membrane tension increases the energy required for 
membrane deformation. 252.253] Asymmetric insertion of an amphipathic helix into a membrane has been shown to generate spontaneous membrane curvature which induces membrane tension. The increased tension would further rise the energy required for membrane deformation and thus prevents the formation of highly curved structures. [254] The suppression of the endocytotic activity as measured for cells, whose membrane tension was increased, e.g. by external conditions such as osmolarity gradients, supports this hypothesis. [16, 211, 219] Formation of a vesicle during clathrin mediated endocytosis takes about 10 to $20 \mathrm{~s}$ and involves recruitment of several proteins. ${ }^{[255]}$ From this time scale it might be hypothesized that the formation of vesicles or tubules is slower than the flattening process. Otherwise, a fast local decrease in membrane tension would allow the formation of highly curved structures.

It has been shown that strong adhesion is achieved for biotin concentrations larger than $0.5 \mathrm{~mol} \%$. 256] A typical GUV with $2 \mathrm{~mol} \%$ of cap-biotin-DOPE and a radius of $20 \mu \mathrm{m}$ forms an adhesion area of $438 \mu \mathrm{m}^{2}\left(r_{\mathrm{i}} / r_{\mathrm{ad}}=0.59\right)$. This translates into $6 \%$ of the cap-biotin-DOPE molecules being located in the contact region, assuming full coverage with avidin $\left(2 \cdot 10^{-16} \mathrm{~m}^{2}\right) \cdot{ }^{[241]}$ Thus, non-bound biotinylated lipids exist being in proximity to the avidin-coated surface. Therefore, vesicle flattening only requires small changes in vesicle geometry to result in an energetically favored bond formation, whereas the formation of vesicles and tubules involves the reorganization of large membrane parts. However, the recently discovered pathway of ultra fast endocytosis is much faster than clathrin mediated endocytosis and does require mechanisms which allow to initiate membrane budding and vesicle formation on a millisecond time scale. ${ }^{[28]}$ Changes in membrane tension could be used to rapidly decrease the energy required for membrane deformation and could initiate rearrangement of the lipid bilayer.

Another explanation that high membrane tension inhibits the formation of highly curved structures has been provided by Simunovic et al. [257] The authors simulated the aggregation of N-BAR, a protein also involved in endocytosis, as a function of membrane tension. Simulations revealed that N-BAR forms linear aggregates and induces membrane curvature. The aggregation of N-BAR was prevented by high membrane tension and thereby the formation of membrane tubes with high curvature was inhibited. The linear N-BAR aggregates were required for the formation of membrane tubules and were almost completely suppressed at a membrane tension of $0.25 \mathrm{mN} / \mathrm{m}$. Yoon et al. discovered the formation of 
ENTH oligomers by number and brightness analysis of ENTH bound to GUVs. 47] Aggregates with 10 to 100 ENTH molecules were observed after 4 min and the experiments showed that tubulation started after formation of aggregates of 20-22 ENTH molecules. Therefore, the inhibited protein aggregation caused by high membrane tension might contribute to the suppression of membrane tubules.

Recently, the function of the actin-cytoskeleton in clathrin mediated endocytosis was analyzed. Actin polymerization was confirmed to provide the energy required for clathrin mediated endocytosis if the force from the endocytotic proteins was insufficient. [42, 258] The force necessary to deform a flat lipid bilayer into a bent vesicle rises with increasing membrane tension. ${ }^{[253]}$ Recent in vivo experiments support the hypothesis of high membrane tension suppressing the formation of vesicles by ENTH as they have shown that cells use a previously unknown binding site at the C-terminus of epsin for binding to actin. It is believed that binding of epsin to actin is used to overcome the force originating from membrane tension which suppresses membrane invaginations. [43]

Summarizing the results obtained in this thesis, the formation of highly curved vesicles and membrane tubules is a result of ENTH changing the mechanical properties of lipid bilayers and external factors such as membrane tension preventing or facilitating their formation. A high membrane tension efficiently inhibits the generation of membrane curvature whereas lowering the lateral membrane tension can facilitate their formation by reducing the elastic energy.

The insertion of the ENTH helix disturbs the hydrophobic interactions of the lipids altering the mechanical properties of the membrane. Depending on the parameter of interest this leads to a reduction of the bending rigidity, the area compressibility modulus or the membrane tension. The observed growth of protruded porespanning membranes, which was attributed to a reduction in membrane tension, can also be attributed to a decreased area compressibility modulus (equation 4.13). Both, the observed growth of pore-spanning membranes and flattening of GUVs adhering to an avidin-coated surface are different observations originating from the identical mode of action of ENTH. 


\section{Conclusion}

During endocytosis, a flat membrane buds and forms a vesicle which then detaches and transports the enclosed cargo. A complex protein machinery is required to overcome the energy barrier for membrane remodeling and to induce the necessary changes in membrane topology. During the last years it became evident that celluar processes are also regulated by physical properties of the involved membranes.

In this thesis, binding of the epsin N-terminal homology domain (ENTH) was analyzed using artificial lipid bilayers to study how the membrane remodeling ability of ENTH changes as a function of membrane tension and lipid composition. Furthermore, it was investigated how physical properties of the membrane are altered by binding of ENTH.

Protruded pore-spanning membranes were established to analyze the activity of ENTH as a function of the lipid composition. For this purpose, pore-spanning membranes on closed pores were protruded by the application of an osmolarity gradient. The height of the membrane protrusions is governed by the lateral membrane tension and was quantified as a function of the applied osmolarity gradient. A mean membrane tension of $2 \mathrm{mN} / \mathrm{m}$ was determined and validated by indentation experiments.

To analyze the remodeling activity of ENTH as a function of different lateral membrane tensions, experiments with biotinylated giant unilamellar vesicles (GUVs) adhered to an avidin-coated glass surface were conducted. The lateral tension was calculated from the vesicles' geometry and tuned by adjusting the adhesion strength of the vesicles using buffers with different concentrations of $\mathrm{Mg}^{2+}$. This allowed investigating binding of ENTH to lipid bilayers with lateral tensions ranging from 0.08 to $0.59 \mathrm{mN} / \mathrm{m}$. 
To analyze the binding of ENTH to $\mathrm{PIP}_{2}$ as a function of lipid comoposition and topology, binding isotherms were measured by reflectometric interference spectroscopy, surface plasmon spectroscopy and quantitative fluorescence microscopy. Similar affinities in the high nanomolar range were measured regardless of the analyzed lipid composition and topology.

The activity of ENTH was then analyzed as a function of lipid composition using protruded pore-spanning membranes. Binding of ENTH to the protruded pore-spanning membranes resulted in growth, flattening and rupture of the membrane protrusions. As the height of the pore-spanning membranes is governed by the lateral tension, the increase in height was attributed to a reduction in tension. Flattening and subsequent rupture was attributed to a breakdown of the osmolarity gradient as a result of the formation of membrane defects. Lipid bilayers having a high lysis tension and area compressibility modulus such as DPhPC are mechanically stable and could withstand the insertion of the ENTH helix, thus mainly growth of the membranes was observed. Binding of ENTH to mechanically less stable membranes such as POPC resulted in rupture of the protruded porespanning membranes. No vesiculation and tubulation was observed after binding of ENTH to protruded pore-spanning membranes. To examine whether ENTH's remodeling ability is suppressed by a high membrane tension, experiments with GUVs sessile adhered were conducted.

No tubulation and vesiculation was detected after binding of ENTH to GUVs with a membrane tension of $0.59 \mathrm{mN} / \mathrm{m}$. GUVs with a lower membrane tension of $0.08 \mathrm{mN} / \mathrm{m}$ were tubulated by ENTH, showing that the formation of highly curved membrane structures is dependent on the lateral tension. For all tensions analyzed, flattening of the vesicles was observed after binding of ENTH. This was attributed to a reduction of the membrane's area compressibility modulus caused by the insertion of a helix of ENTH, which disturbs the packing of the lipids in the membrane. The decreased area compressibility modulus lowers the energy required for membrane deformation and is the molecular explanation of the observed growth of the protruded pore-spanning membrane after binding of ENTH. In combination with the generation of membrane curvature by splaying the lipid head groups, insertion of amphipathic helices can efficiently initiate the formation of a vesicle. 


\section{Bibliography}

[1] S. Sigismund, S. Confalonieri, A. Ciliberto, S. Polo, G. Scita, P. P. Di Fiore, Endocytosis and signaling: Cell logistics shape the eukaryotic cell plan, Physiol. Rev. 2012, 92(1), 273-366.

[2] M. M. Kozlov, H. T. McMahon, L. V. Chernomordik, Protein-driven membrane stresses in fusion and fission, Trends Biochem. Sci. 2010, 35(12), 699 706.

[3] H. T. McMahon, J. L. Gallop, Membrane curvature and mechanisms of dynamic cell membrane remodelling, Nature 2005, 438(7068), 590-596.

[4] H. T. McMahon, E. Boucrot, Membrane curvature at a glance, J. Cell Sci. 2015, 128(6), 1065-1070.

[5] S. Daum, D. Krüger, A. Meister, J. Auerswald, S. Prinz, J. A. G. Briggs, $\mathrm{K}$. Bacia, Insights from reconstitution reactions of COPII vesicle formation using pure components and low mechanical perturbation, Biol. Chem. 2014, 395(7-8), 801-812.

[6] H. Lè Ne Boudin, A. Doan, J. Xia, R. Shigemoto, R. L. Huganir, P. Worley, A. M. Craig, Presynaptic clustering of mGluR7a requires the PICK1 PDZ domain binding site, Neuron 2000, 28(2), 485-497.

[7] S. A. Eckler, R. Kuehn, M. Gautam, Deletion of N-terminal rapsyn domains disrupts clustering and has dominant negative effects on clustering of fulllength rapsyn, Neuroscience 2005, 131(3), 661-670.

[8] J. C. Stachowiak, E. M. Schmid, C. J. Ryan, H. S. Ann, D. Y. Sasaki, M. B. Sherman, P. L. Geissler, D. A. Fletcher, C. C. Hayden, Membrane bending by protein-protein crowding, Nat. Cell Biol. 2012, 14(9), 944-949. 
[9] M. M. Kozlov, F. Campelo, N. Liska, L. V. Chernomordik, S. J. Marrink, H. T. McMahon, Mechanisms shaping cell membranes, Curr. Opin. Cell Biol. 2014, $29,53-60$.

[10] H. T. McMahon, I. G. Mills, COP and clathrin-coated vesicle budding: Different pathways, common approaches, Curr. Opin. Cell Biol. 2004, 16(4), 379-391.

[11] K. Bacia, E. Futai, S. Prinz, A. Meister, S. Daum, D. Glatte, J. A. G. Briggs, R. Schekman, Multibudded tubules formed by COPII on artificial liposomes, Sci. Rep. 2011, 1, 17.

[12] A. Frost, V. M. Unger, P. De Camilli, The BAR domain superfamily: Membrane-molding macromolecules, Cell 2009, 137(2), 191-196.

[13] M. G. J. Ford, I. G. Mills, B. J. Peter, Y. Vallis, G. J. K. Praefcke, P. R. Evans, H. T. McMahon, Curvature of clathrin-coated pits driven by epsin, Nature 2002, 419(6905), 361-366.

[14] G. J. Doherty, H. T. McMahon, Mediation, modulation, and consequences of membrane-cytoskeleton interactions, Annu. Rev. Biophys. 2008, 37(1), 65-95.

[15] J. Dai, H. P. Ting-Beall, M. P. Sheetz, The secretion-coupled endocytosis correlates with membrane tension changes in RBL 2H3 cells, J. Gen. Physiol. 1997, 110(1), 1-10.

[16] C. Rauch, E. Farge, Endocytosis switch controlled by transmembrane osmotic pressure and phospholipid number asymmetry, Biophys. J. 2000, 78(6), 30363047.

[17] S. I. Bannykh, T. Rowe, W. E. Balch, The organization of endoplasmic reticulum export complexes, J. Cell Biol. 1996, 135(1), 19-35.

[18] B. Antonny, Membrane deformation by protein coats, Curr. Opin. Cell Biol. 2006, 18(4), 386-394.

[19] T. Mitsunari, F. Nakatsu, N. Shioda, P. E. Love, A. Grinberg, J. S. Bonifacino, H. Ohno, Clathrin adaptor AP-2 is essential for early embryonal development, Mol. Cell. Biol. 2005, 25(21), 9318-9323. 
[20] H. Chen, G. Ko, A. Zatti, G. Di Giacomo, L. Liu, E. Raiteri, E. Perucco, C. Collesi, W. Min, C. Zeiss, P. De Camilli, O. Cremona, Embryonic arrest at midgestation and disruption of Notch signaling produced by the absence of both epsin 1 and epsin 2 in mice, Proc. Natl. Acad. Sci. U.S.A. 2009, 106(33), 13838-13843.

[21] H. T. McMahon, E. Boucrot, Molecular mechanism and physiological functions of clathrin-mediated endocytosis, Nat. Rev. Mol. Cell Biol. 2011, 12(8), 517-533.

[22] G. J. Doherty, H. T. McMahon, Mechanisms of endocytosis, Annu. Rev. Biochem. 2009, 78, 857-902.

[23] G. J. K. Praefcke, H. T. McMahon, The dynamin superfamily: Universal membrane tubulation and fission molecules?, Nat. Rev. Mol. Cell Biol. 2004, 5(2), 133-147.

[24] M. Meinecke, E. Boucrot, G. Camdere, W. C. Hon, R. Mittal, H. T. McMahon, Cooperative recruitment of dynamin and BIN/Amphiphysin/Rvs (BAR) domain-containing proteins leads to GTP-dependent membrane scission, J. Biol. Chem. 2013, 288(9), 6651-6661.

[25] R. Nossal, Energetics of clathrin basket assembly, Traffic 2001, 2(2), 138-147.

[26] A. G. Clark, O. Wartlick, G. Salbreux, E. K. Paluch, Stresses at the cell surface during animal cell morphogenesis, Curr. Biol. 2014, 24(10), R484-R494.

[27] S. Watanabe, B. R. Rost, M. Camacho-Pérez, M. W. Davis, B. Söhl-Kielczynski, C. Rosenmund, E. M. Jorgensen, Ultrafast endocytosis at mouse hippocampal synapses, Nature 2013, 504(7479), 242-247.

[28] Z. Shi, T. Baumgart, Membrane tension and peripheral protein density mediate membrane shape transitions, Nat. Commun. 2015, 6(5974), 1-8.

[29] A. D. Lieber, S. Yehudai-Resheff, E. L. Barnhart, J. A. Theriot, K. Keren, Membrane tension in rapidly moving cells is determined by cytoskeletal forces, Curr. Biol. 2013, 23(15), 1409-1417.

[30] A. Diz-Munoz, D. A. Fletcher, O. D. Weiner, Use the force: Membrane tension as an organizer of cell shape and motility, Trends Cell Biol. 2013, 23(2), 47-53. 
[31] T. A. Masters, B. Pontes, V. Viasnoff, Y. Li, N. C. Gauthier, Plasma membrane tension orchestrates membrane trafficking, cytoskeletal remodeling, and biochemical signaling during phagocytosis, Proc. Natl. Acad. Sci. U.S.A. 2013, 110(29), 11875-1880.

[32] M. M. Kozlov, L. V. Chernomordik, Membrane tension and membrane fusion, Curr. Opin. Struct. Biol. 2015, 33, 61-67.

[33] C. E. Morris, U. Homann, Cell surface area regulation and membrane tension, J. Membrane Biol. 2001, 179(2), 79-102.

[34] Y. Schweitzer, A. D. Lieber, K. Keren, M. M. Kozlov, Theoretical analysis of membrane tension in moving cells, Biophys. J. 2014, 106(1), 84-92.

[35] A. Pietuch, B. B. R. Brückner, T. Fine, I. Mey, A. Janshoff, Elastic properties of cells in the context of confluent cell monolayers: Impact of tension and surface area regulation, Soft Matter 2013, 9(48), 11490-11502.

[36] N. C. Gauthier, M. A. Fardin, P. Roca-Cusachs, M. P. Sheetz, Temporary increase in plasma membrane tension coordinates the activation of exocytosis and contraction during cell spreading, Proc. Natl. Acad. Sci. U.S.A. 2011, 108(35), 14467-14472.

[37] D. Raucher, M. P. Sheetz, Membrane expansion increases endocytosis rate during mitosis, J. Cell Biol. 1999, 144(3), 497-506.

[38] J. Liu, Y. Sun, G. F. Oster, D. G. Drubin, Mechanochemical crosstalk during endocytic vesicle formation, Curr. Opin. Cell Biol. 2010, 22(1), 36-43.

[39] D. R. Kovar, T. D. Pollard, Insertional assembly of actin filament barbed ends in association with formins produces piconewton forces, Proc. Natl. Acad. Sci. U.S.A. 2004, 101(41), 14725-14730.

[40] J. L. Salisbury, J. S. Condeelis, P. Satir, Role of coated vesicles, microfilaments, and calmodulin in receptor-mediated endocytosis by cultured B lymphoblastoid cells, J. Cell Biol. 1980, 87(1), 132-141.

[41] M. Kaksonen, C. P. Toret, D. G. Drubin, Harnessing actin dynamics for clathrin-mediated endocytosis, Nat. Rev. Mol. Cell Biol. 2006, 7(6), 404-414. 
[42] S. Boulant, C. Kural, J.-C. Zeeh, F. Ubelmann, T. Kirchhausen, Actin dynamics counteract membrane tension during clathrin-mediated endocytosis, Nat. Cell Biol. 2011, 13(9), 1124-1131.

[43] M. Skruzny, T. Brach, R. Ciuffa, S. Rybina, M. Wachsmuth, M. Kaksonen, Molecular basis for coupling the plasma membrane to the actin cytoskeleton during clathrin-mediated endocytosis, Proc. Natl. Acad. Sci. U.S.A. 2012, 109(38), E2533-E2542.

[44] C. A. Horvath, D. Vanden Broeck, G. A. Boulet, J. Bogers, M. J. De Wolf, Epsin: Inducing membrane curvature, Int. J. Biochem. Cell Biol. 2007, 39(10), 1765-1770.

[45] V. Legendre-Guillemin, S. Wasiak, N. K. Hussain, A. Angers, P. S. McPherson, ENTH/ANTH proteins and clathrin-mediated membrane budding, J. Cell Sci. 2004, 117(1), 9-18.

[46] T. Itoh, P. De Camilli, BAR, F-BAR (EFC) and ENTH/ANTH domains in the regulation of membrane-cytosol interfaces and membrane curvature, $B B A$ Mol. Cell Biol. L. 2006, 1761(8), 897-912.

[47] Y. Yoon, J. Tong, P. J. Lee, A. Albanese, N. Bhardwaj, M. Källberg, M. A. Digman, H. Lu, E. Gratton, Y. K. Shin, W. Cho, Molecular basis of the potent membrane-remodeling activity of the epsin 1 N-terminal homology domain, J. Biol. Chem. 2010, 285(1), 531-540.

[48] D. Fremont, PDB ID 1EYH, Crystal structure of the epsin N-terminal homology domain (ENTH) domain at 1.56 Angstrom resolution, 2000, http: //www.rcsb.org/pdb/explore/explore.do?structureId=1EYH, accessed: Mar. 2016.

[49] M. Ford, I. Mills, B. Peter, Y. Vallis, G. Praefcke, P. Evans, H. McMahon, PDB ID 1H0A, Epsin bound to Ins(1,4,5)P3, 2002, http://www.rcsb.org/pdb/ explore/ explore.do?structureId=1H0A, accessed: May 2016.

[50] R. V. Stahelin, F. Long, B. J. Peter, D. Murray, P. De Camilli, H. T. McMahon, W. Cho, Contrasting membrane interaction mechanisms of AP180 N-terminal homology $(\mathrm{ANTH})$ and epsin N-terminal homology (ENTH) domains, J. Biol. Chem. 2003, 278(31), 28993-28999. 
[51] R. A. Hom, M. Vora, M. Regner, O. M. Subach, W. Cho, V. V. Verkhusha, R. V. Stahelin, T. G. Kutateladze, pH-dependent binding of the epsin ENTH domain and the AP180 ANTH domain to PI(4,5)P2-containing bilayers, J. Mol. Biol. 2007, 373(2), 412-423.

[52] E. Boucrot, A. Pick, G. Çamdere, N. Liska, E. Evergren, H. T. McMahon, M. M. Kozlov, Membrane fission is promoted by insertion of amphipathic helices and is restricted by crescent BAR domains, Cell 2012, 149(1), 124-136.

[53] O. Cremona, P. De Camilli, Phosphoinositides in membrane traffic at the synapse, J. Cell Sci. 2001, 114(6), 1041-1052.

[54] D. Harries, J. Rösgen, A practical guide on how osmolytes modulate macromolecular properties, Method. Cell Biol. 2008, 84(7), 679-735.

[55] W. J. Hamer, Y.-C. Wu, Osmotic coefficients and mean activity coefficients of univalent electrolytes in water at $25^{\circ} \mathrm{C}$, J. Phys. Chem. Ref. Data 1972, 1(4), 1047-1100.

[56] K. Miyajima, M. Sawada, M. Nakagaki, Studies on aqueous solutions of saccharides. I. Activity coefficients of monosaccharides in aqueous solutions at $25^{\circ} \mathrm{C}$, B. Chem. Soc. Jpn. 1983, 56(6), 1620-1623.

[57] T. E. Sweeney, C. A. Beuchat, Limitations of methods of osmometry: Measuring the osmolality of biological fluids, Am. J. Physiol. 1993, 264(3), R469R480.

[58] G. van Meer, D. R. Voelker, G. W. Feigenson, Membrane lipids: Where they are and how they behave, Nat. Rev. Mol. Cell Biol. 2008, 9(2), 112-124.

[59] B. Kollmitzer, P. Heftberger, M. Rappolt, G. Pabst, Monolayer spontaneous curvature of raft-forming membrane lipids, Soft Matter 2013, 9(45), 1087710884 .

[60] W. Shinoda, M. Mikami, T. Baba, M. Hato, Molecular dynamics study on the effect of chain branching on the physical properties of lipid bilayers: Structural stability, J. Phys. Chem. B 2003, 107(50), 14030-14035.

[61] W. Shinoda, K. Shinoda, T. Baba, M. Mikami, Molecular dynamics study of bipolar tetraether lipid membranes, Biophys. J. 2005, 89(5), 3195-3202. 
[62] I. van Uitert, S. Le Gac, A. van den Berg, The influence of different membrane components on the electrical stability of bilayer lipid membranes, $B B A$ Biomembranes 2010, 1798(1), 21-31.

[63] P. W. Bruce Alberts, Alexander Johnson, Julian Lewis, Martin Raff, Keith Roberts, Molecular biology of the cell, 5th edition, Taylor \& Francis Ltd, 2007, 622-624.

[64] D. Needham, R. S. Nunn, Elastic deformation and failure of lipid bilayer membranes containing cholesterol, Biophys. J. 1990, 58(4), 997-1009.

[65] J. Henriksen, A. C. Rowat, E. Brief, Y. W. Hsueh, J. L. Thewalt, M. J. Zuckermann, J. H. Ipsen, Universal behavior of membranes with sterols, Biophys. J. 2006, 90(5), 1639-1649.

[66] W. Wang, L. Yang, H. W. Huang, Evidence of cholesterol accumulated in high curvature regions: Implication to the curvature elastic energy for lipid mixtures, Biophys. J. 2007, 92(8), 2819-2830.

[67] T. F. Martin, PI(4,5)P2 regulation of surface membrane traffic, Curr. Opin. Cell Biol. 2001, 13(4), 493-499.

[68] G. Di Paolo, P. De Camilli, Phosphoinositides in cell regulation and membrane dynamics, Nature 2006, 443(7112), 651-657.

[69] S. McLaughlin, D. Murray, Plasma membrane phosphoinositide organization by protein electrostatics, Nature 2005, 438(7068), 605-611.

[70] E. Gasteiger, C. Hoogland, A. Gattiker, S. Duvaud, M. R. Wilkins, R. D. Appel, A. Bairoch, The Proteomics Protocols Handbook, 1st edition, Humana Press, Totowa, NJ, 2005, 571-607.

[71] Invitrogen, Alexa Fluor ${ }^{\circledR} 488$ protein labeling kit, 2006, https: // tools thermofisher.com/content/sfs/manuals/mp10235.pdf, accessed: May 2016.

[72] ATTO-Tec, Recommended procedures for labeling proteins with aminereactive ATTO-labels, 2014, https://www.atto-tec.com/fileadmin/ user_upload/Katalog_Flyer_Support/Procedures.pdf, accessed: Jan. 2016. 
[73] P. Gyorgy, C. S. Rose, R. B. Eakin, E. E. Snell, R. J. Williams, Egg-white injury as the result of nonabsorption or inactivation of biotin, Science 1941, 93, $477-478$.

[74] M. Wilchek, E. A. Bayer, O. Livnah, Essentials of biorecognition: The (strept)avidin-biotin system as a model for protein-protein and proteinligand interaction, Immunol. Lett. 2006, 103(1), 27-32.

[75] E. P. Diamandis, T. K. Christopoulos, The biotin-(strept)avidin system: Principles and applications in biotechnology, Clin. Chem. 1991, 37(5), 625636.

[76] J. W. Lichtman, J.-A. Conchello, Fluorescence microscopy, Nat. Methods 2005, 2(4), 910-919.

[77] M. Born, E. Wolf, Principles of optics, 7th edition, Cambridge University Press, Campbridge, 2003, 436-445.

[78] H. Kirshner, D. Sage, M. Unser, 3D PSF models for fluorescence microscopy in ImageJ, Proceedings of the Twelfth International Conference on Methods and Applications of Fluorescence Spectroscopy, Imaging and Probes (MAF'11), $2011,154$.

[79] H. Kirshner, F. Aguet, D. Sage, M. Unser, 3-D PSF fitting for fluorescence microscopy: Implementation and localization application, J. Microsc. 2013, 249(1), 13-25.

[80] R. W. Cole, T. Jinadasa, C. M. Brown, Measuring and interpreting point spread functions to determine confocal microscope resolution and ensure quality control, Nat. Protoc. 2011, 6(12), 1929-1941.

[81] E. H. K. Stelzer, Contrast, resolution, pixelation, dynamic range and signalto-noise ratio: Fundamental limits to resolution in fluorescence light microscopy, J. Microsc. 1998, 189(1), 15-24.

[82] J. B. Pawley, Handbook of biological confocal microscopy, 3rd edition, Springer Science+Business Media, New York, 2006.

[83] R. Gräf, J. Rietdorf, T. Zimmermann, Live cell spinning disk microscopy, Adv. Biochem. Eng. Biot. 2005, 95, 57-75. 
[84] J.-A. Conchello, J. W. Lichtman, Optical sectioning microscopy, Nat. Methods 2005, 2(12), 920-931.

[85] S. Wilhelm, B. Gröbler, M. Gluch, H. Heinz, Confocal laser scanning microscopy, http: //zeiss-campus .magnet.fsu.edu/referencelibrary/ pdfs/ZeissConfocalPrinciples.pdf, accessed: Apr. 2016.

[86] D. K. Toomre, M. F. Langhorst, M. W. Davidson, Introduction to spinning disk microscopy, http://zeiss-campus.magnet.fsu.edu/articles/ spinningdisk/introduction.html, accessed: May 2016.

[87] E. Wang, C. M. Babbey, K. W. Dunn, Performance comparison between the high-speed Yokogawa spinning disc confocal system and single-point scanning confocal systems, J. Microsc. 2005, 218(2), 148-159.

[88] T. Shimozawa, K. Yamagata, T. Kondo, S. Hayashi, A. Shitamukai, D. Konno, F. Matsuzaki, J. Takayama, S. Onami, H. Nakayama, Y. Kosugi, T. M. Watanabe, K. Fujita, Y. Mimori-Kiyosue, Improving spinning disk confocal microscopy by preventing pinhole cross-talk for intravital imaging, Proc. Natl. Acad. Sci. U.S.A. 2013, 110(9), 3399-3404.

[89] M. Hirsch, R. J. Wareham, M. L. Martin-Fernandez, M. P. Hobson, D. J. Rolfe, A stochastic model for electron multiplication charge-coupled devices - from theory to practice, PLoS One 2013, 8(1), e53671.

[90] M. S. Robbins, S. Member, B. J. Hadwen, The noise performance of electron multiplying charge-coupled devices, IEEE T. Electron Dev. 2003, 50(5), $1227-$ 1232.

[91] Andor Technology Ltd., Specifications Andor iXon 897 ultra, http://www.andor.com/pdfs/specifications/Andor_iXon_Ultra_ 897_Specifications.pdf, accessed: Apr. 2016.

[92] Andor Technology Ltd., Specification Andor Neo 5.5, http: / / www . andor com/pdfs/specifications/Andor_Neo_sCMOS_Specifications.pdf, accessed: Apr. 2016.

[93] H. T. Beier, B. L. Ibey, Experimental comparison of the high-speed imaging performance of an EM-CCD and sCMOS camera in a dynamic live-cell imaging test case, PLoS One 2014, 9(1), e84614. 
[94] F. K. Chong, C. G. Coates, D. J. Denvir, N. G. McHale, K. D. Thornbury, M. A. Hollywood, Optimization of spinning disk confocal microscopy: Synchronization with the ultra-sensitive EMCCD, Proc. SPIE 5324, Three-Dimensional and Multidimensional Microscopy: Image Acquisition and Processing XI, 2004, $65-76$.

[95] P. Walde, K. Cosentino, H. Engel, P. Stano, Giant vesicles: Preparations and applications, ChemBioChem. 2010, 11(7), 848-865.

[96] M. I. Angelova, D. S. Dimitrov, Liposome electroformation, Faraday Discuss. Chem. Soc. 1986, 81, 303-311.

[97] X. Fan, I. M. White, S. I. Shopova, H. Zhu, J. D. Suter, Y. Sun, Sensitive optical biosensors for unlabeled targets: A review, Anal. Chim. Acta 2008, 620(1), $8-26$.

[98] J. Homola, Surface Plasmon Resonance Based Sensors, Springer Berlin Heidelberg New York, 2006, 25-32.

[99] W. Knoll, Interfaces and thin films as seen by bound electromagnetic waves, Annu. Rev. Phys. Chem. 1998, 49(1), 569-638.

[100] G. Gauglitz, Direct optical sensors: Principles and selected applications, Anal. Bioanal. Chem. 2005, 381(1), 141-155.

[101] G. Gauglitz, A. Brecht, G. Kraus, W. Nahm, Chemical and biochemical sensors based on interferometry at thin (multi-)layers, Sens. Actuators B Chem. 1993, 11(1), 21-27.

[102] G. E. Jellison, Optical functions of GaAs, GaP, and Ge determined by twochannel polarization modulation ellipsometry, Opt. Mater. 1992, 1(3), 151160.

[103] I. H. Malitson, Interspecimen comparison of the refractive index of fused silica, J. Opt. Soc. Am. 1965, 55(10), 1205.

[104] P. Schiebener, J. Straub, J. M. H. Levelt Sengers, J. S. Gallagher, Refractive index of water and steam as function of wavelength, temperature and density, J. Phys. Chem. Ref. Data 1990, 19(3), 677-717. 
[105] J. A. Braunger, C. Kramer, D. Morick, C. Steinem, Solid supported membranes doped with PIP2: Influence of ionic strength and $\mathrm{pH}$ on bilayer formation and membrane organization, Langmuir 2013, 29(46), 1420414213.

[106] K. Tawa, K. Morigaki, Substrate-supported phospholipid membranes studied by surface plasmon resonance and surface plasmon fluorescence spectroscopy, Biophys. J. 2005, 89(4), 2750-2758.

[107] J. Vörös, The density and refractive index of adsorbing protein layers, Biophys. J. 2004, 87(1), 553-561.

[108] G. Binnig, H. Rohrer, C. Gerber, E. Weibel, Tunneling through a controllable vacuum gap, Appl. Phys. Lett. 1982, 40(2), 178-180.

[109] G. Binnig, C. F. Quate, Atomic force microscope, Phys. Rev. Lett. 1986, 56(9), 930-933.

[110] E.-L. Florin, V. T. Moy, H. E. Gaub, Adhesion forces between individual ligand-receptor pairs, Science 1994, 264(5157), 415-417.

[111] M. Rief, M. Gautel, F. Oesterhelt, J. M. Fernandez, H. E. Gaub, Reversible unfolding of individual titin immunoglobulin domains by AFM, Science 1997, 276(5315), 1109-1112.

[112] K. K. C. Neuman, A. Nagy, Single-molecule force spectroscopy: Optical tweezers, magnetic tweezers and atomic force microscopy, Nat. Methods 2008, 5(6), 491-505.

[113] I. Mey, C. Steinem, A. Janshoff, Biomimetic functionalization of porous substrates: Towards model systems for cellular membranes, J. Mater. Chem. 2012, 22(37), 19348-19356.

[114] V. Lulevich, T. Zink, H.-Y. Chen, F.-T. Liu, G.-Y. Liu, Cell mechanics using atomic force microscopy-based single-cell compression, Langmuir 2006, 22(19), 8151-8155.

[115] D. Kirmizis, S. Logothetidis, Atomic force microscopy probing in the measurement of cell mechanics, Int. J. Nanomed. 2010, 5(137), 137-145.

[116] J. L. Hutter, J. Bechhoefer, Calibration of atomic-force microscope tips, Rev. Sci. Instrum. 1993, 64(7), 1868-1873. 
[117] R. Proksch, T. E. Schäffer, J. P. Cleveland, R. C. Callahan, M. B. Viani, Finite optical spot size and position corrections in thermal spring constant calibration, Nanotechnology 2004, 15(9), 1344-1350.

[118] H. J. Butt, M. Jaschke, Calculation of thermal noise in atomic force microscopy, Nanotechnology 1995, 6(1), 1-7.

[119] J. W. Kuhlmann, I. P. Mey, C. Steinem, Modulating the lateral tension of solvent-free pore-spanning membranes, Langmuir 2014, 30(27), 8186-8192.

[120] D. Norouzi, M. M. Müller, M. Deserno, How to determine local elastic properties of lipid bilayer membranes from atomic-force-microscope measurements: A theoretical analysis, Phys. Rev. E 2006, 74(6), 061914.

[121] P. Hansma, B. Drake, O. Marti, S. Gould, C. Prater, The scanning ionconductance microscope, Science 1989, 243(4891), 641-643.

[122] C.-C. Chen, Y. Zhou, L. A. Baker, Scanning ion conductance microscopy, Annu. Rev. Anal. Chem. 2012, 5(1), 207-228.

[123] B. Bhushan, H. Fuchs, Applied scanning probe methods II: Scanning probe microscopy techniques, Springer Berlin Heidelberg New York, 2006, 91-117.

[124] J. I. Goldstein, H. Yakowitz, Practical scanning electron microscopy: Electron and ion microprobe analysis, Plenum Press, New York, 1979, 21-29.

[125] T. E. Everhart, R. Thornley, Wide-band detector for micro-microampere low-energy electron currents, J. Sci. Instrum. 1960, 37(7), 246.

[126] N. Rodriguez, F. Pincet, S. Cribier, Giant vesicles formed by gentle hydration and electroformation: A comparison by fluorescence microscopy, Colloid. Surface. B. 2005, 42(2), 125-130.

[127] N. F. Morales-Penningston, J. Wu, E. R. Farkas, S. L. Goh, T. M. Konyakhina, J. Y. Zheng, W. W. Webb, G. W. Feigenson, GUV preparation and imaging: Minimizing artifacts, BBA - Biomembranes 2010, 1798(7), 1324-1332.

[128] J. C. Stachowiak, C. C. Hayden, D. Y. Sasaki, Steric confinement of proteins on lipid membranes can drive curvature and tubulation, Proc. Natl. Acad. Sci. U.S.A. 2010, 107(17), 7781-7786.

[129] T. Kirchhausen, Bending membranes, Nat. Cell Biol. 2012, 14(9), 906-908. 
[130] I. Langmuir, The adsorption of gases on plane surfaces of glass, mica and platinum, J. Am. Chem. Soc. 1918, 40(9), 1361-1403.

[131] J. Feder, I. Giaever, Adsorption of ferritin, J. Colloid Interf. Sci. 1980, 78(1), 144-154.

[132] J. Hyman, H. Chen, P. P. Di Fiore, P. De Camilli, A. T. Brunger, Epsin 1 undergoes nucleocytosolic shuttling and its Eps15 interactor NH2-terminal homology (ENTH) domain, structurally similar to Armadillo and HEAT repeats, interacts with the transcription factor promyelocytic leukemia $\mathrm{Zn} 2+$ finger protein (PLZF), J. Cell Biol. 2000, 149(3), 537-546.

[133] H. Neubacher, I. Mey, C. Carnarius, T. D. Lazzara, C. Steinem, Permeabilization assay for antimicrobial peptides based on pore-spanning lipid membranes on nanoporous alumina, Langmuir 2014, 30(16), 4767-4774.

[134] L. L. G. Schwenen, R. Hubrich, D. Milovanovic, B. Geil, J. Yang, A. Kros, R. Jahn, C. Steinem, Resolving single membrane fusion events on planar pore-spanning membranes, Sci. Rep. 2015, 5(12006), 1-15.

[135] T. Kaufeld, C. Steinem, C. F. Schmidt, Microporous device for local electric recordings on model lipid bilayers, J. Phys. D. Appl. Phys. 2015, 48(2), 025401 .

[136] A. Janshoff, C. Steinem, Mechanics of lipid bilayers: What do we learn from pore-spanning membranes?, BBA - Mol. Cell Res. 2015, 1853(11), 2977-2983.

[137] T. Kishimoto, Y. Sun, C. Buser, J. Liu, A. Michelot, D. G. Drubin, Determinants of endocytic membrane geometry, stability, and scission, Proc. Natl. Acad. Sci. U.S.A. 2011, 108(44), E979-E988.

[138] S. Steltenkamp, M. M. Müller, M. Deserno, C. Hennesthal, C. Steinem, A. Janshoff, Mechanical properties of pore-spanning lipid bilayers probed by atomic force microscopy, Biophys. J. 2006, 91(1), 217-226.

[139] M. Kocun, T. D. Lazzara, C. Steinem, A. Janshoff, Preparation of solventfree, pore-spanning lipid bilayers: Modeling the low tension of plasma membranes, Langmuir 2011, 27(12), 7672-7680. 
[140] C. H. Huang, P. Y. Hsiao, F. G. Tseng, S. K. Fan, C. C. Fu, R. L. Pan, Porespanning lipid membrane under indentation by a probe tip: A molecular dynamics simulation study, Langmuir 2011, 27(19), 11930-11942.

[141] I. Mey, M. Stephan, E. K. Schmitt, M. M. Müller, M. B. Amar, C. Steinem, A. Janshoff, Local membrane mechanics of pore-spanning bilayers, J. Am. Chem. Soc. 2009, 131(20), 7031-7039.

[142] T. Engel, The interaction of molecular and atomic oxygen with $\operatorname{Si}(100)$ and Si(111), Surf. Sci. Rep. 1993, 18(4), 93-144.

[143] J. Rheinlaender, T. E. Schäffer, Image formation, resolution, and height measurement in scanning ion conductance microscopy, J. Appl. Phys. 2009, 105(9), 094905.

[144] V. A. Lubarda, Mechanics of a liquid drop deposited on a solid substrate, Soft Matter 2012, 8(40), 10288-10297.

[145] S. S. Holkar, S. C. Kamerkar, T. J. Pucadyil, Spatial control of epsin-induced clathrin assembly by membrane curvature, J. Biol. Chem. 2015, 290(14), 14267-14276.

[146] A. A. Spector, M. A. Yorek, Membrane lipid composition and cellular function, J. Lipid Res. 1985, 26(9), 1015-1035.

[147] D.-H. Kweon, Y.-K. Shin, J. Y. Shin, J.-H. Lee, J.-B. Lee, J.-H. Seo, Y. S. Kim, Membrane topology of helix 0 of the epsin N-terminal homology domain, Mol. Cells 2006, 21(3), 428-435.

[148] C. L. Lai, C. C. Jao, E. Lyman, J. L. Gallop, B. J. Peter, H. T. McMahon, R. Langen, G. A. Voth, Membrane binding and self-association of the epsin N-terminal homology domain, J. Mol. Biol. 2012, 423(5), 800-817.

[149] T. Baumgart, B. R. Capraro, C. Zhu, S. L. Das, Thermodynamics and mechanics of membrane curvature generation and sensing by proteins and lipids, Annu. Rev. Phys. Chem. 2011, 62, 483-506.

[150] M. Gleisner, I. Mey, M. Barbot, C. Dreker, M. Meinecke, C. Steinem, Driving a planar model system into the 3rd dimension: Generation and control of curved pore-spanning membrane arrays, Soft Matter 2014, 10(33), 62286236. 
[151] B. Bailey, D. L. Farkas, D. L. Taylor, F. Lanni, Enhancement of axial resolution in fluorescence microscopy by standing-wave excitation, Nature 1993, 366(6450), 44-48.

[152] P. E. Hanninen, S. W. Hell, J. Salo, E. Soini, C. Cremer, Two-photon excitation 4Pi confocal microscope: Enhanced axial resolution microscope for biological research, Appl. Phys. Lett. 1995, 66(13), 1698-1700.

[153] R. Dimova, S. Aranda, N. Bezlyepkina, V. Nikolov, K. A. Riske, R. Lipowsky, A practical guide to giant vesicles. Probing the membrane nanoregime via optical microscopy, J. Phys. Condens. matter 2006, 18(28), S1151-S1176.

[154] O. Wesołowska, K. Michalak, J. Maniewska, A. B. Hendrich, Giant unilamellar vesicles - a perfect tool to visualize phase separation and lipid rafts in model systems, Acta Biochim. Pol. 2009, 56(1/2009), 33-39.

[155] H. D. Ou-Yang, M.-T. Wei, Complex fluids: Probing mechanical properties of biological systems with optical tweezers, Annu. Rev. Phys. Chem. 2010, 61, 421-40.

[156] J. B. Manneville, P. Bassereau, S. Ramaswamy, J. Prost, Active membrane fluctuations studied by micropipet aspiration, Phys. Rev. E 2001, 64, 021908.

[157] S. Dieluweit, A. Csiszár, W. Rubner, J. Fleischhauer, S. Houben, R. Merkel, Mechanical properties of bare and protein-coated giant unilamellar phospholipid vesicles. A comparative study of micropipet aspiration and atomic force microscopy, Langmuir 2010, 26(13), 11041-11049.

[158] E. Sackmann, R. F. Bruinsma, Cell adhesion as wetting transition?, ChemPhysChem. 2002, 3(3), 262-269.

[159] A. Ramachandran, T. H. Anderson, L. G. Leal, J. N. Israelachvili, Adhesive interactions between vesicles in the strong adhesion limit, Langmuir 2011, $27(1), 59-73$.

[160] F. Brochard, P. De Gennes, P. Pfeuty, Surface tension and deformations of membrane structures: Relation to two-dimensional phase transitions, J. Phys. - Paris 1976, 37(10), 1099-1104.

[161] W. Helfrich, R. M. Servuss, Undulations, steric interaction and cohesion of fluid membranes, II Nuovo Cim. D 1984, 3(1), 137-151. 
[162] S. T. Milner, S. A. Safran, Dynamical fluctuations of droplet microemulsions and vesicles, Phys. Rev. A 1987, 36(9), 4371-4379.

[163] D. Marsh, Renormalization of the tension and area expansion modulus in fluid membranes, Biophys. J. 1997, 73(2), 865-869.

[164] E. Evans, W. Rawicz, Entropy-driven tension and bending elasticity in condensed-fluid membranes, Phys. Rev. Lett. 1990, 64(17), 2094-2097.

[165] D. W. Deamer, J. Bramhall, Permeability of lipid bilayers to water and ionic solutes, Chem. Phys. Lipids 1986, 40(2-4), 167-188.

[166] A.-L. Bernard, M.-A. Guedeau-Boudeville, L. Jullien, J. M. Di Meglio, Strong adhesion of giant vesicles on surfaces: Dynamics and permeability, Langmuir 2000, 16(17), 6809-6820.

[167] J. O. Rädler, T. J. Feder, H. H. Strey, E. Sackmann, Fluctuation analysis of tension-controlled undulation forces between giant vesicles and solid substrates, Phys. Rev. E 1995, 51(5), 4526-4536.

[168] W. Rawicz, B. Smith, T. McIntosh, S. Simon, E. Evans, Elasticity, strength, and water permeability of bilayers that contain raft microdomain-forming lipids, Biophys. J. 2008, 94(12), 4725-4736.

[169] W. Rawicz, K. Olbrich, T. McIntosh, D. Needham, E. Evans, Effect of chain length and unsaturation on elasticity of lipid bilayers, Biophys. J. 2000, 79(1), 328-339.

[170] J. Rädler, E. Sackmann, Imaging optical thicknesses and separation distances of phospholipid vesicles at solid surfaces, J. Phys. II 1993, 3(5), 727-748.

[171] O. Shindell, N. Mica, M. Ritzer, V. Gordon, Specific adhesion of membranes simultaneously supports dual heterogeneities in lipids and proteins, Phys. Chem. Chem. Phys. 2015, 17(24), 15598-15607.

[172] S. F. Fenz, K. Sengupta, Giant vesicles as cell models, Integr. Biol. 2012, 4(9), 982-995.

[173] A. Albersdörfer, T. Feder, E. Sackmann, Adhesion-induced domain formation by interplay of long-range repulsion and short-range attraction force: A model membrane study, Biophys. J. 1997, 73(1), 245-257. 
[174] E. Evans, Entropy-driven tension in vesicle membranes and unbinding of adherent vesicles, Langmuir 1991, 7(9), 1900-1908.

[175] T. Gruhn, T. Franke, R. Dimova, R. Lipowsky, Novel method for measuring the adhesion energy of vesicles, Langmuir 2007, 23(10), 5423-5429.

[176] Y.-h. Wang, A. Collins, L. Guo, K. B. Smith-Dupont, F. Gai, T. Svitkina, P. A. Janmey, Divalent cation-induced cluster formation by polyphosphoinosited in model membranes, J. Am. Chem. Soc. 2012, 134(7), 3387-3395.

[177] L. A. Flanagan, C. C. Cunningham, J. Chen, G. D. Prestwich, K. S. Kosik, P. A. Janmey, The structure of divalent cation-induced aggregates of PIP2 and their alteration by gelsolin and tau, Biophys. J. 1997, 73(3), 1440-1447.

[178] W. G. Ellenbroek, Y.-H. Wang, D. A. Christian, D. E. Discher, P. A. Janmey, A. J. Liu, Divalent cation-dependent formation of electrostatic PIP2 clusters in lipid monolayers, Biophys. J. 2011, 101(9), 2178-2184.

[179] Z. T. Graber, A. Gericke, E. E. Kooijman, Phosphatidylinositol-4,5bisphosphate ionization in the presence of cholesterol, calcium or magnesium ions, Chem. Phys. Lipids 2014, 182, 62-72.

[180] M. Frick, K. Schmidt, B. J. Nichols, Modulation of lateral diffusion in the plasma membrane by protein density, Curr. Biol. 2007, 17(5), 462-467.

[181] V. C. Coffman, J.-Q. Wu, Counting protein molecules using quantitative fluorescence microscopy, Trends Biochem. Sci. 2012, 37(11), 499-506.

[182] Y. Sugiyama, I. Kawabata, K. Sobue, S. Okabe, Determination of absolute protein numbers in single synapses by a GFP-based calibration technique, Nat. Methods 2005, 2(9), 677-684.

[183] W. J. Galush, J. A. Nye, J. T. Groves, Quantitative fluorescence microscopy using supported lipid bilayer standards, Biophys. J. 2008, 95(5), 2512-2519.

[184] N. Kučerka, J. F. Nagle, J. N. Sachs, S. E. Feller, J. Pencer, A. Jackson, J. Katsaras, Lipid bilayer structure determined by the simultaneous analysis of neutron and X-ray scattering data, Biophys. J. 2008, 95(5), 2356-2367.

[185] Y. F. Dufrêne, T. Boland, J. W. Schneider, W. R. Barger, G. U. Lee, Characterization of the physical properties of model biomembranes at the nanometer scale with the atomic force microscope, Faraday Discuss. 1998, 111, 79-94. 
[186] G. Drin, B. Antonny, Amphipathic helices and membrane curvature, FEBS Lett. 2010, 584(9), 1840-1847.

[187] N. Kučerka, S. Tristram-Nagle, J. F. Nagle, Structure of fully hydrated fluid phase lipid bilayers with monounsaturated chains, J. Membrane Biol. 2006, 208(3), 193-202.

[188] D. H. Boal, Mechanics of the cell, 2nd edition, Cambridge University Press, Cambridge, 2012, 155.

[189] H. Bouvrais, P. Méléard, T. Pott, K. J. Jensen, J. Brask, J. H. Ipsen, Softening of POPC membranes by magainin, Biophys. Chem. 2008, 137(1), 7-12.

[190] S. Tristram-Nagle, J. F. Nagle, HIV-1 fusion peptide decreases bending energy and promotes curved fusion intermediates, Biophys. J. 2007, 93(6), 20482055.

[191] E. I. Settles, A. F. Loftus, A. N. McKeown, R. Parthasarathy, The vesicle trafficking protein Sar1 lowers lipid membrane rigidity, Biophys. J. 2010, 99(5), 1539-1545.

[192] S. Rozovsky, M. B. Forstner, H. Sondermann, J. T. Groves, Single molecule kinetics of ENTH binding to lipid membranes, J. Phys. Chem. B 2012, 116(17), 5122-5131.

[193] V. K. Bhatia, N. S. Hatzakis, D. Stamou, A unifying mechanism accounts for sensing of membrane curvature by BAR domains, amphipathic helices and membrane-anchored proteins, Semin. Cell Dev. Biol. 2010, 21(4), 381-390.

[194] W. T. Hsieh, C. J. Hsu, B. R. Capraro, T. Wu, C. M. Chen, S. Yang, T. Baumgart, Curvature sorting of peripheral proteins on solid-supported wavy membranes, Langmuir 2012, 28(35), 12838-12843.

[195] T. R. Graham, M. M. Kozlov, Interplay of proteins and lipids in generating membrane curvature, Curr. Opin. Cell Biol. 2010, 22(4), 430-436.

[196] G. Drin, J.-F. Casella, R. Gautier, T. Boehmer, T. U. Schwartz, B. Antonny, A general amphipathic alpha-helical motif for sensing membrane curvature, Nat. Struct. Mol. Biol. 2007, 14(2), 138-146. 
[197] R. B. Cornell, S. G. Taneva, Amphipathic helices as mediators of the membrane interaction of amphitropic proteins, and as modulators of bilayer physical properties, Curr. Protein Pept. Sc. 2006, 7(6), 539-552.

[198] N. S. Hatzakis, V. K. Bhatia, J. Larsen, K. L. Madsen, P.-Y. Bolinger, A. H. Kunding, J. Castillo, U. Gether, P. Hedegård, D. Stamou, How curved membranes recruit amphipathic helices and protein anchoring motifs, Nat. Chem. Biol. 2009, 5(11), 835-841.

[199] H. Cui, E. Lyman, G. A. Voth, Mechanism of membrane curvature sensing by amphipathic helix containing proteins, Biophys. J. 2011, 100(5), 1271-1279.

[200] B. Nuscher, F. Kamp, T. Mehnert, S. Odoy, C. Haass, P. J. Kahle, K. Beyer, $\alpha$-synuclein has a high affinity for packing defects in a bilayer membrane: A thermodynamics study, J. Biol. Chem. 2004, 279(21), 21966-21975.

[201] J. A. Gazzara, M. C. Phillips, S. Lund-Katz, M. N. Palgunachari, J. P. Segrest, G. M. Anantharamaiah, W. V. Rodrigueza, J. W. Snow, Effect of vesicle size on their interaction with class A amphipathic helical peptides, J. Lipid Res. 1997, 38(10), 2147-2154.

[202] C. Huang, J. T. Mason, Geometric packing constraints in egg phosphatidylcholine vesicles, Proc. Natl. Acad. Sci. U.S.A. 1978, 75(1), 308-310.

[203] S. M. Christensen, D. G. Stamou, Sensing-applications of surface-based single vesicle arrays, Sensor Rev. 2010, 10(12), 11352-11368.

[204] F. Campelo, H. T. McMahon, M. M. Kozlov, The hydrophobic insertion mechanism of membrane curvature generation by proteins, Biophys. J. 2008, 95(5), 2325-2339.

[205] R. V. Stan, Structure of caveolae, BBA - Mol. Cell Res. 2005, 1746(3), 334-348.

[206] M. Fernández-Suárez, A. Y. Ting, Fluorescent probes for super-resolution imaging in living cells, Nat. Rev. Mol. Cell Biol. 2008, 9(12), 929-943.

[207] B. O. Leung, K. C. Chou, Review of super - resolution fluorescence microscopy for biology, Appl. Spectrosc. 2011, 65(9), 967-980.

[208] N. C. Gauthier, T. A. Masters, M. P. Sheetz, Mechanical feedback between membrane tension and dynamics, Trends Cell Biol. 2012, 22(10), 527-535. 
[209] E. Schäfer, T.-T. Kliesch, A. Janshoff, Mechanical properties of giant liposomes compressed between two parallel plates: Impact of artificial actin shells, Langmuir 2014, 29(33), 10463-10474.

[210] R. P. Rand, A. C. Burton, Mechanical properties of the red cell membrane. I. Membrane stiffness and intracellular pressure, Biophys. J. 1964, 4(2), 115135.

[211] J. Dai, M. P. Sheetz, X. Wan, C. E. Morris, Membrane tension in swelling and shrinking molluscan neurons, J. Neurosci. 1998, 18(17), 6681-6692.

[212] D. Frese, S. Steltenkamp, S. Schmitz, C. Steinem, In situ generation of electrochemical gradients across pore-spanning membranes, RSC Adv. 2013, 3(36), 15752-15761.

[213] M. Kocun, A. Janshoff, Pulling tethers from pore-spanning bilayers: Towards simultaneous determination of local bending modulus and lateral tension of membranes, Small 2012, 8(6), 847-851.

[214] T. Ando, T. Uchihashi, N. Kodera, D. Yamamoto, A. Miyagi, M. Taniguchi, H. Yamashita, High-speed AFM and nano-visualization of biomolecular processes, Pflueg. Arch. J. Phy. 2008, 456(1), 211-225.

[215] T. Ando, T. Uchihashi, N. Kodera, High-speed AFM and applications to biomolecular systems, Annu. Rev. Biophys. 2013, 42, 393-414.

[216] H.-G. Döbereiner, G. Gompper, C. K. Haluska, D. M. Kroll, P. G. Petrov, K. A. Riske, Advanced flicker spectroscopy of fluid membranes, Phys. Rev. Lett. 2003, 91(4), 048301.

[217] C. Esposito, A. Tian, S. Melamed, C. Johnson, S.-Y. Tee, T. Baumgart, Flicker spectroscopy of thermal lipid bilayer domain boundary fluctuations, Biophys. J. 2007, 93(9), 3169-3181.

[218] G. Popescu, T. Ikeda, K. Goda, C. A. Best-Popescu, M. Laposata, S. Manley, R. R. Dasari, K. Badizadegan, M. S. Feld, Optical measurement of cell membrane tension, Phys. Rev. Lett. 2006, 97(21), 1-4.

[219] D. R. Stabley, C. Jurchenko, S. S. Marshall, K. S. Salaita, Visualizing mechanical tension across membrane receptors with a fluorescent sensor, Nat. Methods 2011, 9(1), 64-67. 
[220] M. E. Solmaz, S. Sankhagowit, R. Biswas, C. A. Mejia, M. L. Povinelli, N. Malmstadt, Optical stretching as a tool to investigate the mechanical properties of lipid bilayers, RSC Adv. 2013, 3(37), 16632-16638.

[221] E. K. Hoffmann, I. H. Lambert, S. F. Pedersen, Physiology of cell volume regulation in vertebrates, Physiol. Rev. 2009, 89(1), 193-277.

[222] Y. Tao, M. Esashi, Local formation of macroporous silicon through a mask, J. Micromech. Microeng. 2004, 14(10), 1411-1415.

[223] V. Lehmann, U. Grüning, The limits of macropore array fabrication, Thin Solid Films 1997, 297(1), 13-17.

[224] S. Unnikrishnan, H. Jansen, E. Berenschot, M. Elwenspoek, Wafer scale nano-membranes supported on a silicon microsieve using thin-film transfer technology, J. Micromech. Microeng. 2008, 18(6), 064005.

[225] G. Vasquez, E. Alvarez, J. M. Navaza, Tension of alcohol + Water from 20 to $50{ }^{\circ} \mathrm{C}, \mathrm{J}$. Chem. Eng. Data 1995, 40(3), 611-614.

[226] L. I. Rolo, A. I. Caço, A. J. Queimada, I. M. Marrucho, J. A. P. Coutinho, Surface tension of heptane, decane, hexadecane, eicosane, and some of their binary mixtures, J. Chem. Eng. Data 2002, 47(6), 1442-1445.

[227] D. R. Slochower, P. J. Huwe, R. Radhakrishnan, P. A. Janmey, Quantum and all-atom molecular dynamics simulations of protonation and divalent ion binding to phosphatidylinositol 4,5-bisphosphate (PIP2), J. Phys. Chem. B 2013, 117(28), 8322-8329.

[228] N. Kučerka, M.-P. Nieh, J. Katsaras, Fluid phase lipid areas and bilayer thicknesses of commonly used phosphatidylcholines as a function of temperature, BBA - Biomembranes 2011, 1808(11), 2761-2771.

[229] E. A. Evans, V. A. Parsegian, Energetics of membrane deformation and adhesion in cell and vesicle aggregation, Ann. N. Y. Acad. Sci. 1983, 416(1), 13-33.

[230] R. Servuss, W. Helfrich, Mutual adhesion of lecithin membranes at ultralow tensions, J. Phys. - Paris 1989, 50(7), 809-827.

[231] R. Lipowsky, U. Seifert, Adhesion of vesicles and membranes, Mol. Cryst. Liq. Cryst. 1991, 202(1), 17-25. 
[232] J. Ekeroth, P. Konradsson, F. Höök, Bivalent-ion-mediated vesicle adsorption and controlled supported phospholipid bilayer formation on molecular phosphate and sulfate layers on gold, Langmuir 2002, 18(21), 7923-7929.

[233] B. Seantier, B. Kasemo, Influence of mono- and divalent ions on the formation of supported phospholipid bilayers via vesicle adsorption, Langmuir 2009, 25(10), 5767-5772.

[234] D. A. Noppl-Simson, D. Needham, Avidin-biotin interactions at vesicle surfaces: Adsorption and binding, cross-bridge formation, and lateral interactions, Biophys. J. 1996, 70(3), 1391-1401.

[235] A. Berthaud, F. Quemeneur, M. Deforet, P. Bassereau, F. Brochard-Wyart, S. Mangenot, Spreading of porous vesicles subjected to osmotic shocks: The role of aquaporins, Soft Matter 2016.

[236] A. Zocchi, A. M. Jobé, J. M. Neuhaus, T. R. Ward, Expression and purification of a recombinant avidin with a lowered isoelectric point in Pichia pastoris, Protein Expres. Purif. 2003, 32(2), 167-174.

[237] D. Raucher, T. Stauffer, W. Chen, K. Shen, S. Guo, J. D. York, M. P. Sheetz, T. Meyer, Phosphatidylinositol 4,5-bisphosphate functions as a second messenger that regulates cytoskeleton-plasma membrane adhesion, Cell 2000, 100(2), 221-228.

[238] R. Simson, E. Wallraff, J. Faix, J. Niewöhner, G. Gerisch, E. Sackmann, Membrane bending modulus and adhesion energy of wild-type and mutant cells of Dictyostelium lacking talin or cortexillins, Biophys. J. 1998, 74(1), 514-522.

[239] R. M. Hochmuth, W. D. Marcus, Membrane tethers formed from blood cells with available area and determination of their adhesion energy, Biophys. J. 2002, 82(6), 2964-2969.

[240] S. Pierrat, F. Brochard-Wyart, P. Nassoy, Enforced detachment of red blood cells adhering to surfaces: Statics and dynamics, Biophys. J. 2004, 87(4), 2855-2869.

[241] V. T. Moy, Y. Jiao, T. Hillmann, H. Lehmann, T. Sano, Adhesion energy of receptor-mediated interaction measured by elastic deformation, Biophys. J. 1999, 76(3), 1632-1638. 
[242] N. M. Green, Avidin and streptavidin, Method. Enzymol. 1990, 184, 51-67.

[243] P. Shchelokovskyy, S. Tristram-Nagle, R. Dimova, Effect of the HIV-1 fusion peptide on the mechanical properties and leaflet coupling of lipid bilayers, New J. Phys. 2011, 13(2), 025004.

[244] H. Bouvrais, L. Duelund, J. H. Ipsen, Buffers affect the bending rigidity of model lipid membranes, Langmuir 2014, 30(1), 13-16.

[245] B. R. Capraro, Y. Yoon, W. Cho, T. Baumgart, Curvature sensing by the epsin N-terminal homology domain measured on cylindrical lipid membrane tethers, J. Am. Chem. Soc. 2010, 132(4), 1200-1201.

[246] S. Leibler, Curvature instability in membranes, J. Phys. - Paris 1986, 47(3), 507-516.

[247] J. F. Nagle, S. Tristram-Nagle, Structure of lipid bilayers, Biochim. Biophys. Acta 2000, 1469(3), 159-195.

[248] R. W. Tourdot, N. Ramakrishnan, R. Radhakrishnan, Defining the free-energy landscape of curvature-inducing proteins on membrane bilayers, Phys. Rev. E 2014, 90(2), 022717.

[249] R. W. Tourdot, N. Ramakrishnan, T. Baumgart, R. Radhakrishnan, Application of a free energy landscape approach to study tension dependent bilayer tubulation mediated by curvature, Phys. Rev. E 2015, 92(4), 042715.

[250] J. W. Donovan, K. D. Ross, Increase in the stability of avidin produced by binding of biotin. A differential scanning calorimetric study of denaturation by heat, Biochemistry 1973, 12(3), 512-517.

[251] U. Piran, W. J. Riordan, Dissociation rate constant of the biotin-streptavidin complex, J. Immunol. Methods 1990, 133(1), 141-143.

[252] W. Helfrich, Elastic properties of lipid bilayers - theory and possible experiments, Z. Naturforsch. C 1973, 28(11-12), 693-703.

[253] P. Sens, M. S. Turner, Theoretical model for the formation of caveolae and similar membrane invaginations, Biophys. J. 2004, 86(4), 2049-2057. 
[254] R. Lipowsky, Spontaneous tubulation of membranes and vesicles reveals membrane tension generated by spontaneous curvature, Faraday Discuss. 2013, 161, 305-331.

[255] M. J. Taylor, D. Perrais, C. J. Merrifield, A high precision survey of the molecular dynamics of mammalian clathrin-mediated endocytosis, PLoS Biol. 2011, 9(3), e1000604.

[256] S. F. Fenz, A. S. Smith, R. Merkel, K. Sengupta, Inter-membrane adhesion mediated by mobile linkers: Effect of receptor shortage, Soft Matter 2011, $7(3), 952-962$.

[257] M. Simunovic, G. A. Voth, Membrane tension controls the assembly of curvature-generating proteins, Nat. Commun. 2015, 6(7219), 1-8.

[258] S. Aghamohammadzadeh, K. R. Ayscough, Differential requirements for actin during yeast and mammalian endocytosis, Nat. Cell Biol. 2009, 11(8), 1039-1042. 


\section{A Appendix}

\section{A.1 List of Figures}

1.1 Schematic illustration of the different types of curvature of a membrane bud and a vesicle. . . . . . . . . . . . . . 2

1.3 Schematic illustration of the formation and fission of an endocytotic vesicle. ........................ 6

1.4 Schematic illustration of processes regulating membrane tension. . . 8

1.5 Crystal structure of ENTH and the ENTH-I $(1,4,5) \mathrm{P}_{3}$ complex. . . . . 10

3.1 Plot of measured osmolality vs. theoretical osmolality for $\mathrm{NaCl}$ and

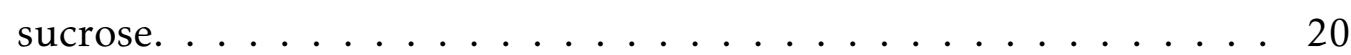

3.2 Point spread function. . . . . . . . . . . . . . . . . . . 29

3.3 Digitalization of an analog signal. . . . . . . . . . . . . 30

3.4 Schematic illustration of the operation principle of confocal laser scanning microscopy. . . . . . . . . . . . . . . 32

3.5 Schematic illustration of the operation principle of spinning disc microscopy. . . . . . . . . . . . . . . 33

3.6 Schematic illustration of out-of-focus light resulting in pinhole

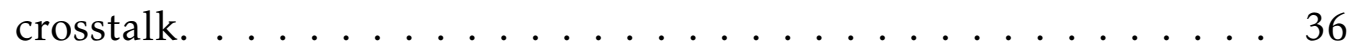

3.7 Transmission spectra of selected emission filters equipped to the spinning disc microscope. . . . . . . . . . . . . . . . . 37

3.8 Signal-to-noise ratio as a function of the number of photons per pixel. 39

3.9 Dynamic range as a function of the applied EM gain for different readout speeds. . . . . . . . . . . . . . . . 41

3.10 Schematic illustration of a prism attached to a gold layer and the excitation of surface plasmons. . . . . . . . . . . . . 45 
3.11 Schematic illustration of the correlation of surface plasmon excitation and the measured reflectivity as a function of time. . . . . . . . 46

3.12 Schematic illustration of the light pathway in reflectometric interference spectroscopy. . . . . . . . . . . . . . . . . . 48 48

3.13 Schematic illustration of the interaction potential between cantilever and sample. . . . . . . . . . . . . . . . 52

3.14 Schematic illustration of the measurement of a force-distance curve. 53

3.15 Current as a function of the tip-sample distance for a typical pipette

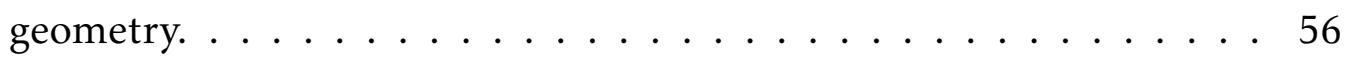

3.16 Schematic illustration of the operation principle of SICM. . . . . . . 57

3.17 Schematic illustration of the operation principle and types of signals produced in scanning electron microscopy. . . . . . . . . . . . . . 59

3.18 Schematic illustration of the script used for data evaluation to determine the GUV geometry. . . . . . . . . . . . . . 61

3.19 Humidity chamber and flattening of GUVs as a function of time for non-isoosmolaric conditions. . . . . . . . . . . . 63

3.20 Contact area of adhered GUVs increases as a function of the $\mathrm{NaCl}$ concentration. . . . . . . . . . . . . . . 64

$4.1 \quad$ Representative time resolved change in optical thickness of a typical experiment observed by means of RIfS. . . . . . . . . . . . . 66

4.2 Isotherm of ENTH binding to a planar solid-supported lipid bilayer measured by means of RIfS. . . . . . . . . . . . . . . . . 67

4.3 Representative time resolved reflectivity change of a typical experiment obtained by means of SPR spectroscopy. . . . . . . . . . . . . 69

4.4 Binding isotherms for ENTH binding to LUVs measured by SPR spectroscopy. . . . . . . . . . . . . . . 70

4.5 Scheme and corresponding confocal fluorescence microscopy images of pore-spanning membranes on a porous silicon substrate. . . . . . 72

4.6 Radii of pore-spanning membranes measured for different osmolarity gradients from fluorescence microscopy images. . . . . . 73

4.7 Confocal fluorescence microscopy images of a membrane patch before and after application of an osmolarity gradient. . . . . . . . 74

4.8 Scanning ion conductance microscopy image of protruded porespanning DPhPC membranes after application of an osmolarity

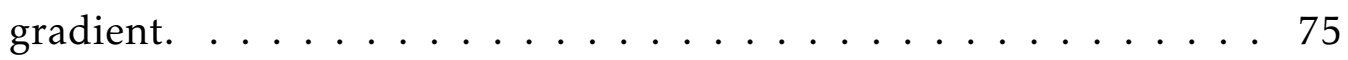


4.9 Evaluation procedure for the determination the height of protruded pore-spanning membranes. . . . . . . . . . . . . 75

4.10 Heights of protruded pore-spanning POPC and DPhPC membranes measured for different applied osmolarity gradients and calculated osmotic pressure corresponding to the measured membrane heights. 78

4.11 Time series of confocal fluorescence microscopy images of protruded pore-spanning membranes after addition of ENTH. . . . . . . . . . 79

4.12 Normalized mean radius and number of pore-spanning membranes with and without $\mathrm{PIP}_{2}$ as a function of time. . . . . . . . . 81

4.13 Surface rendering of SDCLM images of growing and shrinking protruded PSMs after application of an osmolarity gradient of $19 \mathrm{mOsmol} / \mathrm{L}$ and subsequent addition of ENTH as a function of time. 82

4.14 Calculated heights of pore-spanning membranes on pores with a depth of $8 \mu \mathrm{m}$ as a function of the applied osmolarity gradient, simulated for three different membrane tensions and osmolarities used for PSM formation. . . . . . . . . . . . . . . . . . . . 85

4.15 SDCLM images of micrometer-sized pore-spanning membranes after application of different osmolarity gradients. . . . . . . . . . 87

4.16 Evaluation procedure and heights of micrometer-sized porespanning membranes as a function of the applied osmolarity gradient. 89

4.17 Histogram of lateral membrane tensions of protruded pore-spanning membranes obtained by means of fluorescence microscopy. . . . . . 90

4.18 Scanning electron microscopy image of a cross section of a porous

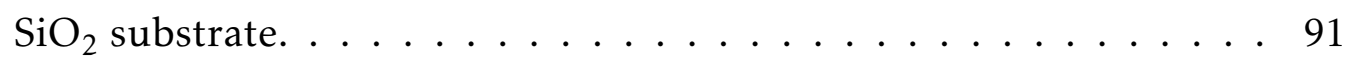

4.19 Force volume image of pore-spanning membranes after application of an osmolarity gradient and corresponding representative forcedistance curves. . . . . . . . . . . . . . . . . . 92

4.20 Scheme of a GUV on a surface before and after adhesion. . . . . . . 96

4.21 Fractional area and radius of an adhered vesicle as a function of the contact radius. . . . . . . . . . . . . . . . . . . . . . . 98

4.22 Resulting membrane tension of GUVs adhering to a substrate as a function of $r_{\mathrm{i}} / r_{\mathrm{ad}}$, calculated for different pre-tensions. . . . . . . . . 99

4.23 Representative 3D reconstructions of GUVs adhering to an avidincoated glass surface at different $\mathrm{Mg}^{2+}$ concentrations. . . . . . . . . . 101 
4.24 Ratio of contact area radius to radius of the adhered vesicle at different $\mathrm{Mg}^{2+}$ concentrations. . . . . . . . . . . . . . . 102

4.25 Lateral membrane tension of GUVs adhering to an avidin-coated glass surface at different $\mathrm{Mg}^{2+}$ concentrations. . . . . . . . . . 103

4.26 Adhesion energies of GUVs adhering to an avidin-coated glass surface at different $\mathrm{Mg}^{2+}$ concentrations. . . . . . . . . . . . . 104

4.27 Overlay of SDCLM $z$-profiles of GUVs labeled with Atto488-DPPE and Bodipy-TMR-PIP ${ }_{2}$ on an avidin-coated glass surface imaged for different buffers and $\mathrm{Mg}^{2+}$ concentrations. . . . . . . . . . . 107

4.28 Representative illustration of the evaluation procedure for quantification of the fluorescence intensity of GUV membranes. . . . . . . 109

4.29 Plot of absolute fluorescence intensity of GUVs as a function of the

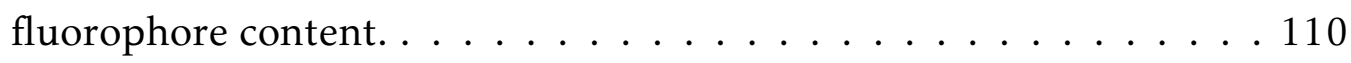

4.30 Quantification of the intensity of ENTH-Atto594 binding to GUVs. 111

4.31 Fluorescence microscopy image of specific and unspecific binding of ENTH-Atto594 and protein stability as a function of time. . . . . 112

4.32 Time series confocal fluorescence microscopy images of an adhered GUV in sucrose buffer containing $2 \mathrm{~mm} \mathrm{Mg}^{2+}$ flattening after ENTH

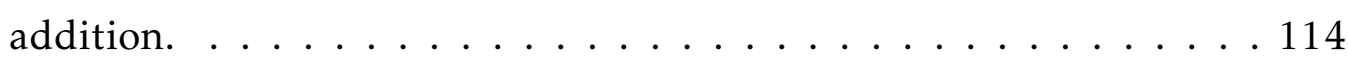

4.33 Time series confocal fluorescence microscopy images of an adhered GUV in sucrose buffer containing $0.5 \mathrm{mM} \mathrm{Mg}^{2+}$ forming membrane tubules after ENTH addition. . . . . . . . . . . . . . . 115

$4.34 r_{\mathrm{i}} / r_{\text {ad }}$ of GUVs adhering to an avidin-coated glass surface in sucrose buffer with different $\mathrm{Mg}^{2+}$ concentrations measured before and after ENTH addition. . . . . . . . . . . . . . . . . 116

5.1 Schematic illustration of the exposure of the hydrophobic bilayer core of a planar, curved and tensed membrane. . . . . . . . . . 125

5.2 Schematic illustration of the resulting membrane height of protruded pore-spanning membranes after application of an osmolarity gradient for different membrane tensions and pore geometries. . . . 132

5.3 Simulated heights of pore-spanning membranes as a function of the applied osmolarity gradient and simulated reduction in height of protruded pore-spanning membranes as a function of the lateral membrane tension. . . . . . . . . . . . . . . . . . . 134 
5.4 Schematic illustration of ENTH binding decreasing the lateral tension of protruded pore-spanning membranes resulting in growth of the protruded pore-spanning membranes. . . . . . . . . . 136

5.5 Different regimes of the adhesion process of a vesicle to a surface. . 139

5.6 Membrane thinning, enlarged spacing of the lipid monolayers and disordered lipid packing as a result of helix insertion. . . . . . . . . 147

5.7 Schematic illustration of ENTH binding to a lipid bilayer containing

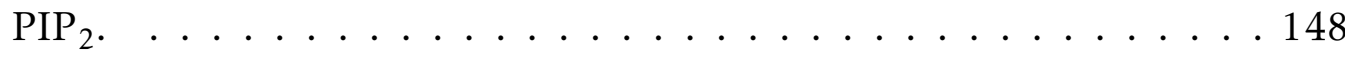

5.8 Schematic illustration of ENTH binding to adhered GUVs resulting in either formation of membrane tubes or flattening of the vesicle. . 148

\section{A.2 List of Schemes}

3.1 Structure of POPC. . . . . . . . . . . . . . . . . 21

3.2 Structure of POPE . . . . . . . . . . . . . . . 21

3.3 Structure of DOPC. . . . . . . . . . . . . . . 22

3.4 Structure of DOPE. . . . . . . . . . . . . . . 22

3.5 Structure of DPhPC. . . . . . . . . . . . . . . . . . 23

3.6 Structure of cap-biotin-DOPE. . . . . . . . . . . . . . 23

3.7 Structure of cholesterol. . . . . . . . . . . . . . . . 23

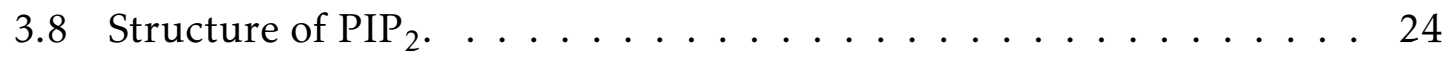

\section{A.3 List of Tables}

3.1 Properties of compounds used as markers for fluorescence microscopy. 25

3.2 Flow rates and durations of the different steps of a SPR spectroscopy

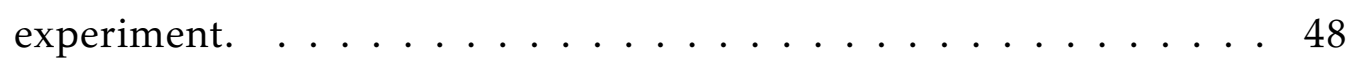

$3.3 \quad$ Experimental parameters used for the SICM experiments. . . . . . . 58

5.1 Dissociation constants determined by SPR spectroscopy, RIfS and SDCLM. . . . . . . . . . . . . . . . . 122 


\section{A.4 Coworkers}

Christian Fricke performed the reflectometric interference spectroscopy experiments for the determination of the dissociation constant of ENTH binding to planar solid supported membranes. Porous $\mathrm{SiO}_{2}$ substrates were imaged by Jeremias Sibold using scanning electron microscopy. In addition, he conducted the atomic force microscopy experiments to quantify the lateral membrane tension and height of protruded pore-spanning membranes as a function of the applied osmolarity gradient.

\section{A.5 Sequence of ENTH}

Properties of ENTH were calculated based on the sequence using the tool ProtParam. 48, 70]

\section{Sequence of ENTH}

1 HNYSEAEIKV REATSNDPWG PSSSLMSEIA DLTYNVVAFS

41 EIMSMIWKRL NDHGKNWRHV YKAMTLMEYL IKTGSERVSQ

81 QCKENMYAVQ TLKDFQYVDR DGKDQGVNVR EKAKQLVALL

121 RDEDRLREER AHALKTKEKL AQTA

\section{A.6 Abbreviations}

$\begin{array}{ll}\text { A } & \text { area } \\ \text { AC } & \text { alternating current } \\ \text { AFM } & \text { atomic force microscope } \\ \text { AOTF } & \text { acousto optic tunable filter } \\ \text { BSA } & \text { bovine serum albumin } \\ \text { Chol } & \text { cholesterol } \\ \text { CLSM } & \text { confocal laser scanning microscope } \\ d & \text { diameter, resolution } \\ \text { DHPE } & 1,2 \text {-dihexadecanoyl-sn-glycero-3-phosphoethanolamine } \\ \text { DMSO } & \text { dimethyl sulfoxide }\end{array}$




\begin{tabular}{|c|c|}
\hline DOL & degree of labeling \\
\hline DOPC & 1,2-dioleoyl-sn-glycero-3-phosphocholine \\
\hline DOPE & 1,2-dioleoyl-sn-glycero-3-phosphoethanolamine \\
\hline$\epsilon$ & dielectric constant \\
\hline ENTH & epsin N-terminal homology domain \\
\hline ENTH-Alexa 488 & epsin N-terminal homology domain labeled with \\
\hline ENTH-Atto594 & $\begin{array}{l}\text { Alexa } 488 \text { carboxylic acid TFP ester } \\
\text { epsin N-terminal homology domain labeled with } \\
\text { Atto594-maleimide }\end{array}$ \\
\hline EMCCD & electron multiplying charge coupled device \\
\hline$f$ & frequency \\
\hline$F$ & force, energy \\
\hline F-actin & filamentous actin \\
\hline FWHM & full width half maximum \\
\hline$\gamma \mathrm{ad}$ & adhesion energy per unit area \\
\hline GUV & giant unilamellar vesicle \\
\hline$h$ & height \\
\hline HEPES & 2-[4-(2-hydroxyethyl)piperazin-1-yl]ethanesulfonic acid \\
\hline$I$ & intensity \\
\hline ITO & indium tin oxide \\
\hline$\kappa$ & bending rigidity \\
\hline$k$ & spring constant, momentum \\
\hline$k_{\mathrm{B}}$ & Boltzmann constant \\
\hline$K_{A}$ & area compressibility modulus \\
\hline$K_{\mathrm{D}}$ & dissociation constant \\
\hline LUV & large unilamellar vesicle \\
\hline$\lambda$ & wave length \\
\hline$M$ & molar mass \\
\hline M & molar \\
\hline $\min$ & minute \\
\hline MOPS & 3-(N-morpholino)propanesulfonic acid \\
\hline$n$ & number, amount of substance \\
\hline
\end{tabular}




\begin{tabular}{|c|c|}
\hline$N$ & noise \\
\hline$N A$ & numerical aperture \\
\hline$O$ & osmolarity \\
\hline OT & optical thickness \\
\hline$\Pi$ & osmotic pressure \\
\hline $\mathrm{PIP}_{2}$ & phosphatidylinositol-4,5-bisphosphate \\
\hline POPC & 1-palmitoyl-2-oleoyl-sn-glycero-3-phosphocholine \\
\hline POPE & 1-palmitoyl-2-oleoyl-sn-glycero-3-phosphoethanolamine \\
\hline PSF & point spread function \\
\hline PSM & pore-spanning membrane \\
\hline RIfS & reflectometric interference spectroscopy \\
\hline rpm & rounds per minute \\
\hline$r$ & radius, Fresnel reflection coefficient \\
\hline$R$ & reflectivity, resistance, universal gas constant \\
\hline ROI & region of interest \\
\hline$\sigma$ & lateral tension \\
\hline SDCLM & spinning disc confocal laser microscopy \\
\hline SDS & sodium dodecyl sulfate \\
\hline SEM & scanning electron microscopy \\
\hline SICM & scanning ion conductance microscopy \\
\hline SNR & signal to noise ratio \\
\hline SPR & surface plasmon resonance \\
\hline STED & stimulated-emission-depletion \\
\hline SUV & small unilamellar vesicle \\
\hline$t$ & time \\
\hline$T$ & temperature \\
\hline TRIS & tris(hydroxymethyl)aminomethane \\
\hline UV/VIS & ultraviolet/visible \\
\hline 9 & wave vector \\
\hline QE & quantum efficiency \\
\hline
\end{tabular}




\section{A.7 Chemicals and consumables}

ammonia solution $(25 \%)$

Alexa Fluor 488 carboxylic acid TFP ester

argon

avidin

Atto fluorophores

BSA

Bodipy-TMR-PIP 2

cantilever MLCT

cap-biotin-DOPE

cholesterol

chloroform

DOPC

DOPE

disodium hydrogen phosphate

DMSO

DPhPC

ethanol, p.a.

ethanolamine hydrochloride

1-ethyl-3-(3-dimethylaminopropyl)

carbodiimide

glass slides D $263 \mathrm{M}$ Schott glass

$25 \mathrm{~mm} \times 75 \mathrm{~mm}$

glucose

glycerin

gold $(99.99 \%)$

HEPES

hydrochloric acid
VWR International, Darmstadt, GER

Invitrogen, Eugene, USA

Air Liquide Deutschland GmbH, Düsseldorf, GER

VWR International, Darmstadt, GER

Atto-tec GmbH, Siegen, GER

Carl Roth GmbH, Karlsruhe, GER

Echelon Biosciences, Salt Lake City, USA

Bruker AFM Probes, Calle Tecate, USA

Avanti Polar Lipids, Alabaster, USA

Sigma-Aldrich, Taufkirchen, GER

VWR International, Darmstadt, GER

Avanti Polar Lipids, Alabaster, USA

Avanti Polar Lipids, Alabaster, USA

Merck KGaA, Darmstadt, GER

Carl Roth GmbH, Karlsruhe, GER

Avanti Polar Lipids, Alabaster, USA

VWR International, Darmstadt, GER

Carl Roth GmbH, Karlsruhe, GER

Carl Roth GmbH, Karlsruhe, GER

ibidi GmbH, München, GER

Carl Roth GmbH, Karlsruhe, GER

Honeywell Riedel-de Haën AG,

Seelze, GER

Allgemeine Gold- und

Silberscheideanstalt, Pforzheim, GER

Carl Roth GmbH, Karlsruhe, GER

Merck, Darmstadt, GER 
hydrogen peroxide solution (30\%)

ITO slides

magnesium chloride hexahydrate

mercaptohexanol

methanol

MOPS

n-propanol

$N$-Hydroxysuccinimid

Parafilm $^{\circledR}$

POPC

POPE

$\mathrm{PIP}_{2}$

polycarbonate membranes

potassium chloride

potassium dihydrogen phosphate

potassium hydroxide

porous E800 substrates

porous Si substrates

porous $\mathrm{SiO}_{2}$ substrates

n-propanol

oxygen

silicon wafers

sodium chloride

sodium citrate

sodium dodecyl sulfate

SPR CMD500I sensorchip
Carl Roth GmbH, Karlsruhe, GER

Präzisions Glas \& Optik GmbH, Iserlohn, GER

Merck, Darmstadt, GER

Sigma-Aldrich, Taufkirchen, GER

Carl Roth GmbH, Karlsruhe, GER

Carl Roth GmbH, Karlsruhe, GER

Carl Roth GmbH, Karlsruhe, GER

Sigma Aldrich, Taufkirchen, GER

Pechiney Plastic Packaging, Chicago, USA

Avanti Polar Lipids, Alabaster, USA

Avanti Polar Lipids, Alabaster, USA

Avanti Polar Lipids, Alabaster, USA

Avestin, Ottawa, CAN

Merck, Darmstadt, GER

Merck, Darmstadt, GER

Merck, Darmstadt, GER

Nanospot GmbH, Münster, GER

provided by Dr. Siegfried Steltenkamp,

CEASAR, Bonn, GER

provided by Dr. Siegfried Steltenkamp,

CEASAR, Bonn, GER

Merck, Darmstadt, GER

Air Liquide Deutschland GmbH,

Düsseldorf, GER

Active Buisiness Company GmbH

Brunnthal, GER

Merck, Darmstadt, GER

Merck, Darmstadt, GER

AppliChem GmbH, Darmstadt, GER

XanTec bioanalytics $\mathrm{GmbH}$,

Düsseldorf, GER 
sucrose

Texas Red DHPE

TRIS

pyranine
Carl Roth GmbH, Karlsruhe, GER

Sigma-Aldrich, Taufkirchen, GER

Carl Roth GmbH, Karlsruhe, GER

Acros Organics, New Jersey, USA

\section{A.8 Devices}

\section{Atomic force microscopy}

Nanowizard 2

JPK Instruments AG, Berlin, GER

\section{Surface coating}

Zepto plasma cleaner

Diener Electronic, Ebbhausen, GER

Coating System MED020

Bal-Tec, Leica, Wetzlar, GER

Sputtercoater 108 auto

Cressington Scientific Instruments, Watford, UK

thickness Controller: MTM20

Cressington Scientific Instruments, Watford, UK

\section{Confocal laser scanning microscopy (CLSM)}

LSM 710 Examiner

objective:

WPlan APOChromat $63 \times / 1.0$

ENTH-Alexa 488

Texas Red DHPE
Carl Zeiss, Jena, GER

Carl Zeiss, Jena, GER

$$
\begin{aligned}
& \lambda_{\mathrm{ex}}=488 \mathrm{~nm}, \lambda_{\mathrm{em}}=499-567 \mathrm{~nm} \\
& \lambda_{\mathrm{ex}}=561 \mathrm{~nm}, \lambda_{\mathrm{em}}=569-699 \mathrm{~nm}
\end{aligned}
$$

\section{Spinning disc confocal laser microscopy (SDCLM)}

laser: iBeam Smart 405-S

laser: iBeam Smart 488-S

laser: Cobolt Jive ${ }^{\mathrm{TM}}$

AOTF: TF525-250-6-3-GH18A

stand: custom, based on ix73

spinning disc unit: Yokogawa CSU-X
Toptica Photonics AG, Gräfeling, GER

Toptica Photonics AG, Gräfeling, GER

Cobolt AB, Solna, Sweden

Gooch \& Housego PLC, Ilminster, UK Olympus, Tokyo, JAP

Rota Yokogawa GmbH \& Co. KG,

Wehr, GER 
piezo: P-721-CDQ

camera: iXON 897 Ultra

filter wheel: Rotr

filter: LP496 ET

filter: LP590 ET

filter: ET525/50

filter: ZET405/488/561/640

objective: CFI Plan 100XW 100×/1.1

Atto488-DPPE

Atto594-DPPE

ENTH-Atto594
Physik Instrumente, Karlsruhe, GER Andor Technology Ltd., Belfast, UK Andor Technology Ltd., Belfast, UK AHF Analysentechnik AG, Tübingen, GER AHF Analysentechnik AG, Tübingen, GER AHF Analysentechnik AG, Tübingen, GER AHF Analysentechnik AG, Tübingen, GER Nikon Düsseldorf, GER

$\lambda_{\text {ex }}=488 \mathrm{~nm}$, filter: LP496 ET,ET525/50

$\lambda_{\mathrm{ex}}=561 \mathrm{~nm}$, filter: LP590 ET

$\lambda_{\mathrm{ex}}=561 \mathrm{~nm}$, filter: LP590 ET

\title{
Scanning electron microscopy (SEM)
}

LEO SUPRA 35

Carl Zeiss, Jena, GER

\author{
Surface plasmon resonance spectroscopy (SPR) \\ spectrometer: SR700DC \\ Reichert, New York, USA \\ pump: SR7500 \\ Reichert, New York, USA \\ injection valve: SR8300 \\ Reichert, New York, USA \\ diverter: SR8600 \\ Reichert, New York, USA \\ degasser: DEG100 \\ XanTec bioanalytics $\mathrm{GmbH}$, \\ Düsseldorf, GER
}

Reflectometric interference spectroscopy (RIfS)

spectrometer: NanoCalc-2000, Ocean Optics, Dunedin, USA

SD2000

pump: Ismatec 795C

IDEX Health \& Science, Wertheim, GER

\section{Scanning ion conductance microscopy (SICM)}

stand: ix71

ICNano2000

amplifier: Axopatch 200B

oscilloscope TDS1001B
Olympus, Tokyo, JAP

Ionscope, Melbourn, UK

Axon Instruments, Union City, USA

Tektronix UK Ltd. Bracknell, UK 
piezo controller: E500K

puller: P1000

\section{Miscellaneous devices}

frequency generator: Agilent 3220A

NanoDrop 2000c

extruder

osmometer: Osmomat 030,

Osmomat 3000

pH-meter: Calimatic 766

ultrasonic bath: Sonorex RK 255 H
Physik Instrumente, Karlsruhe, GER

Sutter Instruments, Novato, USA

\section{A.9 Software}

ImageJ $1.45 \mathrm{~s}$

Origin $8.5 \mathrm{G}$

ZEN 2012

Matlab2014a

Gwyddion 2.34

Graphics Layout Engine

IQ 2.9

Spectra Suite

ICNano
Agilent Technologies, Santa Clara, USA

Thermo Scientific, Wilmington, USA

Avestin, Ottawa, CAN

Gonotec, Berlin, GER

Knick, Berlin, GER

Badelin, Berlin, GER http://rsbweb.nih.gov/ij

OriginLab Cooperation,

Northampton, USA

Carl Zeiss, Jena, GER

MathWorks, Natick, USA

http://gwyddion.net/

http://www.gle-graphics.org/

Andor Technology Ltd., Belfast, UK

Ocean Optics, Dunedin, USA

Ionscope, Melbourn, UK 



\section{Curriculum vitae}

\section{Personal data}

$\begin{array}{ll}\text { Name: } & \text { Martin Gleisner } \\ \text { date of birth: } & 12^{\text {th }} \text { December } 1987 \\ \text { place of birth: } & \text { Weimar } \\ \text { Citizenship: } & \text { German }\end{array}$

\section{Education}

2013-2016 Ph.D thesis at the Georg August University, Göttingen (Prof. Dr. Steinem). Title of thesis: Interaction of Epsin N-Terminal Homology domain (ENTH) with artificial lipid membranes as a function of lateral tension.

2010-2012 Graduate studies in chemistry at the Georg August University, Göttingen (Prof. Dr. Steinem). Master thesis: Untersuchung von Transportvorgängen an porenüberspannenden Membranen.

2011-2012 Erasmus stay at the University of Manchester, Manchester. Research thesis: The synthesis of a M1 muscarinic receptor agonist.

2007-2010 Undergraduate studies in chemistry at the Georg August University, Göttingen (Prof. Dr. Steinem). Bachelor thesis: Einfluss der molekularen Struktur des Rezeptorlipids $G_{3}$ auf die Shiga Toxin-Gb $b_{3}$ Wechselwirkung.

2006-2007 Civilian service at the Arbeiterwohlfahrt "Seniorenheim am Paradies", Weimar.

2004-2006 Secondary school 2 at the Friedrich Schiller Gymnasium, Weimar. 


\section{Scientific contributions}

\section{Peer-reviewed publications}

2014

Gleisner, M., Mey, I., Barbot, M., Dreker, C., Meinecke, M., \& Steinem, C. Driving a planar model system into the $3^{\text {rd }}$ dimension: Generation and control of curved pore-spanning membrane arrays, Soft matter 2014, 10(33), 6228-6236.

\section{Oral presentations}

2015 CRC803 autumn school, Altenau. How the ENTH domain alters the tension of lipid bilayers.

2014 Molecular Membrane Biophysics, Hünfeld 2014. Controlling curvature in pore-spanning membranes.

2014 CRC803 winter school, Volkenroda. Generation and Control of Curved pore-spanning Membrane Arrays.

\section{Selected poster presentations}

$201510^{\text {th }}$ European Biophysics Congress, Dresden. Impact of the ENTH domain on protruded pore-spanning membranes.

2014 Annual Meeting of the German Biophysical Society, Lübeck 2014. Protuded pore-spanning membranes.

$2013 \quad 9^{\text {th }}$ European Biophysics Congress, Lisbon. Curved pore-spanning membranes - Generation and application.

\section{Awards}

Poster prize at the 585 th WE-Heraeus-Seminar "Advanced Microscopy of Membrane Biophysics", Bad Honnef. 


\section{Danksagung}

Ich möchte mich ganz herzlich bei Prof. Dr. Claudia Steinem für die Betreuung der Doktorarbeit und die vielen konstruktiven wissenschaftlichen Gespräche während der gesamten Promotionszeit bedanken.

Prof. Dr. Michael Meinecke danke ich für die Übernahme des Zweitgutachtens sowie für die durchweg sehr gute Kooperation und die damit verbundenen Ideen.

Bei Prof. Dr. Sarah Köster und Prof. Dr. Jörg Enderlein möchte ich mich für die wertvollen Ratschläge in den Thesis Committees bedanken.

Prof. Dr. Andreas Janshoff danke ich für die Unterstützung, die vielen Diskussionen und Matlabskripte rund um die Auswertung der Messdaten.

Dr. Ingo Mey danke ich für den vielen fachlichen Austausch über mein Thema und die damit verbundenen Lösungsansätze. Auch unsere privaten Gespräche habe ich sehr genossen, erlaubten sie mir ständig neue Sichtweisen auf die offensichtlichen und weniger offensichtlichen Dinge des Lebens.

Mein Dank gilt außerdem Benjamin Kroppen für das unermüdliche Bereitstellen des Proteins und für die konstruktive Zusammenarbeit.

Bei meinem Masterstudent Jeremias und meinen beiden Bachelorstudenten Christian und Julian möchte ich mich für die engagierte und sorgfältige Bearbeitung ihrer Themen bedanken. Eure Beiträge haben dieser Arbeit wertvolle Aspekte hinzugefügt. 
Unseren technischen Angestellten Jutta Gerber-Nolte und Michaela Klingebiel danke ich ganz herzlich für die Unterstützung bei diversen Laborarbeiten und für das unermüdliche Messen von Osmolalitäten.

Vielen Dank auch an alle, welche meine Arbeit Korrektur gelesen haben und so wertvolle Ideen miteinfließen ließen.

Ich danke dem gesamten Arbeitskreis für die schöne Zeit und den kollegialen Zusammenhalt. Ich werde unsere gemeinsamen Mess-, Grill- und Frühstückszeiten, sowie die stets sachlichen und politisch einwandfreien Diskussionen im Kaffeeraum sehr vermissen.

Weiterhin möchte ich mich bei meiner Familie für unentwegte Unterstützung mit Rat und Tat bedanken. Wann immer ich Probleme hatte, konnte ich mich an euch wenden. 